\title{
Banking market Integration in the SADC countries: evidence from interest rate analyses
}

Citation for published version (APA):

Aziakpono, M., Kleimeier, S., \& Sander, H. (2007). Banking market Integration in the SADC countries:

evidence from interest rate analyses. METEOR, Maastricht University School of Business and Economics. METEOR Research Memorandum No. 047 https://doi.org/10.26481/umamet.2007047

Document status and date:

Published: 01/01/2007

DOI:

10.26481/umamet.2007047

Document Version:

Publisher's PDF, also known as Version of record

\section{Please check the document version of this publication:}

- A submitted manuscript is the version of the article upon submission and before peer-review. There can be important differences between the submitted version and the official published version of record.

People interested in the research are advised to contact the author for the final version of the publication, or visit the DOI to the publisher's website.

- The final author version and the galley proof are versions of the publication after peer review.

- The final published version features the final layout of the paper including the volume, issue and page numbers.

Link to publication

\footnotetext{
General rights rights.

- You may freely distribute the URL identifying the publication in the public portal. please follow below link for the End User Agreement:

www.umlib.nl/taverne-license

Take down policy

If you believe that this document breaches copyright please contact us at:

repository@maastrichtuniversity.nl

providing details and we will investigate your claim.
}

Copyright and moral rights for the publications made accessible in the public portal are retained by the authors and/or other copyright owners and it is a condition of accessing publications that users recognise and abide by the legal requirements associated with these

- Users may download and print one copy of any publication from the public portal for the purpose of private study or research.

- You may not further distribute the material or use it for any profit-making activity or commercial gain

If the publication is distributed under the terms of Article $25 \mathrm{fa}$ of the Dutch Copyright Act, indicated by the "Taverne" license above, 
Meshach Aziakpono, Stefanie Kleimeier, Harald Sander

Banking Market Integration in the SADC

Countries: Evidence from Interest Rate Analyses

$\mathrm{RM} / 07 / 047$

JEL code: E42, E43, E52, E58, F36

\section{METE@R}

Maastricht research school of Economics of TEchnology and ORganizations

Universiteit Maastricht

Faculty of Economics and Business Administration

P.O. Box 616

NL - 6200 MD Maastricht

phone : ++31433883830

fax : ++31433884873 
METEOR Version: November 5, 2007

\title{
BANKING MARKET INTEGRATION IN THE SADC COUNTRIES: EVIDENCE FROM INTEREST RATE ANALYSES ${ }^{1}$
}

\author{
by \\ Meshach Aziakpono a , Stefanie Kleimeier ${ }^{\mathrm{b}, \mathrm{d}^{*}}$ and Harald Sander ${ }^{\mathrm{c}, \mathrm{d}}$ \\ a Department of Economics and Economic History, Rhodes University, P.O. Box 94, \\ Grahamstown 6140, South Africa. \\ ${ }^{\mathrm{b}}$ Limburg Institute of Financial Economics, FdEWB, Maastricht University, Tongersestraat \\ 53, 6211 LM Maastricht, The Netherlands. \\ ${ }^{\mathrm{c}}$ Faculty of Economics and Business Administration, University of Applied Sciences Cologne, \\ Claudiusstr.1, 50678 Köln, Germany. \\ ${ }^{\mathrm{d}}$ METEOR, Maastricht University, Tongersestraat 53, 6211 LM Maastricht, The Netherlands.
}

\begin{abstract}
This paper investigates the state, development and drivers of banking market integration in the member countries of the Southern African Development Community (SADC) by employing interest rate data. We first conduct a principal component analysis and find evidence for both increasing monetary integration and banking integration in loan and deposit markets. These integration processes are not developing uniformly and we can identify a convergence club. As banking market integration can be a genuine process or simply be driven by monetary integration, we also investigate the interest rate pass through from national and South African Central bank interest rates onto national retail rates. With respect to the convergence club we find both, genuine and monetary-integration driven processes though the latter dominate. We conclude that a selective expansion of the Common Monetary Area is possible but needs to be complement by efficient financial development policies.
\end{abstract}

JEL Classification Numbers: E42; E43; E52; E58; F36.

Keywords: Banking Market Integration, Monetary Policy, Southern Africa, Common Monetary Area, SACU, SADC, Interest Rate Pass-Through, Principle Components.

* Corresponding author. Tel: +31-43-3883733; fax: +31-43-3884875; E-mail address: s.kleimeier@finance.unimaas.nl (Stefanie Kleimeier). 


\section{Introduction}

Economic and financial integration ranks high on the policy makers' agenda in Africa. Among the various regional integration initiatives ${ }^{1}$, the Southern African Development Community (SADC) has recently become a focal point of interest. SADC has traditionally focused on trade and structural policies but has recently announced to aim at monetary unification by the year 2016 (Rossouw, 2006). It comprises such diverse countries as Angola, Botswana, Democratic Republic of Congo (DRC), Lesotho, Madagascar, Malawi, Mauritius, Mozambique, Namibia, Seychelles, South Africa, Swaziland, Tanzania, Zambia and Zimbabwe of which only four, namely South Africa, Lesotho, Swaziland, and Namibia already form a Common Monetary Area (CMA). Most studies on the viability of this vision concentrate either on optimum currency area (OCA) considerations or macro-economic (nominal) convergence (see Jenkins and Thomas, 1998; McCarthy, 2002; Kabundi and Loots, 2007; Rossouw 2006). In contrast and as a complement to these exercises our study concentrates on the state and potential development of monetary and financial integration across SADC countries. In choosing this point of view we also implicitly acknowledge the new strand of literature on endogenous OCAs which start with Rose (2001) and hypothesize a potentially large positive effect of a common currency on trade. In this paper we investigate rigorously the current state and underlying causes of banking market integration among SADC countries by bringing together two strands of the empirical financial integration literature, principal component analysis (PCA) and interest rate pass-through (PT) analysis, with a view on deriving policy conclusion on the readiness of countries for joining a monetary area or union based on monetary and financial integration criteria.

\footnotetext{
${ }^{1}$ Most of the regional alliances are focusing on common economic and trade policies, such as the Common Market for Eastern and Southern Africa (COMESA) and Economic Community of West African States (ECOWAS), whereas the 14 member countries of the African Financial Community (CFA) form two monetary unions, the West African Economic and Monetary Union (WAEMU) and the Central African Economics and Monetary Community (CEMAC) with a single central bank in each union and a single currency or peg to the French franc/euro, respectively.
} 
Financial integration can be measured in various ways. A prominent classification distinguishes quantity-based, price-based and new-based measures (Baele et al., 2004). Quantity-based measures focus on cross-border transactions, such as cross-border loans and deposit taking or cross-border mergers and acquistions (M\&As). As these data are either notoriously unreliable or unavailable for SADC countries, price-based measures, in our case interest rates, are preferable. Price-based measures of integration regularly use the law of one price (LOOP) as the point of reference (Adam et al., 2002). In the case of financial integration, the relevant parity conditions are interest rate parity conditions. While this is acceptable for analyzing homogeneous assets, it is particularly questionable in the case of retail banking. In the European context (Kleimeier and Sander, 2000) therefore advocate to investigate comovements rather than convergence of retail interest rates. In a structurally stable environment this can be done by means of co-integration analysis. During a nominal convergence process when inflation rates are aligning in anticipation of a monetary union, co-integration analysis is not appropriate as a long-run relationship may not exist during the convergence process. Here it is more appropriate to analyze co-movements of interest rates by PCA that can also identify groups of countries for which interest rates move in the same direction. In order to investigate the monetary and banking market integration in SADC, we applying PCA to central bank (monetary policy) interest rates, deposit and loan interest rates, respectively. The European experience has however made clear that in the presence of integrated money markets or monetary policy rates, co-movements of retail interest rates can easily be misread as sign of an integrated banking market (Kleimeier and Sander, 2007). If central bank rates move together and monetary policy rates are transmitted to retail rates in a uniform way in the various countries, then the banking market appears integrated. Therefore an analysis of the national interest rate PT should complement PCA. This news-based measure thus reports how similar the banking markets in the investigated countries respond to monetary policy news. 
In this study we contribute to the literature by focusing on the state of and potential for financial integration in SADC with special reference to banking market integration. The existing empirical evidence focuses either on measuring integration or on measuring the PT, but no study has brought both elements together. This is where our contribution lies. First, we investigate monetary and banking market integration using PCA. By applying PCA to different time periods, i.e. rolling time windows, we can document the progress and development of financial integration over time. Second, we look at the driving forces of banking market integration and single out the role of an efficient PT of national monetary policy onto bank interest rates or - if applicable - the role of a common or dominant monetary policy. The latter will be explored by investigated directly the PT from South African to domestic banking rates. Our findings indicate that the degree of financial integration is increasing in some but not all countries. While banking market integration is predominantly driven by monetary integration, a genuine banking market integration process is also observable in some countries. However, even this integration process is concentrated on a "convergence club" with CMA in its centre. Our analysis indicates that a few non-CMA SADC countries may become potential candidates for a CMA enlargement, while many others are still far away from being judged as candidates from a pure financial integration point of view.

The plan of the paper is as follows: Section 2 reviews the state of banking market integration in Southern Africa as it is documented in the literature. Section 3 describes data and methodologies used. Section 4 reports the results and Section 5 concludes.

\section{Financial Development and Integration in SADC: Stylized facts and literature review}

SADC comprises 15 countries which differ widely in terms of development, macroeconomic stability, monetary and financial development and real and financial integration. Of these countries four are currently member of the CMA and are thus operating under a fixed exchange rate systems with the South African rand as anchor currency. 
Botswana, that together with the four CMA countries forms the Southern African Customs Union (SACU), has an independent exchange rate system but is indirectly linked to the rand through a currency basket in which the rand accounts for 60 to $70 \%$. The other 10 countries (OTHER) are following largely differing exchange rate regimes. ${ }^{2}$ Table 1 give an overview on the development of some important real and financial development indicators of the individual SADC countries as well as the GDP-weighted averages for all SADC countries and the subgroups CMA, SACU and OTHER. As expected, CMA shows the highest degree of macroeconomic convergence, especially with respect to inflation. Botswana's characteristics are in a similar range but the diversity is much higher in the OTHER countries. Nevertheless, also this group has nominally converged as a dis-inflation policy was successful in many, though not all countries. Comparing SADC's achievements with respect to the Maastricht criteria for convergence and thus membership in the European Monetary Union (EMU), Rossouw (2006) however argues that "the challenges facing a SADC monetary union would not be insurmountable if the convergence criteria are viewed as permanent goals, rather than preconditions.” In contrast, Buigut and Valev (2006) are less optimistic. They point to South Africa's resistance to a CMA expansion and conclude that the CMA could easiest be expanded by Botswana, Mozambique and Zambia.

[Insert Table 1 about here]

Looking at financial development reflected by the spread of lending over deposit rates as an indicator of banking market efficiency and financial deepening indicators such as credit to GDP and deposits to GDP clearly shows the enormous diversity in financial development as well as the well-documented financial underdevelopment in many SADC countries. Clearly,

\footnotetext{
${ }^{2}$ For a summary of exchange rate systems, exchange control restrictions on current and capital account transactions in the SADC countries, see Table A-1 in Appendix A.
} 
South Africa and Namibia have the most developed banking markets along with non-CMA member Mauritius. Moreover these measures improve slightly over time. Financial development is also taking place in the remaining countries but is still on a very low level. Not much progress is being made in the latter group with respect to the spreads. To the contrary, this efficiency measure shows improvement in only very few countries and is still particularly high in the OTHER countries.

Given the strong differences in financial intermediation, bank interest rates and spreads several questions arise when capital movements are not (fully) resticted ${ }^{3}$ : Are arbitrage processes taking place across SADC banking markets? To what extent are these processes already effective in integrating SADC banking markets? If retail banking rate are in fact comoving, to what extent is this being mediated through co-movements of monetary policydetermined interest rates?

The existing empirical studies on interest rates in Southern Africa focus either on measuring integration by interest rate co-movement (Aziakpono, 2006a and 2006b; Nielsen et al., 2005) or on measuring the PT (Sander and Kleimeier, 2006). Aziakpono (2006b) and Nielsen et al. (2005) investigate the behavior of national interest rates by using the interest rate parity as an indicator of integration. Aziakpono's (2006b) findings reveal a high level of dependence of the other SACU countries' financial systems on South Africa's and he thus concludes that a monetary unification with a single central bank (South African Reserve Bank) and monetary policy for the union is feasible. However, the success of such a monetary union will critically depend on the efficiency gains of the domestic financial system. Such gains are country-specific. For countries such as Botswana, Lesotho and Swaziland with relatively weak domestic monetary policy transmission, a significant improvement in their monetary transmission can be expected. However, in Namibia, where the domestic policy seems to be more effective, a single central bank may actually lower the speed of the monetary transmission 
process. These findings are in line with Nielsen et al. (2005) who use the concept of the uncovered interest rate parity to assess the level of financial integration within the CMA plus Botswana, Zambia and Zimbabwe. Using both rolling unit root tests and a moving regression that enable them to examine the degree of financial integration over time, they find that Lesotho, Namibia and Swaziland are well financially integrated with South African market while the other countries are not. This has led to the formulation of the South African dominance hypothesis (SADH) whose proponents of the SADH consider South Africa to be of particular importance for two reasons: First, South Africa coordinates the Finance and Investment Sector of SADC that is responsible for coordinating fiscal and monetary policy. South Africa also chairs the Committee of Central Bank Governors (CCBG) since its establishment in 1995 to promote close cooperation among central banks in the area of monetary policy, monetary policy instruments, bank supervision and financial institutions in the SADC region and has since inception been chaired by South Africa and has its secretariat in South Africa. Second, South Africa has the largest and most developed financial system in the region. Direct evidence for the SADH is provided by Aziakpono (2006a) who examines the extent of interest rate PT to measure the degree of financial integration amongst SACU countries. His findings support the SADH and reveal a hierarchy of financial integration with South Africa ranging from highly-integrated Namibia and Swaziland to less-integrated Lesotho and least-integrated Botswana. The results further suggest that policy convergence, rather than market convergence, as the main driver of integration. In a related study, Sander and Kleimeier (2006) find a rather homogeneous PT process for lending rates and a less homogeneous one for deposit rates. Again South African interest rates play an important role in the determination of national discount rates, T-bill rates as well as commercial bank interest rates. They thus confirm the finding of Aziakpono (2006a) and the validity of the SADH. Existing studies focus on the CMA or SACU countries, with exception of Nielsen et al. (2005) who included Zambia

\footnotetext{
${ }^{3}$ For country details on restrictions on capital account transaction see Table A-1 in Appendix A.
} 
and Zimbabwe. None of the studies have investigated the extent of integration across the entire set of SADC countries. As such, our study is also the first to provide directly comparable evidence for all SADC countries.

\section{Data and Methodology}

\subsection{Data selection and source}

We investigate the integration of central bank and retail banking markets by analyzing the interest rates that are charged in these markets. We therefore collect monthly interest rate series for all 15 SADC countries from the International Financial Statistics (IFS). We use discount rates provided in IFS series 60 as central bank rates, prime lending rates of series 60P as lending rates, and series 60L as deposit rates. In general, our sample extends from January 1990 to December 2005 but several series have missing values. For details see Table A-2 in Appendix A.

As the economic environment in Southern Africa is rather volatile, we have to define an appropriate time horizon for our analysis. On the one hand, a shorter sample period will better reflect changes that occur in the banking markets. On the other hand, a longer sample period will provide statistically sounder results. To balance these two aspects, we opt for rolling sample periods of five years (60 observations) each. We can thus analyze 12 samples from January 1990 to December 1994, January 1991 to December 1995, and so on until January 2001 to December 2005. While sufficiently long for statistical analysis, the rolling nature of the sample periods should reveal any changes in integration over time.

Figure 1 gives a first impression of the development of interest rate dispersion over time in four regions: (1) CMA which consists of South Africa, Lesotho, Namibia and Swaziland; (2) SACU which consists of all CMA countries plus Botswana; (3) SADC which includes all five SACU countries plus Angola, DRC, Madagascar, Malawi, Mauritius, Mozambique, Seychelles, Tanzania, Zambia and Zimbabwe; and (4) our final group of OTHER countries which includes 
all SADC countries that are not SACU members. The coefficient of variation - defined as the standard deviation of all regional interest rates divided by their mean - reveals that interest rates are more homogenous in CMA and SACU. Due to the exchange rate union among CMA countries and - to lesser extent - to Botswana, this result is not surprising. Taking the region as a whole, the degree of dispersion starts to increase in late 2003. While this increase occurrs simultaneously for retail rates and central bank rates in the non-SACU countries, lending rate dispersion does not increase in the CMA.

\section{[Insert Figure 1 about here]}

\subsection{Methodology}

The developments of interest rate dispersion in SADC countries suggest two avenues for subsequent investigation. First, we will scrutinize the development and extent of integration of both central bank and retail interest rates. As Figure 1 clearly illustrates, the integration (or disintegration) process of retail banking can be dominated by monetary integration as it could be inferred from the recent developments of the non-SACU OTHER countries. Looking however at CMA and - to a much lesser extent at SACU - banking markets may also lead the process. Thus, we will pay attention to the issue whether financial integration is driven by banking market or by monetary integration. Secondly, if monetary integration is the driver of banking market integration, it requires that in the process of monetary integration the PT from central bank rates to retail interest rates will not become more heterogeneous. If the PT remains unchanged, the developments in banking markets are simply following monetary integration. If the PT becomes more homogeneous under increasing monetary integration - or more heterogeneous under monetary disintegration - this would reflect more banking market integration. The latter can happen particularly in times where banking interest rates follow the 
national central bank rates to a lesser degree than before and instead orient themselves more to central bank rates of a dominant foreign monetary policy.

We use two methodological approaches, PCA and PT analysis, to examine the extent of banking market integration among SADC countries. As the more detailed presentation of these methodologies in the next sections will show, the PCA documents the progress and development of integration over time while the PT analysis reveals the driving forces of banking market integration, particularly the role of an efficient PT of national monetary policy onto bank interest rates and the role of a common or dominant monetary policy. With respect to SADH this means that when the South African Reserve Bank has a direct and unidirectional impact on monetary policy rates in other country and an efficient PT exists in South Africa and the respective other country, then the banking markets also appear to be integrated by means of monetary policy convergence rather than a genuine banking market convergence which could also be dominated by South African markets. A third version of the SADH could envisage a direct impact of South African monetary policy rates on bank interest rates in third countries even without monetary integration when banks orient their pricing policy to South African policy rates.

\subsubsection{Principal component analysis}

Analyses of financial integration based on interest rate levels follow one of two approaches. The first was introduced by Cooper (1971) who examines the degree of divergence of interest rates across countries based on their standard deviation. According to this approach, the lower the standard deviation of yields on similar assets the higher the degree of financial integration; whereas if the standard deviation is high, then the level of integration is low (Nellis, 1982). The second approach popularized by Logue et al. (1976) focuses on the covariability of interest rates and does not require similarity of interest rate levels. According to this approach, the degree of financial integration will be high if the covariability of interest 
rates and their changes is high despite the fact that interest rate levels may differ substantially. As Figure 1 shows, this is the case for SADC countries and we thus follow this second approach and assess the covariability of interest rates using PCA. ${ }^{4}$

The aim of PCA is to obtain a small number of uncorrelated factors, called principal components (PCs), that best account for the correlation among the interest rates in the different countries. Let $\mathrm{X}$ represents a vector of interest rates in the CMA countries. PCA detects banking market integration by converting a matrix of these rates into a linear combination of unobserved principal components, which explain the complete variance of these interest rates. The PC problem can thus be expressed as

$$
\text { (1) } P=A X
$$

where $\mathrm{P}$ is a vector of orthogonal factors or PCs which are a linear combination of the original interest rate series $\mathrm{X}$ and $\mathrm{A}$ is a matrix of coefficients, called factor loadings, with each coefficient representing the weight of the corresponding original variable in the relevant PC. The number of PCs is equal to the number of the original variables. However, the PCs are orthogonal, i.e. independent of each other. The PCA derives the PCs in such a way that they explain the variations in the set of original variables in a descending order. Thus, the first PC accounts for the main part of the variation in X; the second PC will explain the main part of the remaining variations in $\mathrm{X}$ after the effects of the first PC has been removed. The transformation of variables can be accomplished by using either the correlation matrix or the covariance matrix. Since it is mean-centered, the correlation matrix is particularly useful when the variables differ in scale (Jolliffe, 2002). We use Southern African interest rates in levels which have varying scales because of differences in national inflation and exchange rates. Hence, in this paper we employ the transformation based on the correlation matrix. ${ }^{5,6}$ If banking markets

\footnotetext{
${ }^{4}$ Next to the contribution to the understanding of Southern African banking markets, our study is thus also contributing to the growing literature which assesses regional integration based on PCA (Perignon et al., 2007; Becker and Hall, 2007; Gilmore et al., 2007; Figueira et al., 2005; Siliverstovs et al., 2005; Fernandez-Izquierdo and Lafuente, 2004; Nellis, 1982).

${ }^{5}$ For a detailed description see Jolliffe (2002: 21-22).
} 
are integrated, the correlation structure of the interest rates would be best described by one common factor explaining their long run behavior. But if banking markets are not integrated, there will be more than one PC needed to explain the interrelations between bank interest rates.

In order to determine the explanatory power of each PC, two measures are conventionally used: the eigenvalue and the cumulative $\mathrm{R}^{2}$ of the PC. To determine the significance of a PC we apply the Kaiser rule of eigenvalue greater or equal to 1 (Kaiser, 1960). ${ }^{7}$ Next to the relative contribution of each country's interest rate to the significant PCs, we used the factor loadings of the PCs. Note that the signs of the loadings are completely arbitrary (Joliffe, 2002:67). However, the pattern of variation of the signs from one factor loading to the other can be used to determine the co-movement of the variables. In the context of our analysis, a largely random factor loading (with variable signs and sizes) implies that the interest rates move largely independently of each other, hence indicating low integration. The more systematic (same sign and size) the loadings, the greater the degree of integration (Becker and Hall, 2007). In addition, the pattern exhibited by a group of countries can be used to determine a convergence group, i.e. countries that move in the same direction (having the same sign in the same PC). Even though PCA is a static analysis, in this paper, following Gilmore et al. (2007), we tailored it to a dynamic analysis to examine the progress and development of banking market integration over time. This is accomplished by estimating the models for rolling time windows of five years each and then graphically plotting the eigenvalues.

\subsubsection{Empirical pass-through analysis}

The PT methodology is closely based on Sander and Kleimeier (2004, 2006) who model the PT as the following vector autoregressive (VAR) process:

\footnotetext{
${ }^{6}$ Note also that the PCA makes no assumptions about the underlying properties of the data series $\mathrm{X}$. Thus there is no need for instance to determine the stationarity properties of each series.

${ }^{7}$ For a discussion of weaknesses and alternative rules see Jolliffe (2002:112-131). For applications see for instance Nellis (1982), Figueira et al. (2005) and Meric et al. (2007).
} 


$$
\text { (2) } R R_{t}=\beta_{0}+\sum_{i=1}^{k^{*}} \beta_{R R, i} R R_{t-i}+\beta_{1} C B R_{t}+\sum_{i=1}^{n^{*}} \beta_{C B R, i} C B R_{t-i}+\varepsilon_{t}
$$

where $\mathrm{CBR}_{\mathrm{t}}$ is the central bank rate and $\mathrm{RR}_{\mathrm{t}}$ represents the commercial banks' retail rate which can be either a lending rate (LR) or a deposit rate (DR). The optimal lag length is indicated by $\mathrm{k}^{*}$ and $\mathrm{n}$, respectively, and determined according to the AIC criterion over all combinations of lags up to a lag length of 4. Based on this representation of the PT process, the responsiveness of RRs to CBRs can be measured over various time horizons. The impact multiplier, as given by the coefficient $\beta_{1}$, measures the immediate adjustment within the current month. Here a value of $\beta_{1}<1$ indicates sluggish adjustment, also known as interest rate stickiness. At the other end of the time horizon, the long-term multiplier can be calculated from (2) as

$$
\text { (3) } \theta=\frac{\beta_{1}+\sum_{i=1}^{n^{*}} \beta_{C B R, i}}{1-\sum_{i=1}^{k^{*}} \beta_{R R, i}}
$$

In the long run equation (2) therefore has the form of

$$
\text { (4) } R R_{t}=\theta_{0}+\theta C B R_{t}+u_{t}
$$

If $\theta=1$, we speak of a full PT in the long run. Switching costs, information asymmetries, not fully elastic credit demand functions, imperfect competition and other market imperfections can cause an imperfect PT, in which case we will find $\theta<1 . \theta>1$ can represent times when banks do not ration credit supply but increase lending rates to compensate for higher risks.

As is common for interest rate time series, the CBRs and RRs used in this study exhibit an I(1) property - that is, they are non-stationary series that do not return to any mean value and that have with time increasing variances. ${ }^{8}$ In this case, PT models like equation (2) are regularly estimated in first differences to avoid spurious regression problems:

\footnotetext{
${ }^{8}$ In contrast, $\mathrm{I}(0)$ or stationary series have a limited variance and fluctuate around their mean. By differentiating an I(1) series, a I(0) series is obtained. We employ various tests to establish whether or not the interest rate series used in this study are non-stationary. For each of the rolling sample periods, we conduct ADF and DG-GLS unit root tests. For the full period, we additionally estimate mean-shift, trend-shift and recursive unit root tests which
} 


$$
\text { (5) } \Delta R R_{t}=\beta_{0}+\sum_{i=1}^{k^{*}} \beta_{R R, i} \Delta R R_{t-i}+\beta_{1} \Delta C B R_{t}+\sum_{i=1}^{n^{*}} \beta_{C B R, i} \Delta C B R_{t-i}+\varepsilon_{t}
$$

It should be noted that by doing so, important information about the long-run relationship is lost. Equation (2) can, however, be estimated directly and will contain the long-run information if both interest rates are cointegrated in the sense of Engle and Granger (1987). ${ }^{9}$ The deviation from the long-run equilibrium will then be measured by the estimated error $\mathrm{u}_{\mathrm{t}}$ from equation (4) and included in the PT model as a lagged error correction term (ECT). Thus, a proper PT measurement should be based on an error correction model (ECM) that explicitly incorporates the long-run relationship between RRs and CBRs provided the series are cointegrated:

(6) $\Delta R R_{t}=\beta_{0}+\sum_{i=1}^{k^{*}} \beta_{R R, i} \Delta R R_{t-i}+\beta_{1} \Delta C B R_{t}+\sum_{i=1}^{n^{*}} \beta_{C B R, i} \Delta C B R_{t-i}+\beta_{E C T} E C T_{t-1}+\varepsilon_{t}$

In order to better understand what determines the degree of integration among the different SADC countries, we investigate not only the effects of domestic monetary policy but also the impact of the South African monetary policy on national retail banking rates. The reason for this is self-evident for countries that belong to a currency union. In CMA, for example, due to the central role of the South African rand, the monetary policy of the South African central bank is leading and the other central banks have to mimic its policy in order to sustain the currency union and the conditions of perfect capital mobility. Consequently, commercial banks adjust their lending and deposit rates to a common monetary policy rate and may even adjust more directly to changes of the South African than to national policy rate.

are valid even in the presence of a structural break (see Banerjee, Lumsdaine, and Stock, 1992). Results can be found in Tables B-4 to B-6 in Appendix B. Our tests indicate that the series are I(1) but there are a few exceptions. As we however do not encounter cases where both the CBR and the RR are I(0), we always estimate the PT in first differences.

${ }^{9}$ We employ two types of tests in order to determine whether on not CBR and RR are cointegrated. First, we estimate the usual Durbin-Watson (DW), Dickey-Fuller (DF) and augmented Dickey-Fuller (ADF) tests. Cointegration is considered to exist if at least two test statistics are significant at $10 \%$ level or 1 test statistic at $5 \%$ level. Second, we follow Kremers, Ericsson and Dolado (1992) and consider cointegration to exist when the coefficient of the lagged error correction term $\left(\mathrm{ECT}_{\mathrm{t}-1}\right)$ is significant at the $5 \%$ level in equation (6). If one of both of these test procedures indicates cointegration, we estimate the PT as (6). Only if both of these test procedures reject cointegration is the PT estimated as (5). To avoid switching too frequently between these two PT models, i.e. for our overlapping sample periods, exceptions are made for single periods. Details can be found in Tables B-7 and B-8 in Appendix B. 
Beyond more or less fixed pegs to the rand, a significant response of retail rates to South African central banks rates is of course much less likely. Imagine, however, that banking markets are fully integrated in the absence of monetary integration while capital mobility is given only in retail banking. Consider under such conditions an increase in the South African central bank rate that also increases deposit rates. With an integrated banking market this would lead to cross-border arbitrage and eventually to higher deposit rates in the other countries. Hence, the existence of a PT from the South African policy rate to other countries' national banking market rates can be interpreted as evidence for banking market integration when no monetary integration is found. Thus, the PT analysis is an important complement to the PCA.

\section{$\underline{4 . \text { Results }}$}

\subsection{Banking market integration: Evidence from principal component analyses}

Tables 2, 3 and 4 and Figures 2 and 3 summarize the PCA results. ${ }^{10}$ Table 2 reports the number of factors that explain the joint variation of the national interest rates based on statistically significant PCs using the Kaiser rule of eigenvalues greater than one. Table 3 reports the cumulative $\mathrm{R}^{2}$ of the first PC, while Figures 2 and 3 plot the eigenvalues of the PCs and the factor loadings of the first PC, respectively. The results are reported by regional group as well as by interest rate. Lastly, Table 4 reports the results of the convergence group analysis based on the factor loadings of significant PCs for the period 2000 to 2005 when there was greater evidence of banking integration in the SADC region. Overall, the results confirm our expectation that CMA banking markets are most integrated followed by the SACU countries. With the exception of deposit markets, the OTHER countries are more integrated than SADC as a whole. There is also some evidence to suggest that the level of integration increases over time for each region and interest rate.

\footnotetext{
${ }^{10}$ Detailed results of the PCA can be found in Tables B-1 to B-3 in Appendix B.
} 
[Insert Tables 2 and 3 about here]

\subsection{1. $C M A$}

In the CMA with the exception of 1996-2000 rolling period for the central bank rate, in all the rolling windows there is only one significant PC. The cumulative $\mathrm{R}^{2}$ reported in Table 3 also confirm this. In all the cases, the first PC has a high explanatory power, explaining on average $82 \%, 87 \%$ and $86 \%$ of the variations in central bank, deposit and lending rates, respectively. Both retail rates exhibit stronger evidence of convergence and integration than the central bank rate. As evident from the graphical plot of the eigenvalues of the first PC in Figure 2 and the $\mathrm{R}^{2}$ in Table 3, the level of banking market integration among the CMA countries is relatively stable over time, only the $\mathrm{R}^{2}$ show some improvement except for the second half of the 1990s (1995-1999 and 1996-2000 rolling periods). The possible reasons for the slight drop in the degree of banking market integration during the $2^{\text {nd }}$ half of the 1990 s become apparent if one examines the factor loading for the CMA (see Figure 3). The factor loading of the first PC show that all countries move in the same direction as can be expected due to the CMA agreement. However, the coefficients for Lesotho are lower, implying that this country weighs less in the integration of the region. This effect is particularly manifest in the central bank rate, which in addition to being significantly lower, actually moves in the opposite direction during 1996-2000 rolling period. However, the trend changes with the 1997-2001 rolling period. The events in Lesotho lend support to the results. From the mid-1990s, the financial system in Lesotho experienced considerable distress which was mainly due to poor management, irresponsible lending and political interference in banking operations. This culminated in the liquidation of the Lesotho Agricultural Development Bank (the country’s second largest bank) in 2000 and the Lesotho Bank (the country’s largest bank) in 2001, despite several rescue attempts by the government. In August and September 1998, Lesotho was furthermore engulfed in a political crisis that led to destruction of property, heavy stock looting and burning 
of several companies. The uncertainty created by the crisis resulted in an additional decline in domestic banking activities (Aziakpono, 2004).

[Insert Figures 2 and 3 about here]

\subsection{2. $S A C U$}

As expected, the banking markets in the SACU countries are also highly integrated though to a lesser extent than the CMA countries. In most rolling periods, one significant PC is found. The explanatory powers of these first PCs are quite high, averaging $73.7 \%, 74.8 \%$ and 75.3\% for central bank, deposit and lending rates, respectively. Again, the retail bank rates show stronger evidence of convergence. The level of banking market integration however varies slightly over time. As for the CMA countries, SACU's banking markets experience the least integration during the second half of the 1990s, especially during 1995-1999. During 1997-2001 and 1998-2002, banking markets (especially the retail markets) experience an unprecedented level of integration but dropp again during 1999-2003, and thereafter begin to rise.

An examination of the coefficients of the first PC reveals the relative contributions of each country's market to the observed integration patterns. As can be observed in Figure 3, the pattern of movement of the CMA countries within SACU remains the same as observed when analyzing the CMA region alone. However, Botswana moves in a consistent manner in all the periods and independently of the rest of the SACU countries for most of the rolling periods and irrespective of the interest rates used. It is striking that for all the instances where the markets moved in harmony (2000-2004 and 2001-2005 for all interest rates and 1994-1998 for lending rates), the CMA countries converge towards Botswana and not the opposite. It is not quite evident what is responsible for the pattern during the 1994-1998 rolling period. One possible explanation might be the change in the political dispensation in South Africa in 1994 resulting 
in a friendlier relationship with Botswana, which hitherto maintained a strong opposition to South Africa. The concomitant relaxation of the political tension between the two countries might have manifested itself in the synchronization of their banking credit markets.

The pattern since 2000 can be explained by two possible factors. First, in February 1999 Botswana adopted a more liberal foreign exchange policy by abolishing the exchange control and adopting full capital account convertibility (see Table A-1 in Appendix A for a summary of the prevailing exchange rate systems). This policy action which signaled a move towards openness of the financial system might have contributed to the synchronization of interest rates. However, given the fact that the factor loadings for Botswana for all the interest rates maintain a consistent pattern over time, the change in policy stance in 1999 may not have been the major driver of the synchronization of interest rates with those of the CMA countries. Hence, in our view, a second factor may be the major driver: In 2000, South Africa adopted an inflation targeting monetary policy framework which resulted in a drop in both inflation rate and market interest rates (and of course the other CMA countries followed). Importantly, this brought stability to the market interest rates relative to the past, resulting in rates which were more in harmony with Botswana’s stable and lower interest rates.

\subsubsection{OTHER and SADC}

The none-SACU members of the SADC constitute the OTHER region. As expected the level of banking market integration is relatively low compared to SACU. However, unlike CMA and SACU where the retail bank rates lead the integration process, in OTHER countries the central bank rates lead the integration process followed by lending rates. With an average of $55.4 \%$, $45.4 \%$ and $51.7 \%$ for central bank, deposit and lending rates, respectively, the explanatory power of the first PC is low. Likewise, there are mostly two or more significant PCs. It is also evident that the degree of banking market integration increases during the last two rolling periods for all interest rates. The factor loadings are very random except in the last 
two rolling periods when they tend to exhibit a more systematic pattern. Finally, the results for the entire SADC region are quite similar to OTHER. It is also evident that in recent years, particularly since 2000, the degree of banking market integration increases.

\subsubsection{Convergence Club in the SADC region: 2000 - 2005}

We subject the entire SADC region to further analysis focusing on the period between 2000 and 2005 where there is evidence of growing integration. Our aim is to sort the countries into groups that are becoming integrated based on the similarities of their movements. Since our earlier analyses have shown that the CMA countries are highly integrated we will pay attention to those countries of the SADC that are converging to the CMA countries which can together form part of an expanded CMA. Hence, the analysis will shed light on the prospect for a SADC-wide monetary union. If the banking markets in most countries are already converging to the CMA countries, the prospect of monetary union is high, otherwise much work will still needs to be done. The estimation is carried out for each of the three interest rates but we retain only the significant PCs for analysis. Countries with high factor loading and with the same sign in the same PC are regarded as moving together and are thus grouped together. Following an iterative approach suggested by Jolliffe (2002), we subject such a group to further analysis. If the countries in the group are truly integrated, a further PCA should produce only one significant PC. If not, the process continues until we obtain a single dominant PC that explains most of the variation in the interest rates. The results are reported in Table 4. For each of our three interest rates, we report the eigenvalues and cumulative $\mathrm{R}^{2}$ for the full sample and the groups. Likewise, the factor loading of the first PC for the full sample and the groups are

reported. For groups where a factor loading is reported without eigenvalue and cumulative $\mathrm{R}^{2}$, such a factor loading is obtained from the other significant PCs of the full sample.

The results for the central bank rates of 12 countries are shown in Panel A of Table 4. The eigenvalues reveal that there are three significant PCs explaining about $86 \%$ of the 
variation in central bank rates, while the first PC (PC\#1) alone explains 61\%. Looking at the coefficients (factor loadings) of PC\#1 in the full sample, one can easily identify seven countries with high coefficients and equal signs. These seven countries constitute Group 1. Further estimation produces only one significant PC, which explains $79 \%$ of the variations in the central bank rates. Though Zambia appears to have a high coefficient with the same sign as the other seven countries in PC\#1, its coefficient also dominates the third PC (PC\#3) of the full sample. In addition, when Zambia's central bank rate is included in Group 1, two significant PCs are found. Hence, Zambia is excluded from Group 1. Though the factor loading for Botswana in PC\#1 moves in the same direction as those of the countries in group 1, its coefficient dominates the second PC. When adding Botswana's central bank rate to Group 1, two significant PCs are found. Hence, Botswana is removed from Group 1. In the subsequent PCA, though factor loadings for Botswana are highest, the factor loadings for Madagascar and Tanzania are equally high, but both move in the opposite direction compared to Botswana. The three countries' central bank rates are estimated, but the results produced two significant PCs, with one dominated by Botswana and the other by Madagascar and Tanzania. Hence, it is apparent that Botswana is not in same group as the other two countries. Estimation for the two countries results in one significant PC explaining $80 \%$ of the variations in rates. An outlier is Zimbabwe whose factor loading dominates the fourth PC of the full sample. Overall with regards to the central bank rates, we can identify five groups of countries that tend to move in different directions. The dominant group, Group 1 comprises the CMA countries plus Angola, Malawi and Seychelles. Group 2 comprises Madagascar and Tanzania, while the other three countries, Botswana, Zambia and Zimbabwe, are largely independent.

[Insert Table 4 about here] 
In the case of deposit rates reported in Panel $\mathrm{B}$, the results show that there are four significant PCs for the 14 countries in the sample. Following the same procedure as for central bank rates, we identify five groups of countries moving in different directions. In Group 1 there are seven countries, including the SACU members plus Seychelles and Zambia. Angola seems to move closely with Group 1 as it does not have a high factor loading outside the first PC. However, when Angola is included in Group 1, two significant PCs are found. Group 2 comprises Madagascar and Tanzania with the first PC explaining 75\% of the variations in their deposit rates. Both countries also dominate the second PC of the full sample. Group 3 includes Malawi and Mozambique. The factor loadings for Mauritius and Zimbabwe dominate the fourth PC of the full sample and they appear to move together. However, the results of their combined group indicate almost two significant PCs with the second PC explaining $41 \%$ of the variations in their deposit rates. A plot of their interest rate series also does not show any meaningful co-movement. As noted by Jolliffe (2002), it is unwise to group such countries together and we therefore treat Malawi and Mozambique as independent.

The results of the PCA for lending rates for 14 countries are reported in Panel C. The PCA detects three significant PCs accounting for $85 \%$ of the variations in the lending rates. An analysis of the first PCs shows that eight of the countries significantly move together. Further PCA can detect only one significant PC explaining about $84 \%$ of the variations in lending rates. It is also noteworthy that the eigenvalue of the second PC of the eight countries is very low (0.57). A look at the factor loading of the first PC of these countries reveals that they move together in a very uniform manner. These countries, which are the CMA members and Angola, Malawi, Seychelles and Zambia, represent Group 1. Four countries, Botswana, Madagascar, Mozambique and Tanzania, dominate the second PC of the full sample as shown by their factor loadings, but tend to move in pairs in the opposite direction. An analysis of four of them together results in two significant factors. Hence, they are analysed in pairs as suggested by the signs of their factor loadings. The PCA of the first pair of countries, Botswana and 
Mozambique, detects one significant PC that explains 85\% of the variations in their lending rates. These countries therefore represent Group 2. Group 3 comprises the second pair of countries, Madagascar and Tanzania. As the results show, the only significant PC accounts for 85\% of variations in their lending rates. Mauritius appears to dominate the third PC of the full sample and is thus independent. For Zimbabwe, the highest factor loading appears in the first PC but it moves in an opposite direction. Zimbabwe is thus also independent.

A summary of the convergence group analyses is presented in Table 5. It is evident from the results that some countries are showing signs of convergence with the CMA countries towards the end of our sample period. Prominent among them is Seychelles which converges in all three markets. Angola, Malawi and Zambia show convergence in two out of the three markets. In Botswana, convergence only occurs in the retail deposit market. A number of countries such as Madagascar, Mozambique and Tanzania seem to be moving together in pairs, but not with the CMA countries. Lastly, a few countries behave independently of the others. Notable among them are Zimbabwe and Mauritius. The central bank rates in Botswana and Zambia also behave in that fashion.

\section{[Insert Table 5 about here]}

\subsection{Driving forces of integration: Evidence from pass-through analysis}

An efficient and homogeneous PT from central bank rates onto retail banking rates can create the equivalent of an integrated banking market under the condition of a monetary integration that has already tied together policy-determined interest rates. Monetary integration is, however, neither sufficient nor necessary for banking market integration. It is not sufficient if the PT remains heterogeneous across countries because of special national characteristics of the banking systems and a lack of cross-border arbitrage. On the other hand, monetary integration may not be necessary for banking market integration as it has become clear from the 
previous PCA for the convergence club during 2000-2005. In some cases, banking markets in fact lead rather than lag integration. In these cases an indirect PT from foreign central bank rates to national retail rates may exist via the foreign retail rates. Next to the national PT we therefore also investigate the PT from South African central bank rates to national retail rates.

We conduct rolling PT analysis for all SADC countries from 1990 to 2005 using the same five-year windows as for the PCA. All national multipliers are given in Table A-2 in Appendix A. Figure 4 summarizes our findings for the national PT by showing the average long-run multipliers and their variation in SADC and the sub-regions. Our results show that the PT onto deposit rates is generally been less than perfect with the long-run multiplier dropping substantially below the full PT value of 1 to a level below 0.6. Remarkably, this is not a single phenomenon in some groups but happens in all subgroups of the SADC countries. Consequently, the coefficient of variation remains rather stable over the investigated period, suggesting that the PT-heterogeneity within both SADC and the subgroups does not change much over time. The only exemptions are the OTHER countries. Here we find episodes of increased heterogeneity in the mid-1990s and early 2000s, suggesting a sensitivity of the PT to changes in the macroeconomic and regulatory environment. With respect to the short-run multiplier or the "speed" of the PT (not shown in Figure 4) it is interesting to note that these long-run values of the average multipliers are almost reached after three months. However, the 3-months multipliers show a slightly higher variability across countries. The reduction in the $\mathrm{PT}$, in an advanced economy regularly interpreted as a signal of market imperfections, is in the SADC context predominantly linked to financial deregulation with respect to interest rates. In fact, by European standards a long-term PT of 0.6 for deposit rates is quite high. But it should also be noted that high inflation (at least above a certain threshold) typically leads to a high PT. With respect to loan rates we also find (partly for similar reasons) a reduction in the long-run average PT below the full PT-benchmark of 1 . Here, however, we clearly document a strong difference between CMA and SACU on the one hand, and the OTHER countries on the other 
hand. The former groups exhibit a still strong PT with South Africa often having a full PT, while in the latter group the PT is rather limited with an average long-run multiplier of close to 0.4 which may indicate serious banking market imperfections. Consequently, CMA and SACU have a much more homogenous PT than the others, although the heterogeneity increased somewhat during the later periods across all SADC countries.

[Insert Figure 4 about here]

In order to test for PT-differences between CMA, SACU and OTHER we adopt a simple dummy regression approach. We regress the multipliers of different time horizons on a CMA and a Botswana dummy and use additional time dummies for the rolling time periods (with the rolling period 2001-2005 as benchmark). These regressions are reported in Table 6 and reveal that the lending rate PT is significantly faster (between 0.24 and 0.35 points) and more complete (plus 0.24) in the CMA countries than in the OTHER countries. In Botswana the PT is only faster (up to 0.22 points) but not more complete. Moreover, the impression of a more efficient PT in the second half of the 1990s that can be interfered from Figure 4 is confirmed by the regression. With respect to deposit rates the PT in the CMA is speedier but not more complete. No significant effects are found for Botswana - at least at a $5 \%$ confidence level or higher.

[Insert Table 6 about here]

To highlight the drivers of banking market integration we concentrate in the following on the PT of those countries identified by the PCA in Table 5 as Group 1. As expected, all four CMA members belong to this convergence group and exhibit not only a fast but also (almost) perfect PT in lending rates. This PT is furthermore heavily influenced by South African central 
bank rates. We therefore conduct an additional PT analysis with respect to South Africa rates. The detailed results for Group 1 countries are reported in Table A-4 in Appendix A and longrun multipliers are shown in Figure 5. The long run PT in the most recent period of investigation in Namibia is 0.8 for the national policy rate and 0.72 for the South African policy rate. In Swaziland, the values are 0.93 and 0.86, respectively, while lending rates in Lesotho are directly and exclusively responding to South African rates, possibly a consequence of the financial distress in this country described earlier. With respect to deposit rates the situation is similar. The highest long-term PT is found in South Africa (0.86). In the other CMA countries the PT from national rates is only marginally faster than the PT from South African policy rates (Namibia: 0.43 versus 0.38 , Swaziland 0.74 versus 0.74 , and Lesotho is again mainly responding to the South African rate with a PT coefficient of 0.24). In sum, it appears that banking market integration is following monetary integration. Thus, South African monetary policy plays a dominant role in banking market behavior. ${ }^{11}$ The PT evidence is therefore fully in line with the PC evidence that groups all CMA countries into one convergence group for all three interest rates. The PT also finds more evidence for integration in lending rather than deposit markets, particularly for Namibia. This finding is again in line with the PC results which found least convergence in deposits.

At the other extreme we have Botswana and Zambia who also belong to Group 1 but appear to be driven by banking market integration only. This process can be completely mediated through banking market arbitrage and is as such been detect by the PCA. Potentially, capital account convertibility in both countries drives these developments. Given the fast and (almost) complete PT in South Africa, the national banking rates should then also exhibit a statistical relationship with South African policy rates. In fact, Zambia’s deposit and lending

\footnotetext{
${ }^{11}$ These findings for CMA countries are also robust when allowing for asymmetric and threshold adjustment of retail rates as reported by Sander and Kleimeier (2006).
} 
rates are increasingly related to South African central bank rates as is Botswana's deposit rate, though at a very low level.

In the remaining countries of the convergence Group 1 we find both monetary and (some) banking market integration. The Seychelles are the only non-CMA member of the Group 1 convergence club with respect to all three interest rates. As such, both an efficient national PT as well as a link to South African rates may be important. In fact, the Seychelles' lending rate is responding to the national central bank rate, as can be seen from a (small) longrun multiplier of 0.54 in the most recent rolling period, but is also co-integrated with the South African policy rate with a most recent long-run multiplier of 0.19 . The long-run responses of the deposit rate to national and South African policy rates are in a similar range with multiplier values of 0.57 and 0.19 , respectively. Angola and Malawi are in Group 1 with respect to central bank rate and lending rates. In fact, we find their lending rates to be co-integrated with the South African policy rate while deposit rates are not consistently co-integrated over time. The (too) high multipliers are however difficult to interpret and may reflect the fact that national policy rates that still largely diverge from the South African ones.

\section{[Insert Figure 5 about here]}

In sum, banking market integration can be both a genuine process and/or driven by monetary integration. However, especially banking-market driven integration needs capital account convertibility. This is not needed with monetary integration in conjunction with an efficient and homogenous PT. Moreover, the latter approach has shown its ability to improve the efficiency of national banking markets in terms of a faster and more complete PT. From a policy perspective, however, a strong competition policy is needed whenever cross-border banking is limited, be it for regulatory or economic reasons. 


\subsection{Integration and monetary policy efficiency: policy implication for an extension of CMA}

The PCA allows us to identify a convergence club within SADC. A first group within this convergence club consists of the CMA countries. They exhibit monetary integration and banking integration in loan and deposit markets. Clearly, here the monetary policy rates are dominated by South Africa. Moreover, as the PT is quite efficient in these countries, banking rates are also integrated. Consequently, the lending and deposit rates respond in a very similar way to changes in both, the domestic and the South African central bank rate. For example, the long run multiplier for loans in Namibia with respect to the domestic policy rate is 0.80 and with respect to the South African rate it is 0.72 . The corresponding responses in Swaziland are 0.93 and 0.86, respectively. The respective long-run multipliers for deposits in Namibia are 0.43 and 0.38 , and 0.74 with respect to both policy rates in Swaziland.

A second group comprises non CMA-countries with monetary and banking integration in loan and deposit markets. Again the banking market integration appears to be mediated through the South African monetary policy rate as we find the Seychelles' deposit and lending rates to be cointegrated with the South African monetary policy rate. However, the long-run PT from South Africa is here only 0.20 for both rates. Also Angola’s and Malawi’s lending rates are cointegrated with the South African Policy rate but the long run multipliers are implausibly high.

Finally there are non-CMA countries with only banking market integration and no monetary integration, such as Botswana where we find for the deposit rate and - very recently - for the lending rate some evidence for cointegration with the South African central bank rate, but only at a very low level. In a similar vein, Zambian deposit and lending rates are related to the South African central bank rate, but also at a very low level and the evidence for cointegration is weak.

In sum, we find the CMA countries ready for further integration when judged from the point of view of financial integration only. Additionally, the Seychelles show a good potential 
for joining CMA and their further deepening towards a monetary union. In fact, the Seychelles have a high financial development with respect to deposit and low interest rate spreads. They do, however, still have an underdeveloped credit market both in absolute terms and in relative terms in relation to CMA. Zambia and Botswana are also potential candidates for a CMA expansion $^{12}$ as there is already some integration evidence for banking markets. These two countries orient themselves to some extent at South African policy rates, but low levels of financial development and efficiency require particular attention of financial development and competition policies. This applies even more to Angola and Malawi who both show some signs of integration combined with even worse financial development and financial efficiency indicators. For the remaining countries, financial integration indicators do not yet point to a readiness for CMA membership.

\section{$\underline{\text { 5. Conclusions }}$}

Monetary and financial integration is high on the agenda of SADC's policy makers and has received increasing attention in the academic literature. We investigate the state, development and drivers of banking market integration in SADC member countries by employing interest rate data. Banking market integration can be a market-determined process and/or driven by monetary integration. We find evidence for increasing monetary integration and banking integration in loan and deposit markets. Integration is, however, not developing uniformly but concentrated in only a few countries. In particular, being a member of a currency area like CMA is found not only to promote banking market integration but also banking market efficiency in term of a faster and more complete PT. But this banking market integration is clearly driven by monetary integration and as such dominated be the largest CMA economy, South Africa. Nevertheless, some other countries have recently been able to join to this convergence club. Some of them are increasingly integrating themselves with these

\footnotetext{
${ }^{12}$ Buigut and Valev (2006) also favor these two countries.
} 
countries in terms of both, money and banking markets, while other countries are integrating only with respect to their banking markets. We therefore conclude that a selective and cautionary expansion of CMA is possible. Seychelles, Zambia and Botswana are potential first candidates. Such an expansion, however, requires not only more policy coordination and nominal convergence but financial market imperfections need also to be addressed. When putting monetary and financial integration high on SADCs policy agenda, policy makers will thus also have to turn financial development policies into a central part of their integration vision. 


\section{$\underline{\text { References }}$}

Adam, K., T. Jappelli, A. Menichini, M. Padula, M. Pagano, 2002. Analyse, compare, and apply alternative indicators and monitoring methodologies to measure the evolution of capital market integration in the European Union. European Commission, DirectorateGeneral for Internal Affairs, February, http://www.europa.eu.int/comm/internal_market len/update/economicreform\#docs.

Aziakpono, M., 2006a. Financial integration amongst the SACU countries: Evidence from interest rate pass-through analysis. Studies in Economics and Econometrics 30(2), 1-23.

Aziakpono, M., 2006b. Financial and monetary autonomy and interdependence between South Africa and the other SACU countries. Paper presented at the 2007 ASSA Annual Meeting in Chicago, USA.

Aziakpono, M., 2004. Financial intermediation and economic growth in a highly dependent African economy: Evidence from Lesotho. Final research report on the $17^{\text {th }}$ OSSREA Social Science Research Grant Competition.

Baele, L., A. Ferrando, P. Hördahl, E. Krylova, C. Monnet, 2004. Measuring financial integration in the euro area. Oxford Review of Economic Policy 20, 509-530.

Banerjee, A., R.L. Lumsdaine, J.H. Stock, 1992. Recursive and sequential test of the unit-root and trend-break hypotheses: Theory and international evidence. Journal of Business \& Economic Statistics 10, 271-287.

Becker, B., S.G. Hall, 2007. A new look at economic convergence in Europe: A common factor approach. Loughborough University, Department of Economics, Discussion Paper Series 2007_09, http://ideas.repec.org/p/lbo/lbowps/2007 09.html.

Buigut, S.K., N.T. Valev, 2006. Eastern and Southern Africa monetary integration: A structural vector autoregression analysis. Review of Development Economics 10(4), 586-603. 
Cooper, R.N., 1971. Towards an international capital market? In Kindleberger, P.C., and Schonfield, A (Eds), North American and Western European Economics Policies. New York: St. Martin’s Press, 192-208.

Engle, R.F., C.W.J. Granger, 1987. Co-integration and error correction: Representation estimation and testing. Econometrica 55, 251-276.

Fernandez-Izquierdo, A., J.A. Lafuente, 2004. International transmission of stock exchange volatility: empirical evidence from the Asian crisis. Global Finance Journal 15, 125-137.

Figueira, C., J.G. Nellis, D. Parker, 2005. Testing for international financial markets integration. The Cranfield School of Management Working Paper Series, SWP 2/05, https://dspace.lib.cranfield.ac.uk/bitstream/1826/852/2/SWP0205.pdf

Gilmore, C.G., B.M. Lucey, G.M. McManus, 2007. The dynamics of Central European equity market comovements. Forthcoming in Quarterly Review of Economics and Finance.

Jenkins, C., L. Thomas, 1998. Is Southern Africa ready for Regional Monetary Integration in Petersson L.(ed.) Post-Apartheid Southern Africa: Economic Challenges and Policies for the future, London: Routledge. Chapter 8, pp.145-170.

Jolliffe, I.T., 2002. Principal Component Analysis. New York: Springer. Second Edition.

Kabundi, A., E. Loots, 2007. Co-movement between South Africa and SADC countries: Is trade a predominant factor? Forthcoming in Economic Modelling.

Kaiser, H.F., 1960. The application of electronic computers to factor analysis. Educational and Psychological Measurement 20, 141-151.

Kleimeier, S., H. Sander, 2007. Integrating Europe’s retail banking markets: Where do we stand? CEPS Research Report on Finance and Banking, Centre for European Policy Studies, Brussels, http://shop.ceps.eu/BookDetail.php?item id=1548. 
Kleimeier, S., H. Sander, 2000. Regionalisation versus globalisation in European financial market integration: Evidence from co-integration analysis. Journal of Banking and Finance 24, 1005-1043.

Kremers, J.J.M., N.R. Ericsson, J.J. Dolado, 1992. The power of cointegration tests, Oxford Bulletin of Economics and Statistics 54(3), 325-348.

Logue, D.E., M.A. Salant, R.J. Sweeney, 1976. International integration of financial markets: survey, synthesis and results. In Stern, C.H. et al., (Eds) Eurocurrencies and the International Monetary System. Washington DC: American Enterprise for Public Policy Research, 91-138.

McCarthy, C.L., 2002. Macroeconomic convergence in SADC - a policy perspective for the central banks of the integration arrangement. Study report submitted to the Committee of Central Bank Governors in SADC, http://www.sarpn.org.za/documents/d0000559 /P512_Macro-economic_SADC.pdf.

Meric, I., M. Ratner, G. Meric, 2007. Co-movements of sector index returns in the world's major stock markets in bull and bear markets: Portfolio diversification implications. Forthcoming in International Review of Financial Analysis.

Nellis, J.G., 1982. A principal components analysis of international financial integration under fixed and floating exchange rate regimes. Applied Economics, 14, 339-354

Nielsen, H., E. Uanguta, S. Ikhide, 2005. Financial integration in the common monetary area. South African Journal of Economics 73(4), 710-721.

Perignon, C., D.R. Smith, C. Villa, 2007. Why common factors in international bond returns are not so common. Journal of International Money and Finance 26, 284-304.

Rose, A.K., 2001. Currency unions and trade: The effect is large, Economic Policy 33, October, 449-461.

Rossouw, J., 2006. An analysis of macro-economic convergence in SADC. South African Journal of Economics 74(3), 382-390. 
Sander, H., S. Kleimeier, 2004. Convergence in Eurozone retail banking? What interest rate pass-through tells us about monetary policy transmission, competition and integration. Journal of International Money and Finance 23, 461-492.

Sander, H., S. Kleimeier, 2006. Interest rate pass-through in the Common Monetary Area of the SACU countries. South African Journal of Economics 74(2), 215-229.

Siliverstovs, B., G. L’Hegaret, A. Neumann, C. von Hirschhausen, 2005. International market integration for natural gas? A cointegration analysis of prices in Europe, North America and Japan. Energy Economics 27, 603-615.

\section{$\underline{\text { Appendix A }}$}

[Insert Tables A-1 and A-4 here]

\section{Appendix B}

[Insert Tables B-1 and B-8 here] 


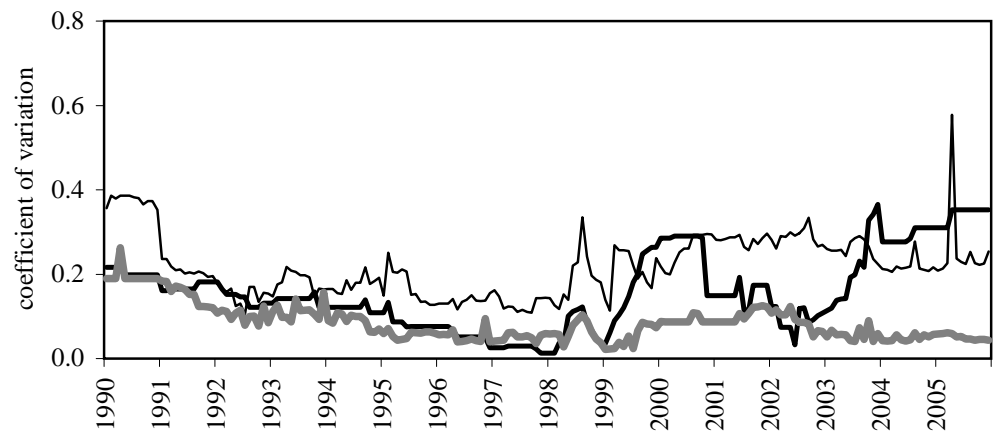

Panel B: SACU

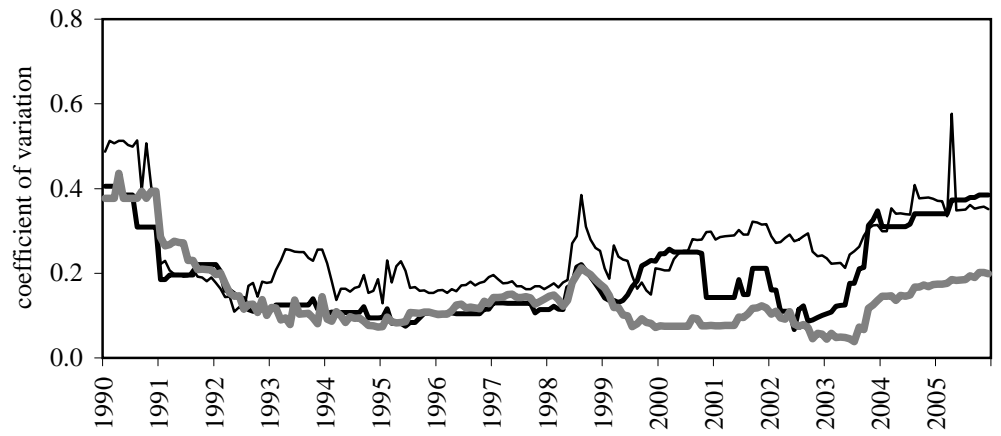

Panel C: OTHER

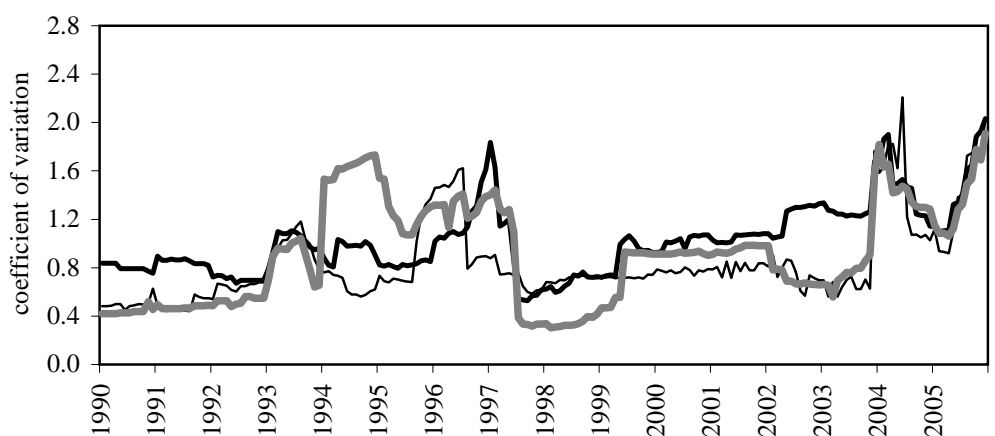

Panel D: SADC

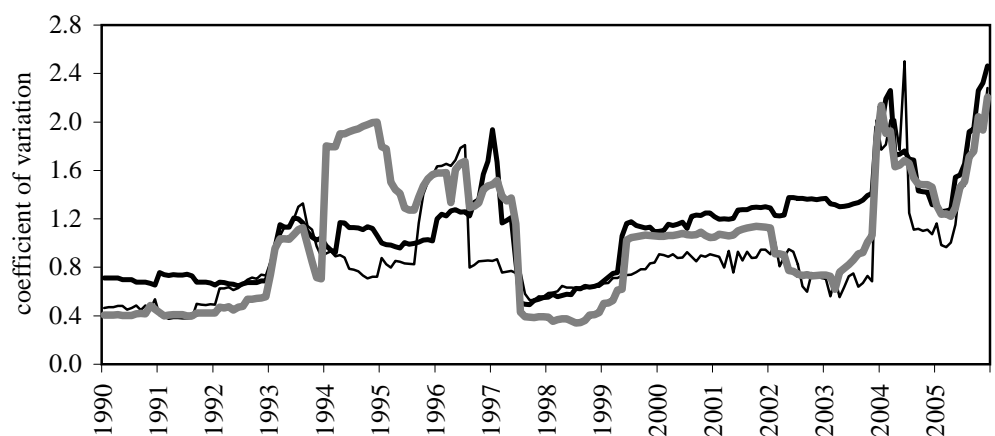

—bank rate - $\quad$ deposit rate lending rate

Note: The regional groups comprise the following countries: CMA = Lesotho, Namibia, South Africa, Swaziland; SACU = all CMA countries and Botswana; OTHER = Angola, DR Congo, Madagaska, Malawi, Mauritius, Mozambique, Seychelles, Tanzania, Zambia, Zimbabwe; SADC = all OTHER and SACU countries. The coefficient of variation is defined as the standard deviation divided by the average of all interest rates in the region. 

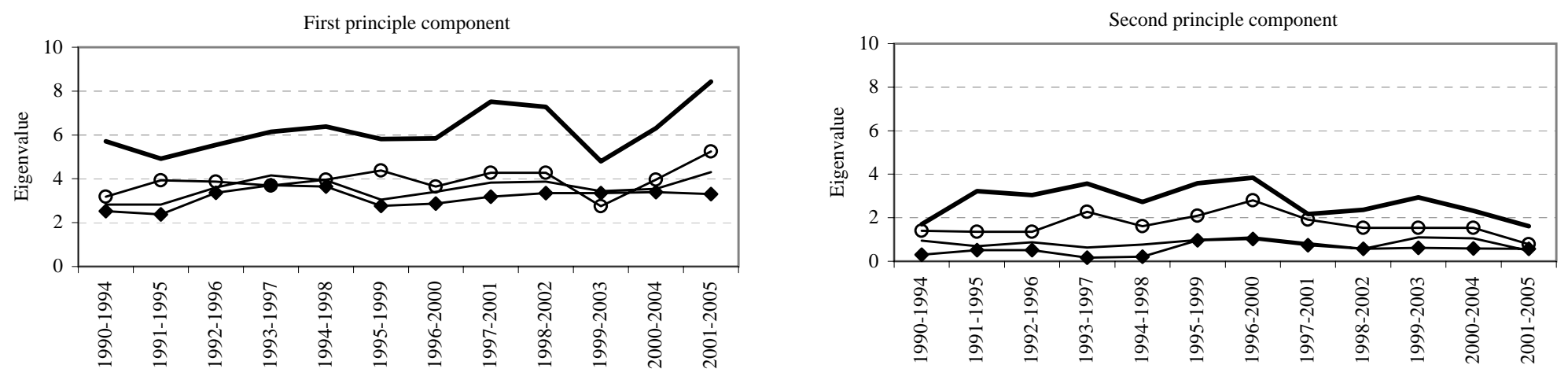

Panel B: Deposit rates
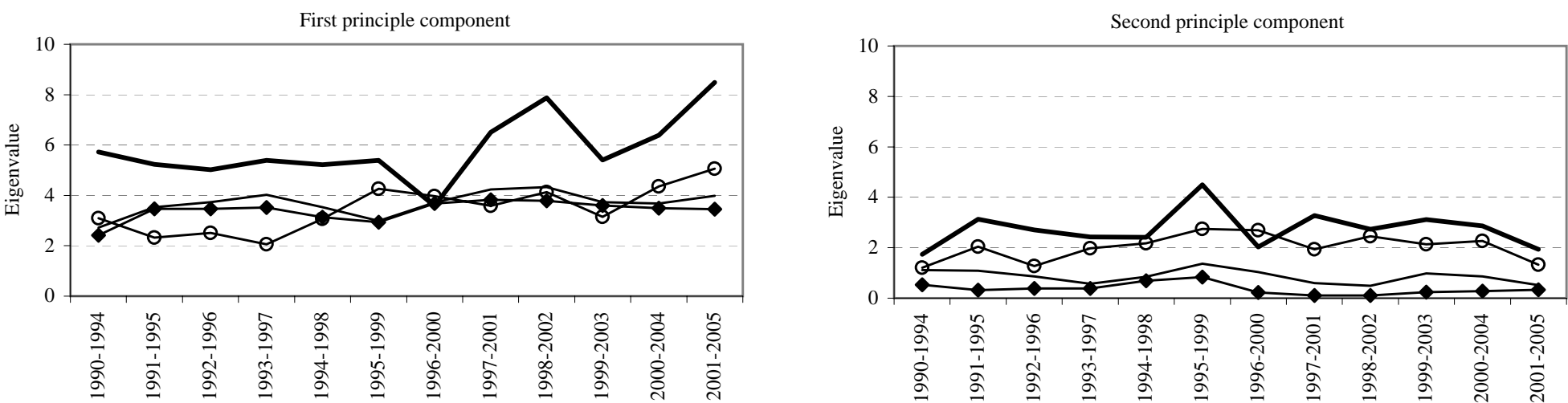

Panel C: Lending rates
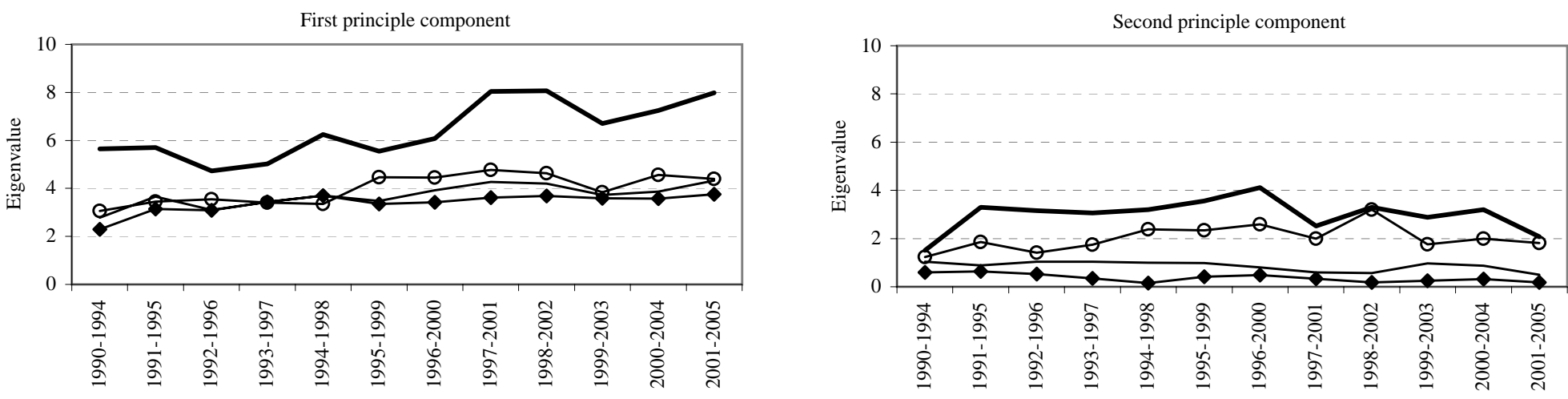

$\multimap \mathrm{CMA} \longrightarrow \mathrm{SACU} \multimap$ OTHERS $\longrightarrow$ SADC 
Figure 3: First factor loading as indicator of financial integration
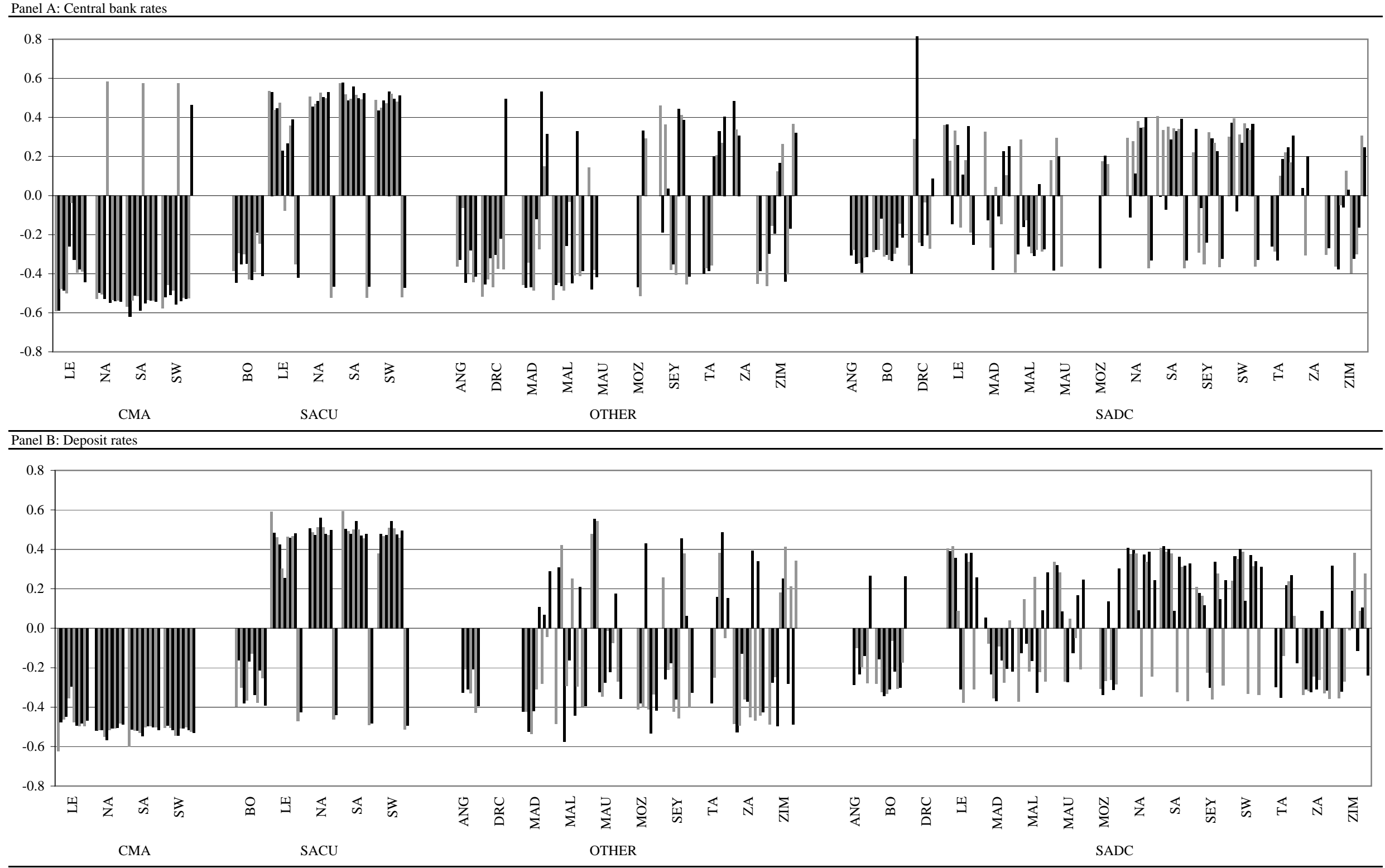
Figure 3: First factor loading as indicator of financial integration

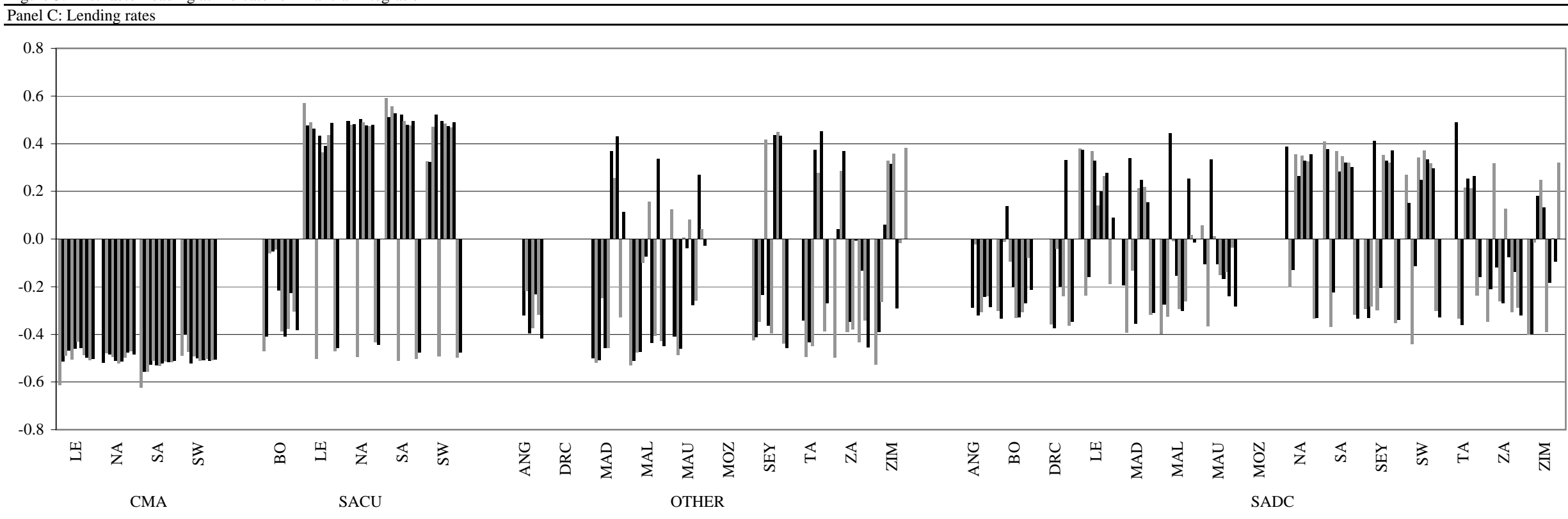

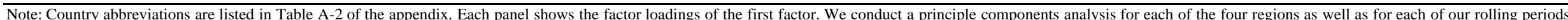

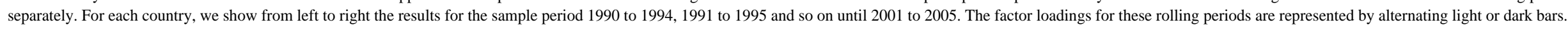



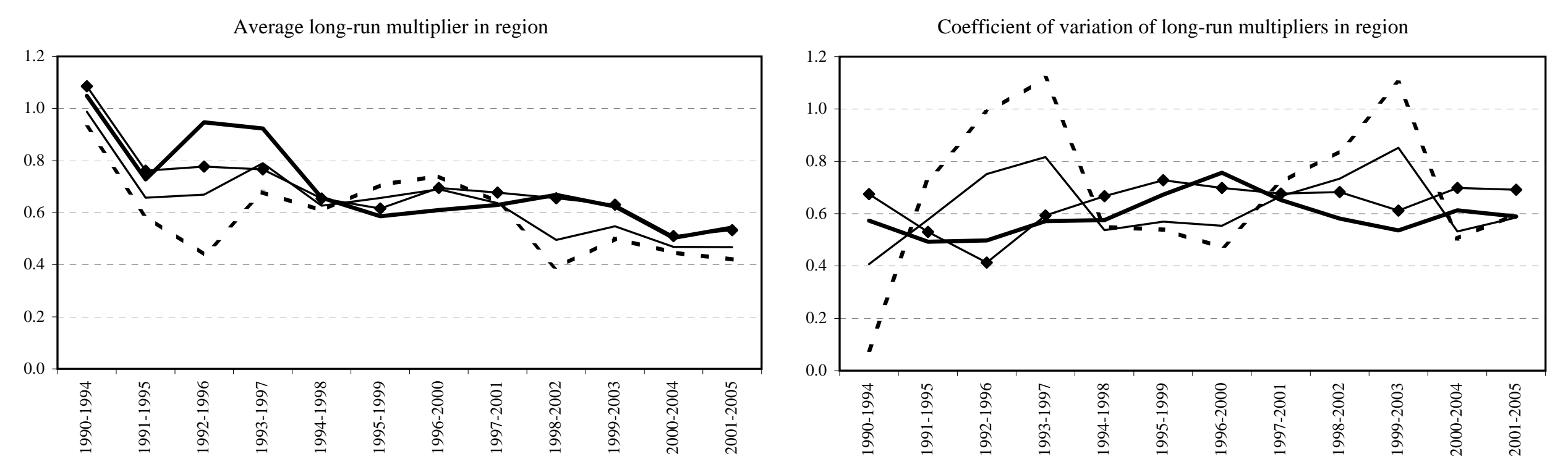

Panel B: Lending rates

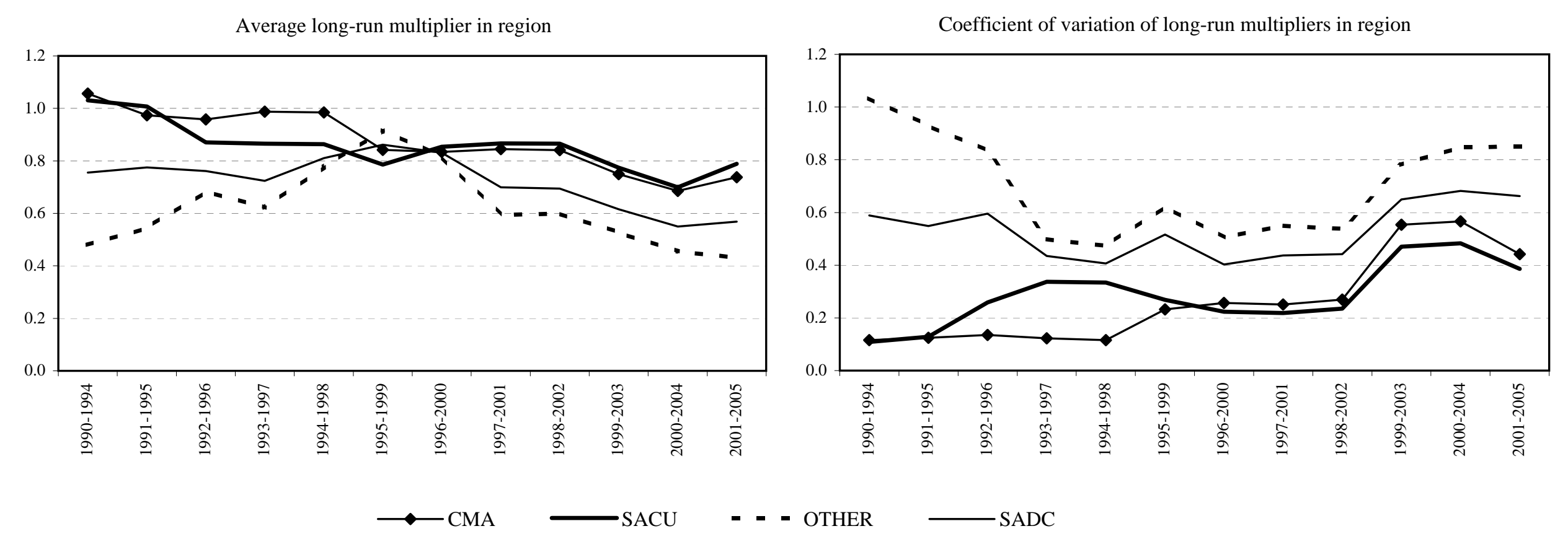

Note: For the country-composition of the regions, see notes to Figure 1. 
Long-run multiplier

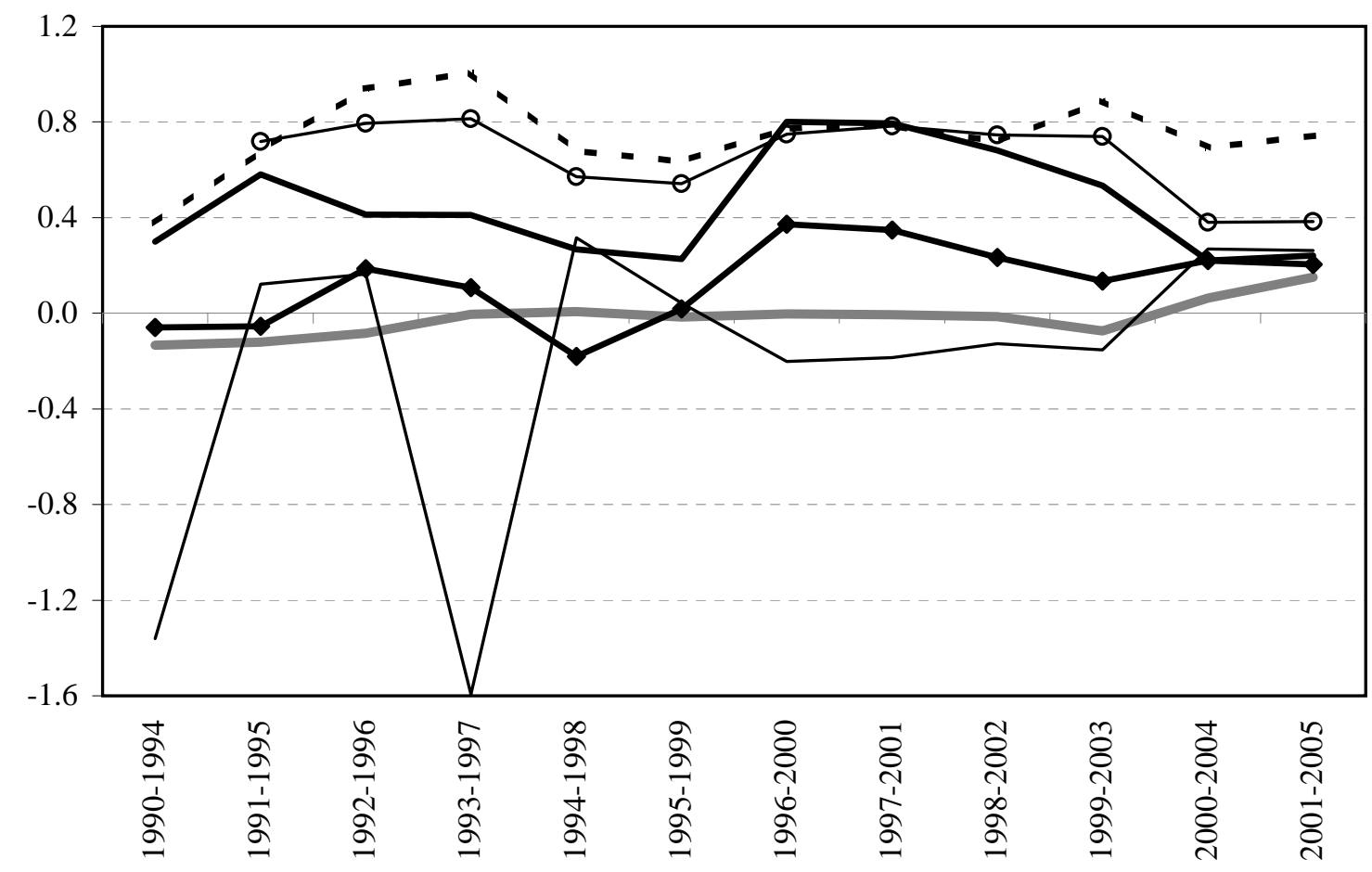

Panel B: Lending rates

Long-run multiplier

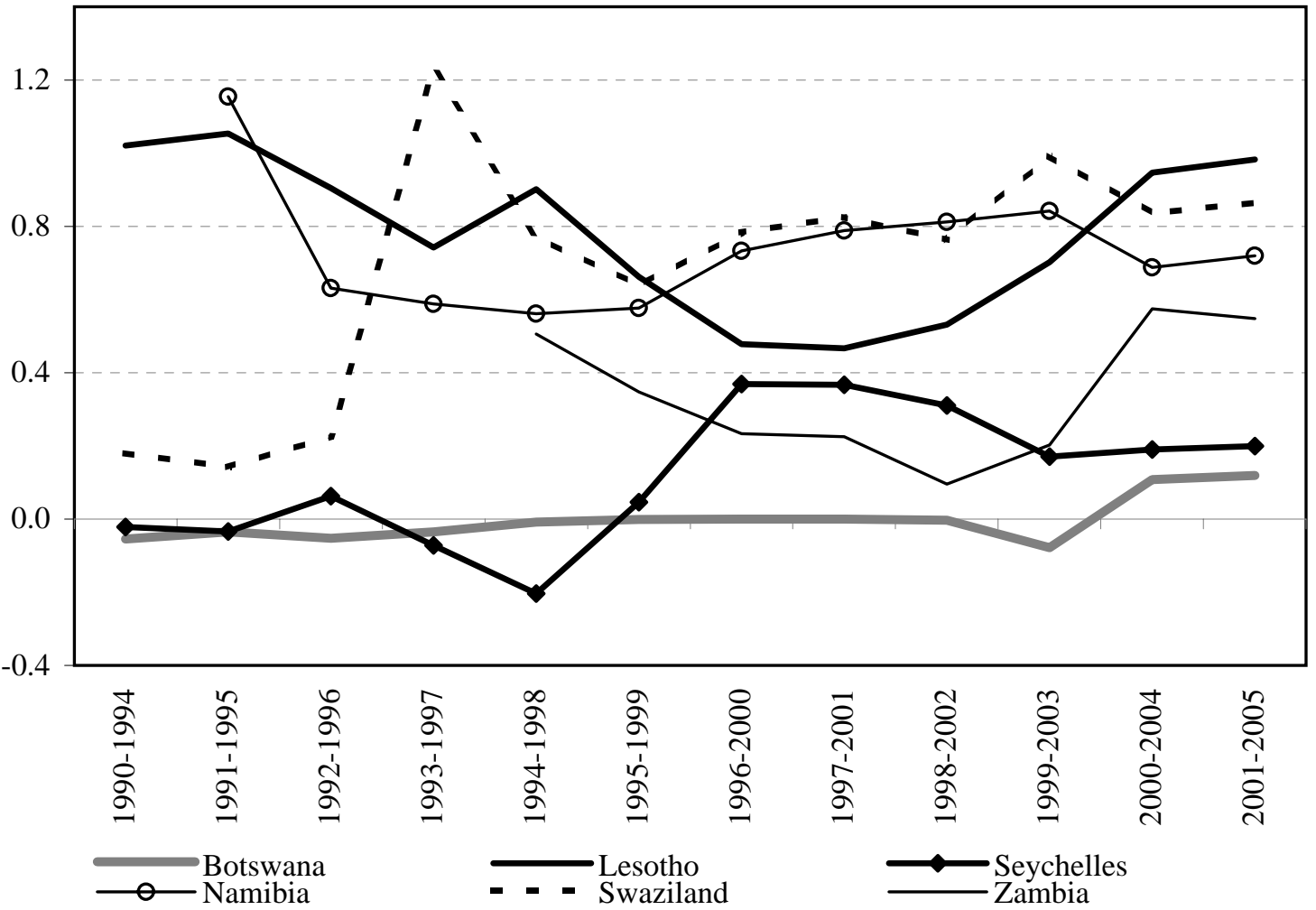

Note: For the country-composition of the regions, see notes to Figure 1. 
Table 1: Economic conditions and financial development

\begin{tabular}{|c|c|c|c|c|c|c|c|c|c|c|c|c|c|c|c|c|c|c|}
\hline & Lesotho & Namibia & $\begin{array}{r}\text { South } \\
\text { Africa } \\
\end{array}$ & $\begin{array}{r}\text { Swazi- } \\
\text { land } \\
\end{array}$ & CMU & Botswana & SACU & Angola & $\begin{array}{l}\text { Mada- } \\
\text { gascar }\end{array}$ & Malawi & Mauritius & $\begin{array}{r}\text { Mozam- } \\
\text { bique }\end{array}$ & $\begin{array}{r}\text { Sey- } \\
\text { chelles }\end{array}$ & Tanzania & Zambia & $\begin{array}{c}\text { Zim- } \\
\text { babwe }\end{array}$ & OTHERS & SADC \\
\hline \multicolumn{19}{|c|}{ Panel A: Average annual real GDP growth } \\
\hline 1990 to 1994 & $4.4 \%$ & $4.4 \%$ & $0.2 \%$ & $3.9 \%$ & $0.4 \%$ & $4.4 \%$ & $0.6 \%$ & $-5.9 \%$ & $0.0 \%$ & $1.3 \%$ & $5.3 \%$ & $4.4 \%$ & $4.5 \%$ & $2.5 \%$ & $-0.8 \%$ & $2.8 \%$ & $1.6 \%$ & $0.9 \%$ \\
\hline 1995 to 1999 & $3.6 \%$ & $3.6 \%$ & $2.6 \%$ & $3.7 \%$ & $2.6 \%$ & $8.0 \%$ & $2.8 \%$ & $7.9 \%$ & $3.2 \%$ & $6.9 \%$ & $4.9 \%$ & & $3.4 \%$ & $3.8 \%$ & $1.5 \%$ & $2.5 \%$ & $3.7 \%$ & $3.1 \%$ \\
\hline 2000 to 2005 & $2.6 \%$ & $4.6 \%$ & $3.9 \%$ & $1.9 \%$ & $3.9 \%$ & $5.3 \%$ & $3.9 \%$ & $9.3 \%$ & $2.9 \%$ & $2.6 \%$ & $4.2 \%$ & & $1.2 \%$ & $6.5 \%$ & $4.6 \%$ & $-6.1 \%$ & $1.9 \%$ & $3.4 \%$ \\
\hline \multicolumn{19}{|c|}{ Panel B: Average annual inflation rate } \\
\hline 1990 to 1994 & $13.6 \%$ & $12.2 \%$ & $12.4 \%$ & $11.1 \%$ & $12.4 \%$ & $12.8 \%$ & $12.4 \%$ & $677.7 \%$ & $16.8 \%$ & $21.1 \%$ & $8.6 \%$ & $46.2 \%$ & $2.5 \%$ & $29.0 \%$ & $121.7 \%$ & $26.5 \%$ & $115.0 \%$ & $42.1 \%$ \\
\hline 1995 to 1999 & $8.1 \%$ & $8.3 \%$ & $7.3 \%$ & $8.0 \%$ & $7.4 \%$ & $8.7 \%$ & $7.4 \%$ & $1478.3 \%$ & $17.9 \%$ & $40.9 \%$ & $6.6 \%$ & $22.9 \%$ & $1.6 \%$ & $17.2 \%$ & $30.7 \%$ & $30.6 \%$ & $210.1 \%$ & $66.1 \%$ \\
\hline 2000 to 2005 & $7.6 \%$ & $7.2 \%$ & $5.1 \%$ & $7.6 \%$ & $5.3 \%$ & $8.0 \%$ & $5.4 \%$ & $126.1 \%$ & $11.0 \%$ & $17.2 \%$ & $4.9 \%$ & $12.0 \%$ & $3.4 \%$ & $4.0 \%$ & $21.2 \%$ & $90.9 \%$ & $45.2 \%$ & $16.9 \%$ \\
\hline \multicolumn{19}{|c|}{ Panel C: Average annual spread between lending and deposit rate } \\
\hline 1990 to 1994 & $7.1 \%$ & $8.9 \%$ & $3.9 \%$ & $6.0 \%$ & $4.1 \%$ & $1.7 \%$ & $4.0 \%$ & & $5.8 \%$ & $7.1 \%$ & $6.8 \%$ & & $6.3 \%$ & $14.5 \%$ & $16.3 \%$ & $2.1 \%$ & $5.7 \%$ & $4.5 \%$ \\
\hline 1995 to 1999 & $6.8 \%$ & $7.5 \%$ & $4.9 \%$ & $7.4 \%$ & $5.0 \%$ & $4.7 \%$ & $5.0 \%$ & $42.3 \%$ & $15.8 \%$ & $15.3 \%$ & $9.6 \%$ & $14.5 \%$ & $6.5 \%$ & $17.2 \%$ & $17.1 \%$ & $12.2 \%$ & $17.7 \%$ & $8.7 \%$ \\
\hline 2000 to 2005 & $10.4 \%$ & $6.2 \%$ & $4.9 \%$ & $7.0 \%$ & $5.0 \%$ & $6.0 \%$ & $5.0 \%$ & $58.6 \%$ & $11.9 \%$ & $22.1 \%$ & $12.0 \%$ & $10.4 \%$ & $6.5 \%$ & $12.4 \%$ & $19.7 \%$ & $73.7 \%$ & $34.5 \%$ & $13.6 \%$ \\
\hline \multicolumn{19}{|c|}{ Panel D: Average annual credit to GDP } \\
\hline 1990 to 1994 & $17.8 \%$ & $33.0 \%$ & $44.5 \%$ & $23.1 \%$ & $43.4 \%$ & $13.6 \%$ & $42.5 \%$ & & $16.3 \%$ & $10.9 \%$ & $38.5 \%$ & $12.5 \%$ & $7.9 \%$ & $11.6 \%$ & $6.2 \%$ & $17.4 \%$ & $14.8 \%$ & $34.5 \%$ \\
\hline 1995 to 1999 & $17.6 \%$ & $46.9 \%$ & $64.1 \%$ & $16.5 \%$ & $62.0 \%$ & $12.5 \%$ & $60.5 \%$ & $3.9 \%$ & $9.7 \%$ & $4.9 \%$ & $50.8 \%$ & $13.0 \%$ & $13.4 \%$ & $4.5 \%$ & $7.6 \%$ & $23.1 \%$ & $16.2 \%$ & $47.7 \%$ \\
\hline 2000 to 2005 & $10.2 \%$ & $47.9 \%$ & $67.3 \%$ & $14.8 \%$ & $64.9 \%$ & $18.4 \%$ & $63.5 \%$ & $4.4 \%$ & $9.0 \%$ & $5.9 \%$ & $64.1 \%$ & $13.0 \%$ & $24.0 \%$ & $7.0 \%$ & $7.0 \%$ & $20.3 \%$ & $17.5 \%$ & $50.2 \%$ \\
\hline \multicolumn{19}{|c|}{ Panel E: Average annual deposits to GDP } \\
\hline 1990 to 1994 & $33.6 \%$ & $31.1 \%$ & $47.1 \%$ & $27.8 \%$ & $46.1 \%$ & $19.4 \%$ & $45.3 \%$ & & $15.2 \%$ & $16.4 \%$ & $61.4 \%$ & $21.3 \%$ & $42.5 \%$ & $15.3 \%$ & $14.6 \%$ & $17.6 \%$ & $20.1 \%$ & $38.0 \%$ \\
\hline 1995 to 1999 & $30.7 \%$ & $42.0 \%$ & $51.0 \%$ & $23.1 \%$ & $49.8 \%$ & $23.7 \%$ & $49.0 \%$ & $16.0 \%$ & $13.9 \%$ & $12.9 \%$ & $71.2 \%$ & $18.3 \%$ & $73.3 \%$ & $14.3 \%$ & $14.9 \%$ & $21.8 \%$ & $23.7 \%$ & $41.7 \%$ \\
\hline 2000 to 2005 & $24.7 \%$ & $39.9 \%$ & $53.7 \%$ & $18.9 \%$ & $52.2 \%$ & $27.1 \%$ & $51.4 \%$ & $14.9 \%$ & $15.5 \%$ & $15.7 \%$ & $81.5 \%$ & $24.5 \%$ & $95.8 \%$ & $17.0 \%$ & $18.5 \%$ & $30.0 \%$ & $28.7 \%$ & $44.8 \%$ \\
\hline
\end{tabular}


Table 2: Number of factors that explain the joint variation of the national interest rates

\begin{tabular}{|c|c|c|c|c|}
\hline \multirow[b]{2}{*}{ Period } & \multicolumn{4}{|c|}{ Number of factors } \\
\hline & CMA & SACU & OTHER & SADC \\
\hline \multicolumn{5}{|c|}{ Panel A: Bank rates } \\
\hline 1990-1994 & 1 & 1 & 2 & $\overline{2}$ \\
\hline 1991-1995 & 1 & 1 & 2 & 3 \\
\hline 1992-1996 & 1 & 1 & 2 & 3 \\
\hline 1993-1997 & 1 & 1 & 2 & 3 \\
\hline 1994-1998 & 1 & 1 & 2 & 3 \\
\hline 1995-1999 & 1 & 1 & 2 & 3 \\
\hline 1996-2000 & 2 & 2 & 2 & 3 \\
\hline 1997-2001 & 1 & 1 & 3 & 3 \\
\hline 1998-2002 & 1 & 1 & 2 & 3 \\
\hline 1999-2003 & 1 & 2 & 3 & 3 \\
\hline 2000-2004 & 1 & 2 & 2 & 3 \\
\hline 2001-2005 & 1 & 1 & 1 & 2 \\
\hline \multicolumn{5}{|c|}{$\begin{array}{l}\text { Panel B: Deposit rates } \\
\end{array}$} \\
\hline 1990-1994 & 1 & 2 & 2 & 2 \\
\hline 1991-1995 & 1 & 2 & 2 & 3 \\
\hline 1992-1996 & 1 & 1 & 2 & 2 \\
\hline 1993-1997 & 1 & 1 & 3 & 3 \\
\hline 1994-1998 & 1 & 1 & 2 & 3 \\
\hline 1995-1999 & 1 & 2 & 2 & 3 \\
\hline 1996-2000 & 1 & 2 & 2 & 2 \\
\hline 1997-2001 & 1 & 1 & 3 & 3 \\
\hline 1998-2002 & 1 & 1 & 2 & 3 \\
\hline 1999-2003 & 1 & 1 & 3 & 4 \\
\hline 2000-2004 & 1 & 1 & 3 & 4 \\
\hline 2001-2005 & 1 & 1 & 2 & 3 \\
\hline \multicolumn{5}{|c|}{$\begin{array}{l}\text { Panel C: Lending rates } \\
\end{array}$} \\
\hline 1990-1994 & 1 & 2 & 2 & 3 \\
\hline 1991-1995 & 1 & 1 & 2 & 2 \\
\hline 1992-1996 & 1 & 2 & 2 & 3 \\
\hline 1993-1997 & 1 & 2 & 2 & 3 \\
\hline 1994-1998 & 1 & 2 & 3 & 3 \\
\hline 1995-1999 & 1 & 1 & 2 & 3 \\
\hline $1996-2000$ & 1 & 1 & 2 & 3 \\
\hline 1997-2001 & 1 & 1 & 2 & 3 \\
\hline 1998-2002 & 1 & 1 & 3 & 3 \\
\hline 1999-2003 & 1 & 1 & 3 & 4 \\
\hline 2000-2004 & 1 & 1 & 3 & 3 \\
\hline 2001-2005 & 1 & 1 & 2 & 3 \\
\hline
\end{tabular}

Note: The number of factors are determined via principal component analysis and any factor with an eigen value larger than 1 is taken into account. For the country-composition of the regions, see notes to Figure 1. 
Table 3: The explanatory power of the first principle component

\begin{tabular}{|c|c|c|c|c|}
\hline \multirow[b]{2}{*}{ Period } & \multicolumn{4}{|c|}{ Cumulative $\mathrm{R}^{2}$ of the first principle component } \\
\hline & CMA & SACU & OTHER & SADC \\
\hline \multicolumn{5}{|c|}{ Panel A: Bank rates } \\
\hline 1990-1994 & 0.841 & 0.706 & 0.637 & 0.635 \\
\hline 1991-1995 & 0.792 & 0.705 & 0.656 & 0.492 \\
\hline 1992-1996 & 0.840 & 0.721 & 0.554 & 0.462 \\
\hline 1993-1997 & 0.928 & 0.831 & 0.461 & 0.472 \\
\hline 1994-1998 & 0.912 & 0.787 & 0.565 & 0.532 \\
\hline 1995-1999 & 0.692 & 0.610 & 0.547 & 0.448 \\
\hline 1996-2000 & 0.717 & 0.680 & 0.456 & 0.450 \\
\hline 1997-2001 & 0.798 & 0.767 & 0.535 & 0.578 \\
\hline 1998-2002 & 0.838 & 0.773 & 0.534 & 0.560 \\
\hline 1999-2003 & 0.838 & 0.688 & 0.393 & 0.399 \\
\hline 2000-2004 & 0.847 & 0.710 & 0.565 & 0.526 \\
\hline 2001-2005 & 0.825 & 0.862 & 0.750 & 0.702 \\
\hline Average & 0.822 & 0.737 & 0.554 & 0.521 \\
\hline \multicolumn{5}{|c|}{ Panel B: Deposit rate } \\
\hline 1990-1994 & 0.807 & 0.680 & 0.620 & 0.635 \\
\hline 1991-1995 & 0.866 & 0.706 & 0.386 & 0.476 \\
\hline 1992-1996 & 0.866 & 0.746 & 0.418 & 0.456 \\
\hline 1993-1997 & 0.881 & 0.806 & 0.343 & 0.490 \\
\hline 1994-1998 & 0.784 & 0.706 & 0.437 & 0.435 \\
\hline 1995-1999 & 0.735 & 0.599 & 0.474 & 0.385 \\
\hline $1996-2000$ & 0.919 & 0.744 & 0.442 & 0.710 \\
\hline 1997-2001 & 0.955 & 0.847 & 0.399 & 0.465 \\
\hline 1998-2002 & 0.946 & 0.867 & 0.459 & 0.563 \\
\hline 1999-2003 & 0.901 & 0.746 & 0.350 & 0.386 \\
\hline 2000-2004 & 0.874 & 0.736 & 0.484 & 0.457 \\
\hline 2001-2005 & 0.863 & 0.798 & 0.632 & 0.606 \\
\hline Average & 0.866 & 0.748 & 0.454 & 0.505 \\
\hline \multicolumn{5}{|c|}{ Panel C: Lending rate } \\
\hline $1990-1994$ & 0.767 & 0.694 & 0.611 & 0.627 \\
\hline 1991-1995 & 0.784 & 0.732 & 0.574 & 0.518 \\
\hline 1992-1996 & 0.773 & 0.620 & 0.590 & 0.429 \\
\hline 1993-1997 & 0.859 & 0.688 & 0.487 & 0.419 \\
\hline 1994-1998 & 0.924 & 0.740 & 0.420 & 0.480 \\
\hline 1995-1999 & 0.839 & 0.695 & 0.497 & 0.397 \\
\hline 1996-2000 & 0.854 & 0.786 & 0.495 & 0.434 \\
\hline $1997-2001$ & 0.903 & 0.854 & 0.530 & 0.574 \\
\hline 1998-2002 & 0.920 & 0.841 & 0.463 & 0.538 \\
\hline 1999-2003 & 0.896 & 0.746 & 0.480 & 0.479 \\
\hline 2000-2004 & 0.894 & 0.773 & 0.506 & 0.518 \\
\hline 2001-2005 & 0.939 & 0.864 & 0.549 & 0.614 \\
\hline Average & 0.863 & 0.753 & 0.517 & 0.502 \\
\hline
\end{tabular}

Note: For the country-composition of the regions, see notes to

Figure 1. 
Table 4: Principal components analysis of convergence groups among SADC countries for 2000 to 2005

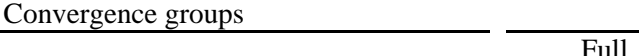

Convergence groups

Convergence groups

Full $\quad$ Convergence groups

sample Group 1 Group 2 Group 3 Group 4 Group 5 sample Group 1 Group 2 Group 3 Group 4 Group 5 Full Panel B: Deposit rate

sample Group 1 Group 2 Group 3 Group 4 Group 5 Panel A: Bank rate Panel C: Lending rate

\begin{tabular}{|c|c|c|c|c|c|c|c|c|c|c|c|c|c|c|c|c|c|c|}
\hline \multirow{2}{*}{\multicolumn{19}{|c|}{ Eigenvalue }} \\
\hline & & & & & & & & & & & & & & & & & & \\
\hline PC\#1 & 7.357 & 5.532 & 1.604 & na & na & na & 7.727 & 4.81 & 1.506 & 1.516 & na & na & 7.999 & 6.683 & 1.685 & 1.691 & na & na \\
\hline PC\#2 & 1.798 & 0.772 & 0.396 & na & na & na & 2.106 & 0.842 & 0.494 & 0.484 & na & na & 2.826 & 0.569 & 0.316 & 0.309 & na & na \\
\hline PC\#3 & 1.152 & na & na & na & na & na & 1.316 & 0.664 & na & na & na & na & 1.028 & 0.352 & & & na & na \\
\hline PC\#4 & 0.663 & na & na & na & na & na & 1.031 & na & na & na & na & na & 0.827 & & & & na & na \\
\hline \multicolumn{19}{|c|}{ Cumulative $R^{2}$} \\
\hline PC\#1 & 0.613 & 0.791 & 0.802 & na & na & na & 0.552 & 0.687 & 0.753 & 0.758 & na & na & 0.571 & 0.835 & 0.842 & 0.846 & na & na \\
\hline PC\#2 & 0.763 & 0.901 & 1.000 & na & na & na & 0.702 & 0.807 & 1 & 1 & na & na & 0.773 & 0.907 & 1.000 & 1.000 & na & na \\
\hline PC\#3 & 0.859 & na & na & na & na & na & 0.796 & 0.902 & na & na & na & na & 0.847 & 0.951 & na & na & na & na \\
\hline PC\#4 & 0.914 & na & na & na & na & na & 0.871 & na & na & na & na & na & 0.906 & na & na & na & na & na \\
\hline \multicolumn{19}{|c|}{ Factor loading of vector 1} \\
\hline Angola & -0.329 & -0.358 & & & & & -0.282 & & & & & -0.282 & -0.278 & -0.312 & & & & \\
\hline Botswana & -0.129 & & & 0.512 & & & -0.237 & -0.296 & & & & & -0.112 & & 0.707 & & & \\
\hline Lesotho & -0.205 & -0.289 & & & & & -0.263 & -0.368 & & & & & -0.349 & -0.379 & & & & \\
\hline Madagascar & 0.219 & & 0.707 & & & & 0.201 & & 0.707 & & & & -0.039 & & & 0.707 & & \\
\hline Malawi & -0.303 & -0.351 & & & & & -0.284 & & & 0.707 & & & -0.319 & -0.352 & & & & \\
\hline Mauritius & & & & & & & -0.246 & & & & -0.587 & & 0.029 & & & & 0.933 & \\
\hline Mozambique & & & & & & & -0.296 & & & 0.707 & & & -0.156 & & 0.707 & & & \\
\hline Namibia & -0.348 & -0.413 & & & & & -0.249 & -0.375 & & & & & -0.329 & -0.364 & & & & \\
\hline Seychelles & -0.351 & -0.414 & & & & & -0.341 & -0.301 & & & & & -0.328 & -0.358 & & & & \\
\hline South Africa & -0.344 & -0.401 & & & & & -0.254 & -0.444 & & & & & -0.335 & -0.371 & & & & \\
\hline Swaziland & -0.346 & -0.403 & & & & & -0.323 & -0.443 & & & & & -0.321 & -0.351 & & & & \\
\hline Tanzania & 0.223 & & 0.707 & & & & 0.066 & & 0.707 & & & & -0.203 & & & 0.707 & & \\
\hline Zambia & -0.296 & & & & 0.416 & & -0.336 & -0.401 & & & & & -0.312 & -0.338 & & & & \\
\hline Zimbabwe & 0.272 & & & & & -0.649 & 0.252 & & & & & -0.455 & 0.301 & & & & & 0.301 \\
\hline
\end{tabular}

Note: na denotes not applicable. Bank rate was not available for DR Congo, Mauritius and Mozambique. Deposit and Lending rates were not available for DR Congo. 
Table 5: Principal components analysis of convergence groups among SADC countries from 2000 to 2005

\begin{tabular}{lccc}
\hline & Group 1 & Group 2 & Group 3 \\
\hline Angola & BL & D \\
$\begin{array}{l}\text { Botswana } \\
\text { Lesotho } \\
\text { Madagascar }\end{array}$ & D & L & B \\
$\begin{array}{l}\text { Malawi } \\
\text { Mauritius }\end{array}$ & BL & BDL \\
$\begin{array}{l}\text { Mozambique } \\
\text { Namibia }\end{array}$ & BDL & DL & DL \\
$\begin{array}{l}\text { Seychelles } \\
\text { South Africa }\end{array}$ & BDL & \\
$\begin{array}{l}\text { Swaziland } \\
\text { Tanzania }\end{array}$ & BDL & \\
$\begin{array}{l}\text { Zambia } \\
\text { Zimbabwe }\end{array}$ & DL & \\
$\begin{array}{l}\text { Note: B - Bank rate, D - Deposit rate and L - Lending } \\
\text { rate; Group 2- Pair of countries move together and } \\
\text { Group 3- Each country is independent. }\end{array}$
\end{tabular}


Table 6: Region and time patterns in the national pass-through in SADC banking markets

\begin{tabular}{|c|c|c|c|c|c|c|c|c|c|c|c|c|}
\hline \multirow[b]{2}{*}{ Independent variable } & \multicolumn{6}{|c|}{ Dependent variable: Multiplier for a $+1 \%$ shock } & \multicolumn{6}{|c|}{ Dependent variable: Multiplier for a $+1 \%$ shock } \\
\hline & Impact & 1 month & 3 months & 6 months & 12 months & long-run & Impact & 1 month & 3 months & 6 months & 12 months & long-run \\
\hline & \multicolumn{6}{|c|}{ Panel A: Loan rates } & \multicolumn{6}{|c|}{ Panel B: Deposit rates } \\
\hline \multirow[t]{2}{*}{ Intercept } & 0.25 & 0.31 & 0.38 & 0.43 & 0.46 & 0.48 & 0.21 & 0.25 & 0.32 & 0.36 & 0.40 & 0.42 \\
\hline & 2.43 & 3.23 & 3.92 & 4.40 & 4.63 & 4.71 & 2.17 & 2.42 & 3.02 & 3.39 & 3.57 & 3.66 \\
\hline \multicolumn{13}{|l|}{ Regional dummies: } \\
\hline \multirow[t]{2}{*}{ CMA } & 0.23 & 0.35 & 0.33 & 0.31 & 0.28 & 0.24 & 0.20 & 0.21 & 0.19 & 0.15 & 0.12 & 0.10 \\
\hline & 3.46 & 5.75 & 5.41 & 4.94 & 4.41 & 3.71 & 3.23 & 3.22 & 2.80 & 2.25 & 1.73 & 1.36 \\
\hline \multirow[t]{2}{*}{ Botswana } & 0.05 & 0.13 & 0.21 & 0.22 & 0.19 & 0.15 & -0.17 & -0.08 & 0.00 & 0.07 & 0.12 & 0.15 \\
\hline & 0.47 & 1.29 & 1.97 & 2.02 & 1.78 & 1.39 & -1.70 & -0.72 & 0.03 & 0.62 & 1.02 & 1.19 \\
\hline \multicolumn{13}{|l|}{ Time dummies: } \\
\hline \multirow[t]{2}{*}{ 1990-1994 } & 0.01 & 0.03 & 0.05 & 0.08 & 0.13 & 0.16 & 0.20 & 0.22 & 0.31 & 0.40 & 0.46 & 0.51 \\
\hline & 0.06 & 0.19 & 0.33 & 0.54 & 0.84 & 1.01 & 1.33 & 1.36 & 1.89 & 2.36 & 2.62 & 2.77 \\
\hline \multirow[t]{2}{*}{ 1991-1995 } & 0.05 & 0.11 & 0.13 & 0.15 & 0.16 & 0.18 & 0.06 & 0.07 & 0.10 & 0.12 & 0.15 & 0.18 \\
\hline & 0.30 & 0.80 & 0.93 & 1.01 & 1.11 & 1.20 & 0.41 & 0.47 & 0.64 & 0.79 & 0.92 & 1.04 \\
\hline \multirow[t]{2}{*}{ 1992-1996 } & 0.05 & 0.11 & 0.12 & 0.13 & 0.16 & 0.19 & 0.08 & 0.07 & 0.08 & 0.11 & 0.15 & 0.19 \\
\hline & 0.34 & 0.87 & 0.89 & 0.96 & 1.15 & 1.29 & 0.58 & 0.49 & 0.55 & 0.72 & 0.91 & 1.17 \\
\hline \multirow[t]{2}{*}{ 1993-1997 } & 0.12 & 0.15 & 0.15 & 0.14 & 0.14 & 0.15 & 0.05 & 0.11 & 0.17 & 0.23 & 0.28 & 0.31 \\
\hline & 0.85 & 1.14 & 1.11 & 1.00 & 0.98 & 1.03 & 0.34 & 0.77 & 1.12 & 1.48 & 1.74 & 1.89 \\
\hline \multirow[t]{2}{*}{ 1994-1998 } & 0.13 & 0.16 & 0.18 & 0.19 & 0.20 & 0.24 & 0.03 & 0.10 & 0.13 & 0.14 & 0.15 & 0.16 \\
\hline & 0.92 & 1.22 & 1.31 & 1.37 & 1.47 & 1.72 & 0.25 & 0.73 & 0.87 & 0.97 & 0.99 & 1.00 \\
\hline \multirow[t]{2}{*}{ 1995-1999 } & 0.28 & 0.26 & 0.24 & 0.25 & 0.27 & 0.29 & 0.14 & 0.19 & 0.19 & 0.19 & 0.19 & 0.19 \\
\hline & 1.96 & 1.93 & 1.78 & 1.78 & 1.90 & 1.99 & 1.05 & 1.31 & 1.28 & 1.28 & 1.22 & 1.14 \\
\hline \multirow[t]{2}{*}{$1996-2000$} & 0.23 & 0.23 & 0.24 & 0.23 & 0.24 & 0.26 & 0.14 & 0.18 & 0.20 & 0.22 & 0.22 & 0.22 \\
\hline & 1.65 & 1.80 & 1.79 & 1.70 & 1.75 & 1.86 & 1.06 & 1.28 & 1.38 & 1.46 & 1.44 & 1.40 \\
\hline \multirow[t]{2}{*}{$1997-2001$} & 0.08 & 0.13 & 0.13 & 0.12 & 0.12 & 0.13 & 0.03 & 0.08 & 0.13 & 0.16 & 0.17 & 0.17 \\
\hline & 0.58 & 0.97 & 1.00 & 0.88 & 0.86 & 0.93 & 0.25 & 0.60 & 0.89 & 1.07 & 1.11 & 1.06 \\
\hline \multirow[t]{2}{*}{ 1998-2002 } & 0.05 & 0.06 & 0.07 & 0.07 & 0.07 & 0.13 & -0.02 & 0.02 & 0.02 & 0.03 & 0.02 & 0.03 \\
\hline & 0.35 & 0.47 & 0.56 & 0.50 & 0.53 & 0.95 & -0.15 & 0.12 & 0.17 & 0.18 & 0.14 & 0.18 \\
\hline \multirow[t]{2}{*}{ 1999-2003 } & 0.04 & 0.02 & 0.02 & 0.01 & 0.01 & 0.05 & 0.06 & 0.12 & 0.10 & 0.09 & 0.09 & 0.08 \\
\hline & 0.27 & 0.12 & 0.12 & 0.06 & 0.05 & 0.38 & 0.46 & 0.82 & 0.72 & 0.59 & 0.55 & 0.51 \\
\hline \multirow[t]{2}{*}{ 2000-2004 } & -0.04 & -0.02 & -0.01 & -0.01 & -0.02 & -0.02 & -0.02 & -0.01 & 0.00 & 0.01 & 0.01 & 0.00 \\
\hline & -0.29 & -0.18 & -0.06 & -0.10 & -0.14 & -0.14 & -0.15 & -0.04 & 0.00 & 0.04 & 0.03 & 0.01 \\
\hline Adjusted $\mathrm{R}^{2}$ & 0.06 & 0.17 & 0.15 & 0.13 & 0.11 & 0.08 & 0.06 & 0.03 & 0.02 & 0.02 & 0.02 & 0.03 \\
\hline
\end{tabular}

Note: All independent variables are dummies. For each independent variable, the first row reports the estimated coefficient and the second row reports the t-statistics in italics. In Panels A and B, the regressions are based on samples of 147 and 143 observations, respectively. Multipliers for all SADC countries are included. The time period 20012005 serves as a benchmark for the time dummies. 

subject to prior approval of the Minister of Finance. Annual transfers of dividends are subject to approval by BNA. All capital transfers are subject to licensing and control.

Botswana

Lesotho The currency is pegged to a weighted basket of currencies comprising SDR currencies and the South African Rand.

Fixed exchange rate regime (Pegged at par with the South African Rand-CMA).

Independently floating (but IMF notes that the regime operating de facto in the country is different from its de jure regime).

Malawi Managed floating with no pre-determined path for the exchange rate.

Mauritius Managed floating with no preannounced path for exchange rate.

Mozambique Free floating system.

Namibia

Fixed exchange rate system - pegged at par with the South African Rand.

Seychelles

Conventional fixed peg arrangements against a singl currency (but IMF notes that the regime operating de facto in the country is different from its de jure regime).

South Africa Free floating system.

No restrictions on current account transactions. IMF Articles of Agreement with effect from September 1993.

No restrictions are imposed on the exports of goods. The Non-residents and traveler can import any amount of foreign import of goods must be preceded by negotiation of foreign currency against declaration. Only the foreign currency currency with commercial banks. There are no restrictions on previously declared may be exported. Foreigners are allowed the entry of earnings for services. Payments for services to invest in Mozambique. Expatriation of profits and exceeding a value equivalent to US\$5 000.00 must be invested capital are also allowed and regulated by the law. licensed by the central bank

There are no restrictions on current account Namibia acceded to Article VIII of the IMF in 1996.

No capital account restriction within CMA.No restriction on capital from non-residents for equity investment. Outward investments limit for corporate business is $\mathrm{N} \$ 750$ million.

No restrictions on inward investment and disinvestment by non-residents.

There are controls on outward capital investment.

Same as South Africa.

Swaziland Fixed exchange rate system - pegged at par with the South Same as South Africa.

African Rand.

Tanzania Managed floating exchange rate system.

Zambia

Fully market determined.

Zimbabwe
No restrictions on current account transactions in line with IMF's Article VIII since 1996

The is no exchange control on current account.

The capital account is not fully liberalised.

The are no exchange controls on capital account.

. The are exchange controls on capital account.

transaction due to critical shortage of foreign currency -

even though Zimbabwe has ratified the Article VIII of the 
Table A-2: Data description

\begin{tabular}{|c|c|c|c|c|c|c|c|c|c|c|c|c|c|c|}
\hline \multirow[b]{3}{*}{ Country } & \multirow{3}{*}{$\begin{array}{l}\text { Abbre- } \\
\text { viation }\end{array}$} & \multirow{2}{*}{\multicolumn{3}{|c|}{ Member of regional alliance }} & \multirow{3}{*}{ SADC membership status } & \multicolumn{3}{|c|}{ Central bank rate } & \multicolumn{3}{|c|}{ Deposit rate } & \multicolumn{3}{|c|}{ Lending rate } \\
\hline & & & & & & \multirow[b]{2}{*}{ IFS series } & \multicolumn{2}{|c|}{ Sample period } & \multirow[b]{2}{*}{ IFS series } & \multicolumn{2}{|c|}{ Sample period } & \multirow[b]{2}{*}{ IFS series } & \multicolumn{2}{|c|}{ Sample period } \\
\hline & & CMA & SACU & SADC & & & Start & End & & Start & End & & Start & End \\
\hline Angola & ANG & no & no & yes & member from inception & AOI60... & Jan-95 & Dec-05 & AOI60L.. & Jan-95 & Dec-05 & AOI60P.. & Jan-95 & Dec-05 \\
\hline DR Congo & DRC & no & no & yes & member since 1997 & ZAI60... & Jan-90 & Feb-04 & n.a. & & & ZAI60P.. & Jan-94 & May-03 \\
\hline Lesotho & LE & yes & yes & yes & member from inception & LSI60... & Jan-90 & Dec-05 & LSI60L.. & Jan-90 & Dec-05 & LSI60P.. & Jan-90 & Dec-05 \\
\hline Madagascar & MAD & no & no & yes & member since August 2005 & MDI60A.. & Jan-91 & Dec-05 & MDI60L.. & Dec-90 & Dec-05 & MDI60P.. & Dec-90 & Dec-05 \\
\hline Malawi & MAL & no & no & yes & member from inception & MII60... & Jan-90 & Dec-05 & MII60L.. & Jan-90 & Dec-05 & MII60P.. & Jan-90 & Dec-05 \\
\hline Mauritius & MAU & no & no & yes & member since 1995 & MUI60... & Jan-90 & Jun-98 & MUI60L.. & Jan-90 & Dec-05 & MUI60P.. & Jan-90 & Dec-05 \\
\hline Mozambique & MOZ & no & no & yes & member from inception & MZI60... & Jan-94 & Dec-05 & MZI60L.. & Jan-94 & Dec-05 & MZI60P.. & Aug-97 & Dec-05 \\
\hline Namibia & NA & yes & yes & yes & member since 1990 & WAI60... & Sep-91 & Dec-05 & WAI60L.. & Jan-91 & Dec-05 & WAI60P.. & Jan-91 & Dec-05 \\
\hline Seychelles & SEY & no & no & yes & member from 1997 to 2004 & SEI60... & Jan-90 & Dec-05 & SEI60L.. & Jan-90 & Dec-05 & SEI60P.. & Jan-90 & Dec-05 \\
\hline South Africa & SA & yes & yes & yes & member since 1994 & SAI60... & Jan-90 & Dec-05 & SAI60L.. & Jan-90 & Dec-05 & SAI60P.. & Jan-90 & Dec-05 \\
\hline Swaziland & SW & yes & yes & yes & member from inception & SZI60... & Jan-90 & Dec-05 & SZI60L.. & Jan-90 & Dec-05 & SZI60P.. & Jan-90 & Dec-05 \\
\hline Tanzania & TA & no & no & yes & member from inception & TNI60... & May-92 & Dec-05 & TNI60L.. & Sep-94 & Dec-05 & TNI60P.. & May-92 & Dec-05 \\
\hline Zambia & $\mathrm{ZA}$ & no & no & yes & member from inception & ZMI60... & Jan-92 & Dec-05 & ZMI60L.. & Jan-90 & Dec-05 & ZMI60P.. & Jan-90 & Dec-05 \\
\hline
\end{tabular}

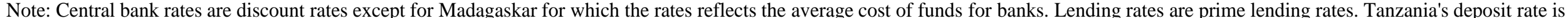

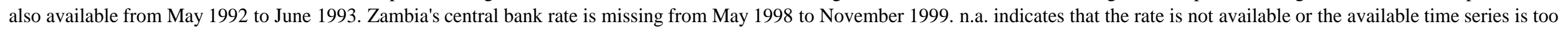
short and or invariant to allow for statistical analysis. 
Table A-3: Multipliers of the pass-through model from national central bank to national retail rates

\begin{tabular}{|c|c|c|c|c|c|c|c|c|c|c|c|}
\hline \multirow[b]{2}{*}{ Country } & \multicolumn{2}{|c|}{ Sample period } & \multicolumn{2}{|c|}{ Cointegration? } & \multirow{2}{*}{$\begin{array}{c}\mathrm{PT} \\
\text { model }\end{array}$} & \multicolumn{6}{|c|}{ Multipliers } \\
\hline & Start & End & Engle/Granger & Kremers & & Impact & $1 \mathrm{mth}$ & 3 mths & 6 mths & 12 mths & Long-run \\
\hline \multicolumn{12}{|c|}{ Panel A: Pass-through from national central bank rate to national deposit rate } \\
\hline Angola & Jan-95 & Dec-99 & no & no & STD & 1.32 & 1.39 & 1.40 & 1.40 & 1.40 & 1.40 \\
\hline Angola & Jan-96 & Dec-00 & no & no & STD & 1.41 & 1.40 & 1.40 & 1.40 & 1.40 & 1.40 \\
\hline Angola & Jan-97 & Dec-01 & yes & yes & SYM & 0.13 & 0.10 & 0.12 & 0.12 & 0.13 & 0.13 \\
\hline Angola & Jan-98 & Dec-02 & yes & yes & SYM & -0.03 & 0.03 & 0.07 & 0.09 & 0.10 & 0.10 \\
\hline Angola & Jan-99 & Dec-03 & yes & yes & SYM & -0.02 & 0.00 & 0.01 & 0.02 & 0.04 & 0.05 \\
\hline Angola & Jan-00 & Dec-04 & yes & no & SYM & 0.08 & 0.09 & 0.16 & 0.23 & 0.31 & 0.40 \\
\hline Angola & Jan-01 & Dec-05 & yes & no & SYM & 0.14 & 0.14 & 0.22 & 0.29 & 0.38 & 0.47 \\
\hline Botswana & Jan-90 & Dec-94 & yes & yes & SYM & 0.60 & 0.53 & 0.72 & 0.84 & 0.92 & 0.94 \\
\hline Botswana & Jan-91 & Dec-95 & yes & no & SYM & 0.13 & 0.16 & 0.24 & 0.34 & 0.46 & 0.59 \\
\hline Botswana & Jan-92 & Dec-96 & yes & yes & SYM & 0.27 & 0.44 & 0.75 & 1.06 & 1.39 & 1.63 \\
\hline Botswana & Jan-93 & Dec-97 & yes & yes & SYM & -0.10 & 0.39 & 0.81 & 1.19 & 1.46 & 1.55 \\
\hline Botswana & Jan-94 & Dec-98 & yes & yes & SYM & -0.28 & 0.32 & 0.56 & 0.65 & 0.67 & 0.67 \\
\hline Botswana & Jan-95 & Dec-99 & yes & yes & SYM & -0.13 & 0.19 & 0.36 & 0.44 & 0.46 & 0.46 \\
\hline Botswana & Jan-96 & Dec-00 & no & yes & SYM & 0.05 & 0.08 & 0.13 & 0.18 & 0.23 & 0.27 \\
\hline Botswana & Jan-97 & Dec-01 & no & yes & SYM & 0.06 & 0.12 & 0.22 & 0.31 & 0.40 & 0.44 \\
\hline Botswana & Jan-98 & Dec-02 & yes & yes & SYM & 0.13 & 0.31 & 0.54 & 0.67 & 0.72 & 0.72 \\
\hline Botswana & Jan-99 & Dec-03 & yes & yes & SYM & 0.07 & 0.16 & 0.29 & 0.42 & 0.54 & 0.60 \\
\hline Botswana & Jan-00 & Dec-04 & yes & yes & SYM & 0.09 & 0.19 & 0.32 & 0.41 & 0.47 & 0.48 \\
\hline Botswana & Jan-01 & Dec-05 & no & no & SYM & 0.25 & 0.27 & 0.34 & 0.41 & 0.49 & 0.58 \\
\hline Lesotho & Jan-90 & Dec-94 & no & no & STD & 0.27 & 0.26 & 0.26 & 0.26 & 0.26 & 0.26 \\
\hline Lesotho & Jan-91 & Dec-95 & no & no & STD & 0.31 & 0.28 & 0.28 & 0.28 & 0.28 & 0.28 \\
\hline Lesotho & Jan-92 & Dec-96 & no & no & STD & 0.35 & 0.31 & 0.32 & 0.32 & 0.32 & 0.32 \\
\hline Lesotho & Jan-93 & Dec-97 & no & no & STD & 0.12 & 0.12 & 0.12 & 0.12 & 0.12 & 0.12 \\
\hline Lesotho & Jan-94 & Dec-98 & no & yes & STD & 0.00 & 0.00 & 0.00 & 0.00 & 0.00 & 0.00 \\
\hline Lesotho & Jan-95 & Dec-99 & no & no & STD & 0.00 & 0.00 & 0.00 & 0.00 & 0.00 & 0.00 \\
\hline Lesotho & Jan-96 & Dec-00 & no & no & STD & 0.00 & 0.00 & 0.00 & 0.00 & 0.00 & 0.00 \\
\hline Lesotho & Jan-97 & Dec-01 & no & no & STD & 0.00 & 0.00 & 0.00 & 0.00 & 0.00 & 0.00 \\
\hline Lesotho & Jan-98 & Dec-02 & no & no & STD & 0.00 & 0.00 & 0.00 & 0.00 & 0.00 & 0.00 \\
\hline Lesotho & Jan-99 & Dec-03 & yes & yes & STD & 0.06 & 0.06 & 0.06 & 0.06 & 0.06 & 0.06 \\
\hline Lesotho & Jan-00 & Dec-04 & no & no & STD & 0.05 & 0.04 & 0.04 & 0.04 & 0.04 & 0.04 \\
\hline Lesotho & Jan-01 & Dec-05 & yes & yes & STD & 0.08 & 0.05 & 0.06 & 0.06 & 0.06 & 0.06 \\
\hline Madagascar & Jan-91 & Dec-95 & yes & yes & SYM & -0.05 & -0.09 & -0.11 & -0.11 & -0.11 & -0.11 \\
\hline Madagascar & Jan-92 & Dec-96 & yes & yes & SYM & -0.06 & -0.09 & -0.09 & -0.08 & -0.08 & -0.08 \\
\hline Madagascar & Jan-93 & Dec-97 & yes & yes & SYM & 0.11 & 0.11 & 0.08 & 0.06 & 0.04 & 0.04 \\
\hline Madagascar & Jan-94 & Dec-98 & no & yes & SYM & 0.19 & 0.22 & 0.24 & 0.27 & 0.29 & 0.31 \\
\hline Madagascar & Jan-95 & Dec-99 & no & yes & SYM & 0.16 & 0.23 & 0.30 & 0.35 & 0.39 & 0.39 \\
\hline Madagascar & Jan-96 & Dec-00 & yes & yes & SYM & 0.24 & 0.32 & 0.40 & 0.46 & 0.49 & 0.49 \\
\hline Madagascar & Jan-97 & Dec-01 & no & yes & SYM & 0.34 & 0.43 & 0.55 & 0.67 & 0.78 & 0.85 \\
\hline Madagascar & Jan-98 & Dec-02 & no & yes & SYM & 0.22 & 0.28 & 0.36 & 0.46 & 0.59 & 0.75 \\
\hline Madagascar & Jan-99 & Dec-03 & yes & yes & SYM & 0.18 & 0.21 & 0.25 & 0.29 & 0.34 & 0.37 \\
\hline Madagascar & Jan-00 & Dec-04 & yes & yes & SYM & 0.05 & 0.19 & 0.34 & 0.41 & 0.44 & 0.44 \\
\hline Madagascar & Jan-01 & Dec-05 & yes & yes & SYM & 0.07 & 0.17 & 0.31 & 0.43 & 0.50 & 0.52 \\
\hline Malawi & Jan-90 & Dec-94 & yes & yes & SYM & 0.32 & 0.45 & 0.65 & 0.77 & 0.83 & 0.83 \\
\hline Malawi & Jan-91 & Dec-95 & yes & yes & SYM & 0.52 & 0.61 & 0.68 & 0.71 & 0.72 & 0.72 \\
\hline Malawi & Jan-92 & Dec-96 & no & no & STD & 0.58 & 0.58 & 0.58 & 0.58 & 0.58 & 0.58 \\
\hline Malawi & Jan-93 & Dec-97 & no & no & STD & 0.52 & 0.52 & 0.52 & 0.52 & 0.52 & 0.52 \\
\hline Malawi & Jan-94 & Dec-98 & no & no & STD & 0.56 & 0.59 & 0.59 & 0.59 & 0.59 & 0.59 \\
\hline Malawi & Jan-95 & Dec-99 & yes & yes & SYM & 0.90 & 1.00 & 1.04 & 1.05 & 1.06 & 1.06 \\
\hline Malawi & Jan-96 & Dec-00 & yes & yes & SYM & 0.90 & 1.02 & 1.02 & 1.01 & 1.01 & 1.01 \\
\hline Malawi & Jan-97 & Dec-01 & yes & yes & SYM & 0.29 & 0.44 & 0.62 & 0.76 & 0.83 & 0.84 \\
\hline Malawi & Jan-98 & Dec-02 & yes & yes & SYM & 0.30 & 0.46 & 0.62 & 0.69 & 0.72 & 0.72 \\
\hline Malawi & Jan-99 & Dec-03 & yes & yes & SYM & 0.24 & 0.35 & 0.48 & 0.56 & 0.60 & 0.60 \\
\hline Malawi & Jan-00 & Dec-04 & yes & yes & SYM & 0.32 & 0.45 & 0.62 & 0.73 & 0.79 & 0.80 \\
\hline Malawi & Jan-01 & Dec-05 & yes & yes & SYM & 0.33 & 0.52 & 0.70 & 0.77 & 0.79 & 0.79 \\
\hline Mauritius & Jan-90 & Dec-94 & yes & yes & SYM & 0.70 & 0.68 & 0.79 & 0.87 & 0.91 & 0.92 \\
\hline Mauritius & Jan-91 & Dec-95 & yes & yes & SYM & 0.68 & 0.68 & 0.78 & 0.85 & 0.89 & 0.90 \\
\hline Mauritius & Jan-92 & Dec-96 & yes & yes & SYM & 0.67 & 0.62 & 0.71 & 0.77 & 0.82 & 0.84 \\
\hline Mauritius & Jan-93 & Dec-97 & yes & no & SYM & 0.23 & 0.24 & 0.32 & 0.41 & 0.54 & 0.72 \\
\hline Mauritius & Jan-94 & Jun-98 & yes & no & SYM & 0.23 & 0.25 & 0.33 & 0.42 & 0.54 & 0.68 \\
\hline Mozambique & Jan-94 & Dec-98 & no & yes & SYM & 0.24 & 0.29 & 0.33 & 0.36 & 0.39 & 0.40 \\
\hline Mozambique & Jan-95 & Dec-99 & no & yes & SYM & -0.20 & -0.11 & 0.05 & 0.20 & 0.34 & 0.39 \\
\hline Mozambique & Jan-96 & Dec-00 & no & yes & SYM & -0.36 & -0.29 & -0.14 & 0.00 & 0.15 & 0.23 \\
\hline
\end{tabular}


Table A-3: Multipliers of the pass-through model from national central bank to national retail rates

\begin{tabular}{|c|c|c|c|c|c|c|c|c|c|c|c|}
\hline \multirow[b]{2}{*}{ Country } & \multicolumn{2}{|c|}{ Sample period } & \multicolumn{2}{|c|}{ Cointegration? } & \multirow{2}{*}{$\begin{array}{c}\mathrm{PT} \\
\text { model }\end{array}$} & \multicolumn{6}{|c|}{ Multipliers } \\
\hline & Start & End & Engle/Granger & Kremers & & Impact & $1 \mathrm{mth}$ & 3 mths & 6 mths & 12 mths & Long-run \\
\hline Mozambique & Jan-97 & Dec-01 & yes & yes & SYM & 0.30 & 0.63 & 1.08 & 1.38 & 1.53 & 1.56 \\
\hline Mozambique & Jan-98 & Dec-02 & no & no & STD & -0.08 & -0.09 & -0.09 & -0.09 & -0.09 & -0.09 \\
\hline Mozambique & Jan-99 & Dec-03 & no & no & STD & 0.00 & 0.00 & 0.00 & 0.00 & 0.00 & 0.00 \\
\hline Mozambique & Jan-00 & Dec-04 & no & no & STD & 0.00 & 0.00 & 0.00 & 0.00 & 0.00 & 0.00 \\
\hline Mozambique & Jan-01 & Dec-05 & no & no & STD & 0.00 & 0.00 & 0.00 & 0.00 & 0.00 & 0.00 \\
\hline Namibia & Jan-91 & Dec-95 & yes & yes & SYM & 0.05 & 0.36 & 0.61 & 0.62 & 0.62 & 0.62 \\
\hline Namibia & Jan-92 & Dec-96 & yes & yes & SYM & 0.10 & 0.22 & 0.40 & 0.57 & 0.74 & 0.83 \\
\hline Namibia & Jan-93 & Dec-97 & yes & yes & SYM & 0.03 & 0.32 & 0.67 & 0.93 & 1.10 & 1.14 \\
\hline Namibia & Jan-94 & Dec-98 & yes & yes & SYM & 0.23 & 0.40 & 0.61 & 0.77 & 0.85 & 0.87 \\
\hline Namibia & Jan-95 & Dec-99 & no & yes & SYM & 0.26 & 0.38 & 0.49 & 0.54 & 0.57 & 0.57 \\
\hline Namibia & Jan-96 & Dec-00 & yes & yes & SYM & 0.29 & 0.47 & 0.64 & 0.72 & 0.74 & 0.74 \\
\hline Namibia & Jan-97 & Dec-01 & no & yes & SYM & 0.28 & 0.45 & 0.63 & 0.73 & 0.77 & 0.77 \\
\hline Namibia & Jan-98 & Dec-02 & no & yes & SYM & 0.26 & 0.44 & 0.64 & 0.73 & 0.76 & 0.76 \\
\hline Namibia & Jan-99 & Dec-03 & no & yes & SYM & 0.15 & 0.30 & 0.51 & 0.67 & 0.76 & 0.78 \\
\hline Namibia & Jan-00 & Dec-04 & no & yes & SYM & 0.02 & 0.09 & 0.21 & 0.32 & 0.40 & 0.42 \\
\hline Namibia & Jan-01 & Dec-05 & no & yes & SYM & 0.01 & 0.10 & 0.24 & 0.34 & 0.41 & 0.43 \\
\hline South Africa & Jan-90 & Dec-94 & no & no & SYM & 0.66 & 0.88 & 1.04 & 1.14 & 1.26 & 1.35 \\
\hline South Africa & Jan-91 & Dec-95 & yes & no & SYM & 0.79 & 0.88 & 0.98 & 1.07 & 1.17 & 1.23 \\
\hline South Africa & Jan-92 & Dec-96 & yes & yes & SYM & 0.80 & 0.94 & 1.01 & 1.04 & 1.05 & 1.06 \\
\hline South Africa & Jan-93 & Dec-97 & yes & yes & SYM & 0.80 & 0.93 & 0.99 & 1.01 & 1.01 & 1.01 \\
\hline South Africa & Jan-94 & Dec-98 & yes & yes & SYM & 0.57 & 0.80 & 0.88 & 0.89 & 0.89 & 0.89 \\
\hline South Africa & Jan-95 & Dec-99 & yes & yes & SYM & 0.76 & 0.87 & 0.90 & 0.90 & 0.90 & 0.90 \\
\hline South Africa & Jan-96 & Dec-00 & yes & yes & SYM & 0.73 & 0.91 & 1.02 & 1.05 & 1.06 & 1.06 \\
\hline South Africa & Jan-97 & Dec-01 & yes & yes & SYM & 0.62 & 0.86 & 0.97 & 0.98 & 0.98 & 0.98 \\
\hline South Africa & Jan-98 & Dec-02 & yes & yes & SYM & 0.65 & 0.88 & 0.93 & 0.92 & 0.92 & 0.92 \\
\hline South Africa & Jan-99 & Dec-03 & yes & yes & SYM & 0.50 & 0.71 & 0.81 & 0.81 & 0.80 & 0.80 \\
\hline South Africa & Jan-00 & Dec-04 & no & yes & SYM & 0.52 & 0.73 & 0.82 & 0.81 & 0.81 & 0.81 \\
\hline South Africa & Jan-01 & Dec-05 & yes & yes & SYM & 0.56 & 0.81 & 0.91 & 0.87 & 0.86 & 0.86 \\
\hline Seychelles & Jan-90 & Dec-94 & yes & no & SYM & 0.01 & 0.11 & 0.28 & 0.48 & 0.71 & 0.94 \\
\hline Seychelles & Jan-91 & Dec-95 & no & no & SYM & 0.00 & 0.05 & 0.13 & 0.22 & 0.35 & 0.50 \\
\hline Seychelles & Jan-92 & Dec-96 & no & yes & SYM & -0.12 & -0.14 & -0.13 & -0.13 & -0.12 & -0.12 \\
\hline Seychelles & Jan-93 & Dec-97 & no & yes & SYM & -0.14 & -0.15 & -0.11 & -0.08 & -0.05 & -0.05 \\
\hline Seychelles & Jan-94 & Dec-98 & no & yes & SYM & 0.27 & 0.32 & 0.34 & 0.36 & 0.38 & 0.39 \\
\hline Seychelles & Jan-95 & Dec-99 & no & yes & SYM & 0.28 & 0.37 & 0.46 & 0.53 & 0.58 & 0.60 \\
\hline Seychelles & Jan-96 & Dec-00 & yes & yes & SYM & 0.32 & 0.46 & 0.61 & 0.69 & 0.72 & 0.72 \\
\hline Seychelles & Jan-97 & Dec-01 & yes & yes & SYM & 0.37 & 0.50 & 0.63 & 0.70 & 0.73 & 0.74 \\
\hline Seychelles & Jan-98 & Dec-02 & yes & yes & SYM & 0.37 & 0.51 & 0.64 & 0.69 & 0.71 & 0.71 \\
\hline Seychelles & Jan-99 & Dec-03 & yes & yes & SYM & 1.18 & 1.83 & 1.86 & 1.71 & 1.73 & 1.73 \\
\hline Seychelles & Jan-00 & Dec-04 & yes & yes & SYM & 0.61 & 0.69 & 0.66 & 0.63 & 0.62 & 0.62 \\
\hline Seychelles & Jan-01 & Dec-05 & yes & yes & SYM & 0.53 & 0.53 & 0.55 & 0.56 & 0.57 & 0.57 \\
\hline Swaziland & Jan-90 & Dec-94 & yes & yes & SYM & 1.12 & 1.20 & 1.30 & 1.41 & 1.54 & 1.65 \\
\hline Swaziland & Jan-91 & Dec-95 & no & no & STD & 0.86 & 0.91 & 0.91 & 0.91 & 0.91 & 0.91 \\
\hline Swaziland & Jan-92 & Dec-96 & no & no & STD & 0.88 & 0.90 & 0.90 & 0.90 & 0.90 & 0.90 \\
\hline Swaziland & Jan-93 & Dec-97 & no & yes & SYM & 0.80 & 0.78 & 0.79 & 0.79 & 0.80 & 0.80 \\
\hline Swaziland & Jan-94 & Dec-98 & no & yes & SYM & 0.90 & 0.90 & 0.89 & 0.88 & 0.87 & 0.86 \\
\hline Swaziland & Jan-95 & Dec-99 & yes & yes & SYM & 0.96 & 0.99 & 0.99 & 0.99 & 0.99 & 0.99 \\
\hline Swaziland & Jan-96 & Dec-00 & yes & yes & SYM & 1.02 & 1.03 & 0.99 & 0.99 & 0.99 & 0.99 \\
\hline Swaziland & Jan-97 & Dec-01 & yes & yes & SYM & 0.98 & 0.99 & 0.97 & 0.96 & 0.95 & 0.95 \\
\hline Swaziland & Jan-98 & Dec-02 & yes & yes & SYM & 0.96 & 0.99 & 0.96 & 0.95 & 0.94 & 0.94 \\
\hline Swaziland & Jan-99 & Dec-03 & no & yes & SYM & 0.83 & 0.85 & 0.86 & 0.87 & 0.88 & 0.89 \\
\hline Swaziland & Jan-00 & Dec-04 & no & no & SYM & 0.76 & 0.75 & 0.75 & 0.75 & 0.76 & 0.77 \\
\hline Swaziland & Jan-01 & Dec-05 & no & yes & SYM & 0.77 & 0.74 & 0.75 & 0.76 & 0.78 & 0.78 \\
\hline Tanzania & Jan-94 & Dec-98 & yes & yes & SYM & 0.10 & 0.19 & 0.30 & 0.39 & 0.44 & 0.45 \\
\hline Tanzania & Jan-95 & Dec-99 & yes & yes & SYM & 0.12 & 0.22 & 0.34 & 0.43 & 0.47 & 0.48 \\
\hline Tanzania & Jan-96 & Dec-00 & yes & yes & SYM & 0.21 & 0.36 & 0.51 & 0.57 & 0.59 & 0.59 \\
\hline Tanzania & Jan-97 & Dec-01 & yes & yes & SYM & 0.09 & 0.16 & 0.23 & 0.27 & 0.29 & 0.30 \\
\hline Tanzania & Jan-98 & Dec-02 & yes & yes & SYM & 0.08 & 0.14 & 0.23 & 0.31 & 0.37 & 0.40 \\
\hline Tanzania & Jan-99 & Dec-03 & no & yes & SYM & 0.11 & 0.14 & 0.23 & 0.32 & 0.41 & 0.47 \\
\hline Tanzania & Jan-00 & Dec-04 & no & yes & SYM & 0.10 & 0.14 & 0.23 & 0.31 & 0.38 & 0.42 \\
\hline Tanzania & Jan-01 & Dec-05 & yes & yes & SYM & 0.18 & 0.16 & 0.15 & 0.14 & 0.13 & 0.13 \\
\hline Zambia & Jan-92 & Dec-96 & yes & yes & SYM & 0.40 & 0.60 & 0.76 & 0.81 & 0.83 & 0.83 \\
\hline Zambia & Jan-93 & Dec-97 & yes & yes & SYM & 0.34 & 0.54 & 0.72 & 0.77 & 0.78 & 0.78 \\
\hline Zambia & Jan-94 & Apr-98 & no & yes & SYM & 0.25 & 0.41 & 0.58 & 0.67 & 0.69 & 0.69 \\
\hline
\end{tabular}


Table A-3: Multipliers of the pass-through model from national central bank to national retail rates

\begin{tabular}{|c|c|c|c|c|c|c|c|c|c|c|c|}
\hline \multirow[b]{2}{*}{ Country } & \multicolumn{2}{|c|}{ Sample period } & \multicolumn{2}{|c|}{ Cointegration? } & \multirow{2}{*}{$\begin{array}{c}\text { PT } \\
\text { model }\end{array}$} & \multicolumn{6}{|c|}{ Multipliers } \\
\hline & Start & End & Engle/Granger & Kremers & & Impact & $1 \mathrm{mth}$ & 3 mths & 6 mths & 12 mths & Long-run \\
\hline Zambia & Jan-95 & Dec-99 & n.a. & n.a. & n.a. & n.a. & n.a. & n.a. & n.a. & n.a. & n.a. \\
\hline Zambia & Jan-96 & Dec-00 & no & yes & SYM & 0.03 & 0.14 & 0.34 & 0.54 & 0.71 & 0.79 \\
\hline Zambia & Jan-97 & Dec-01 & no & yes & SYM & 0.01 & 0.05 & 0.12 & 0.20 & 0.27 & 0.31 \\
\hline Zambia & Jan-98 & Dec-02 & no & yes & SYM & 0.12 & 0.15 & 0.19 & 0.24 & 0.30 & 0.41 \\
\hline Zambia & Jan-99 & Dec-03 & no & yes & SYM & 0.07 & 0.10 & 0.13 & 0.14 & 0.16 & 0.17 \\
\hline Zambia & Jan-00 & Dec-04 & no & yes & SYM & 0.05 & 0.12 & 0.27 & 0.40 & 0.44 & 0.43 \\
\hline Zambia & Jan-01 & Dec-05 & no & yes & SYM & 0.05 & 0.13 & 0.27 & 0.40 & 0.49 & 0.50 \\
\hline Zimbabwe & Jan-90 & Dec-94 & yes & yes & SYM & -0.03 & 0.16 & 0.56 & 0.87 & 1.00 & 1.01 \\
\hline Zimbabwe & Jan-91 & Dec-95 & yes & yes & SYM & -0.04 & 0.10 & 0.40 & 0.68 & 0.88 & 0.94 \\
\hline Zimbabwe & Jan-92 & Dec-96 & no & no & SYM & -0.13 & -0.12 & -0.04 & 0.07 & 0.24 & 0.59 \\
\hline Zimbabwe & Jan-93 & Dec-97 & no & yes & SYM & 0.67 & 0.91 & 1.20 & 1.49 & 1.82 & 2.06 \\
\hline Zimbabwe & Jan-94 & Dec-98 & yes & yes & SYM & 0.46 & 0.62 & 0.83 & 1.04 & 1.24 & 1.36 \\
\hline Zimbabwe & Jan-95 & Dec-99 & no & yes & SYM & 0.34 & 0.43 & 0.49 & 0.54 & 0.59 & 0.62 \\
\hline Zimbabwe & Jan-96 & Dec-00 & yes & yes & SYM & 0.26 & 0.40 & 0.54 & 0.63 & 0.67 & 0.68 \\
\hline Zimbabwe & Jan-97 & Dec-01 & no & no & STD & 0.23 & 0.33 & 0.39 & 0.40 & 0.40 & 0.40 \\
\hline Zimbabwe & Jan-98 & Dec-02 & no & no & STD & 0.06 & 0.09 & 0.10 & 0.10 & 0.10 & 0.10 \\
\hline Zimbabwe & Jan-99 & Dec-03 & no & yes & SYM & 0.70 & 0.75 & 0.72 & 0.68 & 0.65 & 0.62 \\
\hline Zimbabwe & Jan-00 & Dec-04 & yes & yes & SYM & 0.38 & 0.41 & 0.45 & 0.46 & 0.46 & 0.46 \\
\hline Zimbabwe & Jan-01 & Dec-05 & yes & yes & SYM & 0.32 & 0.34 & 0.38 & 0.39 & 0.39 & 0.39 \\
\hline \multicolumn{12}{|c|}{ Panel B: Pass-through from national central bank rate to national loan rate } \\
\hline Angola & Jan-95 & Dec-99 & yes & yes & SYM & 1.89 & 1.81 & 1.93 & 1.99 & 2.02 & 2.03 \\
\hline Angola & Jan-96 & Dec-00 & yes & yes & SYM & 2.04 & 1.71 & 1.70 & 1.66 & 1.63 & 1.62 \\
\hline Angola & Jan-97 & Dec-01 & yes & yes & SYM & 0.44 & 0.58 & 0.63 & 0.63 & 0.63 & 0.63 \\
\hline Angola & Jan-98 & Dec-02 & yes & yes & SYM & 0.26 & 0.41 & 0.53 & 0.56 & 0.56 & 0.56 \\
\hline Angola & Jan-99 & Dec-03 & yes & yes & SYM & 0.22 & 0.34 & 0.42 & 0.44 & 0.45 & 0.45 \\
\hline Angola & Jan-00 & Dec-04 & yes & yes & SYM & 0.08 & 0.18 & 0.25 & 0.27 & 0.27 & 0.27 \\
\hline Angola & Jan-01 & Dec-05 & yes & yes & SYM & 0.12 & 0.26 & 0.39 & 0.43 & 0.44 & 0.44 \\
\hline Botswana & Jan-90 & Dec-94 & yes & yes & SYM & 0.67 & 0.76 & 0.85 & 0.92 & 0.95 & 0.95 \\
\hline Botswana & Jan-91 & Dec-95 & yes & yes & SYM & 0.54 & 0.76 & 0.99 & 1.10 & 1.14 & 1.14 \\
\hline Botswana & Jan-92 & Dec-96 & yes & yes & SYM & 0.45 & 0.39 & 0.45 & 0.49 & 0.51 & 0.52 \\
\hline Botswana & Jan-93 & Dec-97 & yes & yes & SYM & 0.39 & 0.33 & 0.36 & 0.37 & 0.38 & 0.38 \\
\hline Botswana & Jan-94 & Dec-98 & yes & yes & SYM & 0.02 & 0.11 & 0.24 & 0.33 & 0.37 & 0.38 \\
\hline Botswana & Jan-95 & Dec-99 & no & yes & SYM & 0.30 & 0.37 & 0.45 & 0.51 & 0.55 & 0.56 \\
\hline Botswana & Jan-96 & Dec-00 & yes & yes & SYM & 0.23 & 0.52 & 0.81 & 0.91 & 0.93 & 0.93 \\
\hline Botswana & Jan-97 & Dec-01 & yes & yes & SYM & 0.21 & 0.51 & 0.81 & 0.93 & 0.95 & 0.95 \\
\hline Botswana & Jan-98 & Dec-02 & yes & yes & SYM & 0.25 & 0.59 & 0.89 & 0.96 & 0.97 & 0.97 \\
\hline Botswana & Jan-99 & Dec-03 & yes & yes & SYM & 0.31 & 0.60 & 0.83 & 0.87 & 0.87 & 0.87 \\
\hline Botswana & Jan-00 & Dec-04 & yes & yes & SYM & 0.25 & 0.50 & 0.72 & 0.76 & 0.76 & 0.76 \\
\hline Botswana & Jan-01 & Dec-05 & yes & yes & SYM & 0.99 & 1.02 & 0.99 & 0.99 & 0.99 & 0.99 \\
\hline DR Congo & Jan-94 & Dec-98 & no & no & SYM & 0.52 & 0.63 & 0.70 & 0.78 & 0.91 & 1.29 \\
\hline DR Congo & Jan-95 & Dec-99 & yes & yes & SYM & 0.48 & 0.65 & 0.87 & 1.05 & 1.18 & 1.21 \\
\hline DR Congo & Jan-96 & Dec-00 & yes & yes & SYM & 0.48 & 0.84 & 1.08 & 1.11 & 1.11 & 1.11 \\
\hline DR Congo & Jan-97 & Dec-01 & yes & yes & SYM & 0.56 & 0.94 & 1.12 & 1.11 & 1.11 & 1.11 \\
\hline DR Congo & Jan-98 & Dec-02 & yes & yes & SYM & 0.76 & 1.01 & 1.16 & 1.20 & 1.20 & 1.20 \\
\hline DR Congo & Jan-99 & May-03 & yes & yes & SYM & 0.75 & 0.99 & 1.14 & 1.17 & 1.18 & 1.18 \\
\hline Lesotho & Jan-90 & Dec-94 & yes & yes & SYM & 0.64 & 0.64 & 0.90 & 1.07 & 1.17 & 1.19 \\
\hline Lesotho & Jan-91 & Dec-95 & yes & yes & SYM & 0.88 & 0.87 & 1.05 & 1.10 & 1.11 & 1.11 \\
\hline Lesotho & Jan-92 & Dec-96 & yes & yes & SYM & 0.94 & 0.90 & 1.00 & 1.02 & 1.02 & 1.02 \\
\hline Lesotho & Jan-93 & Dec-97 & yes & yes & SYM & 1.07 & 1.02 & 1.11 & 1.12 & 1.12 & 1.12 \\
\hline Lesotho & Jan-94 & Dec-98 & yes & yes & SYM & 0.80 & 0.88 & 0.99 & 1.07 & 1.11 & 1.12 \\
\hline Lesotho & Jan-95 & Dec-99 & yes & no & SYM & 0.75 & 0.73 & 0.76 & 0.79 & 0.82 & 0.84 \\
\hline Lesotho & Jan-96 & Dec-00 & no & yes & SYM & 0.59 & 0.60 & 0.61 & 0.61 & 0.61 & 0.62 \\
\hline Lesotho & Jan-97 & Dec-01 & no & yes & SYM & 0.56 & 0.55 & 0.55 & 0.56 & 0.57 & 0.58 \\
\hline Lesotho & Jan-98 & Dec-02 & no & yes & SYM & 0.51 & 0.51 & 0.52 & 0.52 & 0.52 & 0.53 \\
\hline Lesotho & Jan-99 & Dec-03 & no & no & STD & 0.13 & 0.13 & 0.13 & 0.13 & 0.13 & 0.13 \\
\hline Lesotho & Jan-00 & Dec-04 & no & no & STD & 0.09 & 0.11 & 0.12 & 0.12 & 0.12 & 0.12 \\
\hline Lesotho & Jan-01 & Dec-05 & no & no & STD & 0.20 & 0.24 & 0.26 & 0.26 & 0.26 & 0.26 \\
\hline Madagascar & Jan-91 & Dec-95 & yes & yes & SYM & 0.34 & 0.32 & 0.39 & 0.44 & 0.48 & 0.49 \\
\hline Madagascar & Jan-92 & Dec-96 & yes & yes & SYM & 0.27 & 0.25 & 0.35 & 0.43 & 0.50 & 0.53 \\
\hline Madagascar & Jan-93 & Dec-97 & no & yes & SYM & 0.22 & 0.20 & 0.27 & 0.33 & 0.39 & 0.43 \\
\hline Madagascar & Jan-94 & Dec-98 & yes & yes & SYM & 0.18 & 0.21 & 0.28 & 0.34 & 0.37 & 0.38 \\
\hline Madagascar & Jan-95 & Dec-99 & yes & yes & SYM & 0.13 & 0.19 & 0.28 & 0.35 & 0.38 & 0.39 \\
\hline Madagascar & Jan-96 & Dec-00 & no & yes & SYM & 0.15 & 0.18 & 0.25 & 0.32 & 0.40 & 0.47 \\
\hline
\end{tabular}


Table A-3: Multipliers of the pass-through model from national central bank to national retail rates

\begin{tabular}{|c|c|c|c|c|c|c|c|c|c|c|c|}
\hline \multirow[b]{2}{*}{ Country } & \multicolumn{2}{|c|}{ Sample period } & \multicolumn{2}{|c|}{ Cointegration? } & \multirow{2}{*}{$\begin{array}{c}\mathrm{PT} \\
\text { model }\end{array}$} & \multicolumn{6}{|c|}{ Multipliers } \\
\hline & Start & End & Engle/Granger & Kremers & & Impact & $1 \mathrm{mth}$ & 3 mths & 6 mths & 12 mths & Long-run \\
\hline Madagascar & Jan-97 & Dec-01 & no & yes & SYM & 0.09 & 0.10 & 0.12 & 0.14 & 0.18 & 0.24 \\
\hline Madagascar & Jan-98 & Dec-02 & no & no & SYM & 0.02 & 0.03 & 0.04 & 0.05 & 0.08 & 0.19 \\
\hline Madagascar & Jan-99 & Dec-03 & yes & yes & SYM & 0.08 & 0.12 & 0.18 & 0.25 & 0.31 & 0.36 \\
\hline Madagascar & Jan-00 & Dec-04 & no & yes & SYM & 0.04 & 0.07 & 0.11 & 0.15 & 0.20 & 0.23 \\
\hline Madagascar & Jan-01 & Dec-05 & no & yes & SYM & 0.04 & 0.06 & 0.08 & 0.11 & 0.13 & 0.14 \\
\hline Malawi & Jan-90 & Dec-94 & yes & yes & SYM & 0.04 & 0.15 & 0.32 & 0.48 & 0.63 & 0.69 \\
\hline Malawi & Jan-91 & Dec-95 & yes & yes & SYM & 0.54 & 0.67 & 0.77 & 0.82 & 0.83 & 0.84 \\
\hline Malawi & Jan-92 & Dec-96 & yes & yes & SYM & 0.58 & 0.70 & 0.78 & 0.82 & 0.84 & 0.84 \\
\hline Malawi & Jan-93 & Dec-97 & yes & yes & SYM & 0.64 & 0.74 & 0.79 & 0.82 & 0.83 & 0.83 \\
\hline Malawi & Jan-94 & Dec-98 & yes & yes & SYM & 0.74 & 0.86 & 0.89 & 0.89 & 0.89 & 0.89 \\
\hline Malawi & Jan-95 & Dec-99 & yes & yes & SYM & 1.05 & 1.10 & 1.05 & 1.01 & 1.00 & 1.00 \\
\hline Malawi & Jan-96 & Dec-00 & no & yes & SYM & 0.90 & 1.00 & 1.02 & 1.04 & 1.05 & 1.05 \\
\hline Malawi & Jan-97 & Dec-01 & yes & yes & SYM & 0.33 & 0.47 & 0.67 & 0.82 & 0.91 & 0.94 \\
\hline Malawi & Jan-98 & Dec-02 & yes & yes & SYM & 0.34 & 0.50 & 0.67 & 0.76 & 0.79 & 0.79 \\
\hline Malawi & Jan-99 & Dec-03 & yes & yes & SYM & 0.29 & 0.42 & 0.49 & 0.51 & 0.51 & 0.51 \\
\hline Malawi & Jan-00 & Dec-04 & yes & yes & SYM & 0.35 & 0.48 & 0.63 & 0.70 & 0.72 & 0.72 \\
\hline Malawi & Jan-01 & Dec-05 & yes & yes & SYM & 0.33 & 0.48 & 0.65 & 0.73 & 0.76 & 0.76 \\
\hline Mauritius & Jan-90 & Dec-94 & no & no & STD & 0.31 & 0.34 & 0.35 & 0.35 & 0.35 & 0.35 \\
\hline Mauritius & Jan-91 & Dec-95 & no & no & STD & 0.35 & 0.38 & 0.38 & 0.38 & 0.38 & 0.38 \\
\hline Mauritius & Jan-92 & Dec-96 & no & yes & SYM & 0.41 & 0.48 & 0.58 & 0.69 & 0.82 & 0.93 \\
\hline Mauritius & Jan-93 & Dec-97 & yes & yes & SYM & 0.62 & 0.78 & 0.89 & 0.93 & 0.94 & 0.94 \\
\hline Mauritius & Jan-94 & Jun-98 & yes & yes & SYM & 0.57 & 0.71 & 0.77 & 0.78 & 0.79 & 0.79 \\
\hline Mozambique & Jan-98 & Dec-02 & yes & yes & SYM & -0.01 & 0.00 & 0.02 & 0.05 & 0.10 & 0.63 \\
\hline Mozambique & Jan-99 & Dec-03 & no & no & STD & 0.00 & 0.00 & 0.00 & 0.00 & 0.00 & 0.00 \\
\hline Mozambique & Jan-00 & Dec-04 & no & no & STD & 0.00 & 0.00 & 0.00 & 0.00 & 0.00 & 0.00 \\
\hline Mozambique & Jan-01 & Dec-05 & no & no & STD & 0.00 & 0.00 & 0.00 & 0.00 & 0.00 & 0.00 \\
\hline Namibia & Jan-91 & Dec-95 & no & no & SYM & -0.04 & 0.53 & 0.75 & 0.81 & 0.82 & 0.82 \\
\hline Namibia & Jan-92 & Dec-96 & yes & yes & SYM & -0.20 & 0.55 & 0.71 & 0.76 & 0.77 & 0.77 \\
\hline Namibia & Jan-93 & Dec-97 & yes & yes & SYM & 0.02 & 0.48 & 0.73 & 0.81 & 0.82 & 0.82 \\
\hline Namibia & Jan-94 & Dec-98 & yes & yes & SYM & 0.24 & 0.51 & 0.72 & 0.81 & 0.84 & 0.84 \\
\hline Namibia & Jan-95 & Dec-99 & yes & yes & SYM & 0.21 & 0.35 & 0.48 & 0.55 & 0.57 & 0.57 \\
\hline Namibia & Jan-96 & Dec-00 & yes & yes & SYM & 0.17 & 0.46 & 0.62 & 0.67 & 0.68 & 0.68 \\
\hline Namibia & Jan-97 & Dec-01 & yes & yes & SYM & 0.22 & 0.55 & 0.71 & 0.76 & 0.77 & 0.77 \\
\hline Namibia & Jan-98 & Dec-02 & yes & yes & SYM & 0.15 & 0.43 & 0.65 & 0.77 & 0.82 & 0.83 \\
\hline Namibia & Jan-99 & Dec-03 & yes & yes & SYM & 0.11 & 0.41 & 0.66 & 0.82 & 0.88 & 0.89 \\
\hline Namibia & Jan-00 & Dec-04 & yes & yes & SYM & -0.11 & 0.33 & 0.57 & 0.71 & 0.76 & 0.76 \\
\hline Namibia & Jan-01 & Dec-05 & yes & yes & SYM & -0.13 & 0.38 & 0.62 & 0.75 & 0.80 & 0.80 \\
\hline South Africa & Jan-90 & Dec-94 & yes & yes & SYM & 0.32 & 0.91 & 0.97 & 0.96 & 0.96 & 0.96 \\
\hline South Africa & Jan-91 & Dec-95 & yes & yes & SYM & 0.44 & 1.01 & 0.99 & 0.98 & 0.98 & 0.98 \\
\hline South Africa & Jan-92 & Dec-96 & yes & yes & SYM & 0.62 & 1.14 & 1.07 & 1.05 & 1.05 & 1.05 \\
\hline South Africa & Jan-93 & Dec-97 & yes & yes & SYM & 0.81 & 1.10 & 1.04 & 1.01 & 1.02 & 1.02 \\
\hline South Africa & Jan-94 & Dec-98 & yes & yes & SYM & 0.45 & 0.96 & 1.01 & 0.98 & 0.98 & 0.98 \\
\hline South Africa & Jan-95 & Dec-99 & yes & yes & SYM & 0.42 & 0.98 & 0.98 & 0.95 & 0.95 & 0.95 \\
\hline South Africa & Jan-96 & Dec-00 & yes & yes & SYM & 0.49 & 0.96 & 1.06 & 1.03 & 1.04 & 1.04 \\
\hline South Africa & Jan-97 & Dec-01 & yes & yes & SYM & 0.47 & 0.94 & 1.04 & 1.03 & 1.03 & 1.03 \\
\hline South Africa & Jan-98 & Dec-02 & yes & yes & SYM & 0.59 & 0.91 & 1.02 & 1.01 & 1.01 & 1.01 \\
\hline South Africa & Jan-99 & Dec-03 & yes & yes & SYM & 0.85 & 0.86 & 0.93 & 0.98 & 1.01 & 1.02 \\
\hline South Africa & Jan-00 & Dec-04 & no & yes & SYM & 0.87 & 0.91 & 0.92 & 0.92 & 0.92 & 0.93 \\
\hline South Africa & Jan-01 & Dec-05 & no & no & SYM & 0.89 & 0.93 & 0.94 & 0.95 & 0.95 & 0.96 \\
\hline Seychelles & Jan-90 & Dec-94 & no & yes & SYM & -0.03 & -0.05 & -0.07 & -0.09 & -0.12 & -0.14 \\
\hline Seychelles & Jan-91 & Dec-95 & no & yes & SYM & -0.02 & -0.04 & -0.08 & -0.11 & -0.15 & -0.17 \\
\hline Seychelles & Jan-92 & Dec-96 & yes & yes & SYM & -0.07 & -0.08 & -0.13 & -0.18 & -0.23 & -0.30 \\
\hline Seychelles & Jan-93 & Dec-97 & no & no & SYM & -0.09 & -0.04 & 0.05 & 0.12 & 0.18 & 0.19 \\
\hline Seychelles & Jan-94 & Dec-98 & yes & yes & SYM & 0.45 & 0.41 & 0.40 & 0.39 & 0.38 & 0.37 \\
\hline Seychelles & Jan-95 & Dec-99 & no & yes & SYM & 0.46 & 0.42 & 0.45 & 0.47 & 0.50 & 0.52 \\
\hline Seychelles & Jan-96 & Dec-00 & no & yes & SYM & 0.47 & 0.44 & 0.49 & 0.53 & 0.58 & 0.61 \\
\hline Seychelles & Jan-97 & Dec-01 & no & yes & SYM & 0.52 & 0.48 & 0.50 & 0.53 & 0.57 & 0.64 \\
\hline Seychelles & Jan-98 & Dec-02 & no & yes & SYM & 0.55 & 0.45 & 0.52 & 0.59 & 0.67 & 0.78 \\
\hline Seychelles & Jan-99 & Dec-03 & no & yes & SYM & -0.03 & 0.04 & 0.15 & 0.27 & 0.40 & 0.49 \\
\hline Seychelles & Jan-00 & Dec-04 & yes & yes & SYM & 0.03 & 0.15 & 0.36 & 0.48 & 0.52 & 0.52 \\
\hline Seychelles & Jan-01 & Dec-05 & yes & yes & SYM & 0.02 & 0.17 & 0.41 & 0.52 & 0.54 & 0.54 \\
\hline Swaziland & Jan-90 & Dec-94 & no & yes & SYM & 0.84 & 0.94 & 0.97 & 1.00 & 1.02 & 1.02 \\
\hline Swaziland & Jan-91 & Dec-95 & yes & yes & SYM & 0.84 & 0.95 & 0.98 & 0.99 & 0.99 & 0.99 \\
\hline
\end{tabular}


Table A-3: Multipliers of the pass-through model from national central bank to national retail rates

\begin{tabular}{|c|c|c|c|c|c|c|c|c|c|c|c|}
\hline \multirow[b]{2}{*}{ Country } & \multicolumn{2}{|c|}{ Sample period } & \multicolumn{2}{|c|}{ Cointegration? } & \multirow{2}{*}{$\begin{array}{c}\mathrm{PT} \\
\text { model }\end{array}$} & \multicolumn{6}{|c|}{ Multipliers } \\
\hline & Start & End & Engle/Granger & Kremers & & Impact & $1 \mathrm{mth}$ & 3 mths & 6 mths & 12 mths & Long-run \\
\hline Swaziland & Jan-92 & Dec-96 & yes & yes & SYM & 0.86 & 0.98 & 0.99 & 0.99 & 0.99 & 0.99 \\
\hline Swaziland & Jan-93 & Dec-97 & yes & yes & SYM & 0.86 & 0.98 & 0.99 & 0.99 & 0.99 & 0.99 \\
\hline Swaziland & Jan-94 & Dec-98 & yes & yes & SYM & 0.96 & 1.00 & 1.00 & 1.00 & 1.00 & 1.00 \\
\hline Swaziland & Jan-95 & Dec-99 & yes & yes & SYM & 0.97 & 1.01 & 1.00 & 1.00 & 1.00 & 1.00 \\
\hline Swaziland & Jan-96 & Dec-00 & no & yes & SYM & 1.00 & 1.00 & 1.00 & 1.00 & 1.00 & 1.00 \\
\hline Swaziland & Jan-97 & Dec-01 & no & no & STD & 1.00 & 1.00 & 1.00 & 1.00 & 1.00 & 1.00 \\
\hline Swaziland & Jan-98 & Dec-02 & no & no & STD & 1.00 & 1.00 & 1.00 & 1.00 & 1.00 & 1.00 \\
\hline Swaziland & Jan-99 & Dec-03 & no & no & STD & 0.98 & 0.96 & 0.96 & 0.96 & 0.96 & 0.96 \\
\hline Swaziland & Jan-00 & Dec-04 & no & no & STD & 0.97 & 0.93 & 0.93 & 0.93 & 0.93 & 0.93 \\
\hline Swaziland & Jan-01 & Dec-05 & no & no & STD & 0.97 & 0.93 & 0.93 & 0.93 & 0.93 & 0.93 \\
\hline Tanzania & Jan-92 & Dec-96 & yes & no & SYM & 0.09 & 0.12 & 0.16 & 0.21 & 0.25 & 0.28 \\
\hline Tanzania & Jan-93 & Dec-97 & no & yes & SYM & 0.08 & 0.10 & 0.13 & 0.17 & 0.23 & 0.33 \\
\hline Tanzania & Jan-94 & Dec-98 & no & no & SYM & 0.09 & 0.11 & 0.15 & 0.20 & 0.28 & 0.43 \\
\hline Tanzania & Jan-95 & Dec-99 & no & yes & SYM & 0.13 & 0.16 & 0.23 & 0.31 & 0.41 & 0.51 \\
\hline Tanzania & Jan-96 & Dec-00 & no & yes & SYM & 0.12 & 0.16 & 0.25 & 0.35 & 0.49 & 0.66 \\
\hline Tanzania & Jan-97 & Dec-01 & yes & yes & SYM & 0.10 & 0.10 & 0.11 & 0.11 & 0.12 & 0.13 \\
\hline Tanzania & Jan-98 & Dec-02 & no & yes & SYM & 0.13 & 0.14 & 0.17 & 0.20 & 0.24 & 0.36 \\
\hline Tanzania & Jan-99 & Dec-03 & no & no & STD & 0.08 & 0.09 & 0.09 & 0.09 & 0.09 & 0.09 \\
\hline Tanzania & Jan-00 & Dec-04 & no & no & STD & 0.13 & 0.13 & 0.13 & 0.13 & 0.13 & 0.13 \\
\hline Tanzania & Jan-01 & Dec-05 & no & no & STD & -0.05 & -0.05 & -0.05 & -0.05 & -0.05 & -0.05 \\
\hline Zambia & Jan-92 & Dec-96 & yes & yes & SYM & 0.63 & 0.91 & 1.06 & 1.06 & 1.06 & 1.06 \\
\hline Zambia & Jan-93 & Dec-97 & yes & yes & SYM & 0.48 & 0.75 & 0.95 & 0.98 & 0.97 & 0.97 \\
\hline Zambia & Jan-94 & Apr-98 & no & yes & SYM & 0.27 & 0.47 & 0.69 & 0.81 & 0.83 & 0.83 \\
\hline Zambia & Jan-95 & Dec-99 & n.a. & n.a. & n.a. & n.a. & n.a. & n.a. & n.a. & n.a. & n.a. \\
\hline Zambia & Jan-96 & Dec-00 & no & yes & SYM & 0.19 & 0.26 & 0.37 & 0.47 & 0.58 & 0.65 \\
\hline Zambia & Jan-97 & Dec-01 & no & no & SYM & 0.44 & 0.47 & 0.50 & 0.53 & 0.56 & 0.59 \\
\hline Zambia & Jan-98 & Dec-02 & yes & yes & SYM & 0.43 & 0.47 & 0.54 & 0.60 & 0.65 & 0.68 \\
\hline Zambia & Jan-99 & Dec-03 & yes & yes & SYM & 0.25 & 0.31 & 0.39 & 0.45 & 0.49 & 0.50 \\
\hline Zambia & Jan-00 & Dec-04 & yes & yes & SYM & 0.21 & 0.33 & 0.46 & 0.54 & 0.57 & 0.57 \\
\hline Zambia & Jan-01 & Dec-05 & no & yes & SYM & 0.22 & 0.31 & 0.45 & 0.56 & 0.65 & 0.68 \\
\hline Zimbabwe & Jan-90 & Dec-94 & no & yes & SYM & 0.01 & 0.15 & 0.38 & 0.61 & 0.86 & 1.02 \\
\hline Zimbabwe & Jan-91 & Dec-95 & no & yes & SYM & 0.03 & 0.23 & 0.49 & 0.75 & 1.01 & 1.18 \\
\hline Zimbabwe & Jan-92 & Dec-96 & yes & yes & SYM & -0.07 & 0.22 & 0.56 & 0.91 & 1.25 & 1.44 \\
\hline Zimbabwe & Jan-93 & Dec-97 & yes & yes & SYM & 0.30 & 0.53 & 0.64 & 0.66 & 0.66 & 0.66 \\
\hline Zimbabwe & Jan-94 & Dec-98 & yes & yes & SYM & 0.58 & 0.68 & 0.95 & 1.13 & 1.23 & 1.25 \\
\hline Zimbabwe & Jan-95 & Dec-99 & yes & yes & SYM & 0.55 & 0.46 & 0.57 & 0.64 & 0.72 & 0.76 \\
\hline Zimbabwe & Jan-96 & Dec-00 & no & no & STD & 0.38 & 0.38 & 0.38 & 0.38 & 0.38 & 0.38 \\
\hline Zimbabwe & Jan-97 & Dec-01 & no & no & STD & 0.31 & 0.42 & 0.47 & 0.48 & 0.48 & 0.48 \\
\hline Zimbabwe & Jan-98 & Dec-02 & no & no & STD & 0.13 & 0.18 & 0.20 & 0.20 & 0.20 & 0.20 \\
\hline Zimbabwe & Jan-99 & Dec-03 & yes & no & SYM & 0.94 & 0.72 & 0.74 & 0.71 & 0.64 & 1.18 \\
\hline Zimbabwe & Jan-00 & Dec-04 & yes & yes & SYM & 0.72 & 1.05 & 1.21 & 1.20 & 1.20 & 1.20 \\
\hline Zimbabwe & Jan-01 & Dec-05 & yes & yes & SYM & 0.61 & 0.74 & 0.84 & 0.90 & 0.93 & 0.93 \\
\hline
\end{tabular}

Note: The Zambian central bank rate is missing for all of 1999. Thus, in Panel A the pass-through (PT) is not estimated for the period 1995 to 1999. As the unit root tests generally indicate that the series are I(1), only the standard (STD) and symmetric (SYM) pass-through model are considered. SYM if chosen when at least one test indicates cointegration. To avoid switching too frequently from SYM to STD, i.e. for our overlapping sample periods, exceptions are made for single periods. These cases are highlighted in bold fond. For details on the methodology see Sander and Kleimeier (2004). 


\begin{tabular}{|c|c|c|c|c|c|c|c|c|c|c|c|}
\hline \multirow[b]{2}{*}{ Country } & \multicolumn{2}{|c|}{ Sample period } & \multicolumn{2}{|c|}{ Cointegration? } & \multirow{2}{*}{$\begin{array}{c}\text { PT } \\
\text { model }\end{array}$} & \multicolumn{6}{|c|}{ Multipliers } \\
\hline & Start & End & Engle/Granger & Kremers & & Impact & $1 \mathrm{mth}$ & 3 mths & 6 mths & 12 mths & Long-run \\
\hline \multicolumn{12}{|c|}{ Panel A: Pass-through from South African central bank rate to national deposit rate } \\
\hline South Africa & Jan-90 & Dec-94 & no & no & SYM & 0.66 & 0.88 & 1.04 & 1.14 & 1.26 & 1.35 \\
\hline South Africa & Jan-91 & Dec-95 & yes & no & SYM & 0.79 & 0.88 & 0.98 & 1.07 & 1.17 & 1.23 \\
\hline South Africa & Jan-92 & Dec-96 & yes & yes & SYM & 0.80 & 0.94 & 1.01 & 1.04 & 1.05 & 1.06 \\
\hline South Africa & Jan-93 & Dec-97 & yes & yes & SYM & 0.80 & 0.93 & 0.99 & 1.01 & 1.01 & 1.01 \\
\hline South Africa & Jan-94 & Dec-98 & yes & yes & SYM & 0.57 & 0.80 & 0.88 & 0.89 & 0.89 & 0.89 \\
\hline South Africa & Jan-95 & Dec-99 & yes & yes & SYM & 0.76 & 0.87 & 0.90 & 0.90 & 0.90 & 0.90 \\
\hline South Africa & Jan-96 & Dec-00 & yes & yes & SYM & 0.73 & 0.91 & 1.02 & 1.05 & 1.06 & 1.06 \\
\hline South Africa & Jan-97 & Dec-01 & yes & yes & SYM & 0.62 & 0.86 & 0.97 & 0.98 & 0.98 & 0.98 \\
\hline South Africa & Jan-98 & Dec-02 & yes & yes & SYM & 0.65 & 0.88 & 0.93 & 0.92 & 0.92 & 0.92 \\
\hline South Africa & Jan-99 & Dec-03 & yes & yes & SYM & 0.50 & 0.71 & 0.81 & 0.81 & 0.80 & 0.80 \\
\hline South Africa & Jan-00 & Dec-04 & no & yes & SYM & 0.52 & 0.73 & 0.82 & 0.81 & 0.81 & 0.81 \\
\hline South Africa & Jan-01 & Dec-05 & yes & yes & SYM & 0.56 & 0.81 & 0.91 & 0.87 & 0.86 & 0.86 \\
\hline Botswana & Jan-90 & Dec-94 & no & no & STD & -0.19 & -0.11 & -0.13 & -0.13 & -0.13 & -0.13 \\
\hline Botswana & Jan-91 & Dec-95 & no & no & STD & -0.16 & -0.11 & -0.12 & -0.12 & -0.12 & -0.12 \\
\hline Botswana & Jan-92 & Dec-96 & no & no & STD & -0.11 & -0.07 & -0.08 & -0.08 & -0.08 & -0.08 \\
\hline Botswana & Jan-93 & Dec-97 & yes & no & STD & -0.01 & 0.00 & 0.00 & 0.00 & 0.00 & 0.00 \\
\hline Botswana & Jan-94 & Dec-98 & yes & yes & STD & 0.01 & 0.01 & 0.01 & 0.01 & 0.01 & 0.01 \\
\hline Botswana & Jan-95 & Dec-99 & yes & yes & STD & -0.02 & -0.01 & -0.02 & -0.02 & -0.02 & -0.02 \\
\hline Botswana & Jan-96 & Dec-00 & no & no & STD & 0.00 & 0.00 & 0.00 & 0.00 & 0.00 & 0.00 \\
\hline Botswana & Jan-97 & Dec-01 & no & no & STD & -0.01 & -0.01 & -0.01 & -0.01 & -0.01 & -0.01 \\
\hline Botswana & Jan-98 & Dec-02 & no & no & STD & -0.01 & -0.01 & -0.01 & -0.01 & -0.01 & -0.01 \\
\hline Botswana & Jan-99 & Dec-03 & no & yes & SYM & 0.00 & 0.00 & -0.02 & -0.03 & -0.05 & -0.07 \\
\hline Botswana & Jan-00 & Dec-04 & no & yes & SYM & 0.02 & 0.03 & 0.04 & 0.05 & 0.06 & 0.06 \\
\hline Botswana & Jan-01 & Dec-05 & yes & yes & SYM & 0.04 & 0.07 & 0.11 & 0.14 & 0.15 & 0.15 \\
\hline Lesotho & Jan-90 & Dec-94 & yes & yes & STD & 0.31 & 0.30 & 0.30 & 0.30 & 0.30 & 0.30 \\
\hline Lesotho & Jan-91 & Dec-95 & no & no & STD & 0.59 & 0.58 & 0.58 & 0.58 & 0.58 & 0.58 \\
\hline Lesotho & Jan-92 & Dec-96 & no & no & STD & 0.43 & 0.41 & 0.41 & 0.41 & 0.41 & 0.41 \\
\hline Lesotho & Jan-93 & Dec-97 & no & no & STD & 0.41 & 0.41 & 0.41 & 0.41 & 0.41 & 0.41 \\
\hline Lesotho & Jan-94 & Dec-98 & no & yes & SYM & 0.32 & 0.31 & 0.31 & 0.30 & 0.28 & 0.27 \\
\hline Lesotho & Jan-95 & Dec-99 & no & no & SYM & 0.43 & 0.41 & 0.39 & 0.37 & 0.34 & 0.23 \\
\hline Lesotho & Jan-96 & Dec-00 & no & yes & SYM & 0.36 & 0.39 & 0.45 & 0.53 & 0.64 & 0.80 \\
\hline Lesotho & Jan-97 & Dec-01 & no & yes & SYM & 0.38 & 0.42 & 0.51 & 0.60 & 0.70 & 0.79 \\
\hline Lesotho & Jan-98 & Dec-02 & yes & yes & SYM & 0.32 & 0.42 & 0.54 & 0.63 & 0.67 & 0.68 \\
\hline Lesotho & Jan-99 & Dec-03 & yes & yes & SYM & 0.18 & 0.35 & 0.48 & 0.53 & 0.53 & 0.53 \\
\hline Lesotho & Jan-00 & Dec-04 & yes & yes & SYM & 0.21 & 0.19 & 0.21 & 0.22 & 0.22 & 0.22 \\
\hline Lesotho & Jan-01 & Dec-05 & yes & yes & SYM & 0.30 & 0.24 & 0.24 & 0.24 & 0.24 & 0.24 \\
\hline Namibia & Jan-91 & Dec-95 & yes & yes & SYM & 0.07 & 0.47 & 0.72 & 0.72 & 0.72 & 0.72 \\
\hline Namibia & Jan-92 & Dec-96 & yes & yes & SYM & 0.13 & 0.35 & 0.62 & 0.75 & 0.79 & 0.79 \\
\hline Namibia & Jan-93 & Dec-97 & yes & yes & SYM & 0.08 & 0.34 & 0.64 & 0.78 & 0.81 & 0.81 \\
\hline Namibia & Jan-94 & Dec-98 & yes & yes & SYM & 0.10 & 0.25 & 0.44 & 0.54 & 0.57 & 0.57 \\
\hline Namibia & Jan-95 & Dec-99 & yes & yes & SYM & 0.22 & 0.36 & 0.50 & 0.54 & 0.54 & 0.54 \\
\hline Namibia & Jan-96 & Dec-00 & no & yes & SYM & 0.25 & 0.42 & 0.60 & 0.70 & 0.74 & 0.75 \\
\hline Namibia & Jan-97 & Dec-01 & yes & yes & SYM & 0.23 & 0.39 & 0.59 & 0.71 & 0.77 & 0.78 \\
\hline Namibia & Jan-98 & Dec-02 & yes & yes & SYM & 0.18 & 0.37 & 0.60 & 0.71 & 0.74 & 0.75 \\
\hline Namibia & Jan-99 & Dec-03 & no & yes & SYM & 0.11 & 0.26 & 0.48 & 0.64 & 0.72 & 0.74 \\
\hline Namibia & Jan-00 & Dec-04 & no & yes & SYM & -0.01 & 0.06 & 0.18 & 0.29 & 0.36 & 0.38 \\
\hline Namibia & Jan-01 & Dec-05 & no & yes & SYM & 0.00 & 0.08 & 0.21 & 0.31 & 0.37 & 0.38 \\
\hline Seychelles & Jan-90 & Dec-94 & no & no & STD & -0.06 & -0.06 & -0.06 & -0.06 & -0.06 & -0.06 \\
\hline Seychelles & Jan-91 & Dec-95 & no & no & STD & -0.05 & -0.05 & -0.05 & -0.05 & -0.05 & -0.05 \\
\hline Seychelles & Jan-92 & Dec-96 & yes & yes & SYM & 0.01 & 0.05 & 0.13 & 0.17 & 0.19 & 0.19 \\
\hline Seychelles & Jan-93 & Dec-97 & no & yes & SYM & 0.04 & 0.06 & 0.08 & 0.10 & 0.11 & 0.11 \\
\hline Seychelles & Jan-94 & Dec-98 & no & yes & SYM & -0.02 & -0.03 & -0.06 & -0.09 & -0.13 & -0.18 \\
\hline Seychelles & Jan-95 & Dec-99 & no & yes & SYM & 0.00 & 0.00 & 0.00 & 0.00 & 0.00 & 0.02 \\
\hline Seychelles & Jan-96 & Dec-00 & no & yes & SYM & 0.00 & 0.00 & -0.01 & -0.01 & -0.03 & 0.37 \\
\hline
\end{tabular}


Table A-4: The pass-through from South African central bank rates to retail rates of Group 1 countries

\begin{tabular}{|c|c|c|c|c|c|c|c|c|c|c|c|}
\hline \multirow[b]{2}{*}{ Country } & \multicolumn{2}{|c|}{ Sample period } & \multicolumn{2}{|c|}{ Cointegration? } & \multirow{2}{*}{$\begin{array}{c}\mathrm{PT} \\
\text { model }\end{array}$} & \multicolumn{6}{|c|}{ Multipliers } \\
\hline & Start & End & Engle/Granger & Kremers & & Impact & $1 \mathrm{mth}$ & 3 mths & 6 mths & 12 mths & Long-run \\
\hline Seychelles & Jan-97 & Dec-01 & no & yes & SYM & -0.05 & -0.05 & -0.05 & -0.05 & -0.05 & 0.35 \\
\hline Seychelles & Jan-98 & Dec-02 & yes & yes & SYM & 0.07 & 0.10 & 0.14 & 0.18 & 0.22 & 0.23 \\
\hline Seychelles & Jan-99 & Dec-03 & yes & yes & SYM & 0.09 & 0.12 & 0.13 & 0.13 & 0.14 & 0.14 \\
\hline Seychelles & Jan-00 & Dec-04 & yes & yes & SYM & 0.09 & 0.12 & 0.16 & 0.19 & 0.22 & 0.22 \\
\hline Seychelles & Jan-01 & Dec-05 & yes & yes & SYM & 0.08 & 0.10 & 0.13 & 0.16 & 0.19 & 0.20 \\
\hline Swaziland & Jan-90 & Dec-94 & no & no & SYM & 0.26 & 0.32 & 0.34 & 0.35 & 0.36 & 0.38 \\
\hline Swaziland & Jan-91 & Dec-95 & yes & yes & SYM & 0.25 & 0.36 & 0.50 & 0.60 & 0.67 & 0.68 \\
\hline Swaziland & Jan-92 & Dec-96 & yes & yes & SYM & 0.41 & 0.69 & 0.89 & 0.94 & 0.94 & 0.94 \\
\hline Swaziland & Jan-93 & Dec-97 & yes & yes & SYM & 0.43 & 0.83 & 0.99 & 1.01 & 1.01 & 1.01 \\
\hline Swaziland & Jan-94 & Dec-98 & no & yes & SYM & 0.25 & 0.33 & 0.47 & 0.59 & 0.66 & 0.68 \\
\hline Swaziland & Jan-95 & Dec-99 & yes & yes & SYM & 0.25 & 0.39 & 0.54 & 0.61 & 0.63 & 0.63 \\
\hline Swaziland & Jan-96 & Dec-00 & no & yes & SYM & 0.32 & 0.45 & 0.59 & 0.69 & 0.76 & 0.77 \\
\hline Swaziland & Jan-97 & Dec-01 & no & yes & SYM & 0.30 & 0.43 & 0.59 & 0.71 & 0.77 & 0.78 \\
\hline Swaziland & Jan-98 & Dec-02 & no & yes & SYM & 0.35 & 0.47 & 0.59 & 0.67 & 0.71 & 0.72 \\
\hline Swaziland & Jan-99 & Dec-03 & no & yes & SYM & 0.58 & 0.74 & 0.81 & 0.85 & 0.88 & 0.89 \\
\hline Swaziland & Jan-00 & Dec-04 & no & no & SYM & 0.69 & 0.69 & 0.69 & 0.69 & 0.69 & 0.69 \\
\hline Swaziland & Jan-01 & Dec-05 & yes & yes & SYM & 0.70 & 0.70 & 0.71 & 0.71 & 0.73 & 0.74 \\
\hline \multicolumn{12}{|c|}{ Panel B: Pass-through from South African central bank rate to national loan rate } \\
\hline South Africa & Jan-90 & Dec-94 & yes & yes & SYM & 0.32 & 0.91 & 0.97 & 0.96 & 0.96 & 0.96 \\
\hline South Africa & Jan-91 & Dec-95 & yes & yes & SYM & 0.44 & 1.01 & 0.99 & 0.98 & 0.98 & 0.98 \\
\hline South Africa & Jan-92 & Dec-96 & yes & yes & SYM & 0.62 & 1.14 & 1.07 & 1.05 & 1.05 & 1.05 \\
\hline South Africa & Jan-93 & Dec-97 & yes & yes & SYM & 0.81 & 1.10 & 1.04 & 1.01 & 1.02 & 1.02 \\
\hline South Africa & Jan-94 & Dec-98 & yes & yes & SYM & 0.45 & 0.96 & 1.01 & 0.98 & 0.98 & 0.98 \\
\hline South Africa & Jan-95 & Dec-99 & yes & yes & SYM & 0.42 & 0.98 & 0.98 & 0.95 & 0.95 & 0.95 \\
\hline South Africa & Jan-96 & Dec-00 & yes & yes & SYM & 0.49 & 0.96 & 1.06 & 1.03 & 1.04 & 1.04 \\
\hline South Africa & Jan-97 & Dec-01 & yes & yes & SYM & 0.47 & 0.94 & 1.04 & 1.03 & 1.03 & 1.03 \\
\hline South Africa & Jan-98 & Dec-02 & yes & yes & SYM & 0.59 & 0.91 & 1.02 & 1.01 & 1.01 & 1.01 \\
\hline South Africa & Jan-99 & Dec-03 & yes & yes & SYM & 0.85 & 0.86 & 0.93 & 0.98 & 1.01 & 1.02 \\
\hline South Africa & Jan-00 & Dec-04 & no & yes & SYM & 0.87 & 0.91 & 0.92 & 0.92 & 0.92 & 0.93 \\
\hline South Africa & Jan-01 & Dec-05 & no & no & SYM & 0.89 & 0.93 & 0.94 & 0.95 & 0.95 & 0.96 \\
\hline Angola & Jan-95 & Dec-99 & yes & yes & SYM & -8.09 & -9.90 & -12.78 & -15.37 & -17.49 & -18.34 \\
\hline Angola & Jan-96 & Dec-00 & yes & yes & SYM & -7.43 & -7.43 & -8.11 & -8.57 & -8.84 & -8.89 \\
\hline Angola & Jan-97 & Dec-01 & yes & yes & SYM & -3.25 & -4.79 & -6.35 & -7.28 & -7.69 & -7.74 \\
\hline Angola & Jan-98 & Dec-02 & yes & yes & SYM & -2.81 & -4.31 & -5.74 & -6.45 & -6.69 & -6.71 \\
\hline Angola & Jan-99 & Dec-03 & yes & yes & SYM & -3.02 & -4.22 & -5.15 & -5.53 & -5.63 & -5.63 \\
\hline Angola & Jan-00 & Dec-04 & yes & yes & SYM & -1.76 & 0.15 & 1.97 & 2.41 & 2.45 & 2.45 \\
\hline Angola & Jan-01 & Dec-05 & yes & yes & SYM & -2.59 & -1.04 & 0.91 & 2.50 & 3.62 & 3.95 \\
\hline Lesotho & Jan-90 & Dec-94 & yes & yes & SYM & 0.45 & 1.02 & 1.02 & 1.02 & 1.02 & 1.02 \\
\hline Lesotho & Jan-91 & Dec-95 & yes & yes & SYM & 0.45 & 0.89 & 1.03 & 1.05 & 1.05 & 1.05 \\
\hline Lesotho & Jan-92 & Dec-96 & yes & yes & SYM & 0.14 & 0.79 & 0.89 & 0.90 & 0.90 & 0.90 \\
\hline Lesotho & Jan-93 & Dec-97 & yes & yes & SYM & 0.34 & 0.77 & 0.74 & 0.74 & 0.74 & 0.74 \\
\hline Lesotho & Jan-94 & Dec-98 & yes & yes & SYM & 0.22 & 0.81 & 0.93 & 0.90 & 0.90 & 0.90 \\
\hline Lesotho & Jan-95 & Dec-99 & yes & yes & SYM & 0.16 & 0.36 & 0.58 & 0.65 & 0.66 & 0.66 \\
\hline Lesotho & Jan-96 & Dec-00 & yes & yes & SYM & 0.13 & 0.28 & 0.42 & 0.47 & 0.48 & 0.48 \\
\hline Lesotho & Jan-97 & Dec-01 & yes & yes & SYM & 0.22 & 0.34 & 0.43 & 0.46 & 0.47 & 0.47 \\
\hline Lesotho & Jan-98 & Dec-02 & yes & yes & SYM & 0.08 & 0.35 & 0.55 & 0.53 & 0.53 & 0.53 \\
\hline Lesotho & Jan-99 & Dec-03 & yes & yes & SYM & 0.51 & 0.64 & 0.69 & 0.70 & 0.70 & 0.70 \\
\hline Lesotho & Jan-00 & Dec-04 & yes & yes & SYM & 0.41 & 0.56 & 0.70 & 0.81 & 0.90 & 0.95 \\
\hline Lesotho & Jan-01 & Dec-05 & yes & yes & SYM & 0.42 & 0.57 & 0.71 & 0.82 & 0.93 & 0.98 \\
\hline Malawi & Jan-90 & Dec-94 & yes & yes & STD & 0.00 & 0.00 & 0.00 & 0.00 & 0.00 & 0.00 \\
\hline Malawi & Jan-91 & Dec-95 & no & no & STD & 0.00 & 0.00 & 0.00 & 0.00 & 0.00 & 0.00 \\
\hline Malawi & Jan-92 & Dec-96 & no & no & STD & 0.10 & 0.11 & 0.11 & 0.11 & 0.11 & 0.11 \\
\hline Malawi & Jan-93 & Dec-97 & no & no & STD & 0.07 & 0.07 & 0.07 & 0.07 & 0.07 & 0.07 \\
\hline Malawi & Jan-94 & Dec-98 & no & no & STD & -0.42 & -0.46 & -0.47 & -0.47 & -0.47 & -0.47 \\
\hline Malawi & Jan-95 & Dec-99 & no & no & STD & -0.42 & -0.45 & -0.45 & -0.45 & -0.45 & -0.45 \\
\hline
\end{tabular}


Table A-4: The pass-through from South African central bank rates to retail rates of Group 1 countries

\begin{tabular}{|c|c|c|c|c|c|c|c|c|c|c|c|}
\hline \multirow[b]{2}{*}{ Country } & \multicolumn{2}{|c|}{ Sample period } & \multicolumn{2}{|c|}{ Cointegration? } & \multirow{2}{*}{$\begin{array}{c}\mathrm{PT} \\
\text { model }\end{array}$} & \multicolumn{6}{|c|}{ Multipliers } \\
\hline & Start & End & Engle/Granger & Kremers & & Impact & $1 \mathrm{mth}$ & $3 \mathrm{mths}$ & $6 \mathrm{mths}$ & 12 mths & Long-run \\
\hline Malawi & Jan-96 & Dec-00 & no & no & STD & -0.29 & -0.35 & -0.37 & -0.37 & -0.37 & -0.37 \\
\hline Malawi & Jan-97 & Dec-01 & no & no & STD & -0.51 & -0.52 & -0.52 & -0.52 & -0.52 & -0.52 \\
\hline Malawi & Jan-98 & Dec-02 & yes & yes & SYM & -1.10 & -1.19 & -1.25 & -1.30 & -1.35 & -1.37 \\
\hline Malawi & Jan-99 & Dec-03 & no & yes & SYM & -0.20 & -0.16 & -0.06 & 0.03 & 0.13 & 0.18 \\
\hline Malawi & Jan-00 & Dec-04 & no & yes & SYM & -0.30 & -0.02 & 0.52 & 1.11 & 1.80 & 2.41 \\
\hline Malawi & Jan-01 & Dec-05 & no & yes & SYM & -0.29 & 0.09 & 0.73 & 1.44 & 2.26 & 2.99 \\
\hline Namibia & Jan-91 & Dec-95 & yes & yes & SYM & 0.01 & 0.43 & 0.77 & 1.01 & 1.13 & 1.15 \\
\hline Namibia & Jan-92 & Dec-96 & yes & yes & SYM & -0.84 & 0.38 & 0.56 & 0.62 & 0.63 & 0.63 \\
\hline Namibia & Jan-93 & Dec-97 & yes & yes & SYM & -0.64 & 0.40 & 0.61 & 0.59 & 0.59 & 0.59 \\
\hline Namibia & Jan-94 & Dec-98 & yes & yes & SYM & -0.18 & 0.48 & 0.55 & 0.56 & 0.56 & 0.56 \\
\hline Namibia & Jan-95 & Dec-99 & yes & yes & SYM & -0.03 & 0.49 & 0.57 & 0.58 & 0.58 & 0.58 \\
\hline Namibia & Jan-96 & Dec-00 & yes & yes & SYM & 0.05 & 0.50 & 0.67 & 0.73 & 0.73 & 0.73 \\
\hline Namibia & Jan-97 & Dec-01 & yes & yes & SYM & 0.19 & 0.48 & 0.68 & 0.77 & 0.79 & 0.79 \\
\hline Namibia & Jan-98 & Dec-02 & yes & yes & SYM & 0.09 & 0.40 & 0.62 & 0.75 & 0.81 & 0.81 \\
\hline Namibia & Jan-99 & Dec-03 & yes & yes & SYM & -0.02 & 0.36 & 0.62 & 0.77 & 0.84 & 0.84 \\
\hline nibia & Jan-00 & Dec-04 & yes & yes & SYM & -0.19 & 0.28 & 0.53 & 0.65 & 0.69 & 0.69 \\
\hline Namibia & Jan-01 & Dec-05 & yes & yes & SYM & -0.24 & 0.35 & 0.58 & 0.69 & 0.72 & 0.72 \\
\hline Seychelles & Jan-90 & Dec-94 & no & yes & SYM & 0.00 & 0.00 & -0.01 & -0.01 & -0.02 & -0.02 \\
\hline Seychelles & Jan-91 & Dec-95 & no & yes & SYM & 0.02 & 0.01 & -0.01 & -0.02 & -0.03 & -0.03 \\
\hline Seychelles & Jan-92 & Dec-96 & no & no & SYM & 0.09 & 0.07 & 0.07 & 0.07 & 0.07 & 0.06 \\
\hline Seychelles & n-93 & Dec-97 & no & yes & SYM & 0.02 & 0.01 & -0.02 & -0.04 & -0.06 & -0.07 \\
\hline Seychelles & Jan-94 & Dec-98 & yes & yes & SYM & 0.02 & -0.03 & -0.10 & -0.16 & -0.20 & -0.20 \\
\hline Seychelles & Jan-95 & Dec-99 & no & yes & SYM & 0.00 & 0.00 & 0.00 & 0.00 & 0.00 & 0.05 \\
\hline Seychelles & Jan-96 & Dec-00 & no & yes & SYM & -0.01 & -0.02 & -0.03 & -0.05 & -0.09 & 0.37 \\
\hline Seychelles & Jan-97 & Dec-01 & no & yes & SYM & -0.04 & -0.05 & -0.07 & -0.09 & -0.16 & 0.37 \\
\hline Seychelles & Jan-98 & Dec-02 & yes & yes & SYM & 0.04 & 0.06 & 0.09 & 0.13 & 0.19 & 0.31 \\
\hline elles & Jan-99 & Dec-03 & yes & yes & SYM & 0.01 & 0.05 & 0.11 & 0.15 & 0.17 & 0.17 \\
\hline Seychelles & Jan-00 & Dec-04 & no & yes & SYM & 0.02 & 0.05 & 0.10 & 0.14 & 0.18 & 0.19 \\
\hline Seychelles & Jan-01 & Dec-05 & yes & yes & SYM & 0.01 & 0.05 & 0.12 & 0.17 & 0.19 & 0.20 \\
\hline Swaziland & Jan-90 & Dec-94 & no & yes & STD & 0.16 & 0.18 & 0.18 & 0.18 & 0.18 & 0.18 \\
\hline Swaziland & Jan-91 & Dec-95 & no & no & STD & 0.12 & 0.14 & 0.14 & 0.14 & 0.14 & 0.14 \\
\hline Swaziland & Jan-92 & Dec-96 & no & no & STD & 0.21 & 0.22 & 0.23 & 0.23 & 0.23 & 0.23 \\
\hline Swaziland & Jan-93 & Dec-97 & yes & yes & SYM & 0.44 & 0.85 & 1.18 & 1.23 & 1.23 & 1.23 \\
\hline Swaziland & Jan-94 & Dec-98 & no & yes & SYM & 0.26 & 0.35 & 0.50 & 0.63 & 0.73 & 0.77 \\
\hline Swaziland & Jan-95 & Dec-99 & yes & yes & SYM & 0.25 & 0.39 & 0.54 & 0.61 & 0.64 & 0.64 \\
\hline Swaziland & Jan-96 & Dec-00 & no & yes & SYM & 0.33 & 0.44 & 0.57 & 0.68 & 0.76 & 0.78 \\
\hline Swaziland & Jan-97 & Dec-01 & no & yes & SYM & 0.34 & 0.45 & 0.60 & 0.72 & 0.80 & 0.83 \\
\hline Swaziland & Jan-98 & Dec-02 & yes & yes & SYM & 0.39 & 0.49 & 0.61 & 0.70 & 0.75 & 0.76 \\
\hline Swaziland & Jan-99 & Dec-03 & yes & yes & SYM & 0.75 & 0.84 & 0.91 & 0.96 & 0.99 & 0.99 \\
\hline Swaziland & Jan-00 & Dec-04 & no & no & SYM & 0.86 & 0.84 & 0.84 & 0.84 & 0.84 & 0.84 \\
\hline Swaziland & Jan-01 & Dec-05 & no & yes & SYM & 0.87 & 0.85 & 0.86 & 0.86 & 0.86 & 0.86 \\
\hline
\end{tabular}

See notes to Table A-3. 
Table B-1: Principal components analysis of bank rates convergence for the SADC countries for the rolling periods

\begin{tabular}{|c|c|c|c|c|c|c|c|c|c|c|c|c|c|c|c|c|c|c|c|c|c|c|}
\hline \multirow{3}{*}{$\begin{array}{l}\text { Countries } \\
\text { Panel A: SADC }\end{array}$} & \multirow{3}{*}{$\begin{array}{r}\text { Com- } \\
\text { ponent }\end{array}$} & \multicolumn{3}{|c|}{ 1990-1994 } & \multicolumn{3}{|c|}{ 1991-1995 } & \multicolumn{3}{|c|}{$1992-1996$} & \multicolumn{3}{|c|}{$\begin{array}{l}1993-1997 \\
\end{array}$} & \multicolumn{3}{|c|}{ 1994-1998 } & \multicolumn{3}{|c|}{ 1995-1999 } & \multicolumn{3}{|c|}{ 1996-2000 } \\
\hline & & $\begin{array}{l}\text { Eigen- } \\
\text { value }\end{array}$ & Cum $R^{2}$ & Vector 1 & $\begin{array}{c}\text { Eigen- } \\
\text { value }\end{array}$ & Cum $R^{2}$ & Vector 1 & $\begin{array}{l}\text { Eigen- } \\
\text { value }\end{array}$ & Cum $R^{2}$ & Vector 1 & $\begin{array}{l}\text { Eigen- } \\
\text { value }\end{array}$ & Cum $R^{2} V$ & Vector 1 & $\begin{array}{l}\text { Eigen- } \\
\text { value }\end{array}$ & $\mathrm{Cum}^{2}$ & Vector 1 & $\begin{array}{r}\text { Eigen- } \\
\text { value }\end{array}$ & $\mathrm{Cum}^{2}$ & Vector 1 & $\begin{array}{l}\text { Eigen- } \\
\text { value }\end{array}$ & Cum $R^{2}$ & Vector 1 \\
\hline & & & & & & & & & & & & & & & & & & & & & & \\
\hline Angola & 1 & 5.717 & 0.635 & & 4.921 & 0.492 & & 5.546 & 0.462 & & 6.137 & 0.472 & & 6.381 & 0.532 & & 5.818 & 0.448 & -0.304 & 5.849 & 0.450 & -0.277 \\
\hline Botswana & 2 & 1.699 & 0.824 & -0.289 & 3.219 & 0.814 & -0.276 & 3.049 & 0.716 & -0.275 & 3.564 & 0.746 & -0.117 & 2.729 & 0.759 & -0.310 & 3.589 & 0.724 & -0.302 & 3.847 & 0.746 & -0.329 \\
\hline DR Congo & 3 & 0.979 & 0.934 & -0.356 & 1.094 & 0.923 & -0.397 & 1.112 & 0.809 & 0.289 & 1.295 & 0.846 & 0.863 & 1.393 & 0.875 & -0.238 & 1.534 & 0.842 & -0.258 & 1.306 & 0.846 & -0.034 \\
\hline Lesotho & 4 & 0.201 & 0.955 & 0.360 & 0.349 & 0.958 & 0.363 & 0.923 & 0.886 & 0.178 & 0.758 & 0.904 & -0.146 & 0.495 & 0.917 & 0.333 & 0.839 & 0.906 & 0.259 & 0.752 & 0.904 & -0.163 \\
\hline Madagascar & 5 & 0.154 & 0.973 & & 0.144 & 0.973 & & 0.540 & 0.931 & 0.327 & 0.489 & 0.942 & -0.125 & 0.435 & 0.953 & -0.265 & 0.567 & 0.950 & -0.380 & 0.530 & 0.945 & 0.042 \\
\hline Malawi & 6 & 0.107 & 0.984 & -0.393 & 0.116 & 0.984 & -0.300 & 0.350 & 0.960 & 0.286 & 0.310 & 0.966 & -0.160 & 0.205 & 0.970 & -0.126 & 0.237 & 0.968 & -0.259 & 0.248 & 0.964 & -0.293 \\
\hline Mauritius & 7 & 0.079 & 0.993 & 0.182 & 0.069 & 0.991 & -0.382 & 0.204 & 0.977 & 0.295 & 0.161 & 0.978 & 0.201 & 0.162 & 0.983 & -0.363 & 0.146 & 0.979 & & 0.219 & 0.981 & \\
\hline Mozambique & 8 & 0.053 & 0.999 & & 0.051 & 0.996 & & 0.128 & 0.988 & & 0.110 & 0.986 & & 0.075 & 0.990 & & 0.089 & 0.986 & -0.371 & 0.093 & 0.988 & 0.175 \\
\hline Namibia & 9 & 0.013 & 1 & & 0.023 & 0.999 & & 0.090 & 0.995 & 0.296 & 0.076 & 0.992 & -0.112 & 0.057 & 0.994 & 0.278 & 0.072 & 0.992 & 0.111 & 0.065 & 0.993 & 0.381 \\
\hline Seychelles & 10 & & & 0.406 & 0.013 & 1.000 & -0.005 & 0.027 & 0.997 & 0.335 & 0.052 & 0.996 & -0.071 & 0.036 & 0.997 & 0.351 & 0.057 & 0.996 & 0.286 & 0.042 & 0.996 & 0.342 \\
\hline South Africa & 11 & & & 0.220 & & & 0.341 & 0.018 & 0.999 & -0.290 & 0.026 & 0.998 & -0.061 & 0.020 & 0.999 & -0.350 & 0.029 & 0.998 & -0.238 & 0.028 & 0.998 & 0.324 \\
\hline Swaziland & 12 & & & 0.302 & & & 0.371 & 0.013 & 1.000 & 0.393 & 0.015 & 0.999 & -0.081 & 0.011 & 1.000 & 0.312 & 0.016 & 0.999 & 0.269 & 0.016 & 1.000 & 0.370 \\
\hline Tanzania & 13 & & & & & & & & & & 0.008 & 1.000 & -0.259 & & & -0.287 & 0.007 & 1.000 & -0.331 & 0.006 & 1.000 & 0.100 \\
\hline Zambia & 14 & & & & & & 0.036 & & & -0.305 & & & 0.200 & & & & & & & & & \\
\hline Zimbabwe & 15 & & & -0.362 & & & -0.378 & & & -0.048 & & & -0.058 & & & 0.127 & & & 0.029 & & & -0.395 \\
\hline No of factors & & & 2 & & & 3 & & & 3 & & & 3 & & & 3 & & & 3 & & & 3 & \\
\hline Countries excluded & & ANG, $\mathrm{M}$ & $\begin{array}{l}\text { IAD, MC } \\
\text { TA, ZA }\end{array}$ & $\mathrm{OZ}, \mathrm{NA}$, & ANG, M & $\begin{array}{c}\text { IAD, MO } \\
\text { TA } \\
\end{array}$ & $\mathrm{Z}, \mathrm{NA}$ & ANC & $\mathrm{G}, \mathrm{MOZ}$ & TA & & $\mathrm{NG}, \mathrm{MOZ}$ & & ANC & $\mathrm{G}, \mathrm{MOZ}$ & ZA & & MAU, ZA & & & MAU, ZA & \\
\hline Panel B: SACU & & & & & & & & & & & & & & & & & & & & & & \\
\hline$\overline{\text { Botswana }}$ & 1 & 2.826 & 0.706 & -0.384 & 2.821 & 0.705 & -0.445 & 3.603 & 0.721 & -0.294 & 4.156 & 0.831 & -0.351 & 3.936 & 0.787 & -0.299 & 3.048 & 0.610 & -0.348 & 3.402 & 0.680 & -0.427 \\
\hline Lesotho & 2 & 0.955 & 0.945 & 0.534 & 0.698 & 0.880 & 0.529 & 0.874 & 0.896 & 0.438 & 0.632 & 0.958 & 0.446 & 0.773 & 0.942 & 0.474 & 0.973 & 0.804 & 0.229 & 1.094 & 0.899 & -0.076 \\
\hline Namibia & 3 & 0.184 & 0.991 & & 0.398 & 0.979 & & 0.398 & 0.975 & 0.505 & 0.156 & 0.989 & 0.455 & 0.179 & 0.978 & 0.469 & 0.751 & 0.955 & 0.484 & 0.397 & 0.979 & 0.527 \\
\hline South Africa & 4 & 0.035 & 1.000 & 0.574 & 0.083 & 1.000 & 0.577 & 0.097 & 0.994 & 0.517 & 0.037 & 0.996 & 0.484 & 0.072 & 0.992 & 0.494 & 0.184 & 0.991 & 0.556 & 0.077 & 0.994 & 0.514 \\
\hline Swaziland & 5 & & & 0.488 & & & 0.435 & 0.028 & 1.000 & 0.447 & 0.018 & 1.000 & 0.486 & 0.040 & 1.000 & 0.471 & 0.043 & 1.000 & 0.532 & 0.030 & 1.000 & 0.520 \\
\hline No of factors & & & 1 & & & 1 & & & 1 & & & 1 & & & 1 & & & 1 & & & 2 & \\
\hline$\frac{\text { Countries excluded }}{\text { Panel C: CMA }}$ & & & NA & & & NA & & & & & & & & & & & & & & & & \\
\hline Lesotho & 1 & 2.522 & 0.841 & -0.590 & 2.376 & 0.792 & -0.588 & 3.358 & 0.840 & -0.475 & 3.713 & 0.928 & -0.486 & 3.647 & 0.912 & -0.499 & 2.768 & 0.692 & -0.259 & 2.869 & 0.717 & -0.037 \\
\hline Namibia & 2 & 0.294 & 0.939 & -0.567 & 0.507 & 0.961 & -0.619 & 0.509 & 0.967 & -0.528 & 0.163 & 0.969 & -0.495 & 0.207 & 0.964 & -0.504 & 0.963 & 0.933 & -0.528 & 1.023 & 0.973 & 0.582 \\
\hline South Africa & 3 & 0.184 & 1.000 & & 0.117 & 1.000 & & 0.104 & 0.993 & -0.537 & 0.103 & 0.995 & -0.511 & 0.090 & 0.986 & -0.513 & 0.208 & 0.985 & -0.587 & 0.077 & 0.992 & 0.575 \\
\hline Swaziland & 4 & & & -0.575 & & & -0.521 & 0.028 & 1.000 & -0.455 & 0.021 & 1.000 & -0.507 & 0.056 & 1.000 & -0.484 & 0.060 & 1.000 & -0.556 & 0.030 & 1.000 & 0.574 \\
\hline $\begin{array}{l}\text { No of factors } \\
\text { Countries excl }\end{array}$ & & & $\begin{array}{c}1 \\
\mathrm{NA}\end{array}$ & & & $\begin{array}{c}1 \\
\mathrm{NA}\end{array}$ & & & 1 & & & 1 & & & 1 & & & 1 & & & 2 & \\
\hline $\begin{array}{l}\text { Countries excluded } \\
\text { Panel D: OTHER }\end{array}$ & & & & & & & & & & & & & & & & & & & & & & \\
\hline Angola & 1 & 3.185 & 0.637 & & 3.933 & 0.656 & & 3.878 & 0.554 & & 3.686 & 0.461 & & 3.958 & 0.565 & -0.361 & 4.377 & 0.547 & -0.327 & 3.652 & 0.456 & -0.062 \\
\hline DR Congo & 2 & 1.402 & 0.917 & -0.516 & 1.361 & 0.882 & -0.454 & 1.348 & 0.747 & -0.428 & 2.269 & 0.744 & -0.318 & 1.617 & 0.796 & -0.469 & 2.094 & 0.809 & -0.301 & 2.795 & 0.806 & -0.374 \\
\hline Madagascar & 3 & 0.196 & 0.956 & & 0.412 & 0.951 & & 0.825 & 0.864 & -0.455 & 0.888 & 0.855 & -0.471 & 0.741 & 0.902 & -0.342 & 0.811 & 0.910 & -0.468 & 0.690 & 0.892 & -0.483 \\
\hline Malawi & 4 & 0.117 & 0.980 & -0.535 & 0.199 & 0.984 & -0.457 & 0.484 & 0.934 & -0.445 & 0.548 & 0.924 & -0.460 & 0.341 & 0.951 & -0.485 & 0.359 & 0.955 & -0.258 & 0.469 & 0.951 & -0.032 \\
\hline Mauritius & 5 & 0.101 & 1.000 & 0.144 & 0.071 & 0.996 & -0.480 & 0.277 & 0.973 & -0.380 & 0.310 & 0.963 & -0.416 & 0.251 & 0.987 & & 0.220 & 0.983 & & 0.253 & 0.982 & \\
\hline Mozambique & 6 & & & & 0.023 & 1.000 & & 0.167 & 0.997 & & 0.160 & 0.983 & & 0.057 & 0.995 & & 0.087 & 0.993 & -0.467 & 0.080 & 0.992 & -0.514 \\
\hline Seychelles & 7 & & & 0.461 & & & -0.188 & 0.021 & 1.000 & 0.362 & 0.110 & 0.996 & 0.033 & 0.034 & 1.000 & -0.379 & 0.034 & 0.998 & -0.352 & 0.043 & 0.998 & -0.404 \\
\hline Tanzania & 8 & & & & & & & & & & 0.029 & 1.000 & -0.399 & & & -0.372 & 0.018 & 1.000 & -0.384 & 0.018 & 1.000 & -0.355 \\
\hline Zambia & 9 & & & & & & 0.482 & & & 0.337 & & & 0.307 & & & & & & & & & \\
\hline Zimbabwe & 10 & & & -0.462 & & & -0.295 & & & -0.153 & & & -0.194 & & & 0.124 & & & 0.167 & & & 0.264 \\
\hline No of factors & & & 2 & & & 2 & & & 2 & & & 2 & & & 2 & & & 2 & & & 2 & \\
\hline Countries excluded & & ANG, M & $\begin{array}{l}\text { IAD, MC } \\
\text { TA, ZA }\end{array}$ & DZ, NA, & ANG, M & $\mathrm{AAD}, \mathrm{MC}$ & $\mathrm{ZZ}, \mathrm{TA}$ & ANC & $\mathrm{G}, \mathrm{MOZ}$ & TA & & $\mathrm{NG}, \mathrm{MOZ}$ & & MAI & $\mathrm{U}, \mathrm{ZA}, \mathrm{M}$ & & & MAU, ZA & & & MAU, ZA & \\
\hline
\end{tabular}


Table B-1 continued: Principal components analysis of bank rates convergence for the SADC countries for the rolling periods

\begin{tabular}{|c|c|c|c|c|c|c|c|c|c|c|c|c|c|c|c|c|}
\hline \multirow[b]{2}{*}{ Countries } & \multirow[b]{2}{*}{$\begin{array}{l}\text { Com- } \\
\text { ponent }\end{array}$} & \multicolumn{3}{|c|}{$1997-2001$} & \multicolumn{3}{|c|}{$1998-2002$} & \multicolumn{3}{|c|}{$1999-2003$} & \multicolumn{3}{|c|}{$2000-2004$} & \multicolumn{3}{|c|}{$2001-2005$} \\
\hline & & $\begin{array}{l}\text { Eigen- } \\
\text { value }\end{array}$ & $\mathrm{Cum} \mathrm{R}^{2}$ & Vector 1 & $\begin{array}{l}\text { Eigen- } \\
\text { value }\end{array}$ & $\mathrm{Cum} \mathrm{R}^{2}$ & Vector 1 & $\begin{array}{l}\text { Eigen- } \\
\text { value }\end{array}$ & Cum $R^{2}$ & Vector 1 & $\begin{array}{l}\text { Eigen- } \\
\text { value }\end{array}$ & $\operatorname{Cum~R}^{2}$ & Vector 1 & $\begin{array}{l}\text { Eigen- } \\
\text { value }\end{array}$ & Cum $R^{2} Y$ & Vector 1 \\
\hline \multicolumn{17}{|l|}{ Panel A: SADC } \\
\hline Angola & 1 & 7.512 & 0.578 & -0.348 & 7.277 & 0.560 & -0.346 & 4.794 & 0.399 & -0.395 & 6.307 & 0.526 & -0.317 & 8.428 & 0.702 & -0.314 \\
\hline Botswana & 2 & 2.166 & 0.744 & -0.332 & 2.363 & 0.742 & -0.297 & 2.936 & 0.644 & -0.266 & 2.321 & 0.719 & -0.143 & 1.611 & 0.837 & -0.212 \\
\hline DR Congo & 3 & 1.667 & 0.873 & -0.203 & 1.459 & 0.854 & -0.272 & 1.911 & 0.803 & 0.088 & 1.476 & 0.842 & & 0.736 & 0.898 & \\
\hline Lesotho & 4 & 0.789 & 0.933 & 0.106 & 0.544 & 0.896 & 0.181 & 0.794 & 0.870 & 0.355 & 0.781 & 0.907 & -0.187 & 0.457 & 0.936 & -0.250 \\
\hline Madagascar & 5 & 0.255 & 0.953 & -0.104 & 0.452 & 0.930 & -0.144 & 0.533 & 0.914 & 0.227 & 0.359 & 0.937 & 0.103 & 0.318 & 0.963 & 0.253 \\
\hline Malawi & 6 & 0.164 & 0.966 & -0.308 & 0.337 & 0.956 & -0.277 & 0.478 & 0.954 & 0.056 & 0.275 & 0.960 & -0.284 & 0.215 & 0.980 & -0.273 \\
\hline Mauritius & 7 & 0.131 & 0.976 & & 0.209 & 0.972 & & 0.242 & 0.974 & & 0.186 & 0.975 & & 0.081 & 0.987 & \\
\hline Mozambique & 8 & 0.098 & 0.983 & 0.203 & 0.119 & 0.982 & 0.160 & 0.137 & 0.985 & & 0.127 & 0.986 & & 0.066 & 0.993 & \\
\hline Namibia & 9 & 0.097 & 0.991 & 0.346 & 0.100 & 0.989 & 0.351 & 0.102 & 0.994 & 0.400 & 0.106 & 0.995 & -0.369 & 0.052 & 0.997 & -0.330 \\
\hline Seychelles & 10 & 0.067 & 0.996 & 0.331 & 0.062 & 0.994 & 0.341 & 0.058 & 0.999 & 0.391 & 0.052 & 0.999 & -0.371 & 0.029 & 0.999 & -0.331 \\
\hline South Africa & 11 & 0.039 & 0.999 & 0.292 & 0.055 & 0.998 & 0.269 & 0.013 & 1.000 & 0.226 & 0.008 & 1.000 & -0.366 & 0.005 & 1.000 & -0.321 \\
\hline Swaziland & 12 & 0.013 & 1.000 & 0.341 & 0.018 & 1.000 & 0.336 & 0.002 & 1.000 & 0.366 & 0.002 & 1.000 & -0.362 & 0.001 & 1.000 & -0.327 \\
\hline Tanzania & 13 & 0.002 & 1.000 & 0.185 & 0.003 & 1.000 & 0.221 & & & 0.246 & & & 0.168 & & & 0.306 \\
\hline Zambia & 14 & & & & & & & & & & & & -0.303 & & & -0.268 \\
\hline Zimbabwe & 15 & & & -0.323 & & & -0.299 & & & -0.163 & & & 0.305 & & & 0.246 \\
\hline No of factors & & & 3 & & & 3 & & & 3 & & & 3 & & & 2 & \\
\hline Countries excluded & \multicolumn{4}{|c|}{ MAU, ZA } & \multicolumn{3}{|c|}{ MAU, ZA } & \multicolumn{3}{|c|}{ MAU,MOZ, ZA } & \multicolumn{3}{|c|}{ DRC, MAU, MOZ } & \multicolumn{3}{|c|}{ DRC, MAU, MOZ } \\
\hline \multicolumn{17}{|l|}{ Panel B: SACU } \\
\hline Botswana & 1 & 3.833 & 0.767 & -0.431 & 3.865 & 0.773 & -0.389 & 3.439 & 0.688 & -0.187 & 3.549 & 0.710 & -0.245 & 4.309 & 0.862 & -0.412 \\
\hline Lesotho & 2 & 0.824 & 0.931 & 0.266 & 0.579 & 0.889 & 0.359 & 1.101 & 0.908 & 0.389 & 1.057 & 0.921 & & 0.484 & .959 & -0.420 \\
\hline Namibia & 3 & 0.277 & 0.987 & 0.502 & 0.486 & 0.986 & 0.497 & 0.429 & 0.994 & 0.527 & 0.373 & 0.996 & -0.522 & 0.196 & 0.998 & -0.465 \\
\hline South Africa & 4 & 0.058 & 0.998 & 0.497 & 0.065 & 0.999 & 0.492 & 0.029 & 0.999 & 0.523 & 0.018 & 0.999 & -0.523 & 0.009 & 1.000 & -0.465 \\
\hline Swaziland & 5 & 0.008 & 1.000 & 0.494 & 0.005 & 1.000 & 0.480 & 0.002 & 1.000 & 0.512 & 0.003 & 1.000 & -0.520 & 0.002 & 1.000 & -0.470 \\
\hline \multirow{2}{*}{\multicolumn{17}{|c|}{ Countries excluded }} \\
\hline & & & & & & & & & & & & & & & & \\
\hline Lesotho & 1 & 3.191 & 0.798 & -0.327 & 3.353 & 0.838 & -0.393 & 3.352 & 0.838 & -0.377 & 3.388 & 0.847 & -0.388 & 3.299 & 0.825 & -0.443 \\
\hline Namibia & 2 & 0.740 & 0.983 & -0.548 & 0.572 & 0.981 & -0.535 & 0.613 & 0.991 & -0.539 & 0.581 & 0.992 & -0.536 & 0.565 & 0.966 & -0.542 \\
\hline South Africa & 3 & 0.060 & 0.997 & -0.550 & 0.068 & 0.998 & -0.535 & 0.031 & 0.999 & -0.537 & 0.027 & 0.999 & -0.536 & 0.134 & 1.000 & -0.543 \\
\hline Swaziland & 4 & 0.010 & 1.000 & -0.539 & 0.008 & 1.000 & -0.523 & 0.004 & 1.000 & -0.529 & 0.003 & 1.000 & -0.524 & 0.002 & 1.000 & 0.464 \\
\hline $\begin{array}{l}\text { No of factors } \\
\text { Countries excluded }\end{array}$ & & & 1 & & & 1 & & & 1 & & & 1 & & & 1 & \\
\hline \multicolumn{17}{|l|}{ Panel D: OTHER } \\
\hline Angola & 1 & 4.280 & 0.535 & -0.445 & 4.271 & 0.534 & -0.396 & 2.750 & 0.393 & -0.280 & 3.954 & 0.565 & -0.441 & 5.252 & 0.750 & -0.413 \\
\hline DR Congo & 2 & 1.917 & 0.775 & -0.221 & 1.540 & 0.726 & -0.377 & 1.537 & 0.612 & 0.494 & 1.532 & 0.784 & & 0.780 & 0.862 & \\
\hline Madagas & 3 & 1.041 & 0.905 & -0.118 & 0.822 & 0.829 & -0.273 & 1.119 & 0.772 & 0.532 & 0.587 & 0.868 & 0.151 & 0.514 & 0.935 & 0.315 \\
\hline Malawi & 4 & 0.269 & 0.938 & -0.447 & 0.447 & 0.885 & -0.407 & 0.684 & 0.870 & 0.328 & 0.406 & 0.926 & -0.410 & 0.185 & 0.962 & -0.384 \\
\hline Mauritius & 5 & 0.219 & 0.966 & & 0.348 & 0.928 & & 0.426 & 0.931 & & 0.199 & 0.954 & & 0.137 & 0.981 & \\
\hline Mozam & 6 & 0.127 & 0.982 & 0.331 & 0.275 & 0.963 & 0.294 & 0.269 & 0.969 & & 0.184 & 0.980 & & 0.075 & 0.992 & \\
\hline Seychelles & 7 & 0.084 & 0.992 & 0.445 & 0.203 & 0.988 & 0.412 & 0.214 & 1.000 & 0.386 & 0.137 & 1.000 & -0.453 & 0.057 & 1.000 & -0.414 \\
\hline Tanzania & 8 & 0.064 & 1.000 & 0.200 & 0.095 & 1.000 & 0.206 & & & 0.329 & & & 0.268 & & & 0.404 \\
\hline Zambia & 9 & & & & & & & & & & & & -0.451 & & & -0.384 \\
\hline Zimbabwe & 10 & & & -0.439 & & & -0.403 & & & -0.169 & & & 0.367 & & & 0.320 \\
\hline No of factor & & & 3 & & & 2 & & & 3 & & & 2 & & & 1 & \\
\hline Countries excluded & & & MAU, ZA & & & MAU, ZA & & & MAU & & & DRC & & & DRC & \\
\hline
\end{tabular}


Table B-2: Principal components analysis of deposit rate convergence for the SADC countries for the rolling periods

\begin{tabular}{|c|c|c|c|c|c|c|c|c|c|c|c|c|c|c|c|c|c|c|c|c|c|c|}
\hline \multirow{2}{*}{$\begin{array}{l}\text { Period } \\
\text { Countries } \\
\end{array}$} & \multirow{2}{*}{$\begin{array}{r}\text { Com- } \\
\text { ponent }\end{array}$} & \multicolumn{3}{|c|}{ 1990-1994 } & \multicolumn{3}{|c|}{ 1991-1995 } & \multicolumn{3}{|c|}{ 1992-1996 } & \multicolumn{3}{|c|}{ 1993-1997 } & \multicolumn{3}{|c|}{ 1994-1998 } & \multicolumn{3}{|c|}{ 1995-1999 } & \multicolumn{3}{|c|}{ 1996-2000 } \\
\hline & & $\begin{array}{l}\text { Eigen- } \\
\text { value }\end{array}$ & Cum $\mathrm{R}^{2}$ & Vector 1 & $\begin{array}{l}\text { Eigen- } \\
\text { value }\end{array}$ & Cum $\mathrm{R}^{2}$ & Vector 1 & $\begin{array}{l}\text { Eigen- } \\
\text { value }\end{array}$ & Cum $\mathrm{R}^{2}$ & Vector 1 & $\begin{array}{l}\text { Eigen- } \\
\text { value }\end{array}$ & Cum $\mathrm{R}^{2}$ & Vector 1 & $\begin{array}{r}\text { Eigen- } \\
\text { value }\end{array}$ & Cum R ${ }^{2}$ & Vector 1 & $\begin{array}{r}\text { Eigen- } \\
\text { value }\end{array}$ & Cum $\mathrm{R}^{2}$ & Vector 1 & $\begin{array}{r}\text { Eigen- } \\
\text { value }\end{array}$ & $\operatorname{Cum~R}^{2}$ & Vector 1 \\
\hline Panel A: SADC & & & & & & & & & & & & & & & & & & & & & & \\
\hline Angola & 1 & 5.717 & 0.635 & & 5.232 & 0.476 & & 5.021 & 0.456 & & 5.389 & 0.490 & & 5.220 & 0.435 & & 5.394 & 0.385 & -0.285 & 3.586 & 0.710 & -0.099 \\
\hline Botswana & 2 & 1.742 & 0.829 & -0.280 & 3.132 & 0.760 & -0.156 & 2.700 & 0.702 & -0.323 & 2.427 & 0.711 & -0.342 & 2.407 & 0.636 & -0.329 & 4.499 & 0.707 & -0.310 & 2.023 & 0.855 & -0.063 \\
\hline DR Congo & 3 & 0.713 & 0.908 & & 1.040 & 0.855 & & 0.940 & 0.787 & & 1.625 & 0.858 & & 2.051 & 0.807 & & 1.446 & 0.810 & & 0.608 & 0.898 & \\
\hline Lesotho & 4 & 0.366 & 0.949 & 0.405 & 0.456 & 0.896 & 0.390 & 0.716 & 0.852 & 0.415 & 0.536 & 0.907 & 0.356 & 0.743 & 0.868 & 0.089 & 0.863 & 0.872 & -0.307 & 0.435 & 0.929 & -0.376 \\
\hline Madagascar & 5 & 0.195 & 0.970 & & 0.452 & 0.937 & 0.055 & 0.612 & 0.908 & -0.078 & 0.428 & 0.946 & -0.233 & 0.533 & 0.913 & -0.353 & 0.586 & 0.913 & -0.369 & 0.276 & 0.949 & -0.090 \\
\hline Malawi & 6 & 0.134 & 0.985 & -0.370 & 0.212 & 0.957 & -0.126 & 0.376 & 0.942 & 0.146 & 0.221 & 0.966 & -0.078 & 0.432 & 0.949 & -0.218 & 0.409 & 0.943 & -0.164 & 0.259 & 0.967 & 0.260 \\
\hline Mauritius & 7 & 0.074 & 0.993 & 0.337 & 0.206 & 0.975 & 0.320 & 0.246 & 0.965 & 0.283 & 0.157 & 0.980 & 0.086 & 0.224 & 0.968 & -0.268 & 0.256 & 0.961 & -0.271 & 0.213 & 0.983 & 0.050 \\
\hline Mozambique & 8 & 0.044 & 0.998 & & 0.150 & 0.989 & & 0.195 & 0.982 & & 0.107 & 0.990 & & 0.197 & 0.984 & -0.305 & 0.160 & 0.972 & -0.336 & 0.087 & 0.989 & -0.266 \\
\hline Namibia & 9 & 0.015 & 1.000 & & 0.065 & 0.995 & 0.406 & 0.123 & 0.994 & 0.377 & 0.060 & 0.995 & 0.397 & 0.069 & 0.990 & 0.379 & 0.122 & 0.981 & 0.092 & 0.058 & 0.993 & -0.344 \\
\hline Seychelles & 10 & & & 0.408 & 0.035 & 0.998 & 0.416 & 0.055 & 0.999 & 0.388 & 0.035 & 0.999 & 0.401 & 0.060 & 0.995 & 0.379 & 0.089 & 0.987 & 0.087 & 0.040 & 0.996 & -0.324 \\
\hline South Africa & 11 & & & 0.209 & 0.021 & 1.000 & 0.178 & 0.016 & 1.000 & 0.165 & 0.015 & 1.000 & 0.115 & 0.038 & 0.998 & -0.224 & 0.067 & 0.992 & -0.300 & 0.029 & 0.998 & -0.360 \\
\hline Swaziland & 12 & & & 0.240 & & & 0.367 & & & 0.351 & & & 0.403 & 0.026 & 1.000 & 0.388 & 0.049 & 0.996 & 0.140 & 0.016 & 0.999 & -0.331 \\
\hline Tanzania & 13 & & & & & & & & & & & & -0.298 & & & & 0.032 & 0.998 & -0.350 & 0.015 & 1.000 & -0.139 \\
\hline Zambia & 14 & & & -0.338 & & & -0.309 & & & -0.317 & & & -0.324 & & & -0.243 & 0.028 & 1.000 & -0.308 & & & -0.262 \\
\hline Zimbabwe & 15 & & & -0.355 & & & -0.320 & & & -0.269 & & & & & & -0.008 & & & 0.191 & & & 0.383 \\
\hline
\end{tabular}

Zimbabwe

3

Countries excluded NA, TA

\begin{tabular}{|c|c|c|c|c|c|c|c|c|c|c|c|c|c|c|c|c|c|c|c|c|c|c|}
\hline \multicolumn{23}{|l|}{ Panel B: SACU } \\
\hline Botswana & 1 & 2.719 & 0.680 & -0.395 & 3.532 & 0.706 & -0.163 & 3.730 & 0.746 & -0.300 & 4.028 & 0.806 & -0.378 & 3.528 & 0.706 & -0.367 & 2.994 & 0.599 & -0.167 & 3.721 & 0.744 & -0.127 \\
\hline Lesotho & 2 & 1.112 & 0.958 & 0.590 & 1.088 & 0.924 & 0.483 & 0.860 & 0.918 & 0.461 & 0.568 & 0.919 & 0.426 & 0.845 & 0.875 & 0.304 & 1.372 & 0.873 & 0.255 & 1.040 & 0.952 & 0.464 \\
\hline Namibia & 3 & 0.124 & 0.989 & & 0.200 & 0.964 & 0.507 & 0.263 & 0.970 & 0.488 & 0.311 & 0.981 & 0.473 & 0.460 & 0.967 & 0.513 & 0.424 & 0.958 & 0.561 & 0.142 & 0.981 & 0.512 \\
\hline South Africa & 4 & 0.044 & 1.000 & 0.594 & 0.130 & 0.990 & 0.504 & 0.096 & 0.990 & 0.491 & 0.073 & 0.996 & 0.477 & 0.130 & 0.993 & 0.499 & 0.159 & 0.990 & 0.545 & 0.074 & 0.995 & 0.501 \\
\hline Swaziland & 5 & & & 0.379 & 0.050 & 1.000 & 0.478 & 0.052 & 1.000 & 0.468 & 0.021 & 1.000 & 0.474 & 0.037 & 1.000 & 0.510 & 0.051 & 1.000 & 0.544 & 0.023 & 1.000 & 0.505 \\
\hline $\begin{array}{l}\text { No of factors } \\
\text { Countries excluded }\end{array}$ & \multicolumn{4}{|c|}{$\begin{array}{c}2 \\
\mathrm{NA}\end{array}$} & \multicolumn{3}{|c|}{2} & \multicolumn{3}{|c|}{1} & \multicolumn{3}{|c|}{1} & \multicolumn{3}{|c|}{1} & \multicolumn{3}{|c|}{2} & \multicolumn{3}{|c|}{2} \\
\hline \multicolumn{23}{|l|}{ Panel C: CMA } \\
\hline Lesotho & 1 & 2.422 & 0.807 & -0.622 & 3.465 & 0.866 & -0.475 & 3.462 & 0.866 & -0.462 & 3.523 & 0.881 & -0.447 & 3.137 & 0.784 & -0.354 & 2.940 & 0.735 & -0.295 & 3.677 & 0.919 & -0.476 \\
\hline Namibia & 2 & 0.529 & 0.984 & & 0.316 & 0.945 & -0.519 & 0.383 & 0.961 & -0.516 & 0.382 & 0.976 & -0.515 & 0.696 & 0.958 & -0.547 & 0.835 & 0.944 & -0.564 & 0.224 & 0.975 & -0.515 \\
\hline South Africa & 3 & 0.049 & 1.000 & -0.600 & 0.168 & 0.987 & -0.513 & 0.102 & 0.987 & -0.517 & 0.073 & 0.995 & -0.519 & 0.131 & 0.991 & -0.530 & 0.175 & 0.987 & -0.547 & 0.076 & 0.994 & -0.501 \\
\hline Swaziland & 4 & & & -0.503 & 0.052 & 1.000 & -0.492 & 0.052 & 1.000 & -0.503 & 0.022 & 1.000 & -0.515 & 0.037 & 1.000 & -0.543 & 0.051 & 1.000 & -0.544 & 0.023 & 1.000 & -0.507 \\
\hline $\begin{array}{l}\text { No of factors } \\
\text { Countries excluded }\end{array}$ & & & $\begin{array}{c}1 \\
\mathrm{NA}\end{array}$ & & & 1 & & & 1 & & & 1 & & & 1 & & & 1 & & & 1 & \\
\hline \multicolumn{23}{|l|}{ Panel D: OTHER } \\
\hline Angola & 1 & 3.100 & 0.620 & & 2.319 & 0.386 & & 2.510 & 0.418 & & 2.059 & 0.343 & & 3.062 & 0.437 & & 4.270 & 0.474 & -0.325 & 3.977 & 0.442 & -0.206 \\
\hline DR Congo & 2 & 1.210 & 0.862 & & 2.036 & 0.726 & & 1.279 & 0.632 & & 1.977 & 0.673 & & 2.171 & 0.748 & & 2.740 & 0.779 & & 2.693 & 0.741 & \\
\hline Madagascar & 3 & 0.404 & 0.943 & & 0.840 & 0.866 & -0.422 & 0.881 & 0.778 & -0.421 & 1.085 & 0.854 & -0.523 & 0.759 & 0.856 & -0.535 & 0.808 & 0.869 & -0.419 & 0.980 & 0.850 & -0.308 \\
\hline Malawi & 4 & 0.180 & 0.979 & -0.484 & 0.342 & 0.923 & 0.310 & 0.619 & 0.882 & 0.422 & 0.487 & 0.935 & -0.574 & 0.538 & 0.933 & -0.291 & 0.558 & 0.931 & -0.161 & 0.593 & 0.916 & 0.252 \\
\hline Mauritius & 5 & 0.105 & 1.000 & 0.479 & 0.280 & 0.969 & 0.554 & 0.484 & 0.962 & 0.544 & 0.271 & 0.980 & -0.322 & 0.237 & 0.967 & -0.347 & 0.228 & 0.956 & -0.275 & 0.279 & 0.947 & -0.013 \\
\hline Mozambique & 6 & & & & 0.184 & 1.000 & & 0.226 & 1.000 & & 0.120 & 1.000 & & 0.151 & 0.988 & -0.410 & 0.157 & 0.974 & -0.378 & 0.264 & 0.976 & -0.398 \\
\hline Seychelles & 7 & & & 0.257 & & & -0.258 & & & -0.211 & & & -0.175 & 0.081 & 1.000 & -0.421 & 0.141 & 0.989 & -0.359 & 0.151 & 0.993 & -0.454 \\
\hline Tanzania & 8 & & & & & & & & & & & & & & & & 0.067 & 0.997 & -0.379 & 0.046 & 0.998 & -0.248 \\
\hline Zambia & 9 & & & -0.483 & & & -0.527 & & & -0.494 & & & -0.129 & & & -0.361 & 0.030 & 1.000 & -0.370 & 0.017 & 1.000 & -0.450 \\
\hline Zimbabwe & 10 & & & -0.488 & & & -0.274 & & & -0.245 & & & -0.496 & & & 0.183 & & & 0.254 & & & 0.413 \\
\hline No of factors & \multirow{2}{*}{\multicolumn{4}{|c|}{$\begin{array}{c}2 \\
\text { ANG, DRC, MAD, MOZ, } \\
\text { TA }\end{array}$}} & \multirow{2}{*}{\multicolumn{3}{|c|}{$\begin{array}{c}2 \\
\text { ANG, DRC, } M\end{array}$}} & \multirow{2}{*}{\multicolumn{3}{|c|}{2}} & \multirow{2}{*}{\multicolumn{3}{|c|}{$\begin{array}{l}3 \\
\text { RC, MOZ, TA }\end{array}$}} & \multicolumn{3}{|c|}{$\begin{array}{l}2 \\
\text { DRC, TA }\end{array}$} & \multirow{2}{*}{\multicolumn{3}{|c|}{$\begin{array}{c}2 \\
\text { DRC }\end{array}$}} & \multirow{2}{*}{\multicolumn{3}{|c|}{$\begin{array}{c}2 \\
\text { DRC }\end{array}$}} \\
\hline Countries excluded & & & & & & & & & & & & & & ANC & DRC, & & & & & & & \\
\hline
\end{tabular}


Table B-2 continued: Principal components analysis of deposit rate convergence for the SADC countries for the rolling periods

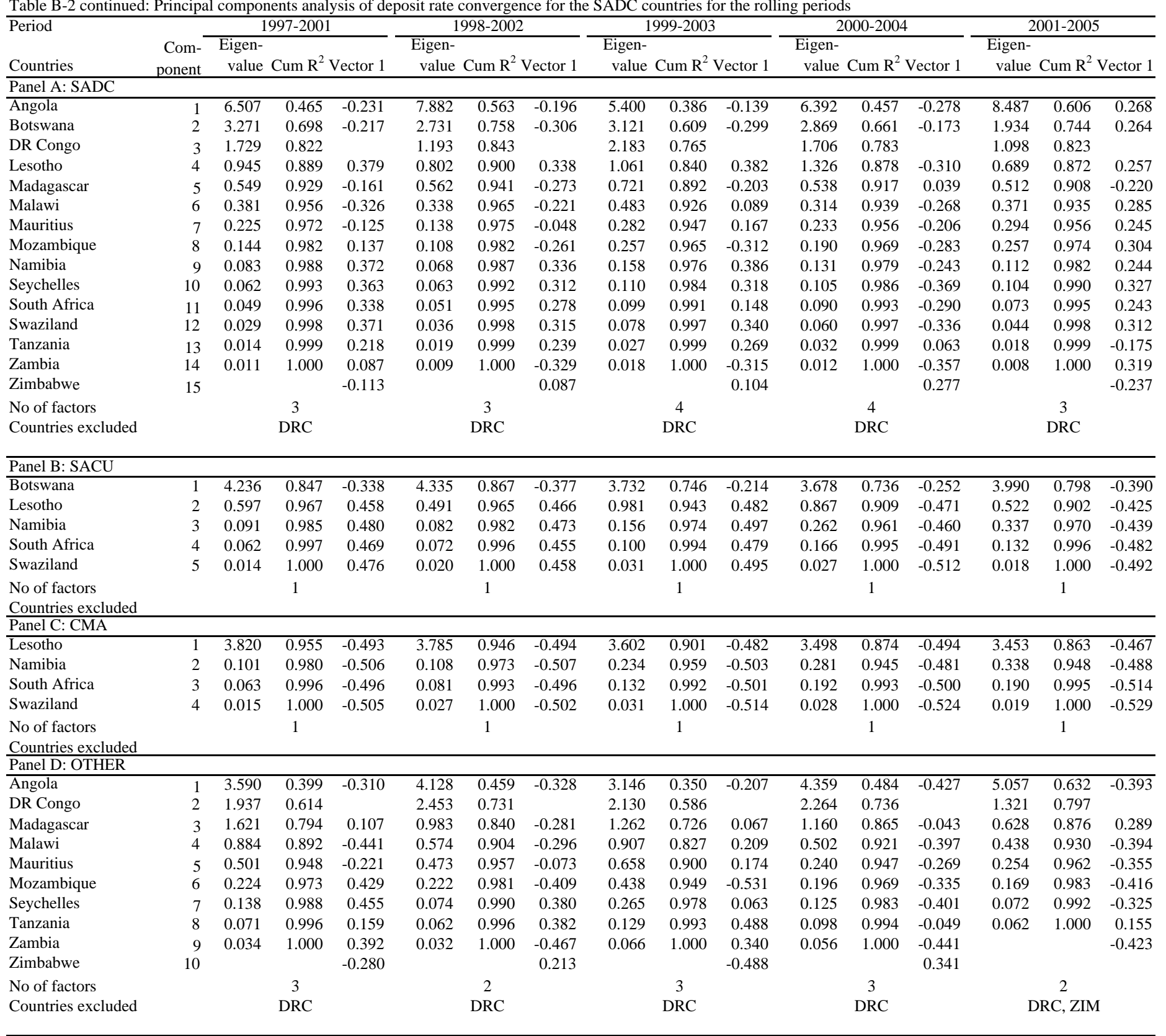


Table B-3: Principal components analysis of lending rates convergence for the SADC Countries for the rolling period:

Table B-3: Principal components analysis of lending rates convergence for the SADC Countries for the rolling period:

\begin{tabular}{|c|c|c|c|c|c|c|c|c|c|c|c|c|c|c|c|c|c|c|c|c|c|c|}
\hline \multirow{2}{*}{$\begin{array}{l}\text { Period } \\
\text { Countries } \\
\end{array}$} & \multicolumn{4}{|c|}{ 1990-1994 } & \multicolumn{3}{|c|}{ 1991-1995 } & \multicolumn{3}{|c|}{$1992-1996$} & \multicolumn{3}{|c|}{ 1993-1997 } & \multicolumn{3}{|c|}{ 1994-1998 } & \multicolumn{3}{|c|}{ 1995-1999 } & \multicolumn{3}{|c|}{ 1996-2000 } \\
\hline & $\begin{array}{r}\text { Com- } \\
\text { ponent }\end{array}$ & $\begin{array}{l}\text { Eigen- } \\
\text { value }\end{array}$ & Cum $\mathrm{R}^{2}$ & Vector 1 & $\begin{array}{r}\text { Eigen- } \\
\text { value }\end{array}$ & Cum $\mathrm{R}^{2}$ & Vector 1 & $\begin{array}{l}\text { Eigen- } \\
\text { value }\end{array}$ & Cum $\mathrm{R}^{2}$ & Vector 1 & $\begin{array}{r}\text { Eigen- } \\
\text { value }\end{array}$ & Cum $\mathrm{R}^{2}$ & Vector 1 & $\begin{array}{r}\text { Eigen- } \\
\text { value }\end{array}$ & Cum $\mathrm{R}^{2}$ & Vector 1 & $\begin{array}{l}\text { Eigen- } \\
\text { value }\end{array}$ & Cum $\mathrm{R}^{2}$ & Vector 1 & $\begin{array}{l}\text { Eigen- } \\
\text { value }\end{array}$ & Cum $R^{2}$ & Vector 1 \\
\hline Panel A: SADC & & & & & & & & & & & & & & & & & & & & & & \\
\hline Angola & 1 & 5.646 & 0.627 & & 5.703 & 0.518 & & 4.724 & 0.429 & & 5.025 & 0.419 & & 6.238 & 0.480 & & 5.553 & 0.397 & -0.288 & 6.078 & 0.434 & -0.021 \\
\hline Botswana & 2 & 1.508 & 0.795 & -0.302 & 3.303 & 0.819 & -0.333 & 3.156 & 0.716 & -0.012 & 3.065 & 0.674 & 0.137 & 3.205 & 0.726 & -0.095 & 3.562 & 0.651 & -0.201 & 4.107 & 0.727 & -0.331 \\
\hline DR Congo & 3 & 1.118 & 0.919 & & 0.733 & 0.885 & & 1.374 & 0.841 & & 1.608 & 0.808 & & 1.162 & 0.816 & -0.357 & 2.155 & 0.805 & -0.373 & 1.640 & 0.845 & -0.039 \\
\hline Lesotho & 4 & 0.314 & 0.954 & 0.379 & 0.532 & 0.934 & 0.372 & 0.609 & 0.897 & -0.236 & 0.693 & 0.866 & -0.159 & 0.949 & 0.889 & 0.368 & 0.921 & 0.871 & 0.328 & 0.827 & 0.904 & 0.139 \\
\hline Madagascar & 5 & 0.187 & 0.975 & & 0.271 & 0.958 & -0.194 & 0.398 & 0.933 & -0.392 & 0.571 & 0.913 & 0.339 & 0.428 & 0.922 & -0.132 & 0.639 & 0.916 & -0.353 & 0.670 & 0.952 & 0.212 \\
\hline Malawi & 6 & 0.130 & 0.989 & -0.397 & 0.196 & 0.976 & -0.276 & 0.332 & 0.963 & -0.326 & 0.347 & 0.942 & 0.443 & 0.350 & 0.949 & -0.008 & 0.343 & 0.941 & -0.153 & 0.170 & 0.964 & -0.292 \\
\hline Mauritius & 7 & 0.052 & 0.995 & 0.057 & 0.112 & 0.986 & -0.104 & 0.194 & 0.981 & -0.366 & 0.260 & 0.964 & 0.332 & 0.274 & 0.970 & 0.012 & 0.262 & 0.960 & -0.105 & 0.150 & 0.974 & -0.151 \\
\hline Mozambique & 8 & 0.027 & 0.998 & & 0.051 & 0.991 & & 0.104 & 0.990 & & 0.177 & 0.979 & & 0.123 & 0.979 & & 0.188 & 0.973 & & 0.121 & 0.983 & \\
\hline Namibia & 9 & 0.018 & 1.000 & & 0.048 & 0.995 & 0.387 & 0.058 & 0.995 & -0.200 & 0.117 & 0.989 & -0.128 & 0.103 & 0.987 & 0.356 & 0.123 & 0.982 & 0.263 & 0.084 & 0.989 & 0.350 \\
\hline Seychelles & 10 & & & 0.409 & 0.026 & 0.998 & 0.377 & 0.031 & 0.998 & -0.368 & 0.090 & 0.996 & -0.223 & 0.078 & 0.993 & 0.369 & 0.103 & 0.989 & 0.282 & 0.059 & 0.993 & 0.345 \\
\hline South Africa & 11 & & & -0.292 & 0.025 & 1.000 & -0.331 & 0.021 & 1.000 & -0.283 & 0.031 & 0.999 & 0.411 & 0.046 & 0.997 & -0.299 & 0.063 & 0.994 & -0.204 & 0.036 & 0.996 & 0.351 \\
\hline Swaziland & 12 & & & 0.268 & & & 0.151 & & & -0.440 & 0.015 & 1.000 & -0.112 & 0.029 & 0.999 & 0.342 & 0.039 & 0.996 & 0.249 & 0.026 & 0.998 & 0.371 \\
\hline Tanzania & 13 & & & & & & & & & & & & 0.490 & 0.013 & 1.000 & -0.335 & 0.031 & 0.999 & -0.359 & 0.021 & 0.999 & 0.216 \\
\hline Zambia & 14 & & & -0.346 & & & -0.211 & & & 0.317 & & & -0.118 & & & -0.262 & 0.019 & 1.000 & -0.268 & 0.012 & 1.000 & 0.127 \\
\hline Zimbabwe & 15 & & & -0.400 & & & -0.398 & & & -0.014 & & & 0.181 & & & 0.246 & & & 0.132 & & & -0.389 \\
\hline
\end{tabular}

Zimbabwe

ANG, MOZ

$\mathrm{MOZ}$

$\mathrm{MOZ}$

\begin{tabular}{|c|c|c|c|c|c|c|c|c|c|c|c|c|c|c|c|c|c|c|c|c|c|c|}
\hline \multirow{2}{*}{\multicolumn{23}{|c|}{ Panel B: SACU }} \\
\hline & & & & & & & & & & & & & & & & & & & & & & \\
\hline Botswana & 1 & 2.775 & 0.694 & -0.470 & 3.659 & 0.732 & -0.407 & 3.100 & 0.620 & -0.059 & 3.442 & 0.688 & -0.052 & 3.702 & 0.740 & -0.043 & 3.473 & 0.695 & -0.214 & 3.928 & 0.786 & -0.387 \\
\hline Lesotho & 2 & 1.040 & 0.954 & 0.568 & 0.884 & 0.909 & 0.474 & 1.039 & 0.828 & 0.488 & 1.044 & 0.897 & 0.463 & 0.999 & 0.940 & -0.504 & 0.986 & 0.892 & 0.433 & 0.798 & 0.945 & 0.362 \\
\hline Namibia & 3 & 0.157 & 0.993 & & 0.284 & 0.966 & 0.495 & 0.493 & 0.926 & 0.479 & 0.327 & 0.963 & 0.482 & 0.155 & 0.971 & -0.493 & 0.327 & 0.957 & 0.502 & 0.177 & 0.981 & 0.489 \\
\hline South Africa & 4 & 0.028 & 1.000 & 0.592 & 0.132 & 0.992 & 0.510 & 0.325 & 0.991 & 0.555 & 0.167 & 0.996 & 0.527 & 0.127 & 0.997 & -0.510 & 0.146 & 0.986 & 0.520 & 0.068 & 0.994 & 0.495 \\
\hline Swaziland & 5 & & & 0.326 & 0.040 & 1.000 & 0.323 & 0.043 & 1.000 & 0.470 & 0.020 & 1.000 & 0.522 & 0.017 & 1.000 & -0.491 & 0.069 & 1.000 & 0.494 & 0.030 & 1.000 & 0.485 \\
\hline $\begin{array}{l}\text { No of factors } \\
\text { Countries excluded }\end{array}$ & \multicolumn{4}{|c|}{$\begin{array}{c}2 \\
\text { NA } \\
\end{array}$} & \multicolumn{3}{|c|}{1} & \multicolumn{3}{|c|}{2} & \multicolumn{3}{|c|}{2} & \multicolumn{3}{|c|}{2} & \multicolumn{3}{|c|}{1} & \multicolumn{3}{|c|}{1} \\
\hline \multicolumn{23}{|l|}{ Panel C: CMA } \\
\hline Lesotho & 1 & 2.301 & 0.767 & -0.612 & 3.137 & 0.784 & -0.513 & 3.093 & 0.773 & -0.488 & 3.436 & 0.859 & -0.467 & 3.697 & 0.924 & -0.504 & 3.355 & 0.839 & -0.459 & 3.416 & 0.854 & -0.430 \\
\hline Namibia & 2 & 0.594 & 0.965 & & 0.641 & 0.944 & -0.517 & 0.528 & 0.905 & -0.479 & 0.347 & 0.946 & -0.483 & 0.156 & 0.963 & -0.494 & 0.418 & 0.943 & -0.510 & 0.482 & 0.975 & -0.520 \\
\hline South Africa & 3 & 0.105 & 1.000 & -0.622 & 0.181 & 0.990 & -0.556 & 0.326 & 0.987 & -0.555 & 0.196 & 0.995 & -0.527 & 0.129 & 0.996 & -0.511 & 0.146 & 0.980 & -0.528 & 0.068 & 0.992 & -0.532 \\
\hline Swaziland & 4 & & & -0.489 & 0.041 & 1.000 & -0.400 & 0.054 & 1.000 & -0.474 & 0.021 & 1.000 & -0.521 & 0.018 & 1.000 & -0.491 & 0.081 & 1.000 & -0.500 & 0.034 & 1.000 & -0.511 \\
\hline $\begin{array}{l}\text { No of factors } \\
\text { Countries excluded }\end{array}$ & \multicolumn{4}{|c|}{1} & \multicolumn{3}{|c|}{1} & \multicolumn{3}{|c|}{1} & \multicolumn{3}{|c|}{1} & \multicolumn{3}{|c|}{1} & \multicolumn{3}{|c|}{1} & \multicolumn{3}{|c|}{1} \\
\hline \multicolumn{23}{|l|}{ Panel D: OTHER } \\
\hline Angola & 1 & 3.057 & 0.611 & & 3.446 & 0.574 & & 3.541 & 0.590 & & 3.410 & 0.487 & & 3.356 & 0.420 & & 4.470 & 0.497 & -0.318 & 4.454 & 0.495 & -0.218 \\
\hline DR Congo & 2 & 1.228 & 0.857 & & 1.853 & 0.883 & & 1.406 & 0.825 & & 1.743 & 0.736 & & 2.379 & 0.717 & -0.505 & 2.344 & 0.757 & -0.385 & 2.594 & 0.783 & -0.297 \\
\hline Madagascar & 3 & 0.555 & 0.968 & & 0.346 & 0.941 & -0.500 & 0.618 & 0.928 & -0.518 & 0.663 & 0.831 & -0.507 & 0.997 & 0.841 & -0.247 & 0.720 & 0.837 & -0.455 & 0.817 & 0.874 & -0.455 \\
\hline Malawi & 4 & 0.114 & 0.991 & -0.529 & 0.257 & 0.984 & -0.509 & 0.335 & 0.983 & -0.475 & 0.574 & 0.913 & -0.472 & 0.703 & 0.929 & -0.099 & 0.625 & 0.907 & -0.073 & 0.702 & 0.952 & 0.156 \\
\hline Mauritius & 5 & 0.047 & 1.000 & 0.124 & 0.067 & 0.995 & -0.409 & 0.071 & 0.995 & -0.486 & 0.395 & 0.969 & -0.459 & 0.289 & 0.966 & 0.005 & 0.383 & 0.949 & -0.037 & 0.156 & 0.969 & 0.081 \\
\hline Mozambique & 6 & & & & 0.030 & 1.000 & & 0.029 & 1.000 & & 0.175 & 0.994 & & 0.147 & 0.984 & & 0.253 & 0.977 & & 0.126 & 0.983 & \\
\hline Seychelles & 7 & & & -0.424 & & & -0.412 & & & -0.345 & 0.040 & 1.000 & -0.233 & 0.083 & 0.994 & 0.415 & 0.110 & 0.989 & -0.362 & 0.074 & 0.991 & -0.394 \\
\hline Tanzania & 8 & & & & & & & & & & & & -0.340 & 0.046 & 1.000 & -0.495 & 0.066 & 0.997 & -0.432 & 0.056 & 0.998 & -0.447 \\
\hline Zambia & 9 & & & -0.498 & & & 0.040 & & & 0.284 & & & 0.368 & & & -0.388 & 0.029 & 1.000 & -0.346 & 0.022 & 1.000 & -0.378 \\
\hline Zimbabwe & 10 & & & -0.526 & & & -0.391 & & & -0.264 & & & 0.060 & & & 0.326 & & & 0.314 & & & 0.358 \\
\hline No of factors & \multirow{2}{*}{\multicolumn{4}{|c|}{$\begin{array}{c}2 \\
\text { ANG, DRC, MAD, MOZ, }\end{array}$}} & \multicolumn{3}{|c|}{2} & \multirow{2}{*}{\multicolumn{3}{|c|}{$\begin{array}{c}2 \\
\text { ANG, DRC, MOZ, TA }\end{array}$}} & \multirow{2}{*}{\multicolumn{3}{|c|}{$\begin{array}{c}2 \\
\text { ANG, DRC, MOZ, }\end{array}$}} & \multirow{2}{*}{\multicolumn{3}{|c|}{$\begin{array}{c}3 \\
\text { ANG, MOZ, }\end{array}$}} & \multirow{2}{*}{\multicolumn{3}{|c|}{$\stackrel{2}{\mathrm{MOZ}}$}} & \multirow{2}{*}{\multicolumn{3}{|c|}{$\stackrel{2}{\mathrm{MOZ}}$}} \\
\hline Countries excluded & & & & & ANG, I & $\mathrm{RC}, \mathrm{MO}$ & $\mathrm{Z}, \mathrm{TA}$ & & & & & & & & & & & & & & & \\
\hline
\end{tabular}


Table B-3 continued: Principal components analysis of lending rates convergence for the SADC Countries for the rolling period:

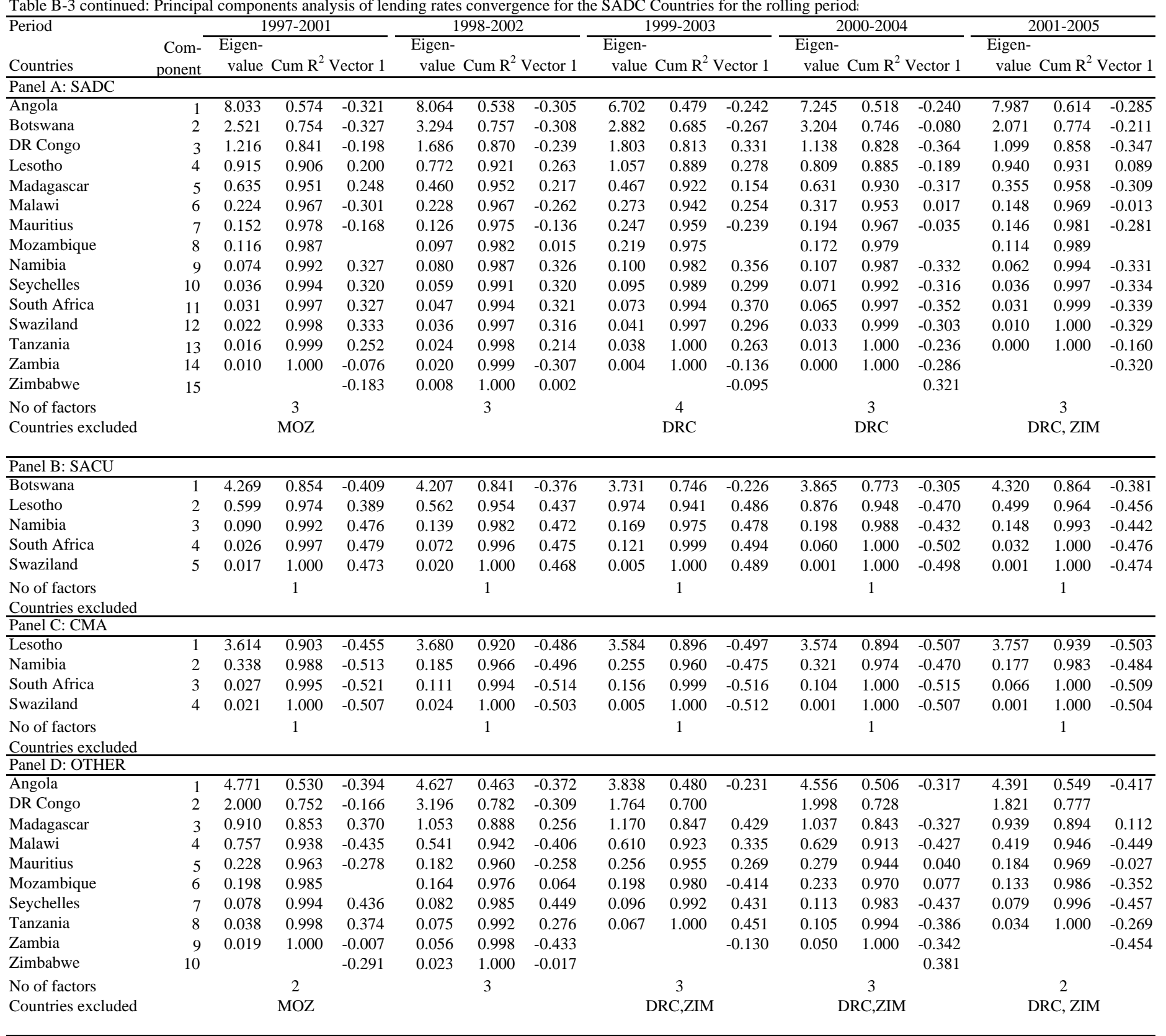


Table B-4: Unit root tests which allow for the presense of a structural breal

\begin{tabular}{|c|c|c|c|c|c|c|c|c|c|c|c|c|c|c|c|}
\hline \multirow[b]{2}{*}{ Country } & \multicolumn{6}{|c|}{ Mean shift } & \multicolumn{6}{|c|}{$\begin{array}{c}\text { Trend shift } \\
\end{array}$} & \multicolumn{3}{|c|}{ Recursive } \\
\hline & $\min \mathrm{t}($ level) & $\min \mathrm{t}($ diff $)$ & $\mathrm{I}(?)$ & max F(level) & $\max F$ (diff) & $\mathrm{I}(?)$ & min t(level) & $\min t($ diff $)$ & $\mathrm{I}(?)$ & max F(level) & max F(diff) & $\mathrm{I}(?)$ & $\min \mathrm{t}($ level) & $\min t($ diff $)$ & $\overline{I(?)}$ \\
\hline \multicolumn{16}{|c|}{ Panel A: Bank rate } \\
\hline Angola & -3.05 & -6.98 & 1 & 7.03 & 62.55 & 1 & -2.98 & -7.02 & 1 & 6.72 & 62.55 & 1 & -2.15 & -7.91 & 1 \\
\hline Botswana & -3.20 & -9.40 & 1 & 10.20 & 64.57 & 1 & -3.20 & -9.40 & 1 & 9.46 & 63.29 & 1 & -3.68 & -10.54 & 0 \\
\hline DR Congo & -2.55 & -8.11 & 1 & 11.04 & 48.43 & 1 & -2.52 & -8.11 & 1 & 14.76 & 46.23 & 1 & -2.06 & -9.07 & 1 \\
\hline Lesotho & -2.64 & -8.83 & 1 & 8.15 & 53.78 & 1 & -2.62 & -8.83 & 1 & 7.73 & 53.90 & 1 & -2.48 & -9.94 & 1 \\
\hline Madagascar & -1.43 & -7.07 & 1 & 10.19 & 40.43 & 1 & -1.97 & -7.07 & 1 & 13.85 & 45.80 & 1 & -1.52 & -7.74 & 1 \\
\hline Malawi & -2.46 & -8.55 & 1 & 8.25 & 53.29 & 1 & -2.44 & -8.55 & 1 & 8.48 & 51.68 & 1 & -1.87 & -9.62 & 1 \\
\hline Mauritius & -2.36 & -3.78 & 2 & 16.29 & 16.12 & 2 & -1.96 & -3.78 & 2 & 7.42 & 16.04 & 2 & -2.35 & -4.88 & 1 \\
\hline Mozambique & -4.19 & -6.31 & 0 & 33.86 & 49.50 & 0 & -1.98 & -6.33 & 1 & 13.14 & 49.50 & 1 & -1.18 & -7.04 & 1 \\
\hline Namibia & -2.03 & -5.41 & 1 & 10.76 & 36.14 & 1 & -2.66 & -5.40 & 1 & 10.81 & 36.14 & 1 & -1.91 & -6.01 & 1 \\
\hline Seychelles & -3.85 & -8.88 & 0 & 25.64 & 55.04 & 0 & -2.02 & -8.88 & 1 & 4.34 & 56.97 & 1 & -3.36 & -9.97 & 0 \\
\hline South Africa & -2.39 & -6.28 & 1 & 7.97 & 31.80 & 1 & -2.36 & -6.28 & 1 & 8.89 & 30.41 & 1 & -1.97 & -7.06 & 1 \\
\hline Swaziland & -2.22 & -6.58 & 1 & 7.38 & 30.88 & 1 & -2.15 & -6.58 & 1 & 8.99 & 29.74 & 1 & -1.26 & -7.38 & 1 \\
\hline Tanzania & -2.56 & -7.23 & 1 & 8.95 & 65.67 & 1 & -2.60 & -7.24 & 1 & 10.22 & 65.67 & 1 & -2.87 & -8.10 & 1 \\
\hline Zambia & -2.91 & -5.65 & 1 & 13.28 & 39.22 & 1 & -2.90 & -5.65 & 0 & 17.61 & 39.22 & 0 & -3.20 & -6.26 & 0 \\
\hline Zimbabwe & -2.17 & -6.59 & 1 & 5.87 & 28.86 & 1 & -2.26 & -6.62 & 1 & 6.43 & 29.14 & 1 & -3.78 & -8.67 & 0 \\
\hline \multicolumn{16}{|c|}{ Panel B: Deposit rate } \\
\hline Angola & -4.75 & $\begin{array}{l}-7.14 \\
\end{array}$ & 0 & 35.92 & 65.16 & 0 & -9.17 & -7.14 & 0 & 123.23 & 65.16 & 0 & -3.22 & -8.07 & 0 \\
\hline Botswana & -2.85 & -10.37 & 1 & 11.82 & 76.01 & 1 & -2.85 & -10.37 & 1 & 12.02 & 75.74 & 1 & -3.21 & -11.67 & 0 \\
\hline DR Congo & na & na & na & na & na & na & na & na & na & na & na & na & na & na & na \\
\hline Lesotho & -2.08 & -9.24 & 1 & 8.13 & 57.01 & 1 & -1.87 & -9.24 & 1 & 5.75 & 58.34 & 1 & -1.97 & -10.39 & 1 \\
\hline Madagascar & -2.13 & -8.08 & 1 & 10.00 & 44.80 & 1 & -2.21 & -8.09 & 1 & 0.01 & 43.26 & 1 & -2.51 & -9.01 & 1 \\
\hline Malawi & -2.28 & -8.41 & 1 & 4.51 & 47.99 & 1 & -2.25 & -8.41 & 1 & 4.69 & 48.92 & 1 & -1.69 & -9.47 & 1 \\
\hline Mauritius & -3.02 & -9.52 & 1 & 7.16 & 58.93 & 1 & -3.00 & -9.52 & 1 & 7.30 & 61.41 & 1 & -3.08 & -10.71 & 0 \\
\hline Mozambique & -3.15 & -7.18 & 1 & 11.84 & 64.98 & 1 & -3.60 & -7.17 & 1 & 8.04 & 64.98 & 1 & -2.66 & -8.06 & 1 \\
\hline Namibia & -2.09 & -6.68 & 1 & 11.02 & 35.13 & 1 & -2.43 & -6.67 & 1 & 11.16 & 33.00 & 1 & -1.65 & -7.39 & 1 \\
\hline Seychelles & -2.87 & -9.29 & 1 & 14.61 & 58.97 & 1 & -2.76 & -9.29 & 1 & 11.88 & 57.97 & 1 & -2.30 & -10.42 & 1 \\
\hline South Africa & -2.36 & -6.11 & 1 & 12.98 & 34.74 & 1 & -2.34 & -6.11 & 1 & 6.98 & 32.04 & 1 & -2.33 & -6.87 & 1 \\
\hline Swaziland & -2.12 & -6.78 & 1 & 7.89 & 32.40 & 1 & -2.10 & -6.78 & 1 & 8.51 & 31.21 & 1 & -1.70 & -7.62 & 1 \\
\hline Tanzania & -2.73 & -5.97 & 0 & 21.80 & 44.18 & 0 & -3.45 & -5.97 & 0 & 30.44 & 44.18 & 0 & -2.09 & -6.65 & 1 \\
\hline Zambia & -2.63 & -5.92 & 1 & 10.25 & 31.25 & 1 & -2.63 & -5.92 & 1 & 10.83 & 35.73 & 1 & -2.99 & -6.65 & 0 \\
\hline Zimbabwe & -4.96 & -11.26 & 1 & 18.14 & 83.21 & 1 & -4.87 & -11.25 & 1 & 13.33 & 83.09 & 1 & -3.90 & -12.70 & 0 \\
\hline \multicolumn{16}{|c|}{ Panel C: Lending rate } \\
\hline Angola & -5.10 & $\begin{array}{l}-7.92 \\
\end{array}$ & 0 & 40.38 & 80.40 & 0 & -7.57 & $\begin{array}{l}-7.92 \\
\end{array}$ & 0 & 84.61 & 80.40 & 0 & -3.39 & -8.97 & 0 \\
\hline Botswana & -2.72 & -10.10 & 1 & 5.61 & 71.92 & 1 & -2.70 & -10.10 & 1 & 6.94 & 73.70 & 1 & -3.04 & -11.34 & 0 \\
\hline DR Congo & -1.78 & -6.50 & 1 & 4.80 & 46.65 & 1 & -2.44 & -6.33 & 1 & 5.67 & 46.65 & 1 & -1.70 & -6.83 & 1 \\
\hline Lesotho & -2.89 & -11.14 & 1 & 6.90 & 84.27 & 1 & -2.86 & -11.14 & 1 & 6.33 & 84.50 & 1 & -2.35 & -12.54 & 1 \\
\hline Madagascar & -1.15 & -7.60 & 1 & 11.87 & 48.43 & 1 & -2.05 & -7.61 & 1 & 15.92 & 51.12 & 1 & -1.41 & -8.38 & 1 \\
\hline Malawi & -2.58 & -8.07 & 1 & 5.29 & 44.16 & 1 & -2.56 & -8.06 & 1 & 5.47 & 45.52 & 1 & -1.92 & -9.07 & 1 \\
\hline Mauritius & -3.57 & -8.11 & 0 & 19.19 & 44.66 & 0 & -3.57 & -8.11 & 1 & 15.18 & 43.79 & 1 & -3.87 & -9.14 & 0 \\
\hline Mozambique & -3.04 & -6.12 & 1 & 11.65 & 47.24 & 1 & -3.04 & -6.12 & 1 & 0.01 & 47.24 & 1 & -3.41 & -6.87 & 0 \\
\hline Namibia & -2.34 & -9.96 & 1 & 8.68 & 70.98 & 1 & -2.34 & -9.94 & 1 & 9.38 & 70.19 & 1 & -2.07 & -11.05 & 1 \\
\hline Seychelles & -3.67 & -9.65 & 0 & 24.67 & 65.82 & 0 & -3.88 & -9.65 & 0 & 25.91 & 62.46 & 0 & -2.09 & -10.76 & 1 \\
\hline South Africa & -2.38 & -5.87 & 1 & 8.54 & 28.88 & 1 & -2.36 & -5.87 & 1 & 9.07 & 27.21 & 1 & -2.07 & -6.60 & 1 \\
\hline Swaziland & -2.11 & -6.51 & 1 & 7.96 & 30.25 & 1 & -2.20 & -6.51 & 1 & 9.55 & 28.99 & 1 & -1.33 & -7.31 & 1 \\
\hline Tanzania & -1.87 & -7.60 & 1 & 14.23 & 71.69 & 1 & -2.77 & -7.62 & 1 & 15.59 & 71.69 & 1 & -2.13 & -8.47 & 1 \\
\hline Zambia & -3.12 & -7.44 & 1 & 8.80 & 44.60 & 1 & -3.12 & -7.44 & 1 & 9.28 & 51.01 & 1 & -3.53 & -8.37 & 0 \\
\hline Zimbabwe & -4.95 & -7.51 & 1 & 17.13 & 38.83 & 1 & -4.81 & -7.48 & 1 & 14.72 & 38.02 & 1 & -3.81 & -11.21 & 0 \\
\hline
\end{tabular}

Note: The unit root characteristic is evaluated at the $5 \%$ significance level based on the following critical values: mean shift $\mathrm{t}=-4.80$, mean shift $\mathrm{F}=18.62$, trend shift $\mathrm{ft}=-4.48$, trend shift $\mathrm{F}=16.3 \mathrm{C}$ recursive $t=-2.88$. For details on the methodology see Banerjee, Lumsdaine, and Stock (1992). For each interest rate series, the tests are based on all available observations between January 1990 and December 2006. na indicates that series is missing. 
Rolling period from January to December of the years

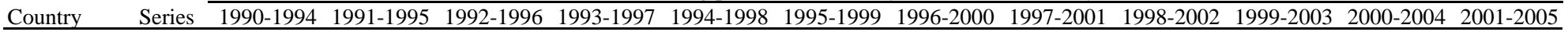
Panel A: Bank rate

\begin{tabular}{|c|c|c|c|c|c|c|c|c|c|c|c|c|c|}
\hline Angola & $\begin{array}{l}\text { Level } \\
1^{\text {st }} \text { Diff }\end{array}$ & $\begin{array}{l}\text { na } \\
\text { na }\end{array}$ & $\begin{array}{l}\text { na } \\
\text { na }\end{array}$ & $\begin{array}{l}\text { na } \\
\text { na }\end{array}$ & $\begin{array}{l}\text { na } \\
\text { na }\end{array}$ & $\begin{array}{l}\text { na } \\
\text { na }\end{array}$ & $\begin{array}{r}-1.71 \\
-7.48 a\end{array}$ & $\begin{array}{r}-1.87 \\
-7.48 a\end{array}$ & $\begin{array}{r}-1.61 \\
-7.93 a\end{array}$ & $\begin{array}{r}-1.48 \\
-7.78 a\end{array}$ & $\begin{array}{l}-3.08 b \\
-7.72 a\end{array}$ & $\begin{array}{r}-0.91 \\
-7.50 \mathrm{a}\end{array}$ & $\begin{array}{r}0.75 \\
-7.62 a\end{array}$ \\
\hline Botswana & $\begin{array}{l}\text { Level } \\
1^{\text {st }} \text { Diff }\end{array}$ & $\begin{array}{l}-4.85 a \\
-7.82 a\end{array}$ & $\begin{array}{r}-1.86 \\
-7.34 a\end{array}$ & $\begin{array}{l}-2.86 c \\
-7.34 a\end{array}$ & $\begin{array}{r}-1.33 \\
-7.40 \mathrm{a}\end{array}$ & $\begin{array}{r}-1.6 \\
-9.97 a\end{array}$ & $\begin{array}{r}-1.49 \\
-5.77 a\end{array}$ & $\begin{array}{r}-0.81 \\
-11.0 \mathrm{a}\end{array}$ & $\begin{array}{r}-0.91 \\
-11.2 \mathrm{a}\end{array}$ & $\begin{array}{r}-1.36 \\
-11.1 \mathrm{a}\end{array}$ & $\begin{array}{r}-1.74 \\
-11.1 \mathrm{a}\end{array}$ & $\begin{array}{r}-2.02 \\
-11.2 \mathrm{a}\end{array}$ & $\begin{array}{r}-1.39 \\
-6.05 a\end{array}$ \\
\hline DRC & $\begin{array}{l}\text { Level } \\
1^{\text {st }} \text { Diff }\end{array}$ & $\begin{array}{r}0.47 \\
-0.41\end{array}$ & $\begin{array}{r}-0.89 \\
-7.63 a\end{array}$ & $\begin{array}{r}-0.37 \\
-7.23 a\end{array}$ & $\begin{array}{r}-1.26 \\
-6.25 a\end{array}$ & $\begin{array}{r}-0.95 \\
-6.25 a\end{array}$ & $\begin{array}{r}-1.33 \\
-6.32 a\end{array}$ & $\begin{array}{r}-1.51 \\
-6.38 a\end{array}$ & $\begin{array}{l}-4.03 a \\
-5.96 a\end{array}$ & $\begin{array}{r}-1.29 \\
-7.44 a\end{array}$ & $\begin{array}{r}-0.75 \\
-2.96 b\end{array}$ & $\begin{array}{r}-0.61 \\
-6.98 a\end{array}$ & $\begin{array}{r}-0.89 \\
-6.06 a\end{array}$ \\
\hline Lesotho & $\begin{array}{l}\text { Level } \\
1^{\text {st }} \text { Diff }\end{array}$ & $\begin{array}{r}-1.1 \\
-7.11 \mathrm{a}\end{array}$ & $\begin{array}{r}-1.19 \\
-7.18 \mathrm{a}\end{array}$ & $\begin{array}{r}-1.86 \\
-6.62 a\end{array}$ & $\begin{array}{r}-1.29 \\
-7.49 a\end{array}$ & $\begin{array}{r}-1.48 \\
-7.48 \mathrm{a}\end{array}$ & $\begin{array}{r}-2.11 \\
-7.49 a\end{array}$ & $\begin{array}{r}-2.06 \\
-7.42 \mathrm{a}\end{array}$ & $\begin{array}{r}-1.24 \\
-7.46 a\end{array}$ & $\begin{array}{r}-1.56 \\
-7.40 a\end{array}$ & $\begin{array}{r}-1.47 \\
-7.45 a\end{array}$ & $\begin{array}{r}-1.74 \\
-7.50 a\end{array}$ & $\begin{array}{r}-1.42 \\
-7.31 \mathrm{a}\end{array}$ \\
\hline Madagascar & $\begin{array}{l}\text { Level } \\
1^{\text {st }} \text { Diff }\end{array}$ & $\begin{array}{l}\text { na } \\
\text { na }\end{array}$ & $\begin{array}{r}-0.17 \\
-2.74 \mathrm{c}\end{array}$ & $\begin{array}{l}-1.63 \\
-1.83\end{array}$ & $\begin{array}{r}-0.99 \\
-5.33 a\end{array}$ & $\begin{array}{r}-0.88 \\
-5.39 a\end{array}$ & $\begin{array}{r}-0.58 \\
-5.71 \mathrm{a}\end{array}$ & $\begin{array}{l}-3.43 b \\
-6.74 a\end{array}$ & $\begin{array}{r}-2.44 \\
-7.62 \mathrm{a}\end{array}$ & $\begin{array}{r}-1.59 \\
-7.48 a\end{array}$ & $\begin{array}{r}-0.96 \\
-7.51 \mathrm{a}\end{array}$ & $\begin{array}{r}-0.61 \\
-7.51 \mathrm{a}\end{array}$ & $\begin{array}{r}-0.28 \\
-7.54 a\end{array}$ \\
\hline Malawi & $\begin{array}{l}\text { Level } \\
1^{\text {st }} \text { Diff }\end{array}$ & $\begin{array}{r}1.51 \\
-2.15\end{array}$ & $\begin{array}{r}0.8 \\
-8.30 a\end{array}$ & $\begin{array}{r}-1.43 \\
-6.23 a\end{array}$ & $\begin{array}{r}-1.06 \\
-6.13 a\end{array}$ & $\begin{array}{r}-1.15 \\
-6.11 a\end{array}$ & $\begin{array}{r}-1.17 \\
-5.60 a\end{array}$ & $\begin{array}{r}-0.97 \\
-5.11 a\end{array}$ & $\begin{array}{r}-1.69 \\
-8.74 a\end{array}$ & $\begin{array}{l}-2.89 c \\
-8.87 a\end{array}$ & $\begin{array}{r}-2.53 \\
-8.74 a\end{array}$ & $\begin{array}{r}-1.39 \\
-8.74 a\end{array}$ & $\begin{array}{r}-1.84 \\
-13.3 a\end{array}$ \\
\hline Mauritius & $\begin{array}{l}\text { Level } \\
1^{\text {st }} \text { Diff }\end{array}$ & $\begin{array}{l}-1.49 \\
-5.5 a\end{array}$ & $\begin{array}{r}-1.88 \\
-5.42 a\end{array}$ & $\begin{array}{r}-2.22 \\
-3.75 a\end{array}$ & $\begin{array}{l}-2.87 c \\
-4.45 a\end{array}$ & $\begin{array}{l}-3.16 b \\
-4.20 a\end{array}$ & $\begin{array}{r}-2.29 \\
-4.41 a\end{array}$ & $\begin{array}{r}-1.02 \\
-3.86 a\end{array}$ & $\begin{array}{l}\mathrm{s} \\
\mathrm{s}\end{array}$ & $\begin{array}{l}\mathrm{s} \\
\mathrm{s}\end{array}$ & $\begin{array}{l}\text { na } \\
\text { na }\end{array}$ & $\begin{array}{l}\text { na } \\
\text { na }\end{array}$ & $\begin{array}{l}\text { na } \\
\text { na }\end{array}$ \\
\hline Mozambique & $\begin{array}{l}\text { Level } \\
1^{\text {st }} \text { Diff }\end{array}$ & $\begin{array}{l}\text { na } \\
\text { na }\end{array}$ & $\begin{array}{l}\mathrm{s} \\
\mathrm{s}\end{array}$ & $\begin{array}{r}-0.47 \\
-4.74 a\end{array}$ & $\begin{array}{r}0.44 \\
-5.64 a\end{array}$ & $\begin{array}{r}0.12 \\
-6.39 a\end{array}$ & $\begin{array}{r}-1.39 \\
-6.06 a\end{array}$ & $\begin{array}{r}-7.25 a \\
-2.18\end{array}$ & $\begin{array}{l}-7.96 a \\
-3.92 a\end{array}$ & $\begin{array}{l}\mathrm{x} \\
\mathrm{x}\end{array}$ & $\begin{array}{l}\mathrm{x} \\
\mathrm{x}\end{array}$ & $\begin{array}{l}\mathrm{x} \\
\mathrm{x}\end{array}$ & $\begin{array}{l}\mathrm{x} \\
\mathrm{x}\end{array}$ \\
\hline Namibia & $\begin{array}{l}\text { Level } \\
1^{\text {st }} \text { Diff }\end{array}$ & $\begin{array}{r}-2.19 \\
-5.68 \mathrm{a}\end{array}$ & $\begin{array}{r}-2.26 \\
-6.31 \mathrm{a}\end{array}$ & $\begin{array}{l}-2.98 \mathrm{~b} \\
-7.13 \mathrm{a}\end{array}$ & $\begin{array}{r}-1.14 \\
-7.99 a\end{array}$ & $\begin{array}{l}-2.75 c \\
-3.43 b\end{array}$ & $\begin{array}{r}-2.01 \\
-2.99 b\end{array}$ & $\begin{array}{r}-1.47 \\
-2.83 c\end{array}$ & $\begin{array}{r}-1.24 \\
-3.09 b\end{array}$ & $\begin{array}{r}-1.79 \\
-2.55 \mathrm{se}\end{array}$ & $\begin{array}{l}-1.77 \\
-2.55\end{array}$ & $\begin{array}{r}-1.87 \\
-5.82 \mathrm{a}\end{array}$ & $\begin{array}{r}-1.15 \\
-3.61 \mathrm{a}\end{array}$ \\
\hline Seychelles & $\begin{array}{l}\text { Level } \\
1^{\text {st }} \text { Diff }\end{array}$ & $\begin{array}{r}0.44 \\
-2.05\end{array}$ & $\begin{array}{r}-1.16 \\
-6.97 a\end{array}$ & $\begin{array}{r}0.82 \\
-6.13 a\end{array}$ & $\begin{array}{r}-0.62 \\
-6.07 a\end{array}$ & $\begin{array}{r}0.59 \\
-7.57 \mathrm{a}\end{array}$ & $\begin{array}{r}-0.36 \\
-7.45 \mathrm{a}\end{array}$ & $\begin{array}{r}-1.07 \\
-7.44 a\end{array}$ & $\begin{array}{r}-1.45 \\
-7.67 a\end{array}$ & $\begin{array}{l}\mathrm{x} \\
\mathrm{x}\end{array}$ & $\begin{array}{l}\mathrm{x} \\
\mathrm{x}\end{array}$ & $\begin{array}{r}-0.19 \\
-7.94 a\end{array}$ & $\begin{array}{r}-0.89 \\
-8.01 \mathrm{a}\end{array}$ \\
\hline South Africa & $\begin{array}{l}\text { Level } \\
1^{\text {st }} \text { Diff }\end{array}$ & $\begin{array}{r}-0.84 \\
-7.99 a\end{array}$ & $\begin{array}{r}-1.94 \\
-7.62 \mathrm{a}\end{array}$ & $\begin{array}{r}-1.02 \\
-7.48 \mathrm{a}\end{array}$ & $\begin{array}{r}-0.56 \\
-8.16 a\end{array}$ & $\begin{array}{r}-1.73 \\
-4.71 \mathrm{a}\end{array}$ & $\begin{array}{r}-1.87 \\
-4.39 a\end{array}$ & $\begin{array}{r}-1.56 \\
-4.10 \mathrm{a}\end{array}$ & $\begin{array}{r}-1.56 \\
-4.31 \mathrm{a}\end{array}$ & $\begin{array}{r}-1.68 \\
-2.94 b\end{array}$ & $\begin{array}{l}-1.84 \\
-2.46\end{array}$ & $\begin{array}{l}-2.06 \\
-2.22\end{array}$ & $\begin{array}{r}-1.15 \\
-3.56 a\end{array}$ \\
\hline Swaziland & $\begin{array}{l}\text { Level } \\
1^{\text {st }} \text { Diff }\end{array}$ & $\begin{array}{l}-1.18 \\
-7.48\end{array}$ & $\begin{array}{r}-0.16 \\
-7.56 a\end{array}$ & $\begin{array}{r}0.53 \\
-7.70 a\end{array}$ & $\begin{array}{r}-0.57 \\
-8.06 a\end{array}$ & $\begin{array}{r}-1.14 \\
-7.95 a\end{array}$ & $\begin{array}{r}-1.80 \\
-6.09 a\end{array}$ & $\begin{array}{r}-0.05 \\
-5.89 a\end{array}$ & $\begin{array}{r}-0.26 \\
-6.06 a\end{array}$ & $\begin{array}{r}-1.56 \\
-2.59 c\end{array}$ & $\begin{array}{r}-2.3 \\
-2.28\end{array}$ & $\begin{array}{l}-2.09 \\
-2.16\end{array}$ & $\begin{array}{r}-1.67 \\
-3.28 b\end{array}$ \\
\hline Tanzania & $\begin{array}{l}\text { Level } \\
1^{\text {st }} \text { Diff }\end{array}$ & $\begin{array}{r}3.37 \mathrm{~b} \\
-2.24 \mathrm{~s}\end{array}$ & $\begin{array}{r}-1.12 \\
-5.41 \mathrm{a}\end{array}$ & $\begin{array}{r}-1.56 \\
-6.17 a\end{array}$ & $\begin{array}{l}-1.57 \\
-6.35 a\end{array}$ & $\begin{array}{r}-1.68 \\
-5.16 a\end{array}$ & $\begin{array}{l}-3.68 a \\
-6.95 a\end{array}$ & $\begin{array}{l}-3.74 a \\
-5.98 a\end{array}$ & $\begin{array}{r}-1.51 \\
-6.36 a\end{array}$ & $\begin{array}{r}-1.27 \\
-6.69 a\end{array}$ & $\begin{array}{r}-1.34 \\
-6.24 \mathrm{a}\end{array}$ & $\begin{array}{l}-3.33 b \\
-5.77 b\end{array}$ & $\begin{array}{r}1.42 \\
-7.01 \mathrm{a}\end{array}$ \\
\hline Zambia & $\begin{array}{l}\text { Level } \\
1^{\text {st }} \text { Diff }\end{array}$ & $\begin{array}{r}-1.63 \\
-3.48 b\end{array}$ & $\begin{array}{r}-1.93 \\
-4.03 a\end{array}$ & $\begin{array}{r}-2.13 \\
-4.66 a\end{array}$ & $\begin{array}{r}-0.83 \\
-5.45 a\end{array}$ & $\begin{array}{r}-2.29 \\
-5.51 \mathrm{a}\end{array}$ & $\begin{array}{r}-0.50 \\
-4.26 \mathrm{a}\end{array}$ & $\begin{array}{r}-0.84 \\
-5.03 a\end{array}$ & $\begin{array}{l}\mathrm{x} \\
\mathrm{x}\end{array}$ & $\begin{array}{r}-1 \\
-4.62 a\end{array}$ & $\begin{array}{r}-0.43 \\
-3.64 a\end{array}$ & $\begin{array}{r}-0.92 \\
-6.84 a\end{array}$ & $\begin{array}{r}-0.85 \\
-5.35 a\end{array}$ \\
\hline Zimbabwe & $\begin{array}{l}\text { Level } \\
1^{\text {st }} \text { Diff }\end{array}$ & $\begin{array}{r}-1.32 \\
-10.1 \mathrm{a} \\
\end{array}$ & $\begin{array}{l}-2.94 c \\
-10.3 a\end{array}$ & $\begin{array}{l}-4.09 a \\
-10.6 a\end{array}$ & $\begin{array}{r}-5.37 a \\
-2.44 \\
\end{array}$ & $\begin{array}{r}0.74 \\
-5.51 \\
\end{array}$ & $\begin{array}{r}5.42 \mathrm{a} \\
-3.91 \mathrm{a} \\
\end{array}$ & $\begin{array}{r}-0.95 \\
-5.06 a \\
\end{array}$ & $\begin{array}{r}-1.3 \\
-5.41 \mathrm{a} \\
\end{array}$ & $\begin{array}{r}-1.29 \\
-3.16 b \\
\end{array}$ & $\begin{array}{l}1.13 \\
0.28 \\
\end{array}$ & $\begin{array}{r}-2.02 \\
-7.32 \mathrm{a} \\
\end{array}$ & $\begin{array}{r}0.34 \\
-6.71 \mathrm{a} \\
\end{array}$ \\
\hline$\frac{\text { Panel B: Depc }}{\text { Angola }}$ & $\begin{array}{l}\text { osit rate } \\
\text { Level } \\
1^{\text {st }} \text { Diff }\end{array}$ & $\begin{array}{l}\text { na } \\
\text { na }\end{array}$ & $\begin{array}{l}\text { na } \\
\text { na }\end{array}$ & $\begin{array}{l}\text { na } \\
\text { na }\end{array}$ & $\begin{array}{l}\text { na } \\
\text { na }\end{array}$ & $\begin{array}{l}\text { na } \\
\text { na }\end{array}$ & $\begin{array}{r}-1.79 \\
-7.09 a\end{array}$ & $\begin{array}{l}-2.71 c \\
-10.7 a\end{array}$ & $\begin{array}{r}0 \\
-4.39 a\end{array}$ & $\begin{array}{r}-2.61 \mathrm{c} \\
12.4 \mathrm{a}\end{array}$ & $\begin{array}{r}-1.88 \\
-12.4 a\end{array}$ & $\begin{array}{r}-1.12 \\
-12.1 \mathrm{a}\end{array}$ & $\begin{array}{r} \\
-1.93 \\
-12.1 \mathrm{a}\end{array}$ \\
\hline Botswana & $\begin{array}{l}\text { Level } \\
1^{\text {st }} \text { Diff }\end{array}$ & $\begin{array}{r}-2.21 \\
-3.53 b\end{array}$ & $\begin{array}{r}-0.71 \\
-8.58 \mathrm{a}\end{array}$ & $\begin{array}{r}-0.67 \\
-8.71 \mathrm{a}\end{array}$ & $\begin{array}{r}-1.8 \\
-9.31 \mathrm{a}\end{array}$ & $\begin{array}{r}-1.39 \\
-12.7 \mathrm{a}\end{array}$ & $\begin{array}{r}-4.08 \mathrm{a} \\
-12.07 \mathrm{a}\end{array}$ & $\begin{array}{c}-0.87 \\
-6.26 a\end{array}$ & $\begin{array}{r}-1.08 \\
-6.78 a\end{array}$ & $\begin{array}{r}-0.29 \\
-7.81 \mathrm{a}\end{array}$ & $\begin{array}{r}-1.95 \\
-7.47 a\end{array}$ & $\begin{array}{r}-2.51 \\
-7.89 a\end{array}$ & $\begin{array}{r}-1.61 \\
-8.27 a\end{array}$ \\
\hline DRC & $\begin{array}{l}\text { Level } \\
1^{\text {st }} \text { Diff }\end{array}$ & $\begin{array}{l}\mathrm{s} \\
\mathrm{s}\end{array}$ & $\begin{array}{l}\mathrm{s} \\
\mathrm{s}\end{array}$ & $\begin{array}{l}\mathrm{x} \\
\mathrm{x}\end{array}$ & $\begin{array}{l}\mathrm{x} \\
\mathrm{x}\end{array}$ & $\begin{array}{l}\mathrm{x} \\
\mathrm{x}\end{array}$ & $\begin{array}{l}\mathrm{x} \\
\mathrm{x}\end{array}$ & $\begin{array}{l}\mathrm{x} \\
\mathrm{x}\end{array}$ & $\begin{array}{l}\mathrm{x} \\
\mathrm{x}\end{array}$ & $\begin{array}{l}\text { na } \\
\text { na }\end{array}$ & $\begin{array}{l}\text { na } \\
\text { na }\end{array}$ & $\begin{array}{l}\text { na } \\
\text { na }\end{array}$ & $\begin{array}{l}\text { na } \\
\text { na }\end{array}$ \\
\hline Lesotho & $\begin{array}{l}\text { Level } \\
1^{\text {st }} \text { Diff }\end{array}$ & $\begin{array}{r}-1.21 \\
-7.89 a\end{array}$ & $\begin{array}{r}-1.02 \\
-7.74 a\end{array}$ & $\begin{array}{l}-1.57 \\
-7.83 a\end{array}$ & $\begin{array}{r}-1.28 \\
-7.41 \mathrm{a}\end{array}$ & $\begin{array}{r}-2.2 \\
-7.95 \mathrm{a}\end{array}$ & $\begin{array}{r}-0.48 \\
-8.75 a\end{array}$ & $\begin{array}{r}-0.76 \\
-8.17 a\end{array}$ & $\begin{array}{r}-0.85 \\
-8.00 \mathrm{a}\end{array}$ & $\begin{array}{r}-1.66 \\
-8.19 a\end{array}$ & $\begin{array}{l}-5.22 a \\
-7.76 a\end{array}$ & $\begin{array}{r}-1.92 \\
-8.84 a\end{array}$ & $\begin{array}{r}-2.29 \\
-12.3 a\end{array}$ \\
\hline Madagascar & $\begin{array}{l}\text { Level } \\
1^{\text {st }} \text { Diff }\end{array}$ & $\begin{array}{r}-0.73 \\
-6.86 a\end{array}$ & $\begin{array}{r}-2.16 \\
-7.50 \mathrm{a}\end{array}$ & $\begin{array}{r}-1.5 \\
-6.41 \mathrm{a}\end{array}$ & $\begin{array}{r}-2.39 \\
-7.13 a\end{array}$ & $\begin{array}{r}-1.13 \\
-6.61 \mathrm{a}\end{array}$ & $\begin{array}{r}-1.48 \\
-5.91 \mathrm{a}\end{array}$ & $\begin{array}{r}-1.54 \\
-7.48 \mathrm{a}\end{array}$ & $\begin{array}{r}-1.42 \\
-7.50 \mathrm{a}\end{array}$ & $\begin{array}{r}-1.63 \\
-3.44 b\end{array}$ & $\begin{array}{r}-2.31 \\
-7.55 a\end{array}$ & $\begin{array}{r}-1.5 \\
-1.63 \mathrm{se}\end{array}$ & $\begin{array}{r}0.58 \\
-7.63 a\end{array}$ \\
\hline Malawi & $\begin{array}{l}\text { Level } \\
1^{\text {st }} \text { Diff }\end{array}$ & $\begin{array}{r}-0.66 \\
-11.3 a\end{array}$ & $\begin{array}{r}-0.52 \\
-7.72 \mathrm{a}\end{array}$ & $\begin{array}{r}-1.26 \\
-6.62 \mathrm{a}\end{array}$ & $\begin{array}{r}-0.57 \\
-6.58 \mathrm{a}\end{array}$ & $\begin{array}{r}-0.99 \\
-6.12 \mathrm{a}\end{array}$ & $\begin{array}{r}-0.92 \\
-6.85 a\end{array}$ & $\begin{array}{r}-0.95 \\
-5.75 a\end{array}$ & $\begin{array}{r}-1.34 \\
-7.42 \mathrm{a}\end{array}$ & $\begin{array}{l}-2.87 \mathrm{c} \\
-7.40 \mathrm{a}\end{array}$ & $\begin{array}{r}-1.11 \\
-7.50 \mathrm{a}\end{array}$ & $\begin{array}{r}-0.36 \\
-7.60 \mathrm{a}\end{array}$ & $\begin{array}{r}-1.16 \\
-7.85 a\end{array}$ \\
\hline Mauritius & $\begin{array}{l}\text { Level } \\
1^{\text {st }} \text { Diff }\end{array}$ & $\begin{array}{r}-1.45 \\
-7.68 \mathrm{a}\end{array}$ & $\begin{array}{r}-1.48 \\
-7.81 \mathrm{a}\end{array}$ & $\begin{array}{r}-1.84 \\
-8.27 \mathrm{a}\end{array}$ & $\begin{array}{r}-1.69 \\
-8.75 a\end{array}$ & $\begin{array}{r}-1.9 \\
-8.50 \mathrm{a}\end{array}$ & $\begin{array}{r}-1.95 \\
-9.03 a\end{array}$ & $\begin{array}{l}-2.86 c \\
-8.66 a\end{array}$ & $\begin{array}{l}-2.76 c \\
-8.45 a\end{array}$ & $\begin{array}{l}-3.01 b \\
-6.86 a\end{array}$ & $\begin{array}{l}-3.01 b \\
-8.78 a\end{array}$ & $\begin{array}{r}-1.87 \\
-8.19 a\end{array}$ & $\begin{array}{r}-1.93 \\
-8.13 a\end{array}$ \\
\hline Mozambique & $\begin{array}{l}\text { Level } \\
1^{\text {st }} \text { Diff }\end{array}$ & $\begin{array}{l}\mathrm{s} \\
\mathrm{s}\end{array}$ & $\begin{array}{l}\mathrm{s} \\
\mathrm{s}\end{array}$ & $\begin{array}{r}-1.46 \\
-5.77 a\end{array}$ & $\begin{array}{r}-1.6 \\
-6.79 a\end{array}$ & $\begin{array}{r}-1.37 \\
-7.67 \mathrm{a}\end{array}$ & $\begin{array}{r}-1.82 \\
-7.84 a\end{array}$ & $\begin{array}{r}-1.58 \\
-7.76 a\end{array}$ & $\begin{array}{r}2.57 \\
-3.55 b\end{array}$ & $\begin{array}{r}-0.56 \\
-7.28 \mathrm{a}\end{array}$ & $\begin{array}{r}-1.41 \\
-4.85 a\end{array}$ & $\begin{array}{r}-1.24 \\
-4.98 a\end{array}$ & $\begin{array}{r}-0.41 \\
-4.63 a\end{array}$ \\
\hline Namibia & $\begin{array}{l}\text { Level } \\
1^{\text {st }} \text { Diff }\end{array}$ & $\begin{array}{r}-1.47 \\
-6.44 a\end{array}$ & $\begin{array}{r}-1.65 \\
-7.98 a\end{array}$ & $\begin{array}{r}-0.69 \\
-7.59 \mathrm{a}\end{array}$ & $\begin{array}{r}-0.72 \\
-7.96 a\end{array}$ & $\begin{array}{r}-1.11 \\
-6.17 \mathrm{a}\end{array}$ & $\begin{array}{r}-1.64 \\
-4.61 \mathrm{a}\end{array}$ & $\begin{array}{r}-1 \\
-4.04 a\end{array}$ & $\begin{array}{r}-0.88 \\
-4.56 \mathrm{a}\end{array}$ & $\begin{array}{r}-1.14 \\
-3.12 b\end{array}$ & $\begin{array}{r}-2.23 \\
-2.64 \mathrm{c}\end{array}$ & $\begin{array}{l}-2.81 c \\
-3.39 b\end{array}$ & $\begin{array}{r}-0.66 \\
-3.56 \mathrm{a}\end{array}$ \\
\hline Seychelles & $\begin{array}{l}\text { Level } \\
1^{\text {st }} \text { Diff }\end{array}$ & $\begin{array}{r}-1.62 \\
-7.51 \mathrm{a}\end{array}$ & $\begin{array}{r}-1.55 \\
-7.50 \mathrm{a}\end{array}$ & $\begin{array}{r}-1.98 \\
-6.86 a\end{array}$ & $\begin{array}{r}-2.12 \\
-6.93 a\end{array}$ & $\begin{array}{r}0.46 \\
-6.15 a\end{array}$ & $\begin{array}{r}0.24 \\
-6.32 \mathrm{a}\end{array}$ & $\begin{array}{r}-0.51 \\
-6.58 \mathrm{a}\end{array}$ & $\begin{array}{r}-1.28 \\
-6.75 a\end{array}$ & $\begin{array}{l}-5.38 a \\
-5.07 a\end{array}$ & $\begin{array}{r}1.01 \\
-3.55 b\end{array}$ & $\begin{array}{r}-2.13 \\
-6.38 a\end{array}$ & $\begin{array}{r}-1.78 \\
-8.81 \mathrm{a}\end{array}$ \\
\hline South Africa & $\begin{array}{l}\text { Level } \\
1^{\text {st }} \text { Diff }\end{array}$ & $\begin{array}{r}-1.09 \\
-6.54 a\end{array}$ & $\begin{array}{r}-1.85 \\
-7.48 \mathrm{a}\end{array}$ & $\begin{array}{r}-1.39 \\
-7.42 \mathrm{a}\end{array}$ & $\begin{array}{r}-0.91 \\
-8.59 a\end{array}$ & $\begin{array}{r}-1.69 \\
-6.74 a\end{array}$ & $\begin{array}{r}-2.44 \\
-6.06 \mathrm{a}\end{array}$ & $\begin{array}{r}-1.7 \\
-5.64 a\end{array}$ & $\begin{array}{l}-1.73 \\
5.64 a\end{array}$ & $\begin{array}{r}-1.28 \\
-3.51 b\end{array}$ & $\begin{array}{r}-2.26 \\
-4.81 \mathrm{a}\end{array}$ & $\begin{array}{r}-0.95 \\
-4.80 a\end{array}$ & $\begin{array}{r}-1.19 \\
-4.62 \mathrm{a}\end{array}$ \\
\hline Swaziland & $\begin{array}{l}\text { Level } \\
1^{\text {st }} \text { Diff }\end{array}$ & $\begin{array}{r}-1.65 \\
-5.99 a\end{array}$ & $\begin{array}{r}-0.83 \\
-6.63 a\end{array}$ & $\begin{array}{r}-0.2 \\
-6.92 a\end{array}$ & $\begin{array}{r}-0.47 \\
-8.47 a\end{array}$ & $\begin{array}{r}-0.93 \\
-7.87 a\end{array}$ & $\begin{array}{r}-1.64 \\
-5.94 a\end{array}$ & $\begin{array}{r}-0.19 \\
-5.89 a\end{array}$ & $\begin{array}{r}-0.52 \\
-5.89 a\end{array}$ & $\begin{array}{r}-1.36 \\
-5.88 a\end{array}$ & $\begin{array}{r}-2.22 \\
-2.79 \mathrm{c}\end{array}$ & $\begin{array}{l}-2.49 \\
-1.19\end{array}$ & $\begin{array}{l}-1.88 \\
-1.92\end{array}$ \\
\hline Tanzania & $\begin{array}{l}\text { Level } \\
1^{\text {st }} \text { Diff }\end{array}$ & $\mathrm{S}$ & $\begin{array}{l}\mathrm{S} \\
\mathrm{S}\end{array}$ & $\begin{array}{r}-0.91 \\
-4.39 a\end{array}$ & $\begin{array}{r}-0.73 \\
-4.75 a\end{array}$ & $\begin{array}{r}-1.12 \\
-5.18 a\end{array}$ & $\begin{array}{r}-1.79 \\
-5.55 a\end{array}$ & $\begin{array}{l}-5.89 a \\
-4.85 a\end{array}$ & $\begin{array}{l}-1.60 \\
7.98 \mathrm{a}\end{array}$ & $\begin{array}{r}0.07 \\
-10.5 a\end{array}$ & $\begin{array}{r}-1.26 \\
-9.83 a\end{array}$ & $\begin{array}{r}-2.24 \\
-9.41 a\end{array}$ & $\begin{array}{r}-2.35 \\
-8.57 a\end{array}$ \\
\hline Zambia & $\begin{array}{l}\text { Level } \\
1^{\text {st }} \text { Diff }\end{array}$ & $\begin{array}{r}-1.56 \\
-3.86 a\end{array}$ & $\begin{array}{r}-1.92 \\
-3.83 a\end{array}$ & $\begin{array}{r}-1.91 \\
-3.88 a\end{array}$ & $\begin{array}{r}-2.12 \\
-4.21 \mathrm{a}\end{array}$ & $\begin{array}{l}-2.62 c \\
-4.25 a\end{array}$ & $\begin{array}{r}-1.05 \\
-5.90 a\end{array}$ & $\begin{array}{r}-0.98 \\
-6.43 a\end{array}$ & $\begin{array}{r}-3.38 b \\
-1.92\end{array}$ & $\begin{array}{r}-2.08 \\
-5.47 a\end{array}$ & $\begin{array}{r}-1.84 \\
-5.84 a\end{array}$ & $\begin{array}{r}-0.74 \\
-3.93 a\end{array}$ & $\begin{array}{r}-0.71 \\
-4.38 a\end{array}$ \\
\hline Zimbabwe & $\begin{array}{l}\text { Level } \\
1^{\text {st }} \text { Diff }\end{array}$ & $\begin{array}{r}-1.33 \\
-6.26 a \\
\end{array}$ & $\begin{array}{l}-2.60 c \\
-6.52 a \\
\end{array}$ & $\begin{array}{r}-0.88 \\
-6.96 a \\
\end{array}$ & $\begin{array}{r}-1.99 \\
-6.70 \mathrm{a}\end{array}$ & $\begin{array}{r}-0.58 \\
-6.93 a \\
\end{array}$ & $\begin{array}{r}2.34 \\
-5.58 \mathrm{a}\end{array}$ & $\begin{array}{r}-0.10 \\
-6.69 a \\
\end{array}$ & $\begin{array}{r}-1.51 \\
-4.71 \mathrm{a}\end{array}$ & $\begin{array}{r}-1.42 \\
-4.73 a \\
\end{array}$ & $\begin{array}{r}-1.9 \\
-0.86\end{array}$ & $\begin{array}{l}-3.57 \mathrm{a} \\
-11.2 \mathrm{a}\end{array}$ & $\begin{array}{r}-1.40 \\
-11.0 \mathrm{a}\end{array}$ \\
\hline Panel C: Lend & ding rate & & & & & & & & & & & & \\
\hline Angola & $\begin{array}{l}\text { Level } \\
1^{\text {st }} \text { Diff }\end{array}$ & $\begin{array}{l}\text { na } \\
\text { na }\end{array}$ & $\begin{array}{l}\text { na } \\
\text { na }\end{array}$ & $\begin{array}{l}\text { na } \\
\text { na }\end{array}$ & $\begin{array}{l}\text { na } \\
\text { na }\end{array}$ & $\begin{array}{l}\text { na } \\
\text { na }\end{array}$ & $\begin{array}{r}-2.34 \\
-6.49 a\end{array}$ & $\begin{array}{l}-3.47 \mathrm{~b} \\
-8.79 \mathrm{a}\end{array}$ & $\begin{array}{r}-2.22 \\
-7.44 a\end{array}$ & $\begin{array}{r}-1.71 \\
-6.79 a\end{array}$ & $\begin{array}{l}-3.40 b \\
-4.79 a\end{array}$ & $\begin{array}{l}-3.06 \mathrm{~b} \\
-7.81 \mathrm{a}\end{array}$ & $\begin{array}{r}-0.99 \\
-9.33 a\end{array}$ \\
\hline
\end{tabular}


Rolling period from January to December of the years

\begin{tabular}{llllllllllllll}
\cline { 3 - 7 } Country & Series & $1990-1994$ & $1991-1995$ & $1992-1996$ & $1993-1997$ & $1994-1998$ & $1995-1999$ & $1996-2000$ & $1997-2001$ & $1998-2002$ & $1999-2003$ & $2000-2004$ & $2001-2005$ \\
\hline
\end{tabular}

\begin{tabular}{|c|c|c|c|c|c|c|c|c|c|c|c|c|c|}
\hline Botswana & $\begin{array}{l}\text { Level } \\
1^{\text {st }} \text { Diff }\end{array}$ & $\begin{array}{r}-1.6 \\
-7.10 \mathrm{a}\end{array}$ & $\begin{array}{r}-2.45 \\
-9.27 a\end{array}$ & $\begin{array}{l}-4.05 a \\
-8.23 a\end{array}$ & $\begin{array}{r}-2.33 \\
-8.66 a\end{array}$ & $\begin{array}{r}-2.1 \\
-8.07 a\end{array}$ & $\begin{array}{r}-0.81 \\
-6.99 a\end{array}$ & $\begin{array}{r}0.08 \\
-6.77 a\end{array}$ & $\begin{array}{r}-0.36 \\
-6.77 a\end{array}$ & $\begin{array}{r}-1.09 \\
-6.79 a\end{array}$ & $\begin{array}{r}-1.52 \\
-6.53 a\end{array}$ & $\begin{array}{r}-1.95 \\
-5.65 a\end{array}$ & $\begin{array}{r}-2.23 \\
-5.94 a\end{array}$ \\
\hline DRC & $\begin{array}{l}\text { Level } \\
1^{\text {st }} \text { Diff }\end{array}$ & $\begin{array}{l}\text { na } \\
\text { na }\end{array}$ & $\begin{array}{l}\mathrm{s} \\
\mathrm{s}\end{array}$ & $\begin{array}{r}-0.83 \\
-5.70 a\end{array}$ & $\begin{array}{r}0.05 \\
-5.88 a\end{array}$ & $\begin{array}{r}-0.44 \\
-6.60 a\end{array}$ & $\begin{array}{r}-2.32 \\
-6.61 a\end{array}$ & $\begin{array}{r}-1.51 \\
-6.17 a\end{array}$ & $\begin{array}{r}-1.74 \\
-5.95 a\end{array}$ & $\begin{array}{r}-1.11 \\
-7.52 a\end{array}$ & $\begin{array}{r}-0.84 \\
-2.82 \mathrm{c}\end{array}$ & $\begin{array}{r}-0.21 \\
-6.40 \mathrm{a}\end{array}$ & $\begin{array}{l}\mathrm{s} \\
\mathrm{s}\end{array}$ \\
\hline Lesotho & $\begin{array}{l}\text { Level } \\
1^{\text {st }} \text { Diff }\end{array}$ & $\begin{array}{r}-0.48 \\
-6.82 a\end{array}$ & $\begin{array}{r}-1.88 \\
-13.1 \mathrm{a}\end{array}$ & $\begin{array}{l}-2.68 c \\
-12.6 a\end{array}$ & $\begin{array}{r}-2.27 \\
-8.61 \mathrm{a}\end{array}$ & $\begin{array}{r}-0.94 \\
-6.36 a\end{array}$ & $\begin{array}{r}-2.2 \\
-6.72 \mathrm{a}\end{array}$ & $\begin{array}{r}-2.04 \\
-6.63 a\end{array}$ & $\begin{array}{r}-1.58 \\
-6.53 a\end{array}$ & $\begin{array}{l}-3.84 a \\
-5.31 a\end{array}$ & $\begin{array}{r}-1.73 \\
-7.60 \mathrm{a}\end{array}$ & $\begin{array}{r}0.28 \\
-5.90 \mathrm{a}\end{array}$ & $\begin{array}{r}-1.08 \\
-5.79 a\end{array}$ \\
\hline Madagascar & $\begin{array}{l}\text { Level } \\
1^{\text {st }} \text { Diff }\end{array}$ & $\begin{array}{r}0.23 \\
-7.21 \mathrm{a}\end{array}$ & $\begin{array}{r}0.78 \\
-9.30 a\end{array}$ & $\begin{array}{r}-1.01 \\
-8.61 \mathrm{a}\end{array}$ & $\begin{array}{r}-1.45 \\
-8.42 \mathrm{a}\end{array}$ & $\begin{array}{r}-1.28 \\
-8.26 a\end{array}$ & $\begin{array}{r}-0.89 \\
-8.26 a\end{array}$ & $\begin{array}{r}-2.27 \\
-8.08 \mathrm{a}\end{array}$ & $\begin{array}{r}-1.74 \\
-7.82 \mathrm{a}\end{array}$ & $\begin{array}{r}-0.7 \\
-7.62 \mathrm{a}\end{array}$ & $\begin{array}{r}-0.61 \\
-7.74 a\end{array}$ & $\begin{array}{r}-1.81 \\
-7.56 a\end{array}$ & $\begin{array}{r}-0.61 \\
-7.52 a\end{array}$ \\
\hline Malawi & $\begin{array}{l}\text { Level } \\
1^{\text {st }} \text { Diff }\end{array}$ & $\begin{array}{r}-0.48 \\
-7.59 a\end{array}$ & $\begin{array}{r}-0.25 \\
-7.75 a\end{array}$ & $\begin{array}{r}-1.52 \\
-7.07 a\end{array}$ & $\begin{array}{r}-1.2 \\
-7.01 \mathrm{a}\end{array}$ & $\begin{array}{r}-1.29 \\
-6.74 a\end{array}$ & $\begin{array}{r}-1.33 \\
-6.84 a\end{array}$ & $\begin{array}{r}-0.55 \\
-5.50 a\end{array}$ & $\begin{array}{r}-1.07 \\
-7.59 \mathrm{a}\end{array}$ & $\begin{array}{l}-3.35 b \\
-7.60 a\end{array}$ & $\begin{array}{r}-1.44 \\
-7.16 a\end{array}$ & $\begin{array}{r}-0.41 \\
-7.59 a\end{array}$ & $\begin{array}{r}-1.05 \\
-7.96 a\end{array}$ \\
\hline Mauritius & $\begin{array}{l}\text { Level } \\
1^{\text {st }} \text { Diff }\end{array}$ & $\begin{array}{r}-1.89 \\
-3.76 a\end{array}$ & $\begin{array}{r}-0.65 \\
-5.23 a\end{array}$ & $\begin{array}{r}-0.9 \\
-6.16 a\end{array}$ & $\begin{array}{r}-2.24 \\
-6.40 \mathrm{a}\end{array}$ & $\begin{array}{l}-2.98 b \\
-6.32 a\end{array}$ & $\begin{array}{l}-3.04 b \\
-6.61 a\end{array}$ & $\begin{array}{l}-2.89 c \\
-6.54 a\end{array}$ & $\begin{array}{l}-2.92 b \\
-7.89 a\end{array}$ & $\begin{array}{l}-3.64 a \\
-9.89 a\end{array}$ & $\begin{array}{l}-5.13 a \\
-11.5 a\end{array}$ & $\begin{array}{l}-5.22 a \\
-6.08 a\end{array}$ & $\begin{array}{l}-8.26 a \\
-5.44 a\end{array}$ \\
\hline Mozambique & $\begin{array}{l}\text { Level } \\
1^{\text {st }} \text { Diff }\end{array}$ & $\begin{array}{l}\text { na } \\
\text { na }\end{array}$ & $\begin{array}{l}\text { na } \\
\text { na }\end{array}$ & $\begin{array}{l}\text { na } \\
\text { na }\end{array}$ & $\begin{array}{l}\mathrm{s} \\
\mathrm{s}\end{array}$ & $\begin{array}{l}\mathrm{s} \\
\mathrm{s}\end{array}$ & $\begin{array}{r}-2.52 \\
-5.57 a\end{array}$ & $\begin{array}{l}-3.03 b \\
-6.55 a\end{array}$ & $\begin{array}{l}-3.24 \mathrm{~b} \\
-7.22 \mathrm{a}\end{array}$ & $\begin{array}{r}-0.12 \\
-7.80 \mathrm{a}\end{array}$ & $\begin{array}{r}-1.13 \\
-6.16 a\end{array}$ & $\begin{array}{r}-1.26 \\
-6.19 a\end{array}$ & $\begin{array}{r}-0.84 \\
-4.64 a\end{array}$ \\
\hline Namibia & $\begin{array}{l}\text { Level } \\
1^{\text {st }} \text { Diff }\end{array}$ & $\begin{array}{r}-1.69 \\
-10.3 a\end{array}$ & $\begin{array}{l}-2.65 c \\
-10.7 a\end{array}$ & $\begin{array}{r}-2.22 \\
-11.4 a\end{array}$ & $\begin{array}{r}-1.82 \\
-10.22 \mathrm{a}\end{array}$ & $\begin{array}{r}-1.28 \\
-11.1 \mathrm{a}\end{array}$ & $\begin{array}{r}-2.74 c \\
-10.28 a\end{array}$ & $\begin{array}{r}-0.7 \\
-10.5 a\end{array}$ & $\begin{array}{r}-0.82 \\
-9.88 a\end{array}$ & $\begin{array}{r}-1.11 \\
-9.87 a\end{array}$ & $\begin{array}{l}-3.08 b \\
-10.0 a\end{array}$ & $\begin{array}{r}-0.46 \\
-10.9 a\end{array}$ & $\begin{array}{r}-0.96 \\
-10.9 a\end{array}$ \\
\hline Seychelles & $\begin{array}{l}\text { Level } \\
1^{\text {st }} \text { Diff }\end{array}$ & $\begin{array}{r}-1.76 \\
-7.49 a\end{array}$ & $\begin{array}{r}-1.53 \\
-7.51 \mathrm{a}\end{array}$ & $\begin{array}{r}-0.35 \\
-9.31 a\end{array}$ & $\begin{array}{r}-2.02 \\
-4.71 \mathrm{a}\end{array}$ & $\begin{array}{r}-0.64 \\
-7.82 a\end{array}$ & $\begin{array}{r}-1.02 \\
-8.03 a\end{array}$ & $\begin{array}{r}-0.73 \\
-8.01 \mathrm{a}\end{array}$ & $\begin{array}{r}-1.09 \\
-8.07 a\end{array}$ & $\begin{array}{r}-2.57 \\
-8.30 a\end{array}$ & $\begin{array}{r}-2.01 \\
-7.34 a\end{array}$ & $\begin{array}{r}0.07 \\
-6.74 a\end{array}$ & $\begin{array}{r}-0.79 \\
-6.20 a\end{array}$ \\
\hline South Africa & $\begin{array}{l}\text { Level } \\
1^{\text {st }} \text { Diff }\end{array}$ & $\begin{array}{r}-0.74 \\
-7.35 a\end{array}$ & $\begin{array}{r}-1.72 \\
-7.16 a\end{array}$ & $\begin{array}{r}-1.03 \\
-6.73 a\end{array}$ & $\begin{array}{r}-0.73 \\
-7.36 a\end{array}$ & $\begin{array}{r}-1.72 \\
-5.22 a\end{array}$ & $\begin{array}{r}-1.93 \\
-4.93 a\end{array}$ & $\begin{array}{r}-1.35 \\
-4.74 a\end{array}$ & $\begin{array}{r}-1.45 \\
-4.66 a\end{array}$ & $\begin{array}{r}-1.45 \\
-3.57 a\end{array}$ & $\begin{array}{l}-1.71 \\
-2.44\end{array}$ & $\begin{array}{r}-2.13 \\
-3.12 b\end{array}$ & $\begin{array}{r}-1.7 \\
-3.18 b\end{array}$ \\
\hline Swaziland & $\begin{array}{l}\text { Level } \\
1^{\text {st }} \text { Diff }\end{array}$ & $\begin{array}{r}-1.21 \\
-6.78 a\end{array}$ & $\begin{array}{r}-0.02 \\
-6.91 a\end{array}$ & $\begin{array}{r}0.63 \\
-7.17 \mathrm{a}\end{array}$ & $\begin{array}{r}-0.53 \\
-7.15 a\end{array}$ & $\begin{array}{r}-1.11 \\
-7.69 a\end{array}$ & $\begin{array}{r}-1.81 \\
-5.86 a\end{array}$ & $\begin{array}{r}-0.06 \\
-5.89 a\end{array}$ & $\begin{array}{r}-0.26 \\
-6.06 a\end{array}$ & $\begin{array}{r}-1.56 \\
-2.59 c\end{array}$ & $\begin{array}{l}-2.33 \\
-2.34\end{array}$ & $\begin{array}{l}-2.14 \\
-2.18\end{array}$ & $\begin{array}{r}-1.69 \\
-3.29 b\end{array}$ \\
\hline Tanzania & $\begin{array}{l}\text { Level } \\
1^{\text {st }} \text { Diff }\end{array}$ & $\begin{array}{r}-0.73 \\
-5.48 a\end{array}$ & $\begin{array}{r}-0.6 \\
-6.93 a\end{array}$ & $\begin{array}{r}-1.38 \\
-7.45 a\end{array}$ & $\begin{array}{r}-0.68 \\
-7.39 a\end{array}$ & $\begin{array}{r}-0.42 \\
-7.54 a\end{array}$ & $\begin{array}{r}-5.81 a \\
-1.98\end{array}$ & $\begin{array}{l}-4.15 a \\
-8.07 a\end{array}$ & $\begin{array}{l}-3.77 a \\
-7.91 a\end{array}$ & $\begin{array}{r}-0.59 \\
-7.69 a\end{array}$ & $\begin{array}{r}-0.21 \\
-7.71 \mathrm{a}\end{array}$ & $\begin{array}{r}-0.58 \\
-7.72 a\end{array}$ & $\begin{array}{l}-2.96 b \\
-7.10 a\end{array}$ \\
\hline Zambia & $\begin{array}{l}\text { Level } \\
1^{\text {st }} \text { Diff }\end{array}$ & $\begin{array}{r}-1.42 \\
-4.85 a\end{array}$ & $\begin{array}{r}-1.47 \\
-4.93 a\end{array}$ & $\begin{array}{r}-1.55 \\
-4.95 a\end{array}$ & $\begin{array}{r}-1.6 \\
-5.30 a\end{array}$ & $\begin{array}{l}-4.61 a \\
-3.15 b\end{array}$ & $\begin{array}{r}-1.72 \\
-2.69 \mathrm{c}\end{array}$ & $\begin{array}{r}-2.80 c \\
-2.14\end{array}$ & $\begin{array}{r}-2.51 \\
-5.87 a\end{array}$ & $\begin{array}{r}-1.72 \\
-6.54 a\end{array}$ & $\begin{array}{r}-1.95 \\
-7.73 a\end{array}$ & $\begin{array}{r}-0.26 \\
-6.12 a\end{array}$ & $\begin{array}{r}-0.21 \\
-8.30 a\end{array}$ \\
\hline Zimbabwe & $\begin{array}{l}\text { Level } \\
1^{\text {st }} \text { Diff }\end{array}$ & $\begin{array}{r}-0.89 \\
-8.36 a\end{array}$ & $\begin{array}{r}-1.86 \\
-9.63 a\end{array}$ & $\begin{array}{l}-2.65 c \\
-9.58 a\end{array}$ & $\begin{array}{l}-4.50 a \\
-12.9 a\end{array}$ & $\begin{array}{r}0.97 \\
-11.3 a\end{array}$ & $\begin{array}{r}-1.41 \\
-9.39 a\end{array}$ & $\begin{array}{r}0.5 \\
-5.92 a\end{array}$ & $\begin{array}{r}-1.43 \\
-4.85 a\end{array}$ & $\begin{array}{r}-1.58 \\
-5.18 a\end{array}$ & $\begin{array}{l}0.48 \\
2.89\end{array}$ & $\begin{array}{r}-0.26 \\
-6.12 a\end{array}$ & $\begin{array}{r}-0.58 \\
-6.52 a\end{array}$ \\
\hline
\end{tabular}

Note: "na" indicates that the interest rate is missing for the respective period. "s" indicates that there were insufficient observations for the test to be carried out. "x"

indicates that the interest rate series was constant for the period. Significance levels regarding the rejection of the null hypothesisare indicated with a, b, and c for $1 \%, 5 \%$, and $10 \%$, respectively. 
Rolling period from January to December of the years

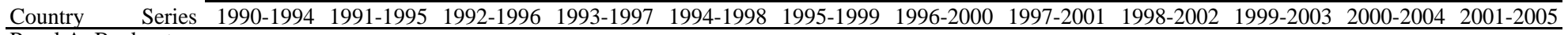
Panel A: Bank rate

\begin{tabular}{|c|c|c|c|c|c|c|c|c|c|c|c|c|c|}
\hline \multirow[t]{2}{*}{ Angola } & Level & n.a. & n.a. & n.a. & n.a. & n.a. & -1.7 & -1.38 & 0.01 & -0.27 & -0.59 & -1 & -0.44 \\
\hline & $1^{\text {st }}$ Diff & n.a. & n.a. & n.a. & n.a. & n.a. & $-7.55 a$ & $-7.55 a$ & $-7.85 a$ & $-7.75 a$ & $-7.72 \mathrm{a}$ & $-7.56 a$ & $-7.64 a$ \\
\hline \multirow[t]{2}{*}{ Botswana } & Level & -0.42 & -1.19 & -1.36 & -0.48 & -1.59 & -0.95 & -0.92 & -0.59 & 0.01 & -1.12 & $-2.27 b$ & -1.3 \\
\hline & $1^{\text {st }}$ Diff & $-7.79 a$ & $-7.40 \mathrm{a}$ & $-7.40 \mathrm{a}$ & $-7.46 a$ & -1.22 & $-5.72 \mathrm{a}$ & $-11.1 \mathrm{a}$ & $-11.3 a$ & $-8.91 \mathrm{a}$ & $-11.1 \mathrm{a}$ & $-11.3 a$ & $-6.10 \mathrm{a}$ \\
\hline \multirow[t]{2}{*}{ DRC } & Level & -0.44 & -0.44 & 0.28 & -1.13 & -1.02 & -1.27 & -1.05 & -0.84 & -0.97 & -0.84 & -0.3 & -0.52 \\
\hline & $1^{\text {st }}$ Diff & 0.14 & $-7.65 a$ & $-7.29 a$ & $-6.30 a$ & -6.32 & $-6.38 \mathrm{a}$ & $-5.88 a$ & 0.07 & $-7.50 \mathrm{a}$ & $-2.99 a$ & $-7.00 \mathrm{a}$ & $-6.10 \mathrm{a}$ \\
\hline \multirow[t]{2}{*}{ Lesotho } & Level & -0.47 & -1.19 & -1.02 & -1.29 & -1 & -1.15 & -1.76 & -1.31 & -1.51 & -0.84 & -0.71 & -1.12 \\
\hline & $1^{\text {st }}$ Diff & $-7.13 a$ & $-7.25 a$ & $-6.51 a$ & $-7.55 a$ & $-7.48 a$ & $-7.53 a$ & $-7.49 a$ & $-7.51 \mathrm{a}$ & 7.47a & $-7.49 a$ & $-7.53 a$ & $-7.36 a$ \\
\hline \multirow[t]{2}{*}{ Madagascar } & Level & C & -0.29 & -1.53 & -0.9 & -0.84 & -0.33 & -0.29 & -0.87 & -1.4 & -1.04 & -0.77 & -0.43 \\
\hline & $1^{\text {st }}$ Diff & C & $-2.66 \mathrm{a}$ & $-1.89 c$ & $-5.37 a$ & $-5.43 a$ & $-5.73 a$ & -1.3 & $-7.64 a$ & $-7.55 a$ & $-7.57 \mathrm{a}$ & $-7.58 \mathrm{a}$ & $-7.59 a$ \\
\hline \multirow[t]{2}{*}{ Malawi } & Level & 2.17 & 1.46 & -0.75 & -0.82 & -0.76 & -1.21 & -0.82 & -1.2 & -1.32 & $-2.58 b$ & -1.36 & -0.65 \\
\hline & $1^{\text {st }}$ Diff & $-2 . .27 b$ & $-8.10 \mathrm{a}$ & $-6.27 a$ & $-6.18 \mathrm{a}$ & $-6.16 a$ & $-5.65 a$ & $-5.12 \mathrm{a}$ & $-8.80 a$ & $-1.89 c$ & $-8.80 a$ & $-8.80 a$ & -0.6 \\
\hline \multirow[t]{2}{*}{ Mauritius } & Level & -1.42 & -1.65 & $-2.17 b$ & -1.74 & $-1.92 c$ & -0.78 & -1.08 & $\mathrm{~s}$ & $\mathrm{~s}$ & n.a. & n.a. & n.a. \\
\hline & $1^{\text {st }}$ Diff & $-5.49 a$ & $-5.46 a$ & $-3.79 a$ & $-4.48 a$ & $-4.24 a$ & $-3.04 a$ & $-3.86 a$ & $\mathrm{~s}$ & $\mathrm{~S}$ & n.a. & n.a. & n.a. \\
\hline Mozambique & Level & $\mathrm{s}$ & $\mathrm{s}$ & -0.63 & 0.13 & 0.06 & 0.15 & -0.88 & -0.72 & $\mathrm{x}$ & $\mathrm{x}$ & $\mathrm{x}$ & $\mathrm{x}$ \\
\hline & $1^{\text {st }}$ Diff & $\mathrm{s}$ & $\mathrm{s}$ & $-4.80 a$ & $-5.70 a$ & $-6.41 a$ & $-5.89 a$ & $-2.09 b$ & $-3.86 a$ & $\mathrm{x}$ & $\mathrm{x}$ & $\mathrm{x}$ & $\mathrm{x}$ \\
\hline Namibia & Level & -0.57 & -0.81 & -0.82 & -1.15 & $-1.88 c$ & $-1.99 b$ & -1.38 & -0.9 & -1.52 & 0.61 & -1.8 & -0.9 \\
\hline & $1^{\text {st }}$ Diff & $-5.65 a$ & $-6.35 a$ & -0.13 se & -0.75 & $-3.41 a$ & $-1.92 \mathrm{c}$ & $-2.84 a$ & $-3.07 a$ & $-2.57 b$ & -1.16 & $-5.84 a$ & $-3.62 a$ \\
\hline Seychelles & Level & -0.69 & -0.91 & 0.93 & -0.5 & 0.89 & 0.15 & -0.16 & -0.48 & $\mathrm{x}$ & $\mathrm{x}$ & -0.01 & -0.48 \\
\hline & $1^{\text {st }}$ Diff & -1.41 & $-7.73 a$ & $-6.07 a$ & $-6.04 a$ & $-6.85 a$ & $-7.30 \mathrm{a}$ & $-6.99 a$ & $-7.68 a$ & $\mathrm{x}$ & $\mathrm{x}$ & $-7.66 a$ & $-7.98 a$ \\
\hline South Africa & Level & 0.16 & -0.68 & -0.72 & -0.51 & -1 & -1.61 & -1.6 & -0.95 & -1.53 & -0.27 & $-2.05 b$ & -0.84 \\
\hline & $1^{\text {st }}$ Diff & $-7.89 a$ & -7.64 & $-7.55 a$ & -0.77 & $-4.69 a$ & $-3.35 a$ & $-4.14 a$ & $-4.32 \mathrm{a}$ & $-2.97 b$ & -0.96 & $-2.21 b$ & $-3.55 a$ \\
\hline Swaziland & Level & -1.19 & -0.4 & 0.34 & -0.16 & 0.22 & -1.08 & -0.54 & 0.43 & -1.32 & -0.51 & $-2.14 b$ & -1.6 \\
\hline & $1^{\text {st }}$ Diff & $-7.55 a$ & $-7.60 \mathrm{a}$ & $-7.70 a$ & -0.83 & $-7.87 a$ & $-6.15 a$ & $-5.91 a$ & $-6.03 a$ & $-2.62 b$ & $-2.31 b$ & $-2.16 b$ & $-3.29 a$ \\
\hline Tanzania & Level & 2.42 & -0.88 & -1.38 & -1.4 & -1.46 & -0.71 & -0.55 & -1.19 & -0.94 & -1.14 & -0.81 & -1.45 \\
\hline & $1^{\text {st }}$ Diff & $-2.41 b$ & $-5.45 a$ & $-6.23 a$ & $-6.40 a$ & 0.11 & $-6.95 a$ & $-4.08 a$ & $-4.03 a$ & $-4.86 a$ & $-2.24 b$ & $-5.44 a$ & -1.1 \\
\hline Zambia & Level & -1.58 & -1.84 & -2.01 & -0.75 & -1.06 & -0.61 & -0.76 & $\mathrm{X}$ & -0.94 & -0.50 & -0.74 & -0.61 \\
\hline & $1^{\text {st }}$ Diff & $-3.43 a$ & $-3.90 a$ & $-4.45 a$ & $-1.91 c$ & $-4.83 a$ & $-3.54 a$ & $-3.11 a$ & $\mathrm{x}$ & $-4.61 a$ & $-3.57 \mathrm{a}$ & $-6.68 a$ & $-2.68 \mathrm{a}$ \\
\hline Zimbabwe & Level & 0.11 & 0.14 & -1.2 & $-5.52 a$ & 0.57 & 0.95 & -0.73 & -0.67 & -0.96 & 0.18 & $-2.01 b$ & 0.39 \\
\hline & $1^{\text {st }}$ Diff & $-9.89 a$ & -1.36 & $-10.7 \mathrm{a}$ & $-2.04 b$ & $-5.50 a$ & $-3.82 \mathrm{a}$ & $-4.98 \mathrm{a}$ & $-5.42 a$ & -3.03 & -0.86 & $-7.38 \mathrm{a}$ & $-6.79 a$ \\
\hline Panel B: Dep & osit rate & & & & & & & & & & & & \\
\hline Angola & Level & n.a. & n.a. & n.a. & n.a. & n.a. & $-1.81 \mathrm{c}$ & -1.08 & 1.78 & $-2.29 b$ & $-1.91 c$ & -1.01 & -1.34 \\
\hline & $1^{\text {st }}$ Diff & n.a. & n.a. & n.a. & n.a. & n.a. & $-7.11 \mathrm{a}$ & $-1.63 c$ & $-4.45 a$ & $-12.5 \mathrm{a}$ & $-12.5 a$ & $-12.3 a$ & $-11.7 \mathrm{a}$ \\
\hline Botswana & Level & -0.72 & -0.79 & -0.73 & -0.49 & 0.3 & $-3.39 a$ & -0.96 & -1.1 & -0.29 & -1.12 & $-1.63 c$ & $-1.66 c$ \\
\hline & $1^{\text {st }}$ Diff & $-4.12 \mathrm{a}$ & $-3.60 \mathrm{a}$ & $-8.76 a$ & $-9.22 \mathrm{a}$ & -0.53 & $-11.67 a$ & $-6.31 a$ & $-6.84 a$ & -0.71 & $-7.52 a$ & $-7.67 a$ & $-6.57 a$ \\
\hline DRC & Level & $\mathrm{s}$ & $\mathrm{s}$ & $\mathrm{x}$ & $\mathrm{x}$ & $\mathrm{x}$ & $\mathrm{x}$ & $\mathrm{x}$ & $\mathrm{x}$ & n.a. & n.a. & n.a. & n.a. \\
\hline & $1^{\text {st }}$ Diff & $\mathrm{s}$ & $\mathrm{s}$ & $\mathrm{x}$ & $\mathrm{x}$ & $\mathrm{x}$ & $\mathrm{x}$ & $\mathrm{x}$ & $\mathrm{x}$ & n.a. & n.a. & n.a. & n.a. \\
\hline Lesotho & Level & -0.89 & -0.96 & -1.08 & -1.05 & -1.1 & -0.69 & 0.01 & -0.35 & -0.79 & -0.6 & -1.38 & $-2.21 b$ \\
\hline & $1^{\text {st }}$ Diff & $-7.95 a$ & -7.82 & $-7.89 a$ & $-7.47 \mathrm{a}$ & $-8.00 \mathrm{a}$ & -0.46 & $-8.14 a$ & $-7.57 \mathrm{a}$ & $-8.23 a$ & $-7.72 a$ & $-8.90 \mathrm{a}$ & $-12.4 \mathrm{a}$ \\
\hline Madagascar & Level & -0.5 & -2 & -1.3 & -2.01 & -0.87 & -1.48 & -1.5 & -1.23 & -1.23 & -0.92 & -1.41 & 0.14 \\
\hline & $1^{\text {st }}$ Diff & $-6.89 a$ & $-7.56 a$ & $-6.47 a$ & $-7.17 a$ & $-6.60 a$ & $-5.95 a$ & $-7.55 a$ & $-7.56 a$ & $-3.47 a$ & $-7.60 a$ & -1.68 & $-7.65 a$ \\
\hline Malawi & Level & -0.27 & 0.05 & -0.95 & -0.69 & -1.01 & -0.95 & -0.84 & -0.67 & -0.98 & -0.99 & -0.06 & -0.09 \\
\hline & $1^{\text {st }}$ Diff & -11.35 & $-7.70 \mathrm{a}$ & $-6.68 \mathrm{a}$ & $-6.64 a$ & $-6.17 a$ & -0.95 & $-5.76 a$ & $-5.90 a$ & $-3.01 \mathrm{a}$ & $-7.55 a$ & $-7.60 \mathrm{a}$ & $-7.81 \mathrm{a}$ \\
\hline Mauritius & Level & -1.27 & -1.35 & -1.58 & -1.3 & -1.64 & -1.41 & -1.22 & $-2.75 c$ & $-2.41 b$ & $-2.90 a$ & -1.59 & $-1.92 c$ \\
\hline & $1^{\text {st }}$ Diff & $-7.52 \mathrm{a}$ & $-7.88 \mathrm{a}$ & $-8.34 a$ & $-8.83 a$ & $-8.43 a$ & $-9.11 \mathrm{a}$ & $-8.74 a$ & $-8.52 a$ & $-7.41 \mathrm{a}$ & $-8.86 a$ & $-5.71 \mathrm{a}$ & $-8.12 \mathrm{a}$ \\
\hline Mozambique & Level & $\mathrm{s}$ & $\mathrm{s}$ & -1.39 & -1.58 & -1.42 & -0.82 & -1.59 & $-2.09 b$ & -0.38 & -1.07 & -0.85 & -0.46 \\
\hline & $1^{\text {st }}$ Diff & $\mathrm{s}$ & $\mathrm{s}$ & $-5.86 a$ & $-6.87 a$ & $-7.74 a$ & $-7.89 a$ & $-7.81 a$ & $-2.33 b$ & -1.23 & $-4.88 a$ & $-5.02 \mathrm{a}$ & $-4.30 \mathrm{a}$ \\
\hline Namibia & Level & -0.28 & -0.84 & -0.66 & -0.59 & -0.6 & -1.24 & -0.99 & -0.32 & -0.9 & -0.2 & $-2.67 a$ & -0.72 \\
\hline & $1^{\text {st }}$ Diff & $-6.52 a$ & $-8.05 a$ & $-7.64 a$ & $-8.02 \mathrm{a}$ & $-6.04 a$ & $-4.65 a$ & $-3.80 a$ & $-4.52 \mathrm{a}$ & $-3.18 a$ & -1.33 & $-2.66 a$ & $-3.47 a$ \\
\hline Seychelles & Level & -1.28 & -1.38 & -1.97 & $-1.84 c$ & 0.19 & 0.48 & -0.04 & -0.22 & -0.5 & 1.45 & $-2.12 b$ & -1.73 \\
\hline & $1^{\text {st }}$ Diff & -7.38 & $-7.55 a$ & $-6.89 a$ & $-6.30 a$ & $-5.76 a$ & $-6.37 a$ & $-6.56 a$ & -6.5 & $-6.28 a$ & $-2.59 b$ & $-1.66 c$ & $-8.65 a$ \\
\hline South Africa & Level & -0.65 & -0.61 & -0.82 & -0.68 & -0.7 & $-1.97 b$ & -1.75 & -1.37 & -1.65 & -0.12 & -1.05 & -1.05 \\
\hline & $1^{\text {st }}$ Diff & $-1.64 c$ & $-7.50 \mathrm{a}$ & $-2.21 b$ & $-7.73 a$ & $-7.75 a$ & $1.85 \mathrm{c}$ & $-5.66 a$ & $-5.67 a$ & $-3.30 a$ & $-3.28 a$ & $-4.73 a$ & $-4.61 \mathrm{a}$ \\
\hline Swaziland & Level & -1.66 & -0.82 & -0.44 & -0.4 & 0.73 & -1.16 & -0.69 & -0.25 & -0.99 & -0.3 & $-2.53 b$ & $-1.90 c$ \\
\hline & $1^{\text {st }}$ Diff & $-6.05 a$ & $-6.69 a$ & $-6.97 a$ & -0.79 & $-7.81 a$ & $-5.99 a$ & $-5.93 a$ & $-5.88 a$ & $-5.93 a$ & $-2.83 a$ & -1.92 & $-1.99 b$ \\
\hline Tanzania & Level & $\mathrm{S}$ & $\mathrm{S}$ & -1.15 & -0.85 & -0.88 & -0.6 & -0.26 & 0.34 & 0.29 & -0.74 & -1.06 & $-1.97 b$ \\
\hline & $1^{\text {st }}$ Diff & $\mathrm{s}$ & $\mathrm{s}$ & $-4.37 a$ & $-4.71 a$ & $-5.02 a$ & $-5.51 a$ & $-4.86 a$ & -0.76 & $-8.96 a$ & $-9.41 a$ & $-7.07 a$ & $-8.04 a$ \\
\hline Zambia & Level & -1.43 & -1.7 & $-1.89 c$ & $-1.84 c$ & -0.9 & -0.78 & -0.46 & -0.71 & -0.1 & -0.51 & -0.82 & -0.65 \\
\hline & $1^{\text {st }}$ Diff & $-3.89 a$ & $-3.86 a$ & $-2.69 a$ & $-2.17 b$ & $-4.00 a$ & -0.71 & $-6.33 a$ & -1.63 & $-4.47 a$ & -0.17 & $-3.93 a$ & $-2.69 a$ \\
\hline Zimbabwe & Level & -0.22 & -0.7 & -0.93 & -0.86 & -0.56 & 1.04 & 0.08 & -1.31 & -1.43 & -1.73 & $-3.24 a$ & -1.41 \\
\hline & $1^{\text {st }}$ Diff & $-6.16 a$ & $-5.40 \mathrm{a}$ & $-1.92 c$ & $-6.44 a$ & $-6.96 a$ & $-5.53 a$ & $-5.64 a$ & $-4.75 a$ & $-4.76 a$ & -0.71 & $-11.3 \mathrm{a}$ & $-10.5 a$ \\
\hline Panel C: Lenc & ding rate & & & & & & & & & & & & \\
\hline Angola & Level & n.a. & n.a. & n.a. & n.a. & n.a. & $-2.36 b$ & -1.59 & -0.64 & -0.95 & -1.09 & $-3.06 a$ & -1.11 \\
\hline & $1^{\text {st }}$ Diff & n.a. & n.a. & n.a. & n.a. & n.a. & $-6.55 a$ & $-8.86 a$ & $-7.50 a$ & $-6.82 a$ & $-4.83 a$ & $-7.72 \mathrm{a}$ & -0.94 \\
\hline Botswana & Level & -0.37 & -0.73 & -1.14 & $-2.31 b$ & -1.41 & -0.68 & -0.08 & 0.13 & 0.97 & -0.51 & -1.01 & $2.14 b$ \\
\hline & $1^{\text {st }}$ Diff & $-7.14 a$ & $-2.43 b$ & $-8.24 a$ & $-2.92 a$ & $-8.13 a$ & $-7.04 a$ & $-6.80 a$ & $-6.75 a$ & $-6.02 a$ & -1.57 & $-5.69 a$ & $-5.99 a$ \\
\hline DRC & Level & n.a. & $\mathrm{S}$ & -0.69 & 0.38 & 0.23 & -0.69 & -0.95 & -1.04 & -0.94 & -0.83 & -0.01 & n.a. \\
\hline & $1^{\text {st }}$ Diff & n.a. & $\mathrm{s}$ & $-5.74 a$ & $-5.88 a$ & $-6.58 a$ & $-6.62 a$ & $-6.00 a$ & $-6.00 a$ & $-2.15 b$ & $-2.85 a$ & $-6.43 a$ & n.a. \\
\hline Lesotho & Level & -1.13 & -1.22 & -1.48 & $-2.25 b$ & -0.21 & -1.46 & $-1.72 \mathrm{c}$ & -1.59 & $-4.01 a$ & 0.75 & -0.04 & -0.77 \\
\hline & $1^{\text {st }}$ Diff & $-6.52 a$ & $-13.2 \mathrm{a}$ & $-12.6 a$ & -1.35 & $-6.37 a$ & $-4.73 a$ & $-6.69 a$ & $-6.58 \mathrm{a}$ & $-4.92 a$ & $-7.58 \mathrm{a}$ & $-5.89 a$ & $-5.75 a$ \\
\hline Madagascar & Level & 0.11 & 0.92 & -0.49 & -0.85 & -0.94 & -0.87 & -0.03 & -0.16 & -0.43 & -0.06 & -0.81 & -0.81 \\
\hline
\end{tabular}


Rolling period from January to December of the years

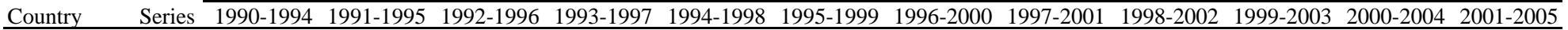

\begin{tabular}{|c|c|c|c|c|c|c|c|c|c|c|c|c|c|}
\hline & $1^{\text {st }}$ Diff & $-7.24 a$ & $-9.21 a$ & $-8.63 a$ & $-8.48 a$ & $-8.33 a$ & -1.09 & $-7.95 a$ & $-7.78 a$ & $-7.64 a$ & $-7.72 \mathrm{a}$ & $-7.60 \mathrm{a}$ & $-7.58 a$ \\
\hline \multirow[t]{2}{*}{ Malawi } & Level & -0.42 & 0.16 & -0.96 & -1.11 & -1.09 & -0.87 & -0.65 & -0.6 & -0.82 & -1.46 & -0.26 & -0.04 \\
\hline & $1^{\text {st }}$ Diff & $-7.63 a$ & $-7.73 a$ & $-7.12 \mathrm{a}$ & $-7.07 a$ & $-6.79 a$ & $-6.89 a$ & $-5.50 a$ & $-2.37 b$ & $-2.17 b$ & $-7.16 a$ & $-7.62 \mathrm{a}$ & $-7.92 \mathrm{a}$ \\
\hline \multirow[t]{2}{*}{ Mauritius } & Level & -1.92 & -0.73 & -0.82 & $-1.68 c$ & $-1.96 b$ & $-2.81 c$ & $-2.91 b$ & $-2.94 a$ & $-2.34 b$ & -1.2 & $-5.27 a$ & -0.94 \\
\hline & $1^{\text {st }}$ Diff & $-3.76 a$ & $-5.28 a$ & $-6.21 a$ & $-6.44 a$ & $-6.37 a$ & $-6.41 a$ & $-6.50 a$ & $-7.95 a$ & $-9.98 a$ & $-11.6 a$ & $-6.13 a$ & $-5.49 a$ \\
\hline \multirow[t]{2}{*}{ Mozambique } & Level & n.a. & n.a. & n.a. & $\mathrm{s}$ & $\mathrm{s}$ & -1.41 & -1.12 & -1.16 & -0.32 & -0.81 & -0.99 & -0.84 \\
\hline & $1^{\text {st }}$ Diff & n.a. & n.a. & n.a. & $\mathrm{s}$ & $\mathrm{s}$ & $-5.54 a$ & $-6.59 a$ & $-7.28 a$ & -1.22 & $-6.17 a$ & $-6.25 a$ & $-4.68 a$ \\
\hline \multirow[t]{2}{*}{ Namibia } & Level & -0.15 & -0.55 & -1.16 & $-1.71 c$ & -0.48 & -1.35 & -0.79 & -0.47 & -0.93 & 0 & 0.002 & -0.21 \\
\hline & $1^{\text {st }}$ Diff & $-8.97 a$ & $-9.72 \mathrm{a}$ & $-8.72 \mathrm{a}$ & $-10.3 a$ & $-10.7 a$ & $-8.67 a$ & $-10.5 a$ & -9.62 & $-9.81 a$ & $-9.89 a$ & $-1.91 c$ & $-10.9 a$ \\
\hline \multirow[t]{2}{*}{ Seychelles } & Level & -1.77 & -1.53 & -0.08 & $-2.03 b$ & -0.66 & 0.11 & -0.16 & -0.2 & -0.38 & 0.41 & 0.64 & -0.39 \\
\hline & $1^{\text {st }}$ Diff & $-7.16 a$ & $-7.30 \mathrm{a}$ & $-8.04 a$ & $-4.77 a$ & $-7.89 a$ & $-7.69 a$ & $-7.96 a$ & $-7.97 a$ & $-3.44 a$ & $-6.96 a$ & $-6.67 a$ & $-5.95 a$ \\
\hline \multirow[t]{2}{*}{ South Africa } & Level & 0.2 & -0.72 & -0.77 & -0.65 & -0.98 & -1.62 & -1.41 & -0.92 & -1.31 & -0.29 & $-2.18 b$ & $-1.63 c$ \\
\hline & $1^{\text {st }}$ Diff & $-7.28 \mathrm{a}$ & $-7.20 \mathrm{a}$ & $-6.79 a$ & -0.95 & $-5.20 \mathrm{a}$ & $-3.57 a$ & $-4.77 a$ & $-4.67 a$ & $-3.60 \mathrm{a}$ & -1.3 & $-3.13 a$ & $-3.18 a$ \\
\hline \multirow[t]{2}{*}{ Swaziland } & Level & -1.1 & -0.16 & 0.36 & -0.09 & 0.27 & -1.09 & -0.54 & 0.44 & -1.32 & -0.49 & $-2.18 b$ & -1.64 \\
\hline & $1^{\text {st }}$ Diff & $-6.84 a$ & $-6.43 a$ & $-6.68 a$ & $-7.15 a$ & $-7.61 \mathrm{a}$ & $-5.92 a$ & $-5.92 \mathrm{a}$ & $-6.02 a$ & $-2.62 \mathrm{a}$ & $-2.38 b$ & $-2.19 b$ & $-3.30 a$ \\
\hline \multirow[t]{2}{*}{ Tanzania } & Level & -0.62 & -0.28 & -1.24 & -0.81 & -0.06 & -0.11 & -0.25 & 0.24 & 0.37 & 0.34 & 0.05 & -0.52 \\
\hline & $1^{\text {st }}$ Diff & $-5.54 a$ & $-6.80 a$ & $-7.21 \mathrm{a}$ & $-7.45 a$ & $-7.54 \mathrm{a}$ & $-1.64 c$ & $-7.94 a$ & -1.09 & $-7.76 a$ & $-7.35 a$ & $-7.66 a$ & $.7 .16 \mathrm{a}$ \\
\hline \multirow[t]{2}{*}{ Zambia } & Level & -1.2 & -1.31 & -1.38 & -1.16 & -1.42 & -1.56 & $-2.01 b$ & -0.9 & -0.8 & -1.55 & -0.38 & -0.22 \\
\hline & $1^{\text {st }}$ Diff & $-4.89 a$ & $-4.97 a$ & -4.66 & -1.02 & $-2.26 b$ & $-2.51 b$ & -1.55 & $-2.63 b$ & $-6.40 a$ & $-7.74 a$ & $-5.79 a$ & -0.61 \\
\hline \multirow[t]{2}{*}{ Zimbabwe } & Level & -0.23 & -0.78 & -1.19 & $-2.30 b$ & 0.92 & 2.21 & 1.19 & -1.21 & -1.37 & -0.36 & -0.39 & -0.41 \\
\hline & $1^{\text {st }}$ Diff & $-8.35 a$ & $-8.08 a$ & $-8.09 a$ & $-12.4 a$ & $-11.4 a$ & $-9.33 a$ & $-5.56 a$ & $-4.81 a$ & $-5.21 a$ & -0.43 & $-5.96 a$ & $-6.48 a$ \\
\hline
\end{tabular}

See notes to Table B-5. 
Table B-7: Engle-Granger cointegration analysis

\begin{tabular}{|c|c|c|c|c|c|c|c|c|c|c|c|}
\hline \multirow[t]{2}{*}{ Country } & \multicolumn{2}{|c|}{ Rolling sample } & \multirow[t]{2}{*}{ Obs } & \multicolumn{2}{|c|}{ Intercept } & \multicolumn{2}{|c|}{ Slope } & \multirow[t]{2}{*}{ DW } & \multirow[t]{2}{*}{$\overline{\mathrm{DF}}$} & \multirow[t]{2}{*}{ ADF } & \multirow[t]{2}{*}{ Coint? } \\
\hline & From & To & & Coeff & t-stat & Coeff & t-stat & & & & \\
\hline \multicolumn{12}{|c|}{$\begin{array}{l}\text { Panel A: Cointegration of national lending rate and national central bank policy rate } \\
\end{array}$} \\
\hline \multirow[t]{7}{*}{ Angola } & Jan-95 & Dec-99 & 60 & -51.98 & -3.14 & 2.03 & 12.11 & 0.66 & -3.33 & & yes \\
\hline & Jan-96 & Dec-00 & 60 & -37.64 & -2.05 & 1.62 & 8.57 & 0.52 & -3.30 & -2.50 & yes \\
\hline & Jan-97 & Dec-01 & 60 & 13.50 & 3.68 & 0.63 & 17.88 & 0.61 & -3.32 & & yes \\
\hline & Jan-98 & Dec-02 & 60 & 19.01 & 3.81 & 0.56 & 13.87 & 0.55 & -3.04 & & yes \\
\hline & Jan-99 & Dec-03 & 60 & 34.34 & 3.89 & 0.45 & 6.95 & 0.63 & -3.42 & & yes \\
\hline & Jan-00 & Dec-04 & 60 & 58.17 & 7.79 & 0.27 & 4.99 & 1.04 & -4.41 & & yes \\
\hline & Jan-01 & Dec-05 & 60 & 30.51 & 5.57 & 0.44 & 10.66 & 0.83 & -3.42 & & yes \\
\hline \multirow[t]{12}{*}{ Botswana } & Jan-90 & Dec-94 & 60 & 0.92 & 2.18 & 0.95 & 28.04 & 0.47 & -2.88 & & yes \\
\hline & Jan-91 & Dec-95 & 60 & -1.55 & -1.56 & 1.14 & 15.53 & 0.73 & -3.81 & & yes \\
\hline & Jan-92 & Dec-96 & 60 & 7.25 & 6.49 & 0.52 & 6.37 & 0.44 & -3.28 & -2.76 & yes \\
\hline & Jan-93 & Dec-97 & 60 & 9.27 & 12.63 & 0.38 & 6.92 & 0.65 & -3.41 & & yes \\
\hline & Jan-94 & Dec-98 & 60 & 9.18 & 10.80 & 0.38 & 5.77 & 0.41 & -3.02 & & yes \\
\hline & Jan-95 & Dec-99 & 60 & 7.00 & 8.26 & 0.56 & 8.56 & 0.30 & -3.04 & & no \\
\hline & Jan-96 & Dec-00 & 60 & 2.42 & 3.76 & 0.93 & 18.76 & 1.81 & -6.93 & & yes \\
\hline & Jan-97 & Dec-01 & 60 & 2.15 & 4.11 & 0.95 & 24.10 & 1.87 & -7.15 & & yes \\
\hline & Jan-98 & Dec-02 & 60 & 1.98 & 4.04 & 0.97 & 26.96 & 1.89 & -7.34 & & yes \\
\hline & Jan-99 & Dec-03 & 60 & 3.36 & 5.70 & 0.87 & 20.96 & 1.73 & -7.73 & & yes \\
\hline & Jan-00 & Dec-04 & 60 & 5.04 & 7.64 & 0.76 & 16.49 & 1.75 & -6.82 & -1.88 & yes \\
\hline & Jan-01 & Dec-05 & 60 & 1.61 & 22.00 & 0.99 & 195.86 & 1.71 & -6.59 & & yes \\
\hline \multirow[t]{6}{*}{ DR Congo } & Jan-94 & Dec-98 & 60 & 67.28 & 2.70 & 1.29 & 7.23 & 0.09 & -1.77 & & no \\
\hline & Jan-95 & Dec-99 & 60 & 30.16 & 2.08 & 1.21 & 11.20 & 0.29 & -3.72 & & yes \\
\hline & Jan-96 & Dec-00 & 60 & 19.39 & 3.14 & 1.11 & 23.52 & 0.95 & -4.28 & & yes \\
\hline & Jan-97 & Dec-01 & 60 & 19.41 & 3.10 & 1.11 & 19.59 & 0.92 & -6.62 & -5.32 & yes \\
\hline & Jan-98 & Dec-02 & 60 & 12.16 & 3.55 & 1.20 & 33.80 & 1.31 & -5.45 & -3.08 & yes \\
\hline & Jan-99 & Dec-03 & 53 & 14.91 & 3.44 & 1.18 & 27.87 & 1.33 & -5.08 & & yes \\
\hline Lesotho & Jan-90 & Dec-94 & 60 & -1.13 & -0.57 & 1.19 & 9.50 & 1.03 & -4.50 & -3.16 & yes \\
\hline & Jan-91 & Dec-95 & 60 & -0.34 & -0.23 & 1.11 & 11.65 & 1.57 & -6.74 & & yes \\
\hline & Jan-92 & Dec-96 & 60 & 0.95 & 0.54 & 1.02 & 8.86 & 1.70 & -6.59 & & yes \\
\hline & Jan-93 & Dec-97 & 60 & -0.47 & -0.24 & 1.12 & 8.51 & 1.81 & -6.92 & & yes \\
\hline & Jan-94 & Dec-98 & 60 & -0.39 & -0.59 & 1.12 & 27.48 & 0.94 & -4.29 & & yes \\
\hline & Jan-95 & Dec-99 & 60 & 3.92 & 3.48 & 0.84 & 12.83 & 0.45 & -1.93 & & yes \\
\hline & Jan-96 & Dec-00 & 60 & 7.52 & 4.40 & 0.62 & 6.40 & 0.25 & -2.00 & & no \\
\hline & Jan-97 & Dec-01 & 60 & 8.15 & 6.22 & 0.58 & 7.73 & 0.23 & -1.87 & & no \\
\hline & Jan-98 & Dec-02 & 60 & 9.15 & 8.03 & 0.53 & 7.86 & 0.23 & -1.91 & & no \\
\hline & Jan-99 & Dec-03 & 60 & 11.37 & 9.64 & 0.35 & 4.97 & 0.23 & -1.76 & 0.44 & no \\
\hline & Jan-00 & Dec-04 & 60 & 7.95 & 5.29 & 0.52 & 5.29 & 0.09 & -0.70 & & no \\
\hline & Jan-01 & Dec-05 & 60 & -0.30 & -0.13 & 1.07 & 6.48 & 0.10 & -1.08 & & no \\
\hline Madagascar & Jan-91 & Dec-95 & 60 & 19.84 & 59.53 & 0.49 & 26.86 & 0.63 & -3.15 & -3.64 & yes \\
\hline & Jan-92 & Dec-96 & 60 & 19.65 & 37.82 & 0.53 & 21.57 & 0.39 & -1.91 & & yes \\
\hline & Jan-93 & Dec-97 & 60 & 22.63 & 34.62 & 0.43 & 13.91 & 0.26 & -1.99 & -1.39 & no \\
\hline & Jan-94 & Dec-98 & 60 & 24.02 & 48.42 & 0.38 & 16.11 & 0.40 & -2.82 & & yes \\
\hline & Jan-95 & Dec-99 & 60 & 23.86 & 51.61 & 0.39 & 17.18 & 0.36 & -3.50 & & yes \\
\hline & Jan-96 & Dec-00 & 60 & 22.45 & 44.34 & 0.47 & 14.95 & 0.25 & -1.90 & & no \\
\hline & Jan-97 & Dec-01 & 60 & 24.56 & 22.27 & 0.24 & 2.61 & 0.09 & -1.42 & & no \\
\hline & Jan-98 & Dec-02 & 60 & 24.33 & 45.59 & 0.19 & 3.91 & 0.12 & -1.52 & -1.11 & no \\
\hline & Jan-99 & Dec-03 & 60 & 21.99 & 68.15 & 0.36 & 12.25 & 0.40 & -3.18 & -2.81 & yes \\
\hline & Jan-00 & Dec-04 & 60 & 22.89 & 68.44 & 0.23 & 7.46 & 0.19 & -2.00 & & no \\
\hline & Jan-01 & Dec-05 & 60 & 23.58 & 100.36 & 0.14 & 6.74 & 0.30 & -1.69 & & no \\
\hline Malawi & Jan-90 & Dec-94 & 60 & 11.84 & 10.95 & 0.69 & 12.51 & 0.42 & -1.82 & -2.47 & yes \\
\hline & Jan-91 & Dec-95 & 60 & 8.84 & 7.52 & 0.84 & 19.90 & 0.53 & -2.96 & -4.07 & yes \\
\hline & Jan-92 & Dec-96 & 60 & 8.88 & 6.49 & 0.84 & 20.61 & 0.53 & -2.93 & -4.03 & yes \\
\hline & Jan-93 & Dec-97 & 60 & 9.03 & 5.86 & 0.83 & 18.66 & 0.50 & -2.90 & -4.01 & yes \\
\hline & Jan-94 & Dec-98 & 60 & 7.07 & 3.95 & 0.89 & 17.93 & 0.52 & -2.91 & -4.03 & yes \\
\hline & Jan-95 & Dec-99 & 60 & 3.92 & 2.01 & 1.00 & 20.41 & 0.43 & -4.09 & & yes \\
\hline & Jan-96 & Dec-00 & 60 & 3.06 & 2.56 & 1.05 & 34.99 & 0.34 & -2.73 & & no \\
\hline & Jan-97 & Dec-01 & 60 & 7.26 & 4.11 & 0.94 & 22.67 & 1.15 & -4.84 & & yes \\
\hline & Jan-98 & Dec-02 & 60 & 14.90 & 6.92 & 0.79 & 16.69 & 1.18 & -5.29 & & yes \\
\hline & Jan-99 & Dec-03 & 60 & 28.88 & 15.04 & 0.51 & 12.39 & 1.58 & -6.08 & & yes \\
\hline
\end{tabular}


Table B-7: Engle-Granger cointegration analysis

\begin{tabular}{|c|c|c|c|c|c|c|c|c|c|c|c|}
\hline \multirow[t]{2}{*}{ Country } & \multicolumn{2}{|c|}{ Rolling sample } & \multirow[t]{2}{*}{ Obs } & \multicolumn{2}{|c|}{ Intercept } & \multicolumn{2}{|c|}{ Slope } & \multirow[t]{2}{*}{$\overline{\mathrm{DW}}$} & \multirow[t]{2}{*}{$\mathrm{DF}$} & \multirow[t]{2}{*}{ ADF } & \multirow[t]{2}{*}{ Coint? } \\
\hline & From & To & & Coeff & t-stat & Coeff & t-stat & & & & \\
\hline \multirow[t]{2}{*}{ Malawi } & Jan-00 & Dec-04 & 60 & 17.96 & 11.56 & 0.72 & 20.55 & 1.41 & -5.63 & & yes \\
\hline & Jan-01 & Dec-05 & 60 & 15.48 & 13.11 & 0.76 & 26.21 & 1.36 & -5.61 & -2.72 & yes \\
\hline \multirow[t]{5}{*}{ Mauritius } & Jan-90 & Dec-94 & 60 & 11.98 & 25.36 & 0.54 & 12.27 & 0.27 & -0.92 & 0.51 & no \\
\hline & Jan-91 & Dec-95 & 60 & 10.55 & 13.21 & 0.73 & 9.79 & 0.18 & -0.95 & 0.23 & no \\
\hline & Jan-92 & Dec-96 & 60 & 9.12 & 11.89 & 0.93 & 12.88 & 0.34 & -2.63 & & no \\
\hline & Jan-93 & Dec-97 & 60 & 9.25 & 9.33 & 0.94 & 10.19 & 0.75 & -3.63 & -2.57 & yes \\
\hline & Jan-94 & Dec-98 & 55 & 12.62 & 9.84 & 0.65 & 5.73 & 0.75 & -3.44 & -2.50 & yes \\
\hline \multirow[t]{5}{*}{ Mozambique } & Jan-97 & Dec-01 & 53 & 9.09 & 5.05 & 1.22 & 7.42 & 0.63 & -2.91 & -1.60 & yes \\
\hline & Jan-98 & Dec-02 & 60 & 16.14 & 2.50 & 0.63 & 0.98 & 0.11 & -0.24 & & no \\
\hline & Jan-99 & Dec-03 & 60 & 22.56 & 54.47 & 0.00 & \#N/A & 0.06 & -1.15 & & no \\
\hline & Jan-00 & Dec-04 & 60 & 23.05 & 61.05 & 0.00 & $\# \mathrm{~N} / \mathrm{A}$ & 0.08 & -1.27 & & no \\
\hline & Jan-01 & Dec-05 & 60 & 23.13 & 63.94 & 0.00 & \#N/A & 0.08 & -0.86 & & no \\
\hline \multirow[t]{10}{*}{ Namibia } & Jan-92 & Dec-96 & 60 & 5.89 & 4.91 & 0.77 & 10.61 & 1.52 & -6.08 & & yes \\
\hline & Jan-93 & Dec-97 & 60 & 5.06 & 3.59 & 0.82 & 9.61 & 1.07 & -4.56 & & yes \\
\hline & Jan-94 & Dec-98 & 60 & 4.87 & 3.89 & 0.84 & 11.42 & 1.06 & -4.40 & & yes \\
\hline & Jan-95 & Dec-99 & 60 & 9.85 & 9.11 & 0.57 & 8.91 & 0.89 & -4.32 & -2.93 & yes \\
\hline & Jan-96 & Dec-00 & 60 & 8.01 & 12.31 & 0.68 & 16.84 & 1.10 & -4.83 & & yes \\
\hline & Jan-97 & Dec-01 & 60 & 6.83 & 16.20 & 0.77 & 26.86 & 1.19 & -4.95 & & yes \\
\hline & Jan-98 & Dec-02 & 60 & 5.65 & 9.48 & 0.83 & 18.89 & 0.67 & -3.37 & -2.09 & yes \\
\hline & Jan-99 & Dec-03 & 60 & 5.01 & 5.95 & 0.89 & 12.48 & 0.67 & -3.37 & -2.21 & yes \\
\hline & Jan-00 & Dec-04 & 60 & 6.05 & 9.17 & 0.76 & 12.13 & 0.78 & -3.79 & -2.32 & yes \\
\hline & Jan-01 & Dec-05 & 60 & 5.36 & 10.61 & 0.80 & 15.53 & 0.82 & -4.04 & -2.65 & yes \\
\hline South Africa & Jan-90 & Dec-94 & 60 & 3.92 & 16.19 & 0.96 & 60.50 & 1.79 & -6.89 & -6.77 & yes \\
\hline & Jan-91 & Dec-95 & 60 & 3.62 & 11.75 & 0.98 & 46.35 & 1.98 & -7.62 & & yes \\
\hline & Jan-92 & Dec-96 & 60 & 2.66 & 6.82 & 1.05 & 38.54 & 1.81 & -7.04 & & yes \\
\hline & Jan-93 & Dec-97 & 60 & 3.12 & 10.21 & 1.02 & 48.55 & 1.26 & -5.22 & & yes \\
\hline & Jan-94 & Dec-98 & 60 & 3.57 & 8.17 & 0.98 & 35.70 & 1.10 & -4.67 & & yes \\
\hline & Jan-95 & Dec-99 & 60 & 4.09 & 8.03 & 0.95 & 30.40 & 1.26 & -5.17 & & yes \\
\hline & Jan-96 & Dec-00 & 60 & 2.65 & 6.11 & 1.04 & 37.74 & 1.03 & -4.47 & & yes \\
\hline & Jan-97 & Dec-01 & 60 & 2.61 & 7.90 & 1.03 & 46.50 & 1.05 & -4.49 & & yes \\
\hline & Jan-98 & Dec-02 & 60 & 2.98 & 8.83 & 1.01 & 42.01 & 0.92 & -4.14 & & yes \\
\hline & Jan-99 & Dec-03 & 60 & 2.94 & 8.26 & 1.02 & 35.41 & 0.48 & -2.76 & -1.32 & yes \\
\hline & Jan-00 & Dec-04 & 60 & 3.94 & 13.53 & 0.93 & 35.30 & 0.11 & -1.46 & & no \\
\hline & Jan-01 & Dec-05 & 60 & 3.77 & 21.04 & 0.96 & 54.63 & 0.17 & -2.66 & & no \\
\hline Seychelles & Jan-90 & Dec-94 & 60 & 17.51 & 27.66 & -0.14 & -2.95 & 0.26 & -1.76 & & no \\
\hline & Jan-91 & Dec-95 & 60 & 17.90 & 39.65 & -0.17 & -4.94 & 0.31 & -2.20 & & no \\
\hline & Jan-92 & Dec-96 & 60 & 19.64 & 48.66 & -0.30 & -9.55 & 0.56 & -3.03 & & yes \\
\hline & Jan-93 & Dec-97 & 60 & 13.28 & 17.68 & 0.19 & 3.17 & 0.33 & -2.32 & & no \\
\hline & Jan-94 & Dec-98 & 60 & 11.15 & 30.07 & 0.37 & 11.61 & 0.41 & -2.53 & & yes \\
\hline & Jan-95 & Dec-99 & 60 & 9.49 & 35.61 & 0.52 & 20.12 & 0.30 & -1.97 & & no \\
\hline & Jan-96 & Dec-00 & 60 & 8.55 & 35.33 & 0.61 & 22.87 & 0.29 & -2.15 & & no \\
\hline & Jan-97 & Dec-01 & 60 & 8.18 & 35.68 & 0.64 & 21.14 & 0.15 & -1.27 & & no \\
\hline & Jan-98 & Dec-02 & 60 & 7.22 & 25.79 & 0.78 & 17.67 & 0.18 & -1.70 & & no \\
\hline & Jan-99 & Dec-03 & 60 & 8.69 & 6.78 & 0.49 & 2.08 & 0.15 & -2.72 & -2.44 & no \\
\hline & Jan-00 & Dec-04 & 60 & 8.37 & 45.11 & 0.52 & 14.24 & 0.59 & -3.16 & -3.86 & yes \\
\hline & Jan-01 & Dec-05 & 60 & 8.16 & 56.95 & 0.54 & 17.96 & 0.66 & -3.35 & -4.28 & yes \\
\hline Swaziland & Jan-90 & Dec-94 & 60 & 2.66 & 4.98 & 1.02 & 23.10 & 0.27 & -2.42 & -2.04 & no \\
\hline & Jan-91 & Dec-95 & 60 & 3.16 & 14.30 & 0.99 & 56.27 & 0.85 & -5.07 & & yes \\
\hline & Jan-92 & Dec-96 & 60 & 3.15 & 28.72 & 0.99 & 118.64 & 1.55 & -6.47 & & yes \\
\hline & Jan-93 & Dec-97 & 60 & 3.11 & 36.02 & 0.99 & 160.31 & 1.71 & -6.59 & & yes \\
\hline & Jan-94 & Dec-98 & 60 & 2.97 & 72.93 & 1.00 & 367.15 & 2.06 & -7.84 & & yes \\
\hline & Jan-95 & Dec-99 & 60 & 2.94 & 53.55 & 1.00 & 283.88 & 2.11 & -8.07 & -5.83 & yes \\
\hline & Jan-96 & Dec-00 & 60 & 3.00 & $7.42 \mathrm{E}+13$ & 1.00 & $3.71 \mathrm{E}+14$ & 0.00 & -1.04 & -0.85 & no \\
\hline & Jan-97 & Dec-01 & 60 & 3.00 & $1.49 \mathrm{E}+14$ & 1.00 & $6.99 \mathrm{E}+14$ & 0.00 & 1.42 & & no \\
\hline & Jan-98 & Dec-02 & 60 & 3.00 & $1.87 \mathrm{E}+14$ & 1.00 & $8.21 \mathrm{E}+14$ & 0.00 & -0.58 & -0.64 & no \\
\hline & Jan-99 & Dec-03 & 60 & 3.32 & 38.01 & 0.98 & 134.50 & 0.30 & -1.11 & & no \\
\hline & Jan-00 & Dec-04 & 60 & 4.13 & 42.30 & 0.91 & 99.32 & 0.26 & -2.03 & -1.40 & no \\
\hline & Jan-01 & Dec-05 & 60 & 4.16 & 58.70 & 0.91 & 128.37 & 0.29 & -2.26 & -1.65 & no \\
\hline Tanzania & Jan-92 & Dec-96 & 56 & 28.11 & 30.33 & 0.28 & 9.52 & 0.52 & -2.79 & -3.13 & yes \\
\hline
\end{tabular}


Table B-7: Engle-Granger cointegration analysis

\begin{tabular}{|c|c|c|c|c|c|c|c|c|c|c|c|}
\hline \multirow[t]{2}{*}{ Country } & \multicolumn{2}{|c|}{ Rolling sample } & \multirow[t]{2}{*}{ Obs } & \multicolumn{2}{|c|}{ Intercept } & \multicolumn{2}{|c|}{ Slope } & \multirow[t]{2}{*}{ DW } & \multirow[t]{2}{*}{ DF } & \multirow[t]{2}{*}{ ADF } & \multirow[t]{2}{*}{ Coint? } \\
\hline & From & To & & Coeff & t-stat & Coeff & t-stat & & & & \\
\hline \multirow[t]{9}{*}{ Tanzania } & Jan-93 & Dec-97 & 60 & 25.52 & 22.91 & 0.33 & 9.28 & 0.38 & -2.19 & & no \\
\hline & Jan-94 & Dec-98 & 60 & 21.04 & 14.08 & 0.43 & 8.97 & 0.27 & -2.63 & -2.33 & no \\
\hline & Jan-95 & Dec-99 & 60 & 17.07 & 13.55 & 0.51 & 11.32 & 0.31 & -2.81 & & no \\
\hline & Jan-96 & Dec-00 & 60 & 13.69 & 6.41 & 0.66 & 5.63 & 0.16 & -1.66 & -2.35 & no \\
\hline & Jan-97 & Dec-01 & 60 & 20.67 & 16.05 & 0.13 & 1.53 & 0.09 & -3.69 & -2.08 & yes \\
\hline & Jan-98 & Dec-02 & 60 & 15.74 & 18.89 & 0.36 & 6.12 & 0.14 & -1.16 & -2.14 & no \\
\hline & Jan-99 & Dec-03 & 60 & 14.17 & 11.30 & 0.39 & 3.96 & 0.07 & -0.31 & & no \\
\hline & Jan-00 & Dec-04 & 60 & 15.74 & 9.40 & 0.13 & 0.98 & 0.05 & -0.29 & & no \\
\hline & Jan-01 & Dec-05 & 60 & 21.03 & 16.74 & -0.42 & -4.07 & 0.12 & -2.85 & & no \\
\hline \multirow[t]{10}{*}{ Zambia } & Jan-92 & Dec-96 & 60 & 7.24 & 3.68 & 1.06 & 33.71 & 0.84 & -3.96 & -4.18 & yes \\
\hline & Jan-93 & Dec-97 & 60 & 14.23 & 7.08 & 0.97 & 29.29 & 0.52 & -2.98 & & yes \\
\hline & Jan-94 & Dec-98 & 52 & 19.08 & 8.55 & 0.83 & 16.30 & 0.34 & -2.66 & & no \\
\hline & Jan-95 & Dec-99 & 41 & 27.02 & 11.97 & 0.57 & 9.20 & 0.25 & -0.77 & -1.47 & no \\
\hline & Jan-96 & Dec-00 & 41 & 24.14 & 11.30 & 0.65 & 10.22 & 0.25 & -0.94 & -1.34 & no \\
\hline & Jan-97 & Dec-01 & 41 & 25.07 & 9.87 & 0.59 & 7.15 & 0.23 & -1.07 & -2.07 & no \\
\hline & Jan-98 & Dec-02 & 41 & 21.22 & 11.18 & 0.68 & 11.29 & 0.44 & -1.47 & -1.85 & yes \\
\hline & Jan-99 & Dec-03 & 49 & 27.46 & 14.27 & 0.50 & 8.07 & 0.53 & -2.49 & & yes \\
\hline & Jan-00 & Dec-04 & 60 & 24.88 & 25.05 & 0.57 & 16.44 & 0.48 & -2.71 & & yes \\
\hline & Jan-01 & Dec-05 & 60 & 21.68 & 20.96 & 0.68 & 17.35 & 0.38 & -2.43 & -2.44 & no \\
\hline \multirow[t]{12}{*}{ Zimbabwe } & Jan-90 & Dec-94 & 60 & 1.37 & 0.70 & 1.02 & 12.21 & 0.28 & -1.98 & & no \\
\hline & Jan-91 & Dec-95 & 60 & -2.19 & -0.67 & 1.18 & 9.51 & 0.38 & -2.45 & & no \\
\hline & Jan-92 & Dec-96 & 60 & -9.31 & -0.92 & 1.44 & 4.11 & 0.40 & -2.56 & & yes \\
\hline & Jan-93 & Dec-97 & 60 & 15.78 & 3.78 & 0.66 & 4.51 & 1.39 & -5.66 & & yes \\
\hline & Jan-94 & Dec-98 & 60 & -0.94 & -0.32 & 1.25 & 12.55 & 1.16 & -4.87 & & yes \\
\hline & Jan-95 & Dec-99 & 60 & 13.64 & 12.14 & 0.76 & 24.55 & 0.64 & -3.16 & -2.19 & yes \\
\hline & Jan-96 & Dec-00 & 60 & 13.77 & 11.16 & 0.78 & 28.68 & 0.24 & -1.10 & & no \\
\hline & Jan-97 & Dec-01 & 60 & 17.88 & 4.89 & 0.61 & 8.50 & 0.12 & -0.22 & -1.09 & no \\
\hline & Jan-98 & Dec-02 & 60 & 22.28 & 3.49 & 0.48 & 4.15 & 0.10 & -1.12 & -1.46 & no \\
\hline & Jan-99 & Dec-03 & 60 & -13.80 & -2.67 & 1.18 & 15.95 & 0.39 & -2.54 & & yes \\
\hline & Jan-00 & Dec-04 & 60 & -9.76 & -1.32 & 1.20 & 20.29 & 1.29 & -5.10 & & yes \\
\hline & Jan-01 & Dec-05 & 60 & 17.17 & 1.79 & 0.93 & 16.96 & 0.61 & -2.89 & -0.97 & yes \\
\hline \multicolumn{12}{|c|}{ Panel B: Cointegration of national lending rate and South African central bank policy rate } \\
\hline Botswana & Jan-90 & Dec-94 & 60 & 26.12 & 20.13 & -0.90 & -10.61 & 0.20 & -2.02 & -1.67 & no \\
\hline & Jan-91 & Dec-95 & 60 & 20.25 & 24.18 & -0.45 & -7.75 & 0.29 & -2.29 & & no \\
\hline & Jan-92 & Dec-96 & 60 & 15.10 & 24.10 & -0.05 & -1.21 & 0.36 & -3.79 & & yes \\
\hline & Jan-93 & Dec-97 & 60 & 14.85 & 33.97 & -0.04 & -1.17 & 0.43 & -2.42 & & yes \\
\hline & Jan-94 & Dec-98 & 60 & 14.21 & 42.47 & -0.01 & -0.38 & 0.22 & -2.15 & & no \\
\hline & Jan-95 & Dec-99 & 60 & 15.77 & 40.13 & -0.09 & -3.88 & 0.19 & -1.80 & -1.94 & no \\
\hline & Jan-96 & Dec-00 & 60 & 17.20 & 48.54 & -0.18 & -7.83 & 0.18 & -1.39 & -2.87 & no \\
\hline & Jan-97 & Dec-01 & 60 & 17.72 & 59.63 & -0.21 & -10.35 & 0.17 & -1.63 & -3.25 & yes \\
\hline & Jan-98 & Dec-02 & 60 & 17.94 & 47.08 & -0.20 & -7.46 & 0.11 & -1.13 & -3.24 & yes \\
\hline & Jan-99 & Dec-03 & 60 & 16.66 & 32.29 & -0.08 & -1.86 & 0.07 & -1.52 & -1.43 & no \\
\hline & Jan-00 & Dec-04 & 60 & 14.73 & 51.55 & 0.11 & 4.16 & 0.13 & -2.06 & & no \\
\hline & Jan-01 & Dec-05 & 60 & 14.77 & 92.89 & 0.12 & 7.65 & 0.26 & -2.14 & -2.57 & no \\
\hline Lesotho & Jan-90 & Dec-94 & 60 & 2.29 & 2.19 & 1.02 & 14.92 & 2.06 & -7.90 & & yes \\
\hline & Jan-91 & Dec-95 & 60 & 1.72 & 1.52 & 1.05 & 13.61 & 1.84 & -7.08 & & yes \\
\hline & Jan-92 & Dec-96 & 60 & 3.64 & 2.65 & 0.90 & 9.42 & 1.81 & -6.99 & & yes \\
\hline & Jan-93 & Dec-97 & 60 & 5.71 & 5.09 & 0.74 & 9.63 & 2.06 & -7.85 & & yes \\
\hline & Jan-94 & Dec-98 & 60 & 3.18 & 5.29 & 0.90 & 23.76 & 1.29 & -5.12 & & yes \\
\hline & Jan-95 & Dec-99 & 60 & 7.59 & 6.23 & 0.66 & 8.82 & 0.46 & -2.72 & -2.08 & yes \\
\hline & Jan-96 & Dec-00 & 60 & 10.97 & 11.86 & 0.48 & 8.16 & 0.47 & -2.97 & -2.58 & yes \\
\hline & Jan-97 & Dec-01 & 60 & 11.38 & 17.23 & 0.47 & 10.54 & 0.48 & -2.84 & & yes \\
\hline & Jan-98 & Dec-02 & 60 & 10.72 & 20.14 & 0.53 & 14.02 & 0.70 & -4.03 & & yes \\
\hline & Jan-99 & Dec-03 & 60 & 8.61 & 17.24 & 0.70 & 17.36 & 0.82 & -3.39 & & yes \\
\hline & Jan-00 & Dec-04 & 60 & 5.56 & 11.21 & 0.95 & 21.05 & 0.41 & -2.55 & & yes \\
\hline & Jan-01 & Dec-05 & 60 & 5.02 & 13.36 & 0.98 & 26.62 & 0.40 & -2.51 & -2.72 & yes \\
\hline Mozambique & Jan-97 & Dec-01 & 53 & 16.70 & 8.09 & 0.38 & 2.73 & 0.26 & -3.02 & & no \\
\hline & Jan-98 & Dec-02 & 60 & 20.88 & 11.60 & 0.12 & 0.92 & 0.10 & -0.13 & & no \\
\hline & Jan-99 & Dec-03 & 60 & 26.45 & 10.30 & -0.32 & -1.54 & 0.07 & -1.01 & & no \\
\hline
\end{tabular}


Table B-7: Engle-Granger cointegration analysis

\begin{tabular}{|c|c|c|c|c|c|c|c|c|c|c|c|}
\hline \multirow[t]{2}{*}{ Country } & \multicolumn{2}{|c|}{ Rolling sample } & \multirow[t]{2}{*}{ Obs } & \multicolumn{2}{|c|}{ Intercept } & \multicolumn{2}{|c|}{ Slope } & \multirow[t]{2}{*}{ DW } & \multirow[t]{2}{*}{ DF } & \multirow[t]{2}{*}{ ADF } & \multirow[t]{2}{*}{ Coint? } \\
\hline & From & To & & Coeff & t-stat & Coeff & t-stat & & & & \\
\hline \multirow[t]{2}{*}{ Mozambique } & Jan-00 & Dec-04 & 60 & 19.53 & 9.41 & 0.32 & 1.72 & 0.08 & -1.49 & & no \\
\hline & Jan-01 & Dec-05 & 60 & 14.68 & 13.61 & 0.85 & 8.05 & 0.20 & -2.53 & -3.75 & yes \\
\hline \multirow[t]{11}{*}{ Namibia } & Jan-91 & Dec-95 & 60 & 2.75 & 2.86 & 1.15 & 17.45 & 0.71 & -3.59 & -2.46 & yes \\
\hline & Jan-92 & Dec-96 & 60 & 9.63 & 9.27 & 0.63 & 8.68 & 1.27 & -5.22 & -2.62 & yes \\
\hline & Jan-93 & Dec-97 & 60 & 10.08 & 13.36 & 0.59 & 11.36 & 1.46 & -6.01 & & yes \\
\hline & Jan-94 & Dec-98 & 60 & 10.34 & 15.75 & 0.56 & 13.56 & 1.44 & -5.65 & & yes \\
\hline & Jan-95 & Dec-99 & 60 & 10.14 & 12.83 & 0.58 & 11.85 & 1.46 & -5.86 & -5.99 & yes \\
\hline & Jan-96 & Dec-00 & 60 & 7.36 & 10.34 & 0.73 & 16.25 & 1.19 & -4.94 & & yes \\
\hline & Jan-97 & Dec-01 & 60 & 6.37 & 12.23 & 0.79 & 22.59 & 0.96 & -4.32 & & yes \\
\hline & Jan-98 & Dec-02 & 60 & 5.48 & 8.25 & 0.81 & 17.18 & 0.64 & -3.31 & -2.31 & yes \\
\hline & Jan-99 & Dec-03 & 60 & 5.10 & 5.63 & 0.84 & 11.49 & 0.66 & -3.36 & -2.14 & yes \\
\hline & Jan-00 & Dec-04 & 60 & 6.49 & 10.31 & 0.69 & 12.05 & 0.84 & -4.05 & -2.41 & yes \\
\hline & Jan-01 & Dec-05 & 60 & 5.89 & 12.72 & 0.72 & 15.84 & 0.92 & -4.31 & -2.70 & yes \\
\hline \multirow[t]{12}{*}{ Swaziland } & Jan-90 & Dec-94 & 60 & 12.22 & 19.15 & 0.18 & 4.42 & 0.11 & -1.63 & -2.00 & no \\
\hline & Jan-91 & Dec-95 & 60 & 9.28 & 9.82 & 0.43 & 6.66 & 0.14 & -0.97 & -1.00 & no \\
\hline & Jan-92 & Dec-96 & 60 & 2.18 & 1.92 & 0.98 & 12.28 & 0.25 & -2.90 & -2.29 & no \\
\hline & Jan-93 & Dec-97 & 60 & -1.02 & -2.07 & 1.23 & 36.15 & 1.15 & -5.51 & -3.40 & yes \\
\hline & Jan-94 & Dec-98 & 60 & 5.71 & 6.94 & 0.77 & 14.86 & 0.30 & -2.28 & & no \\
\hline & Jan-95 & Dec-99 & 60 & 8.17 & 9.97 & 0.64 & 12.63 & 0.39 & -2.78 & -3.08 & yes \\
\hline & Jan-96 & Dec-00 & 60 & 5.64 & 7.83 & 0.78 & 17.17 & 0.29 & -2.06 & -2.49 & no \\
\hline & Jan-97 & Dec-01 & 60 & 4.72 & 8.30 & 0.83 & 21.70 & 0.30 & -2.30 & -2.82 & no \\
\hline & Jan-98 & Dec-02 & 60 & 5.47 & 11.24 & 0.76 & 21.98 & 0.34 & -2.46 & -3.60 & yes \\
\hline & Jan-99 & Dec-03 & 60 & 2.80 & 6.77 & 0.99 & 29.60 & 0.47 & -2.70 & -1.63 & yes \\
\hline & Jan-00 & Dec-04 & 60 & 4.62 & 13.96 & 0.84 & 27.87 & 0.08 & -1.32 & & no \\
\hline & Jan-01 & Dec-05 & 60 & 4.44 & 21.18 & 0.86 & 42.03 & 0.12 & -2.30 & & no \\
\hline \multirow[t]{12}{*}{ Zambia } & Jan-90 & Dec-94 & 60 & 212.42 & 11.86 & -9.83 & -8.41 & 0.15 & -0.64 & -2.48 & no \\
\hline & Jan-91 & Dec-95 & 60 & 201.96 & 8.43 & -9.44 & -5.74 & 0.13 & -1.37 & -2.61 & no \\
\hline & Jan-92 & Dec-96 & 60 & 206.36 & 7.27 & -9.78 & -4.92 & 0.13 & -1.32 & -2.58 & no \\
\hline & Jan-93 & Dec-97 & 60 & 199.19 & 7.82 & -9.21 & -5.27 & 0.10 & -1.25 & -2.73 & no \\
\hline & Jan-94 & Dec-98 & 60 & 111.17 & 9.93 & -3.93 & -5.56 & 0.10 & -2.27 & -3.85 & yes \\
\hline & Jan-95 & Dec-99 & 60 & 58.35 & 7.57 & -0.91 & -1.92 & 0.06 & -1.19 & -1.68 & no \\
\hline & Jan-96 & Dec-00 & 60 & 44.36 & 7.24 & -0.13 & -0.34 & 0.04 & -0.82 & -2.74 & no \\
\hline & Jan-97 & Dec-01 & 60 & 50.46 & 14.29 & -0.66 & -2.80 & 0.12 & -3.21 & & no \\
\hline & Jan-98 & Dec-02 & 60 & 57.41 & 25.68 & -1.24 & -7.77 & 0.23 & -2.25 & -2.72 & no \\
\hline & Jan-99 & Dec-03 & 60 & 47.50 & 16.69 & -0.43 & -1.86 & 0.26 & -1.70 & & no \\
\hline & Jan-00 & Dec-04 & 60 & 19.91 & 5.91 & 1.88 & 6.16 & 0.14 & -1.34 & -1.77 & no \\
\hline & Jan-01 & Dec-05 & 60 & 12.25 & 4.76 & 2.62 & 10.36 & 0.18 & -1.70 & -2.03 & no \\
\hline Panel C: Co & ion of $n$ & nal depos & e and & ational ce & al bank & y rate & & & & & \\
\hline Angola & Jan-95 & Dec-99 & 60 & -27.49 & -2.36 & 1.23 & 10.41 & 0.19 & -1.29 & & no \\
\hline & Jan-96 & Dec-00 & 60 & -16.33 & -1.17 & 0.90 & 6.25 & 0.13 & -1.88 & & no \\
\hline & Jan-97 & Dec-01 & 60 & 26.15 & 11.46 & 0.13 & 5.80 & 1.03 & -5.64 & -5.11 & yes \\
\hline & Jan-98 & Dec-02 & 60 & 30.27 & 9.95 & 0.10 & 4.06 & 1.16 & -4.84 & & yes \\
\hline & Jan-99 & Dec-03 & 60 & 33.02 & 4.37 & 0.05 & 0.91 & 0.66 & -3.28 & -1.91 & yes \\
\hline & Jan-00 & Dec-04 & 60 & -19.74 & -1.88 & 0.40 & 5.32 & 0.60 & -3.36 & -2.25 & yes \\
\hline & Jan-01 & Dec-05 & 60 & -30.62 & -4.07 & 0.47 & 8.28 & 0.62 & -3.34 & -2.06 & yes \\
\hline Botswana & Jan-90 & Dec-94 & 60 & -0.63 & -1.02 & 0.94 & 18.80 & 0.83 & -3.90 & -1.97 & yes \\
\hline & Jan-91 & Dec-95 & 60 & 3.61 & 1.36 & 0.59 & 2.99 & 0.40 & -2.44 & -1.46 & yes \\
\hline & Jan-92 & Dec-96 & 60 & -10.98 & -3.11 & 1.63 & 6.29 & 0.58 & -3.60 & -1.90 & yes \\
\hline & Jan-93 & Dec-97 & 60 & -10.16 & -3.82 & 1.55 & 7.82 & 0.73 & -3.58 & -2.48 & yes \\
\hline & Jan-94 & Dec-98 & 60 & 0.93 & 0.44 & 0.67 & 4.10 & 1.14 & -6.02 & -2.25 & yes \\
\hline & Jan-95 & Dec-99 & 60 & 3.29 & 2.03 & 0.46 & 3.71 & 1.07 & -4.58 & -5.83 & yes \\
\hline & Jan-96 & Dec-00 & 60 & 5.72 & 5.01 & 0.27 & 3.08 & 0.18 & -1.54 & & no \\
\hline & Jan-97 & Dec-01 & 60 & 3.50 & 3.85 & 0.44 & 6.42 & 0.26 & -2.23 & & no \\
\hline & Jan-98 & Dec-02 & 60 & -0.32 & -0.46 & 0.72 & 14.20 & 0.74 & -4.45 & & yes \\
\hline & Jan-99 & Dec-03 & 60 & 1.32 & 1.34 & 0.60 & 8.67 & 0.63 & -3.37 & & yes \\
\hline & Jan-00 & Dec-04 & 60 & 3.10 & 2.36 & 0.48 & 5.23 & 0.62 & -3.43 & & yes \\
\hline & Jan-01 & Dec-05 & 60 & 1.51 & 0.75 & 0.58 & 4.16 & 0.34 & -1.95 & & no \\
\hline Lesotho & Jan-90 & Dec-94 & 60 & -6.15 & -3.31 & 1.06 & 9.09 & 0.23 & -1.56 & & no \\
\hline & Jan-91 & Dec-95 & 60 & -3.67 & -1.54 & 0.92 & 6.07 & 0.17 & -1.44 & & no \\
\hline
\end{tabular}


Table B-7: Engle-Granger cointegration analysis

\begin{tabular}{|c|c|c|c|c|c|c|c|c|c|c|c|}
\hline \multirow[t]{2}{*}{ Country } & \multicolumn{2}{|c|}{ Rolling sample } & \multirow[t]{2}{*}{ Obs } & \multicolumn{2}{|c|}{ Intercept } & \multicolumn{2}{|c|}{ Slope } & \multirow[t]{2}{*}{ DW } & \multirow[t]{2}{*}{ DF } & \multirow[t]{2}{*}{ ADF } & \multirow[t]{2}{*}{ Coint? } \\
\hline & From & To & & Coeff & t-stat & Coeff & t-stat & & & & \\
\hline \multirow[t]{10}{*}{ Lesotho } & Jan-92 & Dec-96 & 60 & -3.89 & -1.22 & 0.96 & 4.56 & 0.16 & -1.51 & & no \\
\hline & Jan-93 & Dec-97 & 60 & -8.56 & -2.58 & 1.28 & 5.86 & 0.19 & -1.83 & & no \\
\hline & Jan-94 & Dec-98 & 60 & 6.94 & 3.57 & 0.28 & 2.32 & 0.19 & -2.09 & -2.47 & no \\
\hline & Jan-95 & Dec-99 & 60 & 22.25 & 10.06 & -0.65 & -5.02 & 0.30 & -1.91 & & no \\
\hline & Jan-96 & Dec-00 & 60 & 21.65 & 6.29 & -0.69 & -3.54 & 0.14 & -0.64 & & no \\
\hline & Jan-97 & Dec-01 & 60 & 2.94 & 1.02 & 0.29 & 1.76 & 0.07 & -1.18 & & no \\
\hline & Jan-98 & Dec-02 & 60 & -0.79 & -0.45 & 0.44 & 4.28 & 0.16 & -2.30 & & no \\
\hline & Jan-99 & Dec-03 & 60 & 0.67 & 0.66 & 0.29 & 4.79 & 0.34 & -5.08 & & yes \\
\hline & Jan-00 & Dec-04 & 60 & 3.06 & 7.53 & 0.12 & 4.50 & 0.35 & -2.25 & & no \\
\hline & Jan-01 & Dec-05 & 60 & 0.25 & 0.36 & 0.31 & 6.33 & 1.33 & -5.37 & & yes \\
\hline \multirow[t]{11}{*}{ Madagascar } & Jan-91 & Dec-95 & 60 & 21.52 & 72.47 & -0.11 & -7.03 & 0.62 & -3.25 & & yes \\
\hline & Jan-92 & Dec-96 & 60 & 20.96 & 37.61 & -0.08 & -3.03 & 0.81 & -3.83 & -3.08 & yes \\
\hline & Jan-93 & Dec-97 & 60 & 17.41 & 20.37 & 0.04 & 0.99 & 0.40 & -2.49 & -1.34 & yes \\
\hline & Jan-94 & Dec-98 & 60 & 9.95 & 9.05 & 0.31 & 5.92 & 0.24 & -2.08 & -1.47 & no \\
\hline & Jan-95 & Dec-99 & 60 & 6.85 & 8.23 & 0.39 & 9.76 & 0.37 & -2.27 & -1.53 & no \\
\hline & Jan-96 & Dec-00 & 60 & 6.25 & 6.76 & 0.49 & 8.68 & 0.34 & -3.03 & -1.33 & yes \\
\hline & Jan-97 & Dec-01 & 60 & 2.33 & 1.48 & 0.85 & 6.48 & 0.22 & -1.89 & & no \\
\hline & Jan-98 & Dec-02 & 60 & 3.58 & 2.41 & 0.75 & 5.69 & 0.12 & -1.32 & & no \\
\hline & Jan-99 & Dec-03 & 60 & 8.65 & 9.17 & 0.37 & 4.30 & 0.12 & -2.62 & -3.21 & yes \\
\hline & Jan-00 & Dec-04 & 60 & 8.31 & 23.20 & 0.44 & 13.45 & 0.64 & -3.90 & & yes \\
\hline & Jan-01 & Dec-05 & 60 & 7.58 & 16.86 & 0.52 & 13.14 & 0.43 & -1.86 & & yes \\
\hline \multirow[t]{12}{*}{ Malawi } & Jan-90 & Dec-94 & 60 & 2.06 & 2.22 & 0.83 & 17.58 & 0.99 & -3.37 & & yes \\
\hline & Jan-91 & Dec-95 & 60 & 4.34 & 5.72 & 0.72 & 26.59 & 0.60 & -3.03 & -3.87 & yes \\
\hline & Jan-92 & Dec-96 & 60 & 6.93 & 4.72 & 0.59 & 13.53 & 0.29 & -1.43 & & no \\
\hline & Jan-93 & Dec-97 & 60 & 0.94 & 0.37 & 0.71 & 9.56 & 0.14 & -1.29 & & no \\
\hline & Jan-94 & Dec-98 & 60 & -6.45 & -2.37 & 0.87 & 11.49 & 0.19 & -2.06 & & no \\
\hline & Jan-95 & Dec-99 & 60 & -15.86 & -8.92 & 1.06 & 23.87 & 0.52 & -2.96 & & yes \\
\hline & Jan-96 & Dec-00 & 60 & -14.61 & -13.55 & 1.01 & 37.35 & 0.87 & -4.01 & & yes \\
\hline & Jan-97 & Dec-01 & 60 & -8.50 & -4.91 & 0.84 & 20.82 & 1.06 & -4.61 & & yes \\
\hline & Jan-98 & Dec-02 & 60 & -2.37 & -1.09 & 0.72 & 15.05 & 1.11 & -4.87 & & yes \\
\hline & Jan-99 & Dec-03 & 60 & 2.95 & 1.00 & 0.60 & 9.58 & 0.95 & -4.17 & -3.42 & yes \\
\hline & Jan-00 & Dec-04 & 60 & -7.29 & -3.83 & 0.80 & 18.46 & 1.15 & -4.93 & & yes \\
\hline & Jan-01 & Dec-05 & 60 & -8.10 & -6.84 & 0.79 & 27.01 & 1.50 & -6.01 & -5.74 & yes \\
\hline \multirow[t]{5}{*}{ Mauritius } & Jan-90 & Dec-94 & 60 & 1.14 & 2.11 & 0.92 & 18.38 & 0.77 & -3.59 & & yes \\
\hline & Jan-91 & Dec-95 & 60 & 1.36 & 2.34 & 0.90 & 16.53 & 0.77 & -3.60 & & yes \\
\hline & Jan-92 & Dec-96 & 60 & 1.76 & 2.34 & 0.84 & 11.81 & 0.62 & -3.01 & & yes \\
\hline & Jan-93 & Dec-97 & 60 & 2.66 & 2.85 & 0.72 & 8.29 & 0.43 & -2.55 & -1.76 & yes \\
\hline & Jan-94 & Dec-98 & 55 & 4.99 & 3.83 & 0.50 & 4.37 & 0.41 & -2.25 & -1.61 & yes \\
\hline Mozambique & Jan-94 & Dec-98 & 60 & 8.50 & 3.32 & 0.40 & 7.28 & 0.34 & -2.31 & -2.60 & no \\
\hline & Jan-95 & Dec-99 & 60 & 7.06 & 3.70 & 0.39 & 8.26 & 0.32 & -2.34 & -2.59 & no \\
\hline & Jan-96 & Dec-00 & 60 & 9.08 & 5.62 & 0.23 & 3.82 & 0.23 & -2.03 & & no \\
\hline & Jan-97 & Dec-01 & 60 & -5.83 & -3.59 & 1.56 & 12.55 & 0.62 & -2.97 & & yes \\
\hline & Jan-98 & Dec-02 & 60 & 22.68 & 2.71 & -1.08 & -1.31 & 0.03 & -0.39 & -1.20 & no \\
\hline & Jan-99 & Dec-03 & 60 & 12.54 & 25.21 & 0.00 & $\# \mathrm{~N} / \mathrm{A}$ & 0.02 & -1.06 & -1.21 & no \\
\hline & Jan-00 & Dec-04 & 60 & 12.95 & 29.35 & 0.00 & \#N/A & 0.03 & -0.81 & -1.09 & no \\
\hline & Jan-01 & Dec-05 & 60 & 12.61 & 25.74 & 0.00 & \#N/A & 0.02 & 0.23 & -0.84 & no \\
\hline & Jan-91 & Dec-95 & 52 & 0.10 & 0.17 & 0.62 & 18.22 & 0.99 & -3.92 & & yes \\
\hline Namibia & Jan-92 & Dec-96 & 60 & -3.02 & -2.84 & 0.83 & 12.99 & 0.45 & -3.07 & -0.89 & yes \\
\hline & Jan-93 & Dec-97 & 60 & -7.64 & -6.25 & 1.14 & 15.28 & 0.59 & -2.96 & -2.21 & yes \\
\hline & Jan-94 & Dec-98 & 60 & -3.09 & -2.62 & 0.87 & 12.57 & 0.40 & -2.48 & & yes \\
\hline & Jan-95 & Dec-99 & 60 & 2.32 & 2.75 & 0.57 & 11.49 & 0.23 & -2.43 & & no \\
\hline & Jan-96 & Dec-00 & 60 & -0.32 & -0.68 & 0.74 & 25.10 & 0.32 & -2.24 & -3.29 & yes \\
\hline & Jan-97 & Dec-01 & 60 & -0.93 & -2.32 & 0.77 & 28.47 & 0.34 & -2.38 & -2.74 & no \\
\hline & Jan-98 & Dec-02 & 60 & -0.90 & -2.35 & 0.76 & 26.94 & 0.37 & -2.65 & -2.76 & no \\
\hline & Jan-99 & Dec-03 & 60 & -0.75 & -1.10 & 0.78 & 13.50 & 0.24 & -1.48 & -0.77 & no \\
\hline & Jan-00 & Dec-04 & 60 & 3.06 & 5.73 & 0.42 & 8.28 & 0.21 & -1.83 & -1.56 & no \\
\hline & Jan-01 & Dec-05 & 60 & 3.09 & 7.94 & 0.43 & 10.79 & 0.23 & -2.10 & -2.30 & no \\
\hline South Africa & Jan-90 & Dec-94 & 60 & -5.91 & -9.26 & 1.35 & 32.39 & 0.35 & -2.17 & & no \\
\hline & Jan-91 & Dec-95 & 60 & -4.30 & -6.29 & 1.23 & 26.15 & 0.45 & -2.63 & & yes \\
\hline
\end{tabular}


Table B-7: Engle-Granger cointegration analysis

\begin{tabular}{|c|c|c|c|c|c|c|c|c|c|c|c|}
\hline \multirow[t]{2}{*}{ Country } & \multicolumn{2}{|c|}{ Rolling sample } & \multirow[t]{2}{*}{ Obs } & \multicolumn{2}{|c|}{ Intercept } & \multicolumn{2}{|c|}{ Slope } & \multirow[t]{2}{*}{$\overline{\mathrm{DW}}$} & \multirow[t]{2}{*}{$\overline{\mathrm{DF}}$} & \multirow[t]{2}{*}{$\overline{\mathrm{ADF}}$} & \multirow[t]{2}{*}{ Coint? } \\
\hline & From & To & & Coeff & t-stat & Coeff & t-stat & & & & \\
\hline \multirow[t]{10}{*}{ South Africa } & Jan-92 & Dec-96 & 60 & -2.03 & -2.95 & 1.06 & 21.93 & 0.62 & -3.36 & & yes \\
\hline & Jan-93 & Dec-97 & 60 & -1.37 & -2.95 & 1.01 & 31.86 & 0.87 & -4.35 & & yes \\
\hline & Jan-94 & Dec-98 & 60 & 0.40 & 0.68 & 0.89 & 23.95 & 0.98 & -4.45 & & yes \\
\hline & Jan-95 & Dec-99 & 60 & 0.01 & 0.01 & 0.90 & 19.05 & 0.77 & -3.66 & & yes \\
\hline & Jan-96 & Dec-00 & 60 & -2.80 & -4.65 & 1.06 & 27.73 & 0.75 & -3.73 & & yes \\
\hline & Jan-97 & Dec-01 & 60 & -1.75 & -3.91 & 0.98 & 32.74 & 0.84 & -3.92 & & yes \\
\hline & Jan-98 & Dec-02 & 60 & -0.99 & -2.45 & 0.92 & 32.05 & 0.94 & -4.33 & & yes \\
\hline & Jan-99 & Dec-03 & 60 & 0.51 & 1.14 & 0.80 & 22.25 & 0.51 & -2.90 & & yes \\
\hline & Jan-00 & Dec-04 & 60 & 0.39 & 1.00 & 0.81 & 22.68 & 0.37 & -2.73 & & no \\
\hline & Jan-01 & Dec-05 & 60 & -0.04 & -0.18 & 0.86 & 40.13 & 0.76 & -3.70 & & yes \\
\hline \multirow[t]{12}{*}{ Seychelles } & Jan-90 & Dec-94 & 60 & -3.24 & -1.71 & 0.94 & 6.65 & 0.43 & -1.84 & & yes \\
\hline & Jan-91 & Dec-95 & 60 & 2.72 & 1.87 & 0.50 & 4.53 & 0.26 & -2.02 & & no \\
\hline & Jan-92 & Dec-96 & 60 & 10.96 & 8.77 & -0.12 & -1.26 & 0.27 & -1.95 & & no \\
\hline & Jan-93 & Dec-97 & 60 & 9.88 & 11.51 & -0.05 & -0.67 & 0.27 & -2.16 & & no \\
\hline & Jan-94 & Dec-98 & 60 & 4.50 & 8.78 & 0.39 & 8.79 & 0.22 & -1.82 & & no \\
\hline & Jan-95 & Dec-99 & 60 & 2.18 & 7.37 & 0.60 & 21.17 & 0.37 & -2.42 & & no \\
\hline & Jan-96 & Dec-00 & 60 & 1.15 & 5.20 & 0.72 & 29.48 & 0.67 & -3.82 & & yes \\
\hline & Jan-97 & Dec-01 & 60 & 0.98 & 5.75 & 0.74 & 32.86 & 0.87 & -4.02 & & yes \\
\hline & Jan-98 & Dec-02 & 60 & 1.13 & 4.97 & 0.71 & 19.57 & 0.90 & -4.08 & -3.92 & yes \\
\hline & Jan-99 & Dec-03 & 60 & -4.64 & -4.22 & 1.73 & 8.54 & 0.95 & -4.80 & -5.31 & yes \\
\hline & Jan-00 & Dec-04 & 60 & 1.29 & 3.49 & 0.62 & 8.57 & 0.65 & -2.80 & -1.51 & yes \\
\hline & Jan-01 & Dec-05 & 60 & 1.56 & 4.23 & 0.57 & 7.32 & 0.51 & -2.77 & & yes \\
\hline \multirow[t]{12}{*}{ Swaziland } & Jan-90 & Dec-94 & 60 & -10.85 & -17.01 & 1.65 & 31.26 & 0.39 & -1.66 & & yes \\
\hline & Jan-91 & Dec-95 & 60 & -1.76 & -1.47 & 0.88 & 9.21 & 0.03 & -0.13 & & no \\
\hline & Jan-92 & Dec-96 & 60 & -0.33 & -0.46 & 0.74 & 13.65 & 0.05 & -2.21 & & no \\
\hline & Jan-93 & Dec-97 & 60 & -1.39 & -5.83 & 0.80 & 46.69 & 0.19 & -2.95 & & no \\
\hline & Jan-94 & Dec-98 & 60 & -2.41 & -10.55 & 0.86 & 56.72 & 0.25 & -1.98 & & no \\
\hline & Jan-95 & Dec-99 & 60 & -4.43 & -19.92 & 0.99 & 69.14 & 1.00 & -5.36 & -3.81 & yes \\
\hline & Jan-96 & Dec-00 & 60 & -4.37 & -43.42 & 0.99 & 147.41 & 1.36 & -5.77 & & yes \\
\hline & Jan-97 & Dec-01 & 60 & -3.79 & -29.32 & 0.95 & 103.44 & 0.59 & -2.87 & & yes \\
\hline & Jan-98 & Dec-02 & 60 & -3.63 & -26.44 & 0.94 & 90.08 & 0.60 & -3.18 & & yes \\
\hline & Jan-99 & Dec-03 & 60 & -2.92 & -13.01 & 0.89 & 47.69 & 0.33 & -1.99 & -1.04 & no \\
\hline & Jan-00 & Dec-04 & 60 & -1.55 & -7.29 & 0.77 & 38.91 & 0.07 & -1.47 & -1.36 & no \\
\hline & Jan-01 & Dec-05 & 60 & -1.57 & -13.58 & 0.78 & 67.92 & 0.15 & -2.68 & -2.98 & no \\
\hline \multirow[t]{8}{*}{ Tanzania } & Jan-94 & Dec-98 & 54 & 1.72 & 1.68 & 0.45 & 14.03 & 0.55 & -3.09 & & yes \\
\hline & Jan-95 & Dec-99 & 60 & 0.70 & 0.90 & 0.48 & 16.92 & 0.59 & -3.84 & -4.94 & yes \\
\hline & Jan-96 & Dec-00 & 60 & -1.67 & -1.51 & 0.59 & 9.79 & 0.36 & -3.02 & -4.70 & yes \\
\hline & Jan-97 & Dec-01 & 60 & 2.66 & 4.48 & 0.30 & 7.75 & 0.36 & -3.91 & -3.97 & yes \\
\hline & Jan-98 & Dec-02 & 60 & 0.71 & 1.72 & 0.40 & 14.07 & 0.54 & -2.87 & & yes \\
\hline & Jan-99 & Dec-03 & 60 & -0.47 & -0.82 & 0.47 & 10.28 & 0.28 & -2.19 & & no \\
\hline & Jan-00 & Dec-04 & 60 & -0.36 & -0.56 & 0.42 & 7.98 & 0.28 & -2.08 & -1.48 & no \\
\hline & Jan-01 & Dec-05 & 60 & 2.51 & 5.21 & 0.13 & 3.19 & 0.42 & -2.84 & & yes \\
\hline Zambia & Jan-92 & Dec-96 & 60 & 3.54 & 1.59 & 0.83 & 23.28 & 0.51 & -2.90 & & yes \\
\hline & Jan-93 & Dec-97 & 60 & 6.59 & 3.25 & 0.78 & 23.16 & 0.42 & -2.65 & & yes \\
\hline & Jan-94 & Dec-98 & 52 & 8.56 & 3.70 & 0.69 & 13.10 & 0.34 & -2.15 & & no \\
\hline & Jan-95 & Dec-99 & 41 & 9.94 & 2.93 & 0.66 & 7.12 & 0.22 & -1.62 & -1.61 & no \\
\hline & Jan-96 & Dec-00 & 41 & 4.78 & 1.40 & 0.79 & 7.84 & 0.21 & -1.54 & -1.49 & no \\
\hline & Jan-97 & Dec-01 & 41 & 15.24 & 3.37 & 0.31 & 2.13 & 0.11 & -1.65 & -1.94 & no \\
\hline & Jan-98 & Dec-02 & 41 & 8.74 & 5.92 & 0.41 & 8.70 & 0.20 & -0.74 & & no \\
\hline & Jan-99 & Dec-03 & 49 & 17.07 & 18.44 & 0.17 & 5.65 & 0.19 & -1.54 & & no \\
\hline & Jan-00 & Dec-04 & 60 & 8.45 & 9.55 & 0.43 & 13.93 & 0.30 & -1.73 & & no \\
\hline & Jan-01 & Dec-05 & 60 & 6.10 & 7.03 & 0.50 & 15.26 & 0.26 & -1.89 & & no \\
\hline Zimbabwe & Jan-90 & Dec-94 & 60 & -0.45 & -0.43 & 1.01 & 22.34 & 0.53 & -2.93 & & yes \\
\hline & Jan-91 & Dec-95 & 60 & 0.78 & 0.40 & 0.94 & 12.75 & 0.41 & -2.43 & & yes \\
\hline & Jan-92 & Dec-96 & 60 & 9.67 & 1.29 & 0.59 & 2.26 & 0.20 & -1.14 & & no \\
\hline & Jan-93 & Dec-97 & 60 & -34.25 & -4.02 & 2.06 & 6.90 & 0.27 & -2.36 & -2.69 & no \\
\hline & Jan-94 & Dec-98 & 60 & -15.57 & -4.39 & 1.36 & 11.31 & 0.44 & -2.18 & & yes \\
\hline & Jan-95 & Dec-99 & 60 & 5.43 & 4.34 & 0.62 & 17.94 & 0.25 & -2.18 & & no \\
\hline & Jan-96 & Dec-00 & 60 & 3.01 & 2.93 & 0.68 & 30.07 & 0.55 & -3.01 & -3.11 & yes \\
\hline
\end{tabular}


Table B-7: Engle-Granger cointegration analysis

\begin{tabular}{|c|c|c|c|c|c|c|c|c|c|c|c|}
\hline \multirow[t]{2}{*}{ Country } & \multicolumn{2}{|c|}{ Rolling sample } & \multirow[t]{2}{*}{ Obs } & \multicolumn{2}{|c|}{ Intercept } & \multicolumn{2}{|c|}{ Slope } & \multirow[t]{2}{*}{ DW } & \multirow[t]{2}{*}{$\mathrm{DF}$} & \multirow[t]{2}{*}{ ADF } & \multirow[t]{2}{*}{ Coint? } \\
\hline & From & To & & Coeff & t-stat & Coeff & t-stat & & & & \\
\hline \multirow[t]{5}{*}{ Zimbabwe } & Jan-97 & Dec-01 & 60 & 7.37 & 1.62 & 0.48 & 5.30 & 0.09 & -0.54 & -1.83 & no \\
\hline & Jan-98 & Dec-02 & 60 & 10.06 & 1.41 & 0.37 & 2.88 & 0.08 & -1.12 & -1.52 & no \\
\hline & Jan-99 & Dec-03 & 60 & -6.86 & -1.79 & 0.62 & 11.29 & 0.28 & -2.24 & & no \\
\hline & Jan-00 & Dec-04 & 60 & 0.80 & 0.15 & 0.46 & 10.96 & 1.53 & -6.06 & & yes \\
\hline & Jan-01 & Dec-05 & 60 & 2.85 & 0.54 & 0.39 & 12.68 & 1.46 & -5.74 & & yes \\
\hline \multicolumn{12}{|c|}{ Panel D: Cointegration of national deposit rate and South African central bank policy rate } \\
\hline \multirow{12}{*}{ Botswana } & Jan-90 & Dec-94 & 60 & 20.79 & 11.13 & -0.66 & -5.40 & 0.20 & -2.13 & -2.39 & no \\
\hline & Jan-91 & Dec-95 & 60 & 12.65 & 8.38 & -0.08 & -0.73 & 0.33 & -2.14 & -1.33 & no \\
\hline & Jan-92 & Dec-96 & 60 & 15.99 & 8.45 & -0.34 & -2.54 & 0.28 & -2.11 & -1.73 & no \\
\hline & Jan-93 & Dec-97 & 60 & 18.33 & 13.49 & -0.53 & -5.72 & 0.43 & -3.05 & -2.69 & yes \\
\hline & Jan-94 & Dec-98 & 60 & 13.37 & 23.69 & -0.24 & -6.77 & 1.50 & -6.81 & -5.65 & yes \\
\hline & Jan-95 & Dec-99 & 60 & 11.45 & 20.64 & -0.13 & -3.90 & 1.12 & -4.72 & -3.65 & yes \\
\hline & Jan-96 & Dec-00 & 60 & 10.47 & 32.12 & -0.08 & -3.88 & 0.18 & -1.43 & -1.83 & no \\
\hline & Jan-97 & Dec-01 & 60 & 11.06 & 44.53 & -0.12 & -7.24 & 0.22 & -2.08 & -2.47 & no \\
\hline & Jan-98 & Dec-02 & 60 & 11.71 & 37.35 & -0.16 & -7.26 & 0.16 & -0.81 & -1.52 & no \\
\hline & Jan-99 & Dec-03 & 60 & 10.70 & 24.27 & -0.07 & -2.06 & 0.15 & -1.43 & & no \\
\hline & Jan-00 & Dec-04 & 60 & 9.26 & 29.28 & 0.06 & 2.24 & 0.32 & -2.70 & & no \\
\hline & Jan-01 & Dec-05 & 60 & 8.43 & 44.92 & 0.15 & 8.19 & 0.59 & -3.17 & & yes \\
\hline \multirow[t]{12}{*}{ Lesotho } & Jan-90 & Dec-94 & 60 & -3.98 & -5.24 & 0.96 & 19.43 & 0.55 & -2.67 & -2.95 & yes \\
\hline & Jan-91 & Dec-95 & 60 & -4.44 & -2.75 & 1.05 & 9.43 & 0.21 & -1.62 & -1.81 & no \\
\hline & Jan-92 & Dec-96 & 60 & -6.28 & -3.11 & 1.19 & 8.41 & 0.25 & -1.66 & -2.02 & no \\
\hline & Jan-93 & Dec-97 & 60 & -3.17 & -1.83 & 0.97 & 8.17 & 0.19 & -1.67 & & no \\
\hline & Jan-94 & Dec-98 & 60 & 7.22 & 4.65 & 0.27 & 2.74 & 0.16 & -1.90 & -2.25 & no \\
\hline & Jan-95 & Dec-99 & 60 & 7.58 & 3.46 & 0.23 & 1.68 & 0.14 & -0.72 & & no \\
\hline & Jan-96 & Dec-00 & 60 & -2.93 & -1.83 & 0.80 & 7.92 & 0.16 & -2.15 & & no \\
\hline & Jan-97 & Dec-01 & 60 & -3.62 & -4.17 & 0.79 & 13.69 & 0.26 & -2.08 & & no \\
\hline & Jan-98 & Dec-02 & 60 & -2.67 & -4.77 & 0.68 & 17.10 & 0.53 & -3.51 & -4.75 & yes \\
\hline & Jan-99 & Dec-03 & 60 & -1.01 & -1.64 & 0.53 & 10.70 & 0.66 & -4.07 & -2.40 & yes \\
\hline & Jan-00 & Dec-04 & 60 & 2.49 & 12.45 & 0.22 & 12.14 & 0.73 & -3.86 & & yes \\
\hline & Jan-01 & Dec-05 & 60 & 2.28 & 9.24 & 0.24 & 9.99 & 1.94 & -7.43 & & yes \\
\hline Mozambique & Jan-94 & Dec-98 & 60 & 71.00 & 7.92 & -2.95 & -5.22 & 0.29 & -2.34 & & no \\
\hline & Jan-95 & Dec-99 & 60 & 42.83 & 3.61 & -1.44 & -1.97 & 0.15 & -1.54 & & no \\
\hline & Jan-96 & Dec-00 & 60 & 4.64 & 0.73 & 0.59 & 1.48 & 0.18 & -1.66 & & no \\
\hline & Jan-97 & Dec-01 & 60 & 10.62 & 2.15 & 0.18 & 0.55 & 0.14 & -2.39 & & no \\
\hline & Jan-98 & Dec-02 & 60 & 21.81 & 11.28 & -0.74 & -5.35 & 0.06 & -0.50 & -0.87 & no \\
\hline & Jan-99 & Dec-03 & 60 & 20.68 & 6.99 & -0.67 & -2.79 & 0.04 & -0.07 & -1.22 & no \\
\hline & Jan-00 & Dec-04 & 60 & 6.00 & 2.60 & 0.64 & 3.06 & 0.05 & -1.48 & -2.07 & no \\
\hline & Jan-01 & Dec-05 & 60 & 1.12 & 0.77 & 1.16 & 8.13 & 0.10 & -1.23 & -3.04 & no \\
\hline Namibia & Jan-91 & Dec-95 & 60 & 0.38 & 0.94 & 0.72 & 25.71 & 1.22 & -4.88 & & yes \\
\hline & Jan-92 & Dec-96 & 60 & -0.57 & -0.95 & 0.79 & 19.09 & 0.84 & -4.03 & & yes \\
\hline & Jan-93 & Dec-97 & 60 & -0.80 & -1.62 & 0.81 & 24.09 & 0.91 & -4.16 & & yes \\
\hline & Jan-94 & Dec-98 & 60 & 2.70 & 4.26 & 0.57 & 14.28 & 0.40 & -2.56 & & yes \\
\hline & Jan-95 & Dec-99 & 60 & 3.25 & 4.73 & 0.54 & 12.80 & 0.34 & -2.14 & -2.87 & yes \\
\hline & Jan-96 & Dec-00 & 60 & -0.35 & -0.47 & 0.75 & 15.73 & 0.22 & -1.98 & -2.22 & no \\
\hline & Jan-97 & Dec-01 & 60 & -1.27 & -2.32 & 0.78 & 21.28 & 0.29 & -2.34 & -3.34 & yes \\
\hline & Jan-98 & Dec-02 & 60 & -1.03 & -2.18 & 0.75 & 22.19 & 0.38 & -2.78 & -3.13 & yes \\
\hline & Jan-99 & Dec-03 & 60 & -0.71 & -0.97 & 0.74 & 12.50 & 0.24 & -1.54 & -1.40 & no \\
\hline & Jan-00 & Dec-04 & 60 & 3.30 & 6.50 & 0.38 & 8.24 & 0.21 & -1.90 & -1.52 & no \\
\hline & Jan-01 & Dec-05 & 60 & 3.39 & 9.36 & 0.38 & 10.80 & 0.23 & -2.17 & -1.95 & no \\
\hline Swaziland & Jan-90 & Dec-94 & 60 & 3.35 & 3.79 & 0.38 & 6.54 & 0.08 & -1.42 & -2.15 & no \\
\hline & Jan-91 & Dec-95 & 60 & -0.63 & -1.07 & 0.68 & 16.69 & 0.38 & -3.30 & -2.81 & yes \\
\hline & Jan-92 & Dec-96 & 60 & -4.06 & -7.87 & 0.94 & 26.03 & 0.96 & -4.90 & -4.73 & yes \\
\hline & Jan-93 & Dec-97 & 60 & -4.97 & -14.48 & 1.01 & 42.73 & 1.46 & -5.78 & -3.78 & yes \\
\hline & Jan-94 & Dec-98 & 60 & -0.24 & -0.35 & 0.68 & 15.66 & 0.35 & -2.36 & -2.65 & no \\
\hline & Jan-95 & Dec-99 & 60 & 0.67 & 0.82 & 0.63 & 12.68 & 0.41 & -2.65 & & yes \\
\hline & Jan-96 & Dec-00 & 60 & -1.74 & -2.41 & 0.77 & 16.90 & 0.32 & -2.15 & & no \\
\hline & Jan-97 & Dec-01 & 60 & -2.13 & -3.78 & 0.78 & 20.80 & 0.31 & -2.35 & & no \\
\hline & Jan-98 & Dec-02 & 60 & -1.28 & -2.66 & 0.72 & 20.83 & 0.36 & -2.50 & & no \\
\hline & Jan-99 & Dec-03 & 60 & -3.19 & -6.13 & 0.89 & 21.04 & 0.33 & -2.08 & -1.27 & no \\
\hline
\end{tabular}


Table B-7: Engle-Granger cointegration analysis

\begin{tabular}{|c|c|c|c|c|c|c|c|c|c|c|c|}
\hline \multirow[t]{2}{*}{ Country } & \multicolumn{2}{|c|}{ Rolling sample } & \multirow[t]{2}{*}{ Obs } & \multicolumn{2}{|c|}{ Intercept } & \multicolumn{2}{|c|}{ Slope } & \multirow[t]{2}{*}{ DW } & \multirow[t]{2}{*}{$\overline{\mathrm{DF}}$} & \multirow[t]{2}{*}{ ADF } & \multirow[t]{2}{*}{ Coint? } \\
\hline & From & To & & Coeff & t-stat & Coeff & t-stat & & & & \\
\hline \multirow[t]{2}{*}{ Swaziland } & Jan-00 & Dec-04 & 60 & -0.93 & -2.25 & 0.69 & 18.47 & 0.04 & -1.24 & -1.47 & no \\
\hline & Jan-01 & Dec-05 & 60 & -1.27 & -5.34 & 0.74 & 31.69 & 0.08 & -2.78 & -3.31 & yes \\
\hline \multirow[t]{12}{*}{ Zambia } & Jan-90 & Dec-94 & 60 & 154.30 & 9.91 & -7.04 & -6.93 & 0.11 & 0.17 & -1.33 & no \\
\hline & Jan-91 & Dec-95 & 60 & 139.68 & 6.60 & -6.30 & -4.34 & 0.09 & -1.13 & -2.00 & no \\
\hline & Jan-92 & Dec-96 & 60 & 130.44 & 5.21 & -5.61 & -3.20 & 0.09 & -1.09 & -2.13 & no \\
\hline & Jan-93 & Dec-97 & 60 & 135.79 & 6.07 & -6.07 & -3.96 & 0.08 & -1.09 & -2.32 & no \\
\hline & Jan-94 & Dec-98 & 60 & 82.03 & 7.49 & -3.12 & -4.51 & 0.10 & -1.84 & -2.75 & no \\
\hline & Jan-95 & Dec-99 & 60 & 42.23 & 4.00 & -0.88 & -1.36 & 0.05 & -1.12 & -1.30 & no \\
\hline & Jan-96 & Dec-00 & 60 & 23.75 & 2.86 & 0.15 & 0.28 & 0.04 & -1.01 & -2.41 & no \\
\hline & Jan-97 & Dec-01 & 60 & 24.65 & 5.51 & -0.16 & -0.54 & 0.08 & -3.27 & -3.39 & yes \\
\hline & Jan-98 & Dec-02 & 60 & 33.17 & 24.52 & -0.96 & -9.96 & 0.14 & -2.28 & -4.76 & yes \\
\hline & Jan-99 & Dec-03 & 60 & 26.78 & 22.37 & -0.41 & -4.18 & 0.18 & -1.77 & -1.27 & no \\
\hline & Jan-00 & Dec-04 & 60 & 2.26 & 0.94 & 1.64 & 7.55 & 0.12 & -1.11 & -2.93 & no \\
\hline & Jan-01 & Dec-05 & 60 & -1.67 & -0.89 & 2.01 & 10.94 & 0.15 & -1.50 & -3.03 & no \\
\hline
\end{tabular}

Note: The following test statistics are reported: Durbin Watson (DW), Dickey Fuller (DF), Augmented Dickey Fuller with optimal lag length selected by AIC criteria based on all models up to 4 lags (ADF). The critical values at the 1\%, 5\%, and $10 \%$ level for 100 observations are as follows: 0.511, 0.386, and 0.322 for DW, 4.07, 3.37, and 3.03 for DF, 3.77, 3.17, and 2.84 for ADF. Cointegration is considered to exist if at least 2 test statistics are significant at $10 \%$ level or 1 test statistic at $5 \%$ level. For details on the methodology see Engle and Granger (1987). 
Table B-8: Kremers-Ericsson-Dolado cointegration analysis

\begin{tabular}{|c|c|c|c|c|c|c|}
\hline \multirow[t]{2}{*}{ Country } & \multicolumn{2}{|c|}{ Rolling sample } & \multirow[t]{2}{*}{ Obs } & \multicolumn{2}{|c|}{$\mathrm{ECT}_{\mathrm{t}-1}$} & \multirow[t]{2}{*}{ Coint? } \\
\hline & From & To & & Coeff & t-stat & \\
\hline \multicolumn{7}{|c|}{ Panel A: Cointegration of national lending rate and national central bank policy rate } \\
\hline \multirow[t]{7}{*}{ Angola } & Jan-95 & Dec-99 & 60 & -0.33 & -3.12 & yes \\
\hline & Jan-96 & Dec-00 & 60 & -0.22 & -2.36 & yes \\
\hline & Jan-97 & Dec-01 & 60 & -0.35 & -3.74 & yes \\
\hline & Jan-98 & Dec-02 & 60 & -0.31 & -3.52 & yes \\
\hline & Jan-99 & Dec-03 & 60 & -0.37 & -3.70 & yes \\
\hline & Jan-00 & Dec-04 & 60 & -0.53 & -3.92 & yes \\
\hline & Jan-01 & Dec-05 & 60 & -0.43 & -3.36 & yes \\
\hline \multirow[t]{12}{*}{ Botswana } & Jan-90 & Dec-94 & 60 & -0.28 & -3.46 & yes \\
\hline & Jan-91 & Dec-95 & 60 & -0.35 & -3.74 & yes \\
\hline & Jan-92 & Dec-96 & 60 & -0.27 & -3.25 & yes \\
\hline & Jan-93 & Dec-97 & 60 & -0.29 & -2.74 & yes \\
\hline & Jan-94 & Dec-98 & 60 & -0.26 & -3.53 & yes \\
\hline & Jan-95 & Dec-99 & 60 & -0.20 & -3.09 & yes \\
\hline & Jan-96 & Dec-00 & 60 & -0.39 & -4.91 & yes \\
\hline & Jan-97 & Dec-01 & 60 & -0.38 & -5.27 & yes \\
\hline & Jan-98 & Dec-02 & 60 & -0.42 & -5.29 & yes \\
\hline & Jan-99 & Dec-03 & 60 & -0.44 & -5.18 & yes \\
\hline & Jan-00 & Dec-04 & 60 & -0.37 & -4.03 & yes \\
\hline & Jan-01 & Dec-05 & 60 & -0.88 & -6.78 & yes \\
\hline \multirow[t]{6}{*}{ DR Congo } & Jan-94 & Dec-98 & 60 & -0.04 & -1.25 & no \\
\hline & Jan-95 & Dec-99 & 60 & -0.21 & -4.04 & yes \\
\hline & Jan-96 & Dec-00 & 60 & -0.47 & -5.39 & yes \\
\hline & Jan-97 & Dec-01 & 60 & -0.56 & -7.11 & yes \\
\hline & Jan-98 & Dec-02 & 60 & -0.62 & -5.85 & yes \\
\hline & Jan-99 & Dec-03 & 53 & -0.62 & -5.26 & yes \\
\hline \multirow[t]{12}{*}{ Lesotho } & Jan-90 & Dec-94 & 60 & -0.35 & -2.75 & yes \\
\hline & Jan-91 & Dec-95 & 60 & -0.66 & -3.65 & yes \\
\hline & Jan-92 & Dec-96 & 60 & -0.75 & -4.05 & yes \\
\hline & Jan-93 & Dec-97 & 60 & -0.82 & -4.36 & yes \\
\hline & Jan-94 & Dec-98 & 60 & -0.27 & -2.45 & yes \\
\hline & Jan-95 & Dec-99 & 60 & -0.15 & -1.45 & no \\
\hline & Jan-96 & Dec-00 & 60 & -0.13 & -1.87 & yes \\
\hline & Jan-97 & Dec-01 & 60 & -0.11 & -1.68 & yes \\
\hline & Jan-98 & Dec-02 & 60 & -0.12 & -1.76 & yes \\
\hline & Jan-99 & Dec-03 & 60 & -0.11 & -1.48 & no \\
\hline & Jan-00 & Dec-04 & 60 & 0.00 & 0.10 & no \\
\hline & Jan-01 & Dec-05 & 60 & 0.02 & 0.63 & no \\
\hline \multirow[t]{11}{*}{ Madagascar } & Jan-91 & Dec-95 & 60 & -0.27 & -2.75 & yes \\
\hline & Jan-92 & Dec-96 & 60 & -0.23 & -3.20 & yes \\
\hline & Jan-93 & Dec-97 & 60 & -0.19 & -3.18 & yes \\
\hline & Jan-94 & Dec-98 & 60 & -0.31 & -3.94 & yes \\
\hline & Jan-95 & Dec-99 & 60 & -0.35 & -5.42 & yes \\
\hline & Jan-96 & Dec-00 & 60 & -0.15 & -2.83 & yes \\
\hline & Jan-97 & Dec-01 & 60 & -0.07 & -1.80 & yes \\
\hline & Jan-98 & Dec-02 & 60 & -0.04 & -0.88 & no \\
\hline & Jan-99 & Dec-03 & 60 & -0.12 & -2.08 & yes \\
\hline & Jan-00 & Dec-04 & 60 & -0.16 & -3.50 & yes \\
\hline & Jan-01 & Dec-05 & 60 & -0.18 & -2.34 & yes \\
\hline \multirow[t]{10}{*}{ Malawi } & Jan-90 & Dec-94 & 60 & -0.16 & -2.44 & yes \\
\hline & Jan-91 & Dec-95 & 60 & -0.29 & -3.22 & yes \\
\hline & Jan-92 & Dec-96 & 60 & -0.29 & -3.29 & yes \\
\hline & Jan-93 & Dec-97 & 60 & -0.28 & -3.14 & yes \\
\hline & Jan-94 & Dec-98 & 60 & -0.28 & -3.12 & yes \\
\hline & Jan-95 & Dec-99 & 60 & -0.32 & -4.13 & yes \\
\hline & Jan-96 & Dec-00 & 60 & -0.18 & -2.43 & yes \\
\hline & Jan-97 & Dec-01 & 60 & -0.25 & -2.82 & yes \\
\hline & Jan-98 & Dec-02 & 60 & -0.42 & -4.81 & yes \\
\hline & Jan-99 & Dec-03 & 60 & -0.63 & -4.61 & yes \\
\hline
\end{tabular}


Table B-8: Kremers-Ericsson-Dolado cointegration analysis

\begin{tabular}{|c|c|c|c|c|c|c|}
\hline \multirow[t]{2}{*}{ Country } & \multicolumn{2}{|c|}{ Rolling sample } & \multirow[t]{2}{*}{ Obs } & \multicolumn{2}{|c|}{$\mathrm{ECT}_{\mathrm{t}-1}$} & \multirow[t]{2}{*}{ Coint? } \\
\hline & From & To & & Coeff & t-stat & \\
\hline \multirow[t]{2}{*}{ Malawi } & Jan-00 & Dec-04 & 60 & -0.42 & -3.80 & yes \\
\hline & Jan-01 & Dec-05 & 60 & -0.42 & -4.42 & yes \\
\hline \multirow[t]{5}{*}{ Mauritius } & Jan-90 & Dec-94 & 60 & 0.00 & -0.05 & no \\
\hline & Jan-91 & Dec-95 & 60 & -0.02 & -0.34 & no \\
\hline & Jan-92 & Dec-96 & 60 & -0.12 & -1.84 & yes \\
\hline & Jan-93 & Dec-97 & 60 & -0.37 & -3.14 & yes \\
\hline & Jan-94 & Dec-98 & 55 & -0.40 & -3.22 & yes \\
\hline \multirow[t]{5}{*}{ Mozambique } & Jan-97 & Dec-01 & 53 & -0.30 & -3.07 & yes \\
\hline & Jan-98 & Dec-02 & 60 & -0.01 & -0.31 & no \\
\hline & Jan-99 & Dec-03 & 60 & -0.04 & -1.32 & no \\
\hline & Jan-00 & Dec-04 & 60 & -0.05 & -1.48 & no \\
\hline & Jan-01 & Dec-05 & 60 & -0.04 & -1.10 & no \\
\hline \multirow[t]{10}{*}{ Namibia } & Jan-92 & Dec-96 & 60 & -0.66 & -5.84 & yes \\
\hline & Jan-93 & Dec-97 & 60 & -0.74 & -5.74 & yes \\
\hline & Jan-94 & Dec-98 & 60 & -0.57 & -4.70 & yes \\
\hline & Jan-95 & Dec-99 & 60 & -0.53 & -4.60 & yes \\
\hline & Jan-96 & Dec-00 & 60 & -0.51 & -4.36 & yes \\
\hline & Jan-97 & Dec-01 & 60 & -0.62 & -5.19 & yes \\
\hline & Jan-98 & Dec-02 & 60 & -0.67 & -6.32 & yes \\
\hline & Jan-99 & Dec-03 & 60 & -0.47 & -5.50 & yes \\
\hline & Jan-00 & Dec-04 & 60 & -0.42 & -5.10 & yes \\
\hline & Jan-01 & Dec-05 & 60 & -0.48 & -5.36 & yes \\
\hline \multirow[t]{12}{*}{ South Africa } & Jan-90 & Dec-94 & 60 & -0.51 & -6.21 & yes \\
\hline & Jan-91 & Dec-95 & 60 & -0.89 & -10.69 & yes \\
\hline & Jan-92 & Dec-96 & 60 & -0.95 & -10.08 & yes \\
\hline & Jan-93 & Dec-97 & 60 & -0.90 & -8.51 & yes \\
\hline & Jan-94 & Dec-98 & 60 & -0.68 & -5.85 & yes \\
\hline & Jan-95 & Dec-99 & 60 & -0.82 & -7.97 & yes \\
\hline & Jan-96 & Dec-00 & 60 & -0.90 & -8.84 & yes \\
\hline & Jan-97 & Dec-01 & 60 & -0.74 & -7.46 & yes \\
\hline & Jan-98 & Dec-02 & 60 & -0.77 & -8.27 & yes \\
\hline & Jan-99 & Dec-03 & 60 & -0.57 & -5.83 & yes \\
\hline & Jan-00 & Dec-04 & 60 & -0.23 & -2.79 & yes \\
\hline & Jan-01 & Dec-05 & 60 & -0.06 & -1.43 & no \\
\hline \multirow[t]{12}{*}{ Seychelles } & Jan-90 & Dec-94 & 60 & -0.12 & -2.40 & yes \\
\hline & Jan-91 & Dec-95 & 60 & -0.12 & -1.70 & yes \\
\hline & Jan-92 & Dec-96 & 60 & -0.13 & -1.91 & yes \\
\hline & Jan-93 & Dec-97 & 60 & -0.12 & -1.23 & no \\
\hline & Jan-94 & Dec-98 & 60 & -0.20 & -2.70 & yes \\
\hline & Jan-95 & Dec-99 & 60 & -0.18 & -2.18 & yes \\
\hline & Jan-96 & Dec-00 & 60 & -0.14 & -1.94 & yes \\
\hline & Jan-97 & Dec-01 & 60 & -0.16 & -2.41 & yes \\
\hline & Jan-98 & Dec-02 & 60 & -0.08 & -1.77 & yes \\
\hline & Jan-99 & Dec-03 & 60 & -0.11 & -3.10 & yes \\
\hline & Jan-00 & Dec-04 & 60 & -0.13 & -2.71 & yes \\
\hline & Jan-01 & Dec-05 & 60 & -0.24 & -3.11 & yes \\
\hline \multirow[t]{12}{*}{ Swaziland } & Jan-90 & Dec-94 & 60 & -0.28 & -4.03 & yes \\
\hline & Jan-91 & Dec-95 & 60 & -0.18 & -2.73 & yes \\
\hline & Jan-92 & Dec-96 & 60 & -0.50 & -5.07 & yes \\
\hline & Jan-93 & Dec-97 & 60 & -0.83 & -7.11 & yes \\
\hline & Jan-94 & Dec-98 & 60 & -0.86 & -7.16 & yes \\
\hline & Jan-95 & Dec-99 & 60 & -1.00 & -7.79 & yes \\
\hline & Jan-96 & Dec-00 & 60 & -1.03 & -7.95 & yes \\
\hline & Jan-97 & Dec-01 & 60 & 0.00 & 0.55 & no \\
\hline & Jan-98 & Dec-02 & 60 & 0.00 & 1.12 & no \\
\hline & Jan-99 & Dec-03 & 60 & 0.00 & 1.00 & no \\
\hline & Jan-00 & Dec-04 & 60 & -0.12 & -1.45 & no \\
\hline & Jan-01 & Dec-05 & 60 & -0.08 & -1.34 & no \\
\hline Tanzania & Jan-92 & Dec-96 & 56 & -0.11 & -1.62 & no \\
\hline
\end{tabular}


Table B-8: Kremers-Ericsson-Dolado cointegration analysis

\begin{tabular}{|c|c|c|c|c|c|c|}
\hline \multirow[t]{2}{*}{ Country } & \multicolumn{2}{|c|}{ Rolling sample } & \multirow[t]{2}{*}{ Obs } & \multicolumn{2}{|c|}{$\mathrm{ECT}_{\mathrm{t}-1}$} & \multirow[t]{2}{*}{ Coint? } \\
\hline & From & To & & Coeff & t-stat & \\
\hline \multirow[t]{9}{*}{ Tanzania } & Jan-93 & Dec-97 & 60 & -0.16 & -1.88 & yes \\
\hline & Jan-94 & Dec-98 & 60 & -0.08 & -1.15 & no \\
\hline & Jan-95 & Dec-99 & 60 & -0.07 & -1.68 & yes \\
\hline & Jan-96 & Dec-00 & 60 & -0.11 & -2.47 & yes \\
\hline & Jan-97 & Dec-01 & 60 & -0.10 & -3.28 & yes \\
\hline & Jan-98 & Dec-02 & 60 & -0.13 & -3.61 & yes \\
\hline & Jan-99 & Dec-03 & 60 & -0.05 & -1.17 & no \\
\hline & Jan-00 & Dec-04 & 60 & -0.02 & -0.61 & no \\
\hline & Jan-01 & Dec-05 & 60 & -0.01 & -0.29 & no \\
\hline \multirow[t]{10}{*}{ Zambia } & Jan-92 & Dec-96 & 60 & -0.11 & -2.56 & yes \\
\hline & Jan-93 & Dec-97 & 60 & -0.28 & -3.08 & yes \\
\hline & Jan-94 & Dec-98 & 52 & -0.20 & -2.94 & yes \\
\hline & Jan-95 & Dec-99 & 41 & -0.13 & -3.18 & yes \\
\hline & Jan-96 & Dec-00 & 41 & -0.13 & -2.14 & yes \\
\hline & Jan-97 & Dec-01 & 41 & -0.11 & -1.50 & no \\
\hline & Jan-98 & Dec-02 & 41 & -0.17 & -1.81 & yes \\
\hline & Jan-99 & Dec-03 & 49 & -0.26 & -2.67 & yes \\
\hline & Jan-00 & Dec-04 & 60 & -0.26 & -3.56 & yes \\
\hline & Jan-01 & Dec-05 & 60 & -0.19 & -3.09 & yes \\
\hline \multirow[t]{12}{*}{ Zimbabwe } & Jan-90 & Dec-94 & 60 & -0.15 & -2.41 & yes \\
\hline & Jan-91 & Dec-95 & 60 & -0.18 & -2.55 & yes \\
\hline & Jan-92 & Dec-96 & 60 & -0.18 & -2.72 & yes \\
\hline & Jan-93 & Dec-97 & 60 & -0.68 & -4.65 & yes \\
\hline & Jan-94 & Dec-98 & 60 & -0.35 & -2.54 & yes \\
\hline & Jan-95 & Dec-99 & 60 & -0.19 & -1.88 & yes \\
\hline & Jan-96 & Dec-00 & 60 & -0.03 & -0.60 & no \\
\hline & Jan-97 & Dec-01 & 60 & -0.05 & -1.07 & no \\
\hline & Jan-98 & Dec-02 & 60 & -0.05 & -1.45 & no \\
\hline & Jan-99 & Dec-03 & 60 & 0.03 & 0.37 & no \\
\hline & Jan-00 & Dec-04 & 60 & -0.44 & -3.27 & yes \\
\hline & Jan-01 & Dec-05 & 60 & -0.26 & -2.68 & yes \\
\hline \multicolumn{7}{|c|}{ Panel B: Cointegration of national lending rate and South African central bank policy rate } \\
\hline \multirow{12}{*}{ Botswana } & Jan-90 & Dec-94 & 60 & -0.07 & -1.14 & no \\
\hline & Jan-91 & Dec-95 & 60 & -0.12 & -1.85 & yes \\
\hline & Jan-92 & Dec-96 & 60 & -0.26 & -3.62 & yes \\
\hline & Jan-93 & Dec-97 & 60 & -0.20 & -2.24 & yes \\
\hline & Jan-94 & Dec-98 & 60 & -0.12 & -1.90 & yes \\
\hline & Jan-95 & Dec-99 & 60 & -0.05 & -0.68 & no \\
\hline & Jan-96 & Dec-00 & 60 & 0.01 & 0.23 & no \\
\hline & Jan-97 & Dec-01 & 60 & 0.02 & 0.51 & no \\
\hline & Jan-98 & Dec-02 & 60 & -0.02 & -0.65 & no \\
\hline & Jan-99 & Dec-03 & 60 & -0.06 & -1.87 & yes \\
\hline & Jan-00 & Dec-04 & 60 & -0.10 & -2.40 & yes \\
\hline & Jan-01 & Dec-05 & 60 & -0.17 & -2.61 & yes \\
\hline \multirow[t]{12}{*}{ Lesotho } & Jan-90 & Dec-94 & 60 & -1.02 & -5.51 & yes \\
\hline & Jan-91 & Dec-95 & 60 & -0.82 & -4.90 & yes \\
\hline & Jan-92 & Dec-96 & 60 & -0.87 & -5.40 & yes \\
\hline & Jan-93 & Dec-97 & 60 & -1.06 & -5.73 & yes \\
\hline & Jan-94 & Dec-98 & 60 & -0.82 & -7.90 & yes \\
\hline & Jan-95 & Dec-99 & 60 & -0.37 & -4.08 & yes \\
\hline & Jan-96 & Dec-00 & 60 & -0.35 & -3.71 & yes \\
\hline & Jan-97 & Dec-01 & 60 & -0.33 & -3.40 & yes \\
\hline & Jan-98 & Dec-02 & 60 & -0.56 & -5.58 & yes \\
\hline & Jan-99 & Dec-03 & 60 & -0.40 & -3.12 & yes \\
\hline & Jan-00 & Dec-04 & 60 & -0.13 & -1.99 & yes \\
\hline & Jan-01 & Dec-05 & 60 & -0.13 & -2.04 & yes \\
\hline \multirow[t]{3}{*}{ Mozambique } & Jan-97 & Dec-01 & 53 & -0.25 & -3.21 & yes \\
\hline & Jan-98 & Dec-02 & 60 & -0.01 & -0.30 & no \\
\hline & Jan-99 & Dec-03 & 60 & -0.05 & -1.51 & no \\
\hline
\end{tabular}


Table B-8: Kremers-Ericsson-Dolado cointegration analysis

\begin{tabular}{|c|c|c|c|c|c|c|}
\hline \multirow[t]{2}{*}{ Country } & \multicolumn{2}{|c|}{ Rolling sample } & \multirow[t]{2}{*}{ Obs } & \multicolumn{2}{|c|}{$\mathrm{ECT}_{\mathrm{t}-1}$} & \multirow[t]{2}{*}{ Coint? } \\
\hline & From & To & & Coeff & t-stat & \\
\hline \multirow[t]{2}{*}{ Mozambique } & Jan-00 & Dec-04 & 60 & -0.06 & -1.64 & no \\
\hline & Jan-01 & Dec-05 & 60 & -0.13 & -2.40 & yes \\
\hline \multirow[t]{11}{*}{ Namibia } & Jan-91 & Dec-95 & 60 & -0.37 & -4.51 & yes \\
\hline & Jan-92 & Dec-96 & 60 & -0.72 & -7.41 & yes \\
\hline & Jan-93 & Dec-97 & 60 & -0.88 & -8.07 & yes \\
\hline & Jan-94 & Dec-98 & 60 & -0.88 & -7.55 & yes \\
\hline & Jan-95 & Dec-99 & 60 & -0.86 & -7.03 & yes \\
\hline & Jan-96 & Dec-00 & 60 & -0.67 & -6.20 & yes \\
\hline & Jan-97 & Dec-01 & 60 & -0.55 & -6.06 & yes \\
\hline & Jan-98 & Dec-02 & 60 & -0.46 & -6.09 & yes \\
\hline & Jan-99 & Dec-03 & 60 & -0.43 & -5.73 & yes \\
\hline & Jan-00 & Dec-04 & 60 & -0.50 & -5.93 & yes \\
\hline & Jan-01 & Dec-05 & 60 & -0.57 & -7.13 & yes \\
\hline \multirow[t]{12}{*}{ Swaziland } & Jan-90 & Dec-94 & 60 & -0.08 & -1.80 & yes \\
\hline & Jan-91 & Dec-95 & 60 & -0.04 & -0.76 & no \\
\hline & Jan-92 & Dec-96 & 60 & -0.06 & -1.03 & no \\
\hline & Jan-93 & Dec-97 & 60 & -0.43 & -4.71 & yes \\
\hline & Jan-94 & Dec-98 & 60 & -0.20 & -3.74 & yes \\
\hline & Jan-95 & Dec-99 & 60 & -0.29 & -4.18 & yes \\
\hline & Jan-96 & Dec-00 & 60 & -0.20 & -3.43 & yes \\
\hline & Jan-97 & Dec-01 & 60 & -0.22 & -4.09 & yes \\
\hline & Jan-98 & Dec-02 & 60 & -0.24 & -3.73 & yes \\
\hline & Jan-99 & Dec-03 & 60 & -0.23 & -2.87 & yes \\
\hline & Jan-00 & Dec-04 & 60 & -0.05 & -1.26 & no \\
\hline & Jan-01 & Dec-05 & 60 & -0.10 & -2.23 & yes \\
\hline \multirow[t]{12}{*}{ Zambia } & Jan-90 & Dec-94 & 60 & -0.11 & -2.65 & yes \\
\hline & Jan-91 & Dec-95 & 60 & -0.09 & -2.65 & yes \\
\hline & Jan-92 & Dec-96 & 60 & -0.09 & -2.57 & yes \\
\hline & Jan-93 & Dec-97 & 60 & -0.08 & -3.01 & yes \\
\hline & Jan-94 & Dec-98 & 60 & -0.08 & -4.14 & yes \\
\hline & Jan-95 & Dec-99 & 60 & -0.04 & -1.48 & no \\
\hline & Jan-96 & Dec-00 & 60 & -0.03 & -0.96 & no \\
\hline & Jan-97 & Dec-01 & 60 & -0.11 & -2.72 & yes \\
\hline & Jan-98 & Dec-02 & 60 & -0.08 & -1.29 & no \\
\hline & Jan-99 & Dec-03 & 60 & -0.15 & -2.17 & yes \\
\hline & Jan-00 & Dec-04 & 60 & -0.06 & -1.36 & no \\
\hline & Jan-01 & Dec-05 & 60 & -0.06 & -1.35 & no \\
\hline \multicolumn{7}{|c|}{ Panel C: Cointegration of national deposit rate and national central bank policy rate } \\
\hline \multirow[t]{7}{*}{ Angola } & Jan-95 & Dec-99 & 60 & -0.07 & -1.11 & no \\
\hline & Jan-96 & Dec-00 & 60 & -0.02 & -0.52 & no \\
\hline & Jan-97 & Dec-01 & 60 & -0.51 & -4.22 & yes \\
\hline & Jan-98 & Dec-02 & 60 & -0.44 & -3.22 & yes \\
\hline & Jan-99 & Dec-03 & 60 & -0.20 & -1.92 & yes \\
\hline & Jan-00 & Dec-04 & 60 & -0.14 & -1.51 & no \\
\hline & Jan-01 & Dec-05 & 60 & -0.16 & -1.57 & no \\
\hline \multirow[t]{12}{*}{ Botswana } & Jan-90 & Dec-94 & 60 & -0.31 & -2.88 & yes \\
\hline & Jan-91 & Dec-95 & 60 & -0.13 & -1.61 & no \\
\hline & Jan-92 & Dec-96 & 60 & -0.18 & -2.14 & yes \\
\hline & Jan-93 & Dec-97 & 60 & -0.28 & -3.17 & yes \\
\hline & Jan-94 & Dec-98 & 60 & -0.59 & -5.34 & yes \\
\hline & Jan-95 & Dec-99 & 60 & -0.50 & -3.94 & yes \\
\hline & Jan-96 & Dec-00 & 60 & -0.11 & -2.11 & yes \\
\hline & Jan-97 & Dec-01 & 60 & -0.15 & -2.89 & yes \\
\hline & Jan-98 & Dec-02 & 60 & -0.27 & -4.10 & yes \\
\hline & Jan-99 & Dec-03 & 60 & -0.15 & -1.90 & yes \\
\hline & Jan-00 & Dec-04 & 60 & -0.24 & -2.52 & yes \\
\hline & Jan-01 & Dec-05 & 60 & -0.11 & -1.33 & no \\
\hline \multirow[t]{2}{*}{ Lesotho } & Jan-90 & Dec-94 & 60 & 0.00 & 0.04 & no \\
\hline & Jan-91 & Dec-95 & 60 & 0.02 & 0.26 & no \\
\hline
\end{tabular}


Table B-8: Kremers-Ericsson-Dolado cointegration analysis

\begin{tabular}{|c|c|c|c|c|c|c|}
\hline \multirow[t]{2}{*}{ Country } & \multicolumn{2}{|c|}{ Rolling sample } & \multirow[t]{2}{*}{ Obs } & \multicolumn{2}{|c|}{$\mathrm{ECT}_{\mathrm{t}-1}$} & \multirow[t]{2}{*}{ Coint? } \\
\hline & From & To & & Coeff & t-stat & \\
\hline \multirow[t]{10}{*}{ Lesotho } & Jan-92 & Dec-96 & 60 & -0.01 & -0.26 & $\overline{\text { no }}$ \\
\hline & Jan-93 & Dec-97 & 60 & -0.02 & -0.29 & no \\
\hline & Jan-94 & Dec-98 & 60 & -0.11 & -1.99 & yes \\
\hline & Jan-95 & Dec-99 & 60 & -0.09 & -1.43 & no \\
\hline & Jan-96 & Dec-00 & 60 & -0.04 & -0.93 & no \\
\hline & Jan-97 & Dec-01 & 60 & -0.02 & -0.72 & no \\
\hline & Jan-98 & Dec-02 & 60 & -0.06 & -1.28 & no \\
\hline & Jan-99 & Dec-03 & 60 & -0.31 & -4.69 & yes \\
\hline & Jan-00 & Dec-04 & 60 & -0.13 & -1.54 & no \\
\hline & Jan-01 & Dec-05 & 60 & -0.51 & -3.30 & yes \\
\hline \multirow[t]{11}{*}{ Madagascar } & Jan-91 & Dec-95 & 60 & -0.35 & -2.72 & yes \\
\hline & Jan-92 & Dec-96 & 60 & -0.51 & -4.05 & yes \\
\hline & Jan-93 & Dec-97 & 60 & -0.22 & -2.55 & yes \\
\hline & Jan-94 & Dec-98 & 60 & -0.14 & -2.06 & yes \\
\hline & Jan-95 & Dec-99 & 60 & -0.23 & -2.69 & yes \\
\hline & Jan-96 & Dec-00 & 60 & -0.24 & -3.28 & yes \\
\hline & Jan-97 & Dec-01 & 60 & -0.15 & -2.77 & yes \\
\hline & Jan-98 & Dec-02 & 60 & -0.09 & -2.46 & yes \\
\hline & Jan-99 & Dec-03 & 60 & -0.14 & -3.06 & yes \\
\hline & Jan-00 & Dec-04 & 60 & -0.37 & -5.55 & yes \\
\hline & Jan-01 & Dec-05 & 60 & -0.22 & -2.69 & yes \\
\hline \multirow[t]{12}{*}{ Malawi } & Jan-90 & Dec-94 & 60 & -0.39 & -2.53 & yes \\
\hline & Jan-91 & Dec-95 & 60 & -0.30 & -3.03 & yes \\
\hline & Jan-92 & Dec-96 & 60 & -0.15 & -1.64 & no \\
\hline & Jan-93 & Dec-97 & 60 & -0.04 & -0.81 & no \\
\hline & Jan-94 & Dec-98 & 60 & -0.09 & -1.50 & no \\
\hline & Jan-95 & Dec-99 & 60 & -0.24 & -2.23 & yes \\
\hline & Jan-96 & Dec-00 & 60 & -0.42 & -3.21 & yes \\
\hline & Jan-97 & Dec-01 & 60 & -0.27 & -3.00 & yes \\
\hline & Jan-98 & Dec-02 & 60 & -0.39 & -4.09 & yes \\
\hline & Jan-99 & Dec-03 & 60 & -0.31 & -3.05 & yes \\
\hline & Jan-00 & Dec-04 & 60 & -0.31 & -3.32 & yes \\
\hline & Jan-01 & Dec-05 & 60 & -0.47 & -5.00 & yes \\
\hline \multirow[t]{5}{*}{ Mauritius } & Jan-90 & Dec-94 & 60 & -0.29 & -2.44 & yes \\
\hline & Jan-91 & Dec-95 & 60 & -0.29 & -2.48 & yes \\
\hline & Jan-92 & Dec-96 & 60 & -0.23 & -2.10 & yes \\
\hline & Jan-93 & Dec-97 & 60 & -0.10 & -1.18 & no \\
\hline & Jan-94 & Dec-98 & 55 & -0.11 & -1.35 & no \\
\hline \multirow[t]{8}{*}{ Mozambique } & Jan-94 & Dec-98 & 60 & -0.17 & -2.19 & yes \\
\hline & Jan-95 & Dec-99 & 60 & -0.17 & -2.38 & yes \\
\hline & Jan-96 & Dec-00 & 60 & -0.15 & -2.44 & yes \\
\hline & Jan-97 & Dec-01 & 60 & -0.24 & -2.20 & yes \\
\hline & Jan-98 & Dec-02 & 60 & -0.01 & -0.65 & no \\
\hline & Jan-99 & Dec-03 & 60 & -0.02 & -1.20 & no \\
\hline & Jan-00 & Dec-04 & 60 & -0.02 & -0.99 & no \\
\hline & Jan-01 & Dec-05 & 60 & -0.01 & -0.34 & no \\
\hline \multirow[t]{11}{*}{ Namibia } & Jan-91 & Dec-95 & 52 & -0.53 & -4.98 & yes \\
\hline & Jan-92 & Dec-96 & 60 & -0.15 & -2.15 & yes \\
\hline & Jan-93 & Dec-97 & 60 & -0.27 & -4.86 & yes \\
\hline & Jan-94 & Dec-98 & 60 & -0.24 & -4.26 & yes \\
\hline & Jan-95 & Dec-99 & 60 & -0.20 & -3.62 & yes \\
\hline & Jan-96 & Dec-00 & 60 & -0.22 & -3.99 & yes \\
\hline & Jan-97 & Dec-01 & 60 & -0.24 & -4.35 & yes \\
\hline & Jan-98 & Dec-02 & 60 & -0.30 & -5.33 & yes \\
\hline & Jan-99 & Dec-03 & 60 & -0.20 & -4.21 & yes \\
\hline & Jan-00 & Dec-04 & 60 & -0.17 & -3.20 & yes \\
\hline & Jan-01 & Dec-05 & 60 & -0.20 & -3.72 & yes \\
\hline \multirow[t]{2}{*}{ South Africa } & Jan-90 & Dec-94 & 60 & -0.09 & -1.32 & no \\
\hline & Jan-91 & Dec-95 & 60 & -0.14 & -1.50 & no \\
\hline
\end{tabular}


Table B-8: Kremers-Ericsson-Dolado cointegration analysis

\begin{tabular}{|c|c|c|c|c|c|c|}
\hline \multirow[t]{2}{*}{ Country } & \multicolumn{2}{|c|}{ Rolling sample } & \multirow[t]{2}{*}{ Obs } & \multicolumn{2}{|c|}{$\mathrm{ECT}_{\mathrm{t}-1}$} & \multirow[t]{2}{*}{ Coint? } \\
\hline & From & To & & Coeff & t-stat & \\
\hline \multirow[t]{10}{*}{ South Africa } & Jan-92 & $\overline{\text { Dec-96 }}$ & 60 & -0.28 & -2.72 & yes \\
\hline & Jan-93 & Dec-97 & 60 & -0.43 & -3.31 & yes \\
\hline & Jan-94 & Dec-98 & 60 & -0.60 & -5.08 & yes \\
\hline & Jan-95 & Dec-99 & 60 & -0.41 & -3.68 & yes \\
\hline & Jan-96 & Dec-00 & 60 & -0.40 & -4.06 & yes \\
\hline & Jan-97 & Dec-01 & 60 & -0.49 & -4.64 & yes \\
\hline & Jan-98 & Dec-02 & 60 & -0.53 & -4.76 & yes \\
\hline & Jan-99 & Dec-03 & 60 & -0.26 & -3.30 & yes \\
\hline & Jan-00 & Dec-04 & 60 & -0.22 & -3.21 & yes \\
\hline & Jan-01 & Dec-05 & 60 & -0.34 & -3.60 & yes \\
\hline \multirow[t]{12}{*}{ Seychelles } & Jan-90 & Dec-94 & 60 & -0.10 & -1.19 & no \\
\hline & Jan-91 & Dec-95 & 60 & -0.09 & -1.34 & no \\
\hline & Jan-92 & Dec-96 & 60 & -0.16 & -2.16 & yes \\
\hline & Jan-93 & Dec-97 & 60 & -0.18 & -2.48 & yes \\
\hline & Jan-94 & Dec-98 & 60 & -0.11 & -1.90 & yes \\
\hline & Jan-95 & Dec-99 & 60 & -0.17 & -2.57 & yes \\
\hline & Jan-96 & Dec-00 & 60 & -0.30 & -3.85 & yes \\
\hline & Jan-97 & Dec-01 & 60 & -0.30 & -3.26 & yes \\
\hline & Jan-98 & Dec-02 & 60 & -0.36 & -3.75 & yes \\
\hline & Jan-99 & Dec-03 & 60 & -0.58 & -4.85 & yes \\
\hline & Jan-00 & Dec-04 & 60 & -0.34 & -2.65 & yes \\
\hline & Jan-01 & Dec-05 & 60 & -0.24 & -2.30 & yes \\
\hline \multirow[t]{12}{*}{ Swaziland } & Jan-90 & Dec-94 & 60 & -0.12 & -1.77 & yes \\
\hline & Jan-91 & Dec-95 & 60 & -0.01 & -0.25 & no \\
\hline & Jan-92 & Dec-96 & 60 & -0.04 & -1.59 & no \\
\hline & Jan-93 & Dec-97 & 60 & -0.16 & -2.76 & yes \\
\hline & Jan-94 & Dec-98 & 60 & -0.12 & -1.77 & yes \\
\hline & Jan-95 & Dec-99 & 60 & -0.57 & -5.21 & yes \\
\hline & Jan-96 & Dec-00 & 60 & -0.75 & -5.87 & yes \\
\hline & Jan-97 & Dec-01 & 60 & -0.30 & -2.90 & yes \\
\hline & Jan-98 & Dec-02 & 60 & -0.33 & -3.38 & yes \\
\hline & Jan-99 & Dec-03 & 60 & -0.16 & -2.14 & yes \\
\hline & Jan-00 & Dec-04 & 60 & -0.06 & -1.54 & no \\
\hline & Jan-01 & Dec-05 & 60 & -0.15 & -2.98 & yes \\
\hline \multirow[t]{8}{*}{ Tanzania } & Jan-94 & Dec-98 & 54 & -0.18 & -3.48 & yes \\
\hline & Jan-95 & Dec-99 & 60 & -0.22 & -4.39 & yes \\
\hline & Jan-96 & Dec-00 & 60 & -0.28 & -4.92 & yes \\
\hline & Jan-97 & Dec-01 & 60 & -0.35 & -5.76 & yes \\
\hline & Jan-98 & Dec-02 & 60 & -0.25 & -3.79 & yes \\
\hline & Jan-99 & Dec-03 & 60 & -0.18 & -3.63 & yes \\
\hline & Jan-00 & Dec-04 & 60 & -0.22 & -3.42 & yes \\
\hline & Jan-01 & Dec-05 & 60 & -0.21 & -2.36 & yes \\
\hline \multirow[t]{10}{*}{ Zambia } & Jan-92 & Dec-96 & 60 & -0.16 & -2.56 & yes \\
\hline & Jan-93 & Dec-97 & 60 & -0.18 & -3.06 & yes \\
\hline & Jan-94 & Dec-98 & 52 & -0.14 & -2.35 & yes \\
\hline & Jan-95 & Dec-99 & 41 & -0.21 & -3.22 & yes \\
\hline & Jan-96 & Dec-00 & 41 & -0.15 & -2.63 & yes \\
\hline & Jan-97 & Dec-01 & 41 & -0.13 & -2.32 & yes \\
\hline & Jan-98 & Dec-02 & 41 & -0.07 & -1.82 & yes \\
\hline & Jan-99 & Dec-03 & 49 & -0.13 & -2.28 & yes \\
\hline & Jan-00 & Dec-04 & 60 & -0.14 & -3.49 & yes \\
\hline & Jan-01 & Dec-05 & 60 & -0.12 & -3.39 & yes \\
\hline \multirow[t]{7}{*}{ Zimbabwe } & Jan-90 & Dec-94 & 60 & -0.19 & -2.62 & yes \\
\hline & Jan-91 & Dec-95 & 60 & -0.15 & -2.16 & yes \\
\hline & Jan-92 & Dec-96 & 60 & -0.05 & -0.87 & no \\
\hline & Jan-93 & Dec-97 & 60 & -0.11 & -2.04 & yes \\
\hline & Jan-94 & Dec-98 & 60 & -0.13 & -1.79 & yes \\
\hline & Jan-95 & Dec-99 & 60 & -0.13 & -2.11 & yes \\
\hline & Jan-96 & Dec-00 & 60 & -0.26 & -3.48 & yes \\
\hline
\end{tabular}


Table B-8: Kremers-Ericsson-Dolado cointegration analysis

\begin{tabular}{|c|c|c|c|c|c|c|}
\hline \multirow[t]{2}{*}{ Country } & \multicolumn{2}{|c|}{ Rolling sample } & \multirow[t]{2}{*}{ Obs } & \multicolumn{2}{|c|}{$\mathrm{ECT}_{\mathrm{t}-1}$} & \multirow[t]{2}{*}{ Coint? } \\
\hline & From & To & & Coeff & t-stat & \\
\hline \multirow[t]{5}{*}{ Zimbabwe } & Jan-97 & Dec-01 & 60 & -0.05 & -1.41 & no \\
\hline & Jan-98 & Dec-02 & 60 & -0.05 & -1.48 & no \\
\hline & Jan-99 & Dec-03 & 60 & -0.12 & -1.80 & yes \\
\hline & Jan-00 & Dec-04 & 60 & -0.71 & -4.83 & yes \\
\hline & Jan-01 & Dec-05 & 60 & -0.70 & -4.60 & yes \\
\hline \multicolumn{7}{|c|}{ Panel D: Cointegration of national deposit rate and South African central bank policy rate } \\
\hline \multirow[t]{12}{*}{$\overline{\text { Botswana }}$} & Jan-90 & Dec-94 & 60 & -0.07 & -1.14 & no \\
\hline & Jan-91 & Dec-95 & 60 & -0.13 & -1.60 & no \\
\hline & Jan-92 & Dec-96 & 60 & -0.08 & -0.99 & no \\
\hline & Jan-93 & Dec-97 & 60 & -0.14 & -1.59 & no \\
\hline & Jan-94 & Dec-98 & 60 & -0.80 & -5.28 & yes \\
\hline & Jan-95 & Dec-99 & 60 & -0.46 & -3.45 & yes \\
\hline & Jan-96 & Dec-00 & 60 & -0.08 & -1.44 & no \\
\hline & Jan-97 & Dec-01 & 60 & -0.09 & -1.44 & no \\
\hline & Jan-98 & Dec-02 & 60 & -0.04 & -0.72 & no \\
\hline & Jan-99 & Dec-03 & 60 & -0.09 & -1.76 & yes \\
\hline & Jan-00 & Dec-04 & 60 & -0.19 & -2.60 & yes \\
\hline & Jan-01 & Dec-05 & 60 & -0.28 & -2.69 & yes \\
\hline \multirow[t]{12}{*}{ Lesotho } & Jan-90 & Dec-94 & 60 & -0.26 & -2.62 & yes \\
\hline & Jan-91 & Dec-95 & 60 & -0.08 & -1.17 & no \\
\hline & Jan-92 & Dec-96 & 60 & -0.05 & -0.76 & no \\
\hline & Jan-93 & Dec-97 & 60 & -0.07 & -1.13 & no \\
\hline & Jan-94 & Dec-98 & 60 & -0.09 & -1.80 & yes \\
\hline & Jan-95 & Dec-99 & 60 & -0.04 & -0.83 & no \\
\hline & Jan-96 & Dec-00 & 60 & -0.08 & -1.74 & yes \\
\hline & Jan-97 & Dec-01 & 60 & -0.12 & -2.01 & yes \\
\hline & Jan-98 & Dec-02 & 60 & -0.29 & -3.63 & yes \\
\hline & Jan-99 & Dec-03 & 60 & -0.45 & -4.82 & yes \\
\hline & Jan-00 & Dec-04 & 60 & -0.36 & -3.31 & yes \\
\hline & Jan-01 & Dec-05 & 60 & -0.95 & -5.19 & yes \\
\hline \multirow[t]{8}{*}{ Mozambique } & Jan-94 & Dec-98 & 60 & -0.15 & -2.21 & yes \\
\hline & Jan-95 & Dec-99 & 60 & -0.09 & -1.83 & yes \\
\hline & Jan-96 & Dec-00 & 60 & -0.09 & -1.53 & no \\
\hline & Jan-97 & Dec-01 & 60 & -0.11 & -2.34 & yes \\
\hline & Jan-98 & Dec-02 & 60 & -0.04 & -1.59 & no \\
\hline & Jan-99 & Dec-03 & 60 & -0.03 & -1.55 & no \\
\hline & Jan-00 & Dec-04 & 60 & -0.02 & -0.75 & no \\
\hline & Jan-01 & Dec-05 & 60 & 0.03 & 0.92 & no \\
\hline \multirow[t]{11}{*}{ Namibia } & Jan-91 & Dec-95 & 60 & -0.60 & -6.15 & yes \\
\hline & Jan-92 & Dec-96 & 60 & -0.31 & -3.66 & yes \\
\hline & Jan-93 & Dec-97 & 60 & -0.36 & -4.20 & yes \\
\hline & Jan-94 & Dec-98 & 60 & -0.29 & -4.64 & yes \\
\hline & Jan-95 & Dec-99 & 60 & -0.21 & -3.43 & yes \\
\hline & Jan-96 & Dec-00 & 60 & -0.12 & -3.12 & yes \\
\hline & Jan-97 & Dec-01 & 60 & -0.19 & -4.30 & yes \\
\hline & Jan-98 & Dec-02 & 60 & -0.30 & -6.23 & yes \\
\hline & Jan-99 & Dec-03 & 60 & -0.21 & -4.71 & yes \\
\hline & Jan-00 & Dec-04 & 60 & -0.17 & -3.37 & yes \\
\hline & Jan-01 & Dec-05 & 60 & -0.20 & -3.76 & yes \\
\hline \multirow[t]{10}{*}{ Swaziland } & Jan-90 & Dec-94 & 60 & -0.06 & -1.63 & no \\
\hline & Jan-91 & Dec-95 & 60 & -0.24 & -3.60 & yes \\
\hline & Jan-92 & Dec-96 & 60 & -0.46 & -5.10 & yes \\
\hline & Jan-93 & Dec-97 & 60 & -0.68 & -7.15 & yes \\
\hline & Jan-94 & Dec-98 & 60 & -0.26 & -4.12 & yes \\
\hline & Jan-95 & Dec-99 & 60 & -0.30 & -4.04 & yes \\
\hline & Jan-96 & Dec-00 & 60 & -0.21 & -3.47 & yes \\
\hline & Jan-97 & Dec-01 & 60 & -0.23 & -4.06 & yes \\
\hline & Jan-98 & Dec-02 & 60 & -0.24 & -3.68 & yes \\
\hline & Jan-99 & Dec-03 & 60 & -0.17 & -2.51 & yes \\
\hline
\end{tabular}


Table B-8: Kremers-Ericsson-Dolado cointegration analysis

\begin{tabular}{lcccccc}
\hline Country & \multicolumn{2}{c}{ Rolling sample } & Obs & \multicolumn{2}{c}{ ECT $_{\text {t-1 }}$} & Coint? \\
\cline { 2 - 3 } \cline { 5 - 6 } Swaziland & From & To & & Coeff & t-stat & \\
& Jan-00 & Dec-04 & 60 & -0.03 & -1.22 & no \\
& Jan-01 & Dec-05 & 60 & -0.09 & -2.74 & yes \\
& Jan-90 & Dec-94 & 60 & -0.06 & -1.36 & no \\
& Jan-91 & Dec-95 & 60 & -0.06 & -1.75 & yes \\
& Jan-92 & Dec-96 & 60 & -0.06 & -1.81 & yes \\
& Jan-93 & Dec-97 & 60 & -0.06 & -2.27 & yes \\
& Jan-94 & Dec-98 & 60 & -0.07 & -2.33 & yes \\
& Jan-95 & Dec-99 & 60 & -0.04 & -1.35 & no \\
& Jan-96 & Dec-00 & 60 & -0.03 & -1.07 & no \\
& Jan-97 & Dec-01 & 60 & -0.11 & -3.10 & yes \\
& Jan-98 & Dec-02 & 60 & -0.02 & -0.53 & no \\
& Jan-99 & Dec-03 & 60 & -0.13 & -2.45 & yes \\
& Jan-00 & Dec-04 & 60 & -0.06 & -2.05 & yes \\
& Jan-01 & Dec-05 & 60 & -0.06 & -2.12 & yes \\
\hline
\end{tabular}

Note: Following Kremers, Ericsson and Dolado (1992), we consider cointegration to exist when the coefficient of the lagged error correction term $\left(\mathrm{ECT}_{\mathrm{t}-1}\right)$ is signfiicant in symmetric pass-though model. Significance is based on the t-statistic of the coefficient at $5 \%$ level. For 60 observations, this $t$ test has a critical value of 1.671. 